\title{
A ROBOT DESIGNED FOR WALKING AND CLIMBING BASED ON ABSTRACTED COCKROACH LOCOMOTION MECHANISMS
}

\author{
by \\ TERENCE WEI \\ Submitted in partial fulfillment of the requirements \\ For the degree of Doctor of Philosophy \\ Thesis Advisor: Professor Roger D. Quinn \\ Department of Mechanical and Aerospace Engineering \\ CASE WESTERN RESERVE UNIVERSITY
}

January 2006 


\section{CASE WESTERN RESERVE UNIVERSITY \\ SCHOOL OF GRADUATE STUDIES}

We hereby approve the dissertation of

Terence Wei

candidate for the Ph.D. degree *.

(signed) $\frac{\text { Roger D. Quinn }}{\text { (chair of the committee) }}$
Dwight T. Davy

Roy E. Ritzmann

Vikas Prakash

(date) $10 / 18 / 05$

*We also certify that written approval has been obtained for any proprietary material contained therein. 


\section{Table of Contents}

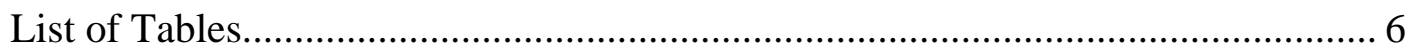

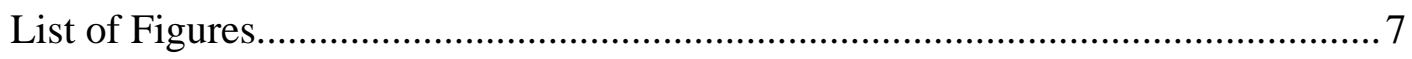

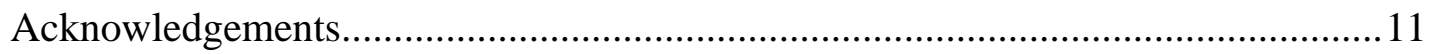

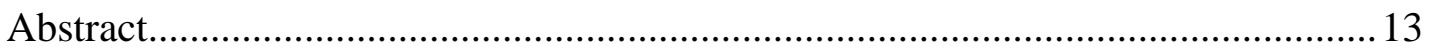

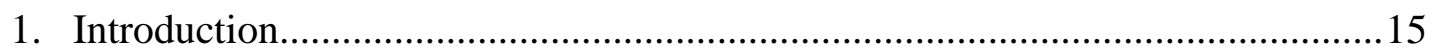

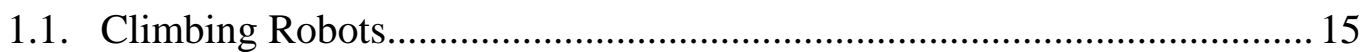

1.2. Abstracted Cockroach Locomotion Mechanisms.........................................16

1.3. Sagittal and Coronal Planes....................................................................... 17

1.4. MechaRoach II.......................................................................................19

1.5. Other Climbing Robots............................................................................ 21

2. Cockroach Treadmill................................................................................... 23

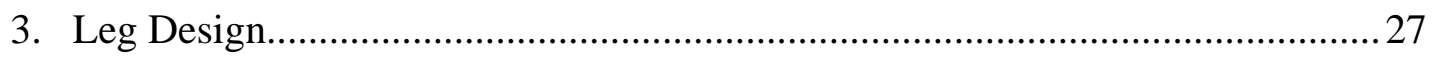

3.1. Key Features of Cockroach Foot Trajectories............................................. 27

3.2. Previous Approaches in Robotics............................................................ 29

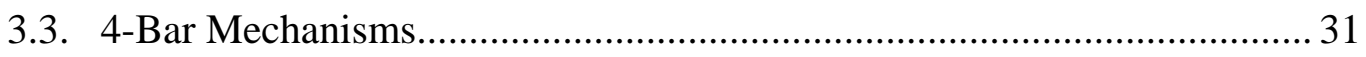

3.4. Mechanism Design Goals.......................................................................... 33

3.5. The Mechanism Design Method.......................................................................

3.5.1. Graphical Synthesis...................................................................... 37

3.5.2. Working Model 2D......................................................................... 40

3.5.3. Lego Prototyping............................................................................. 42

3.5.4. The Mechanism Analyzer Program...................................................... 44

3.5.5. Evaluation of Finished Legs.............................................................. 56 
3.6. Poor Mechanism Design Example.

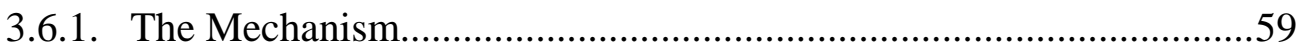

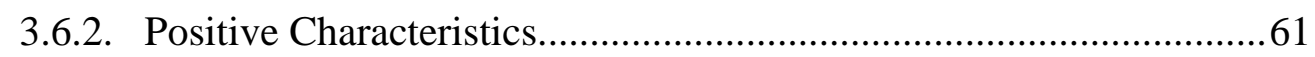

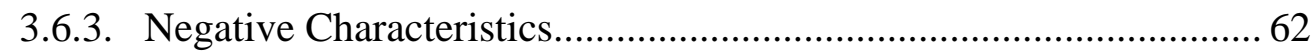

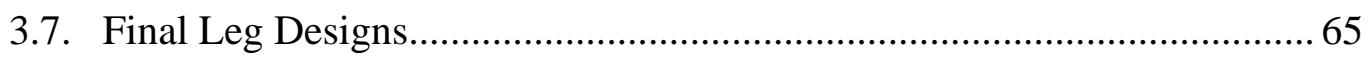

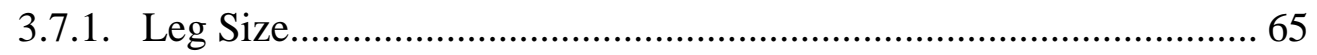

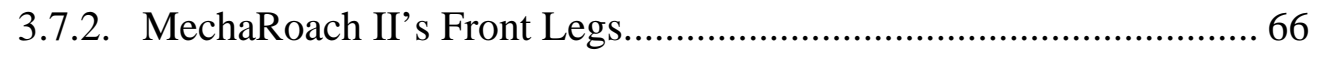

3.7.3. MechaRoach II's Middle Legs............................................................. 71

3.7.4. MechaRoach II's Rear Legs.............................................................. 74

3.7.5. Mechanism Construction................................................................. 78

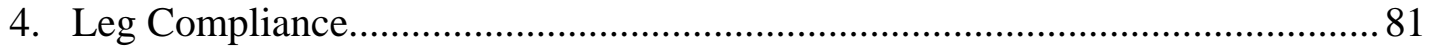

4.1. The Need for Leg Compliance................................................................... 81

4.2. Leg Compliance on MechaRoach II............................................................ 84

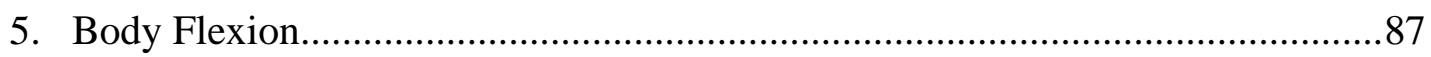

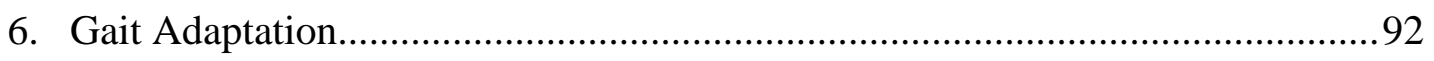

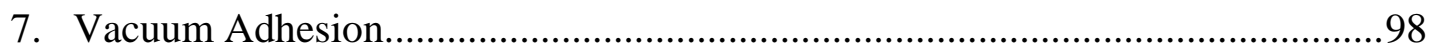

7.1. Vortex Regenerated Air Movement...........................................................98

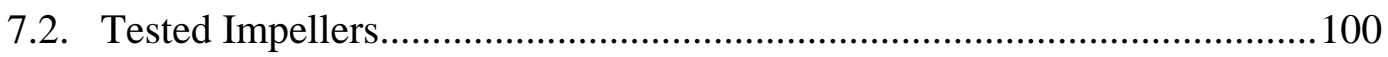

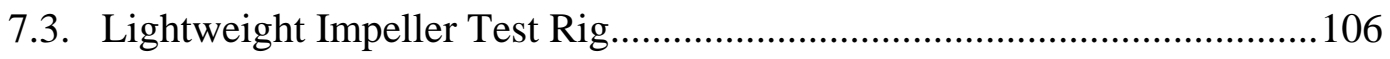

7.4. Instrumented Impeller Test Rig.................................................................. 108

7.4.1. Test Rig Construction........................................................................ 108

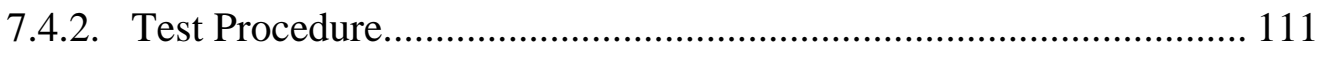

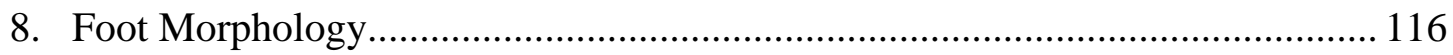




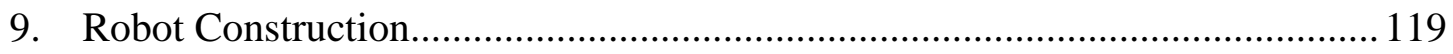

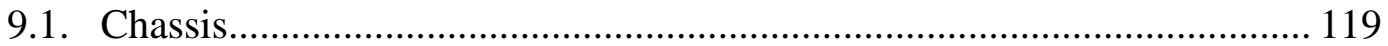

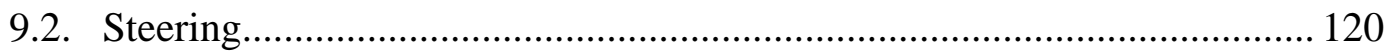

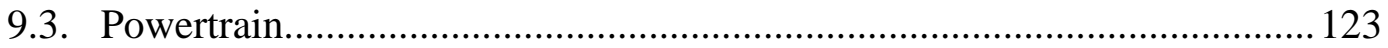

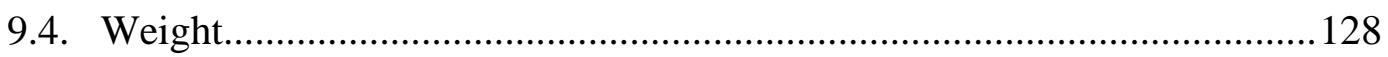

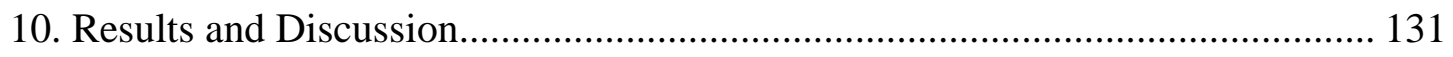

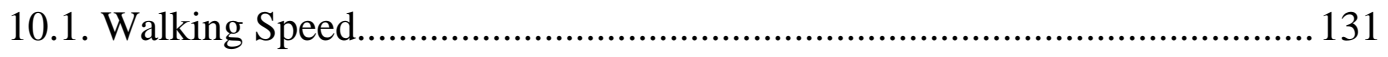

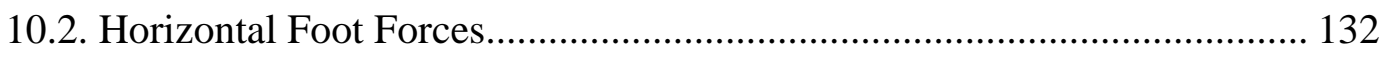

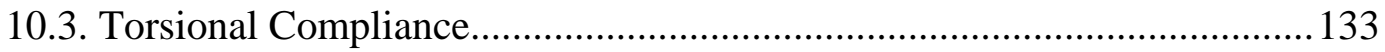

10.4. Leg Coordination and Turning.............................................................141

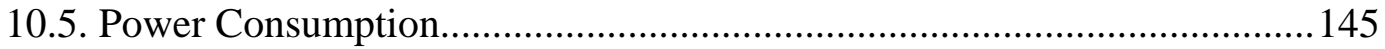

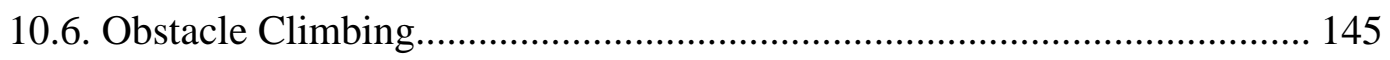

10.7. Transitioning Between Horizontal and Inclined Surfaces........................ 148

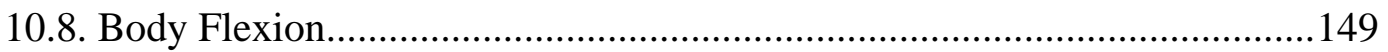

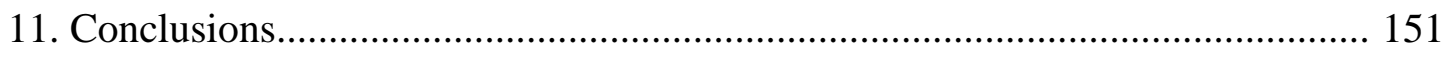

Appendix A. The Mechanism Analyzer Program................................................. 159

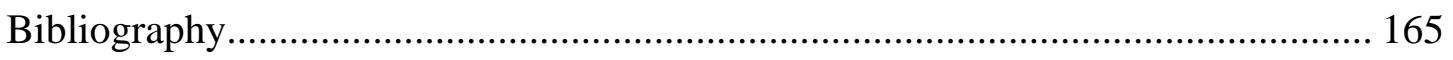




\section{List of Tables}

Table 7-1. Physical Descriptions of the Thirteen Tested Impellers..............................106

Table 7-2. Generated Adhesion Forces by Tested Impellers......................................113 


\section{List of Figures}

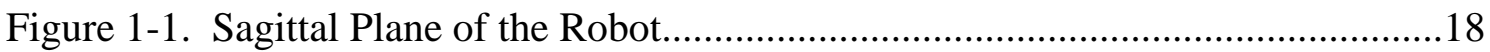

Figure 1-2. Coronal Plane of the Robot......................................................................18

Figure 1-3. MechaRoach II......................................................................................... 19

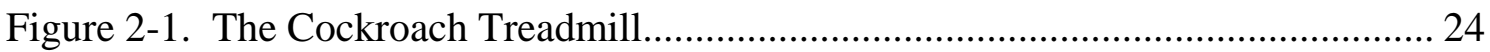

Figure 3-1. Cockroach Front Leg Raised High During Swing

(Courtesy of Ritzmann Lab).......................................................... 27

Figure 3-2. Cockroach Footpaths During Walking [7]...............................................28

Figure 3-3. A Wheeled Vehicle Moving at a High Rate of Speed.................................. 29

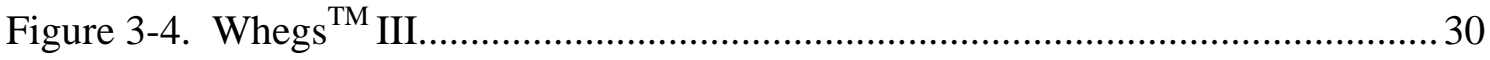

Figure 3-5. A Front 4-Bar Mechanism Leg.................................................................. 32

Figure 3-6. The Relationship Between CG Height and

Necessary Attachment Force.................................................................. 36

Figure 3-7. Three-Position Graphical Synthesis................................................................39

Figure 3-8. Lego Prototype of Front Leg......................................................................43

Figure 3-9. Diagramed Front Leg Mechanism................................................................ 46

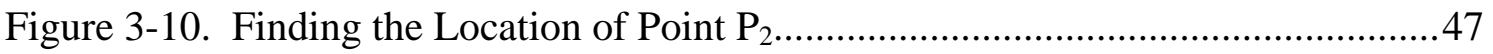

Figure 3-11. Finding Instant Centers.......................................................................... 48

Figure 3-12. Finding the Tibia’s Instant Center..............................................................49

Figure 3-13. The Crank and Coupler are like Two Gears in Mesh..................................50

Figure 3-14. Instant Center Locations Move Discontinuously........................................50

Figure 3-15. Front Leg Instant Center Positions.............................................................51

Figure 3-16. Zoomed View of Front Leg Instant Center Positions................................. 52 
Figure 3-17. Tibia Movement Radius vs. Crank Position......................................... 53

Figure 3-18. Pathological Case for Mechanical Advantage Calculations...................... 54

Figure 3-19. Parts and Assemblies Created in Pro/ENGINEER................................. 56

Figure 3-20. A Small, Scaled Prototype of the Front Leg........................................... 57

Figure 3-21. Poor 4-Bar Mechanism Leg Example..................................................59

Figure 3-22. Example Mechanism Footpath.......................................................60

Figure 3-23. Example Mechanism Foot Velocity Components..................................62

Figure 3-24. Example Mechanism Mechanical Advantage.........................................63

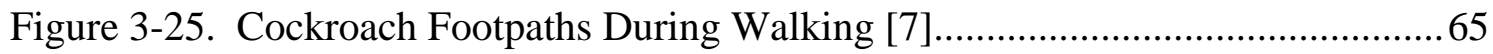

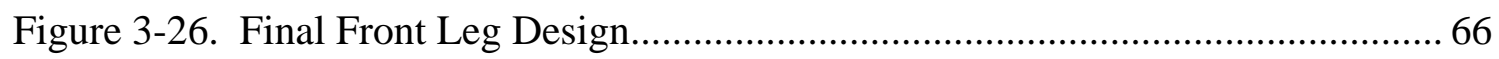

Figure 3-27. MechaRoach II’s Front Leg Footpath..............................................67

Figure 3-28. Crank Angle Definition..............................................................68

Figure 3-29. Front Foot Velocity Components vs. Crank Position.............................. 69

Figure 3-30. Front Leg Mechanical Advantage vs. Crank Position............................ 70

Figure 3-31. Final Middle Leg Design............................................................. 71

Figure 3-32. MechaRoach II’s Middle Leg Footpath.............................................. 72

Figure 3-33. Middle Foot Velocity Components vs. Crank Position........................... 73

Figure 3-34. Middle Leg Mechanical Advantage vs. Crank Position........................... 74

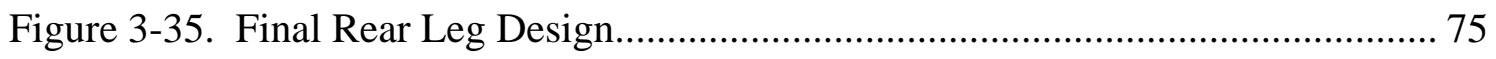

Figure 3-36. MechaRoach II’s Rear Leg Footpath.................................................. 75

Figure 3-37. Rear Foot Velocity Components vs. Crank Position............................... 77

Figure 3-38. Rear Leg Mechanical Advantage vs. Crank Position..............................78

Figure 3-39. Crank and Rocker Each Consist of Two Identical Pieces.........................79 


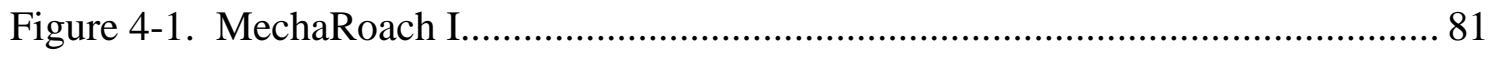

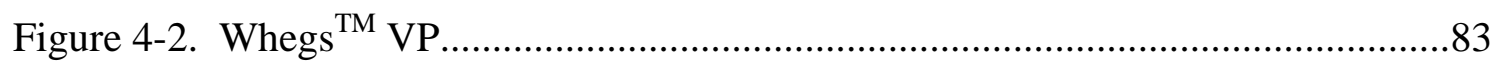

Figure 4-3. Front Leg Compliance Components................................................... 85

Figure 4-4. Leg Compliance in Unloaded (left) and Loaded (right) States....................85

Figure 5-1. Cockroach Body Flexion Joint (Courtesy of Ritzmann Lab).....................87

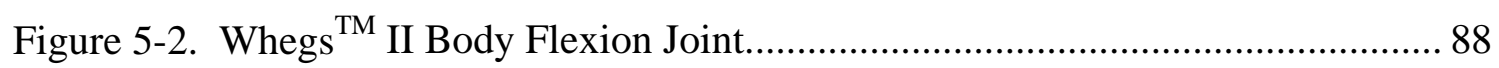

Figure 5-3. Side View of Robot and Body Flexion Joint......................................... 89

Figure 5-4. Body Flexion Joint Flexed and Unflexed...............................................90

Figure 6-1. Gait Adaptation with Whegs ${ }^{\mathrm{TM}}$.......................................................... 92

Figure 6-2. Passive Torsionally Compliant Device................................................. 93

Figure 6-3. Exploded View of Passive Torsionally Compliant Device.........................93

Figure 6-4. MechaRoach I Gait Adaptation..........................................................95

Figure 6-5. Middle Leg Footpath Trajectory........................................................96

Figure 7-1. Theoretical Calculation of Adhesion Force............................................ 99

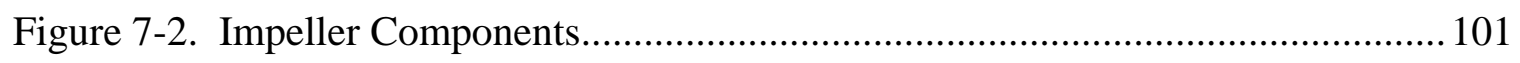

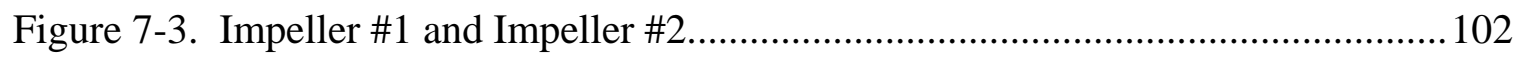

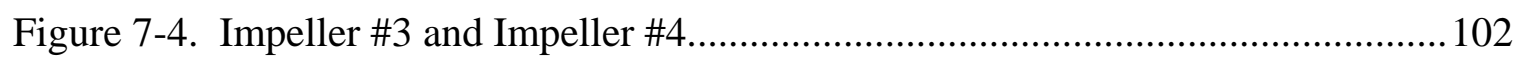

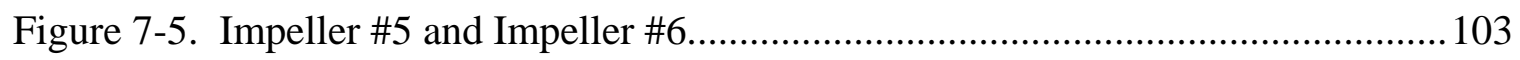

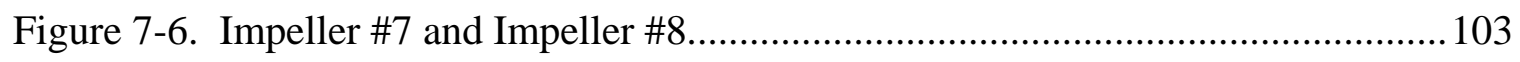

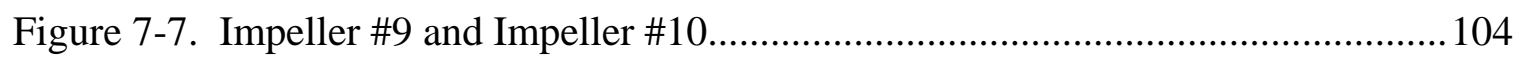

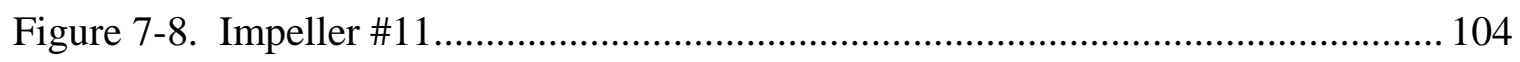

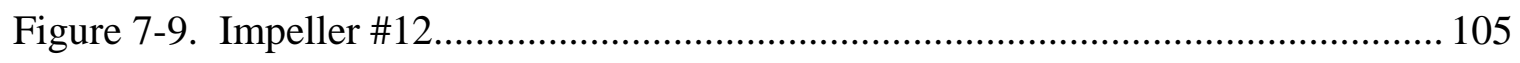

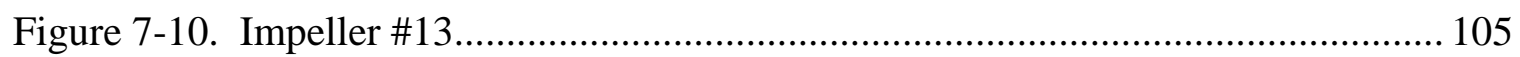


Figure 7-11. Lightweight Impeller Test Rig........................................................ 107

Figure 7-12. Instrumented Impeller Test Rig....................................................... 109

Figure 7-13. Plexiglas Plate From Underneath................................................ 110

Figure 7-14. Adhesion Force Generated by Impellers.............................................113

Figure 7-15. Voltage Required to Spin Impellers.................................................. 115

Figure 8-1. Cockroach Tarsus (Courtesy of Sasha Zill)............................................ 116

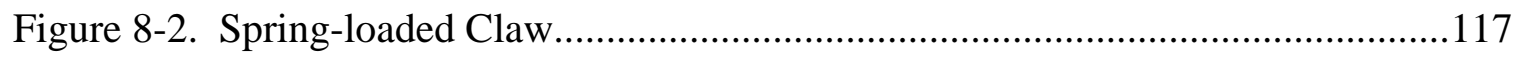

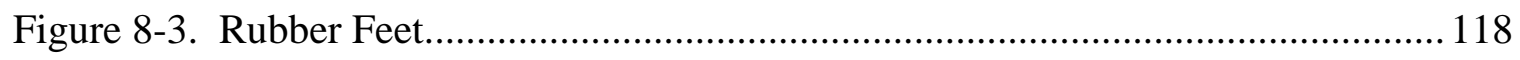

Figure 9-1. Bare Chassis of MechaRoach II..........................................................119

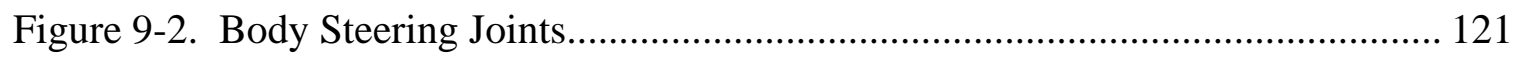

Figure 9-3. Transverse-mounted Steering Motor.......................................................122

Figure 9-4. Drivechains Cause Changes in Leg Coordination.................................... 125

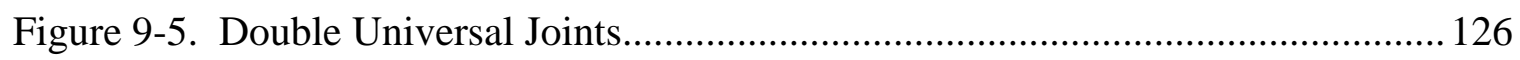

Figure 9-6. Double Universal Joints Cause Driveshaft Longitudinal Movement.......... 127

Figure 9-7. Ball-spline in Rear Section of Robot................................................... 128

Figure 9-8. Distribution of Mass about the Robot................................................... 130

Figure 10-1. Percentage of Front Leg Time Spent in Stance.....................................136

Figure 10-2. Percentage of Middle Leg Time Spent in Stance....................................138

Figure 10-3. Percentage of Rear Leg Time Spent in Stance.......................................139

Figure 10-4. Gait Diagram for MechaRoach II with No Torsional Compliance.............142

Figure 10-5. Gait Diagram for MechaRoach II with Torsional Compliance..................143

Figure 10-4. Transitioning without Use of Body Flexion Joint...................................150

Figure 10-5. Transitioning with Use of Body Flexion Joint......................................150 
Figure A-1. Finding the Intersection of Two Circles.................................................164 


\section{Acknowledgements}

Completing a dissertation is a long road to follow, and it has been challenging far beyond what I could have expected. Along the way, I have learned an amazing amount about science, engineering, politics, academics, and a lot of things that were only tangentially related to the research. None of this would have been possible without the support and patience of colleagues, friends and loved ones.

In particular, I would like to thank my advisor Roger Quinn, for giving me all of the opportunities I have had throughout my entire post-graduate academic career. He has been generous with advice and guidance, while giving me huge amounts of latitude in developing projects. He has not only created a workplace where lots of interesting projects can happen, but one in which everyone has fun while doing it.

Roy Ritzmann and the lab that he directs have been collaborating with the Biologically-Inspired Robotics Laboratory for some time now. Not only has the collaboration been extremely educational, but also it has caused me to be interested in areas of science that I never knew I would be interested in. His unique views have made this project more well-rounded and better thought-out.

I'd also like to thank the rest of my dissertation committee, Dwight Davy and Vikas Prakash for keeping me on track, for their guidance and suggestions, and for the time they have devoted to this project.

The work presented in this document was funded by US Air Force contracts F0863001-C-0023 and F08630-03-1-0003, and the National Science Foundation through the NSF/IGERT Neuromechanics grant DGE 9972747. Without their involvement, it would not have been possible to conduct this research. 
The personnel of the Biologically-Inspired Robotics Laboratory have given me so much, and have been so openhanded with their time. They have allowed me to constantly bounce ideas off of them, introduced me to new techniques and methods I had not known previously, and devoted countless hours to helping me proofread documents and presentations. In particular, I would like to acknowledge Matthew Birch and Andrew Horchler for lending their considerable design expertise, and William Lewinger for his expertise with electronics. Most importantly, the members of the lab have participated in ludicrous amounts of goofing off over the years, like creating the game of Death Frisbee, which has kept me sane. Their friendship has made the experience more meaningful and enjoyable.

I would like to thank my parents, who encouraged me to go down this road in the first place, and for being so generous and supportive my entire life. They always made sure I pushed myself to achieve more. They have made it possible for me to even have the opportunities to go to Case and graduate school in the first place.

Last, but not least, I would like to thank my wife for being incredibly patient, supportive and understanding. She continued this even after witnessing, literally thousands of times, people asking me when I was going to graduate. 
A Climbing and Walking Robot Based on

Abstracted Cockroach Locomotion Mechanisms

Abstract

by

\section{TERENCE WEI}

MechaRoach II is a hexapod robot that can walk on horizontal and inclined surfaces, and was designed to test strategies for transitioning between the two. The locomotion principles that allow cockroaches to make these transitions have been studied and mechanisms using abstractions of those principles have been developed for the robot. These principles included usage of features of leg morphology, leg compliance, gait adaptation, and body flexion. MechaRoach II has a single drive motor, a motor for steering, and a motor to actuate a body flexion joint. The single drive motor powers all six legs, and each leg uses 4-bar mechanisms to recreate cockroach-like foot trajectories. Cockroaches have been shown to flex their bodies downward between the first and second thoracic segments or rear their bodies upward using their middle legs during transitions between climbing on vertical surfaces and walking on horizontal ones. Similarly, MechaRoach II’s body joint rears the front of the robot upward or downward during transitioning. The robot normally walks in a tripod gait with contralateral legs 180 degrees out of phase, but uses passive torsionally compliant devices to bring contralateral legs into phase for climbing. The robot measures $66 \mathrm{~cm}$ long, $40 \mathrm{~cm}$ wide, 
and $10 \mathrm{~cm}$ tall. At $7 \mathrm{~kg}$, MechaRoach II has a weight similar to a cockroach scaled up to its size. Although power-autonomous, the robot requires a human operator communicating to it by way of radio-control. 


\section{Introduction}

\subsection{Climbing Robots}

Robots with the ability to climb inclined or vertical surfaces have numerous applications. Legged robots have already been developed to climb on the underside of bridges [1] and crawl on the insides of pipes [13] for inspection purposes. Other climbing robots have been developed for jobs such as weld inspection in nuclear plants [45]. Robots are the ideal choice for many of these applications because the working environments can be either poor or hazardous for humans.

In the process of completing one of these tasks, a climbing robot may have to negotiate a transition between surfaces that are at different inclinations, such as between a horizontal surface and a vertical one. Successful negotiation of that transition will require more than just the ability to adhere to the surfaces. MechaRoach II was designed and built to test ideas for how these transitions can be made.

There are only a few legged robots that can walk on horizontal surfaces, climb vertical surfaces, and make the transition between the two. They include Robug IIs [23], Robug III [24], Ninja-1 [16], and Ninja-II [15]. These robots take advantage of legs that allow for larger ranges of motion than a normal walking robot would have. They also tend to be very large, use lots of actuators, and are complex to control.

The purpose of this work was to develop a robot to test strategies that robots could use to make the transition between walking on horizontal surfaces and climbing on inclined surfaces. 


\subsection{Abstracted Cockroach Locomotion Mechanisms}

Cockroaches are ideal for modeling robots after, because they are able to move quickly and scramble over obstacles that are relatively tall compared to their own size. A robot that could move as quickly as a scaled up cockroach, or could climb over obstacles like cockroaches would have plenty of practical applications. In order to give the robot cockroach-like mobility, the methods and techniques that cockroaches use during locomotion have been studied and have been attempted to be reproduced.

Cockroaches have been shown to employ many different locomotion principles when they switch between walking on a horizontal surface and climbing on a vertical or inclined one, such as usage of features of leg morphology, leg compliance, gait adaptation, and body flexion. It also includes usage of foot morphology such as pads on their tarsi that allow them to grip a variety of surfaces [18] and actuated claws [11]. Mechanical devices that employ these principles can be used to allow robots to successfully negotiate transitions between surfaces of different inclinations.

Exactly reproducing the behavior of a biological system may be time consuming, overly complex and require the development of new technologies. In addition, biological systems may have capabilities that are not relevant to locomotion. For instance, joint ranges of motion may allow for feeding or reproduction but may not be used during locomotion. Abstracting the useful biological principles by implementing them in simpler mechanical devices can more quickly result in mission-capable robots [3]. Other robots that use abstracted biological locomotion principles include PROLERO [25], RHex [38], Whegs ${ }^{\mathrm{TM}}$ [3], and MechaRoach I [7]. The design of MechaRoach II benefits from abstracted biological principles that cockroaches use to walk and climb. 
Four abstracted cockroach locomotion principles have been implemented into MechaRoach II. They include features of leg morphology, leg compliance, body flexion, and gait adaptation.

\subsection{Sagittal and Coronal Planes}

In explanations of various features of the robot and cockroach, frequent references will be made to things that occur in the horizontal and vertical planes, with respect to the robot's chassis or animal's body. Since the robot was designed for climbing and operated on surfaces that are not horizontal, references to the horizontal and vertical planes would be confusing. Furthermore, even if the robot always operated on a horizontal surface, references to a vertical plane may still be ambiguous.

Therefore, all of the explanations will refer to the sagittal and coronal planes of the robot or animal. The sagittal plane will refer to a plane that runs along the midline, which divides the chassis or body into left and right halves (see Figure 1-1). 


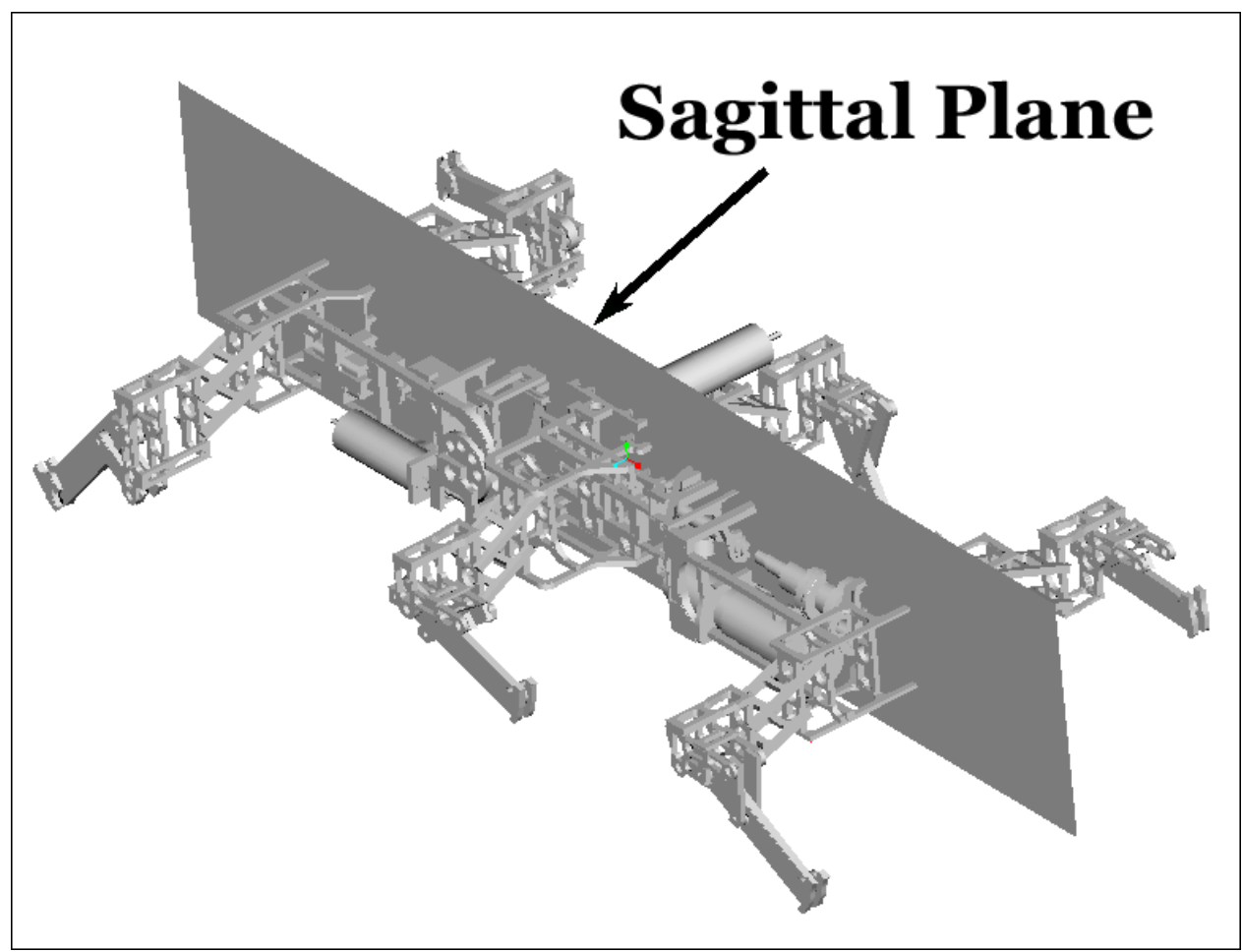

Figure 1-1. Sagittal Plane of the Robot

The coronal plane, in contrast, divides the chassis or body into dorsal and ventral halves (see Figure 1-2).

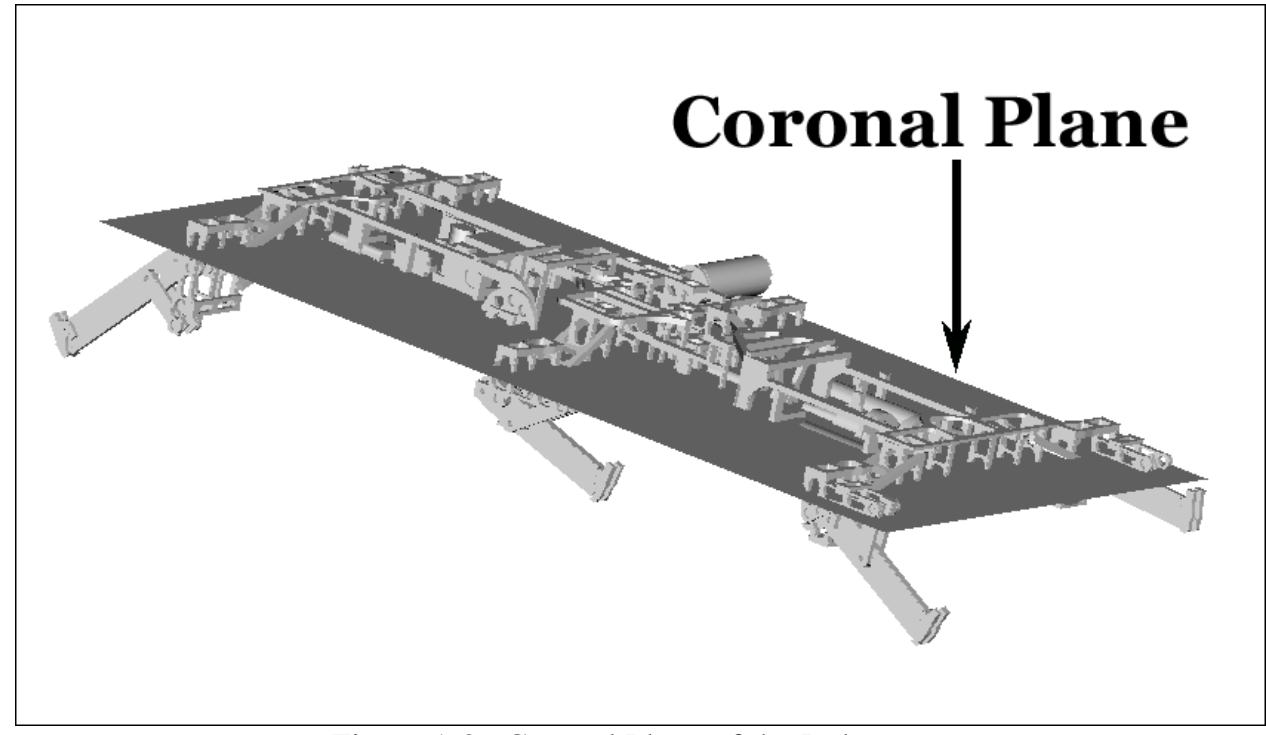

Figure 1-2. Coronal Plane of the Robot 


\subsection{MechaRoach II}

MechaRoach II is shown in Figure 1-3. It has six legs, measures $66 \mathrm{~cm}$ long, $40 \mathrm{~cm}$ wide, and $10 \mathrm{~cm}$ tall and weighs $7 \mathrm{~kg}$. These dimensions made the robot a convenient size to manufacture given the available in-house equipment, although the concept could be scaled up or down in size. The frame is made of a network of thin aluminum supports, which allows it to be relatively lightweight. The body length is roughly 15 times longer than a typical Blaberus discoidalis cockroach. A typical cockroach mass is $2.25 \mathrm{~g}$ [21]. If the animal were scaled up 15 times in length, its mass would increase by length cubed according to dynamic scaling and it would weigh about $7.59 \mathrm{~kg}$. The robot, therefore, has a proportional mass compared to the cockroach.

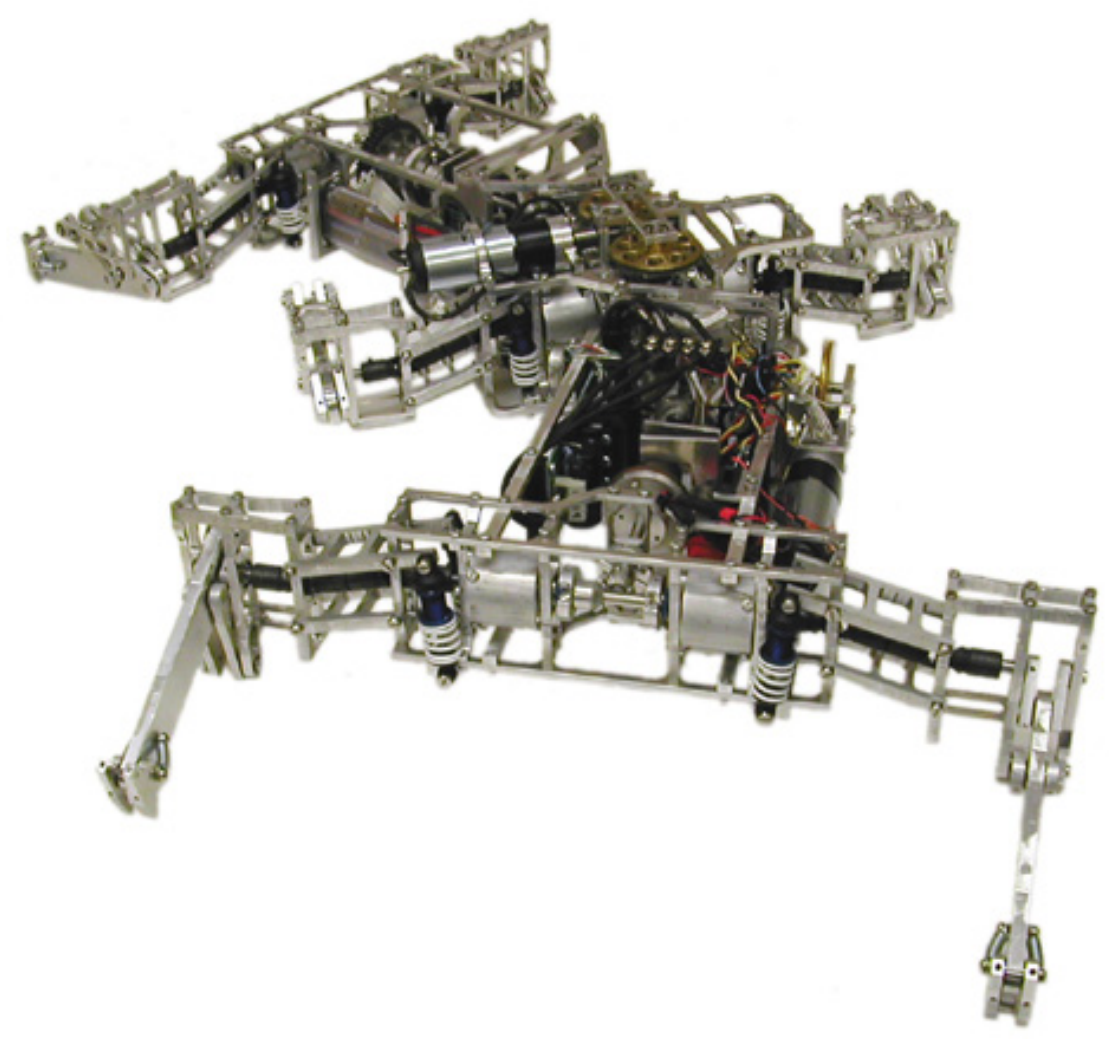

Figure 1-3. MechaRoach II 
MechaRoach II has three motors: A 90-Watt motor for propulsion, a 20-Watt motor for steering and a 20-Watt motor for vertical body flexion. The use of a single drive motor allows a higher power-to-weight ratio to be attainable compared to having a motor for each leg [3]. When climbing over an obstacle, the robot may find itself in an orientation where only one leg has a foothold. If each leg had a separate motor, each of those motors would have to generate enough torque to propel the robot forward in that situation. Instead, the robot has a single drive motor and all of the motor torque can be delivered to any of the legs. As a result, the robot needs only a single powerful motor, instead of six. Since motors are usually the most massive components on a robot, it is easy to see how having only one drive motor results in a higher power-to-weight ratio.

For example, RHex has a single drive motor per leg [38]. Whegs II showed that having a single, powerful drive motor that allows all of the motor torque to be delivered to any of its legs would have a superior power-to-weight ratio $\left(103 \mathrm{~W} \mathrm{~kg}^{-1}\right)$ compared to $67 \mathrm{~W} \mathrm{~kg}^{-1}$ for RHex [3, 33].

Although power-autonomous, MechaRoach II requires a human operator communicating with it by way of radio-control. Power for the motors comes from a single 7.2-volt, 3000mAh rechargeable battery, and power for the radio-control receiver is provided by a single 4.8 -volt, $600 \mathrm{mAh}$ rechargeable battery.

To turn, the robot has body joints that allow the front and rear segments of the robot to pivot left and right independently of the middle segment, as shown in Figure 1-1. This motion is independent of the vertical body flexion, which permits the front half of the robot to rotate up and down in the sagittal plane, independently of the middle and rear 
sections of the robot. This range of motion was designed to ease the vehicle's transition to and from horizontal and inclined surfaces.

The leg morphology and gait adaptation have been implemented in a way that embedded the locomotion controller into the mechanics, which simplified the control problem. Each of the legs consists of a 4-bar mechanism that recreates cockroach-like foot trajectories when viewed in the sagittal plane. Passive torsionally compliant devices mounted in series with the robot's driveshafts allow MechaRoach II to walk in an alternating tripod gait, but bring leg pairs into phase to work together when climbing. Previous robots that had their locomotion controller embedded into their mechanics include Boadicea [6], which imitated cockroach footpaths, McGeer's passive-dynamic walkers [26], and the passive-dynamic and powered walking robots that have been developed by Ruina and Wisse [8,9].

The design of the legs allows other mechanisms or devices to be mounted onto the end of them. Therefore, it would be possible to mount magnets, suction cups or other types of attachment devices onto the ends of the legs.

\subsection{Previous Climbing Robots}

Climbing robots have utilized many types of attachment devices in order to climb vertical or inclined surfaces, including magnets [14], suction cups [22, 48], and miniature spines that catch surface asperities [20]. Other robots, instead of using end-effectors to create the attachment forces, have used suction to create a low-pressure area underneath the entire robot [27] and propellers to push the robot towards the substrate [28]. 
Usage of magnets or suction cups would have limited the robot to being operated in too few environments. One technique used for generating a low-pressure area underneath the robot was investigated for use on this robot. Generating a low-pressure area underneath the robot would have allowed operation on any flat surface, giving the robot a wider variety of surfaces it was capable of walking on.

Mechanical claws were also investigated for use on the robot. However, there are many other ways that a climbing robot could create attachment forces. One alternative to claws would be to use sticky pads on the feet of a robot to create adhesion to the substrate. This method would be analogous to the pads that cockroaches have on their tarsi [18], and the way that geckos use Van der Waals forces to adhere to surfaces [4,5]. Synthetic gecko foot-hairs have already been developed for use on climbing robots [42]. Furthermore, there are several robots that already use sticky pads for adhesion and peel back these pads from the substrate in a similar way that the gecko does, such as MechoGecko [40], Bull-Gecko [40], and Climbing Mini-Whegs ${ }^{\mathrm{TM}}$ [10]. 


\section{Cockroach Treadmill}

The research that made MechaRoach II and other robots possible was a result of collaboration between the Biologically Inspired Robotics Lab and the Insect Locomotion Lab at Case Western Reserve University. The Biologically Inspired Robotics Lab is directed by Professor R.D. Quinn and is located in the Mechanical and Aerospace Engineering department. The Insect Locomotion Lab is directed by Professor R.E. Ritzmann and is located in the Biology department.

A motorized treadmill was necessary to investigate the behavior of cockroaches as they made the transition between walking on horizontal and inclined surfaces, so that the methods they used could be implemented in a robot. The treadmill that was designed and built consisted of two major sections - a horizontal section and an inclined one (see Figure 2-1). The inclined section could rotate a total of 135 degrees, and at one extreme end of the range would be vertical. 


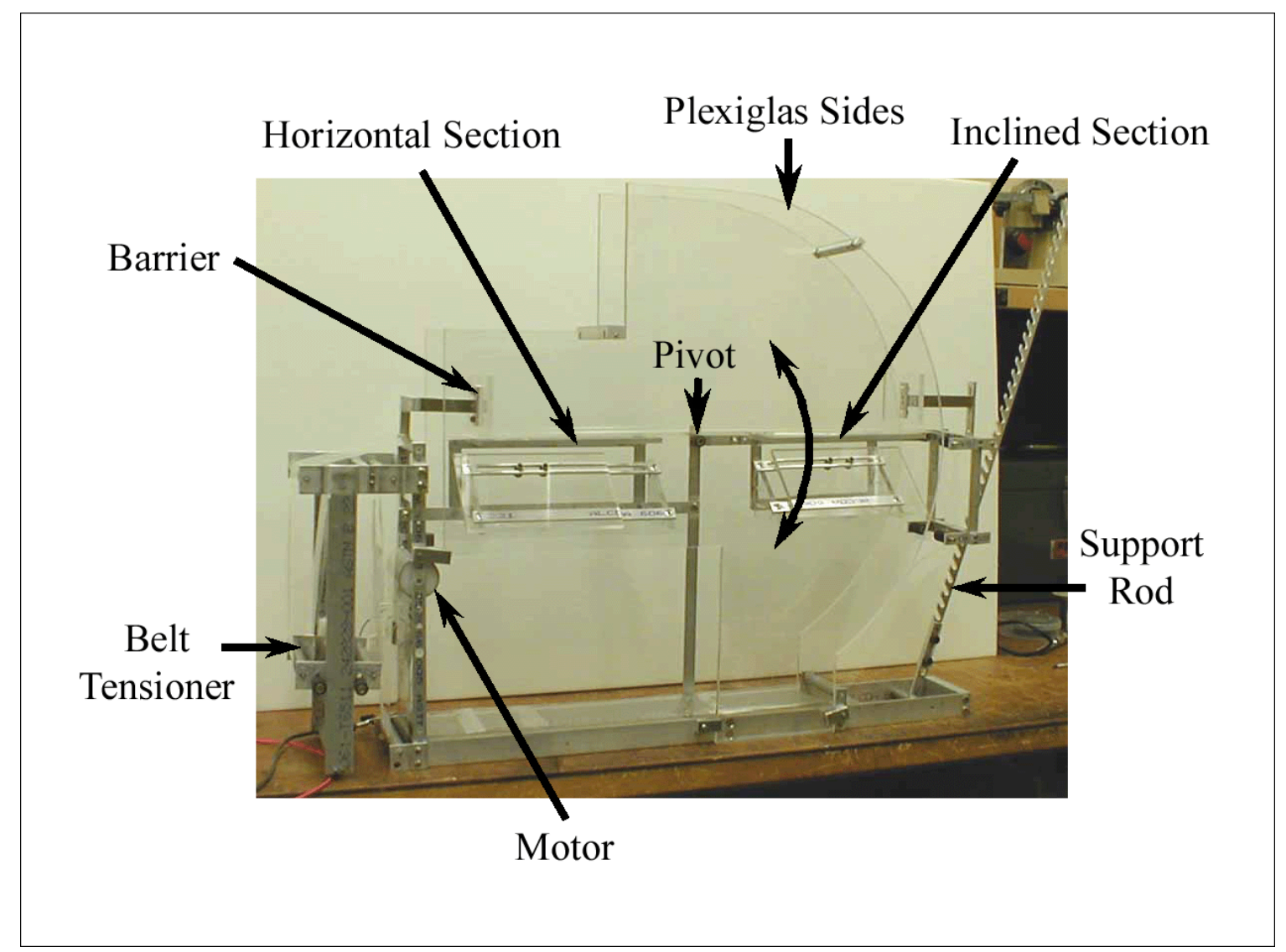

Figure 2-1. The Cockroach Treadmill

The inclined section is supported at one end by a pivot point, which is attached to the frame that supported the horizontal section. The pivot axis is horizontal. At the other end, the inclined section is supported by a support rod. The support rod has several notches in which the inclined section could rest. By changing which notch is supporting the inclined section, changes to the inclination of that section can be made quickly.

A single belt runs through both the horizontal and inclined sections. Therefore, a cockroach moving between the horizontal and inclined sections does not experience a change in surfaces. Miniature rollers guide the belt through the transition area, and ensure that the belt moves smoothly.

During changes to the inclined section, the belt length changes considerably. Moving the inclined sections between its two extreme positions requires the belt to change length 
by about $90 \mathrm{~cm}$. It is for this reason that a belt tensioner is necessary. The entire treadmill has to generate as little friction as possible, so that the motor can move the belt smoothly and at a constant speed. There were, therefore, several difficult design goals that had to be achieved with the belt tensioner.

Both the horizontal section and the inclined sections of the treadmill have mirror holders under them. Mirrors mounted at these locations allow a high-speed video camera to have a simultaneous view of the side and bottom of a cockroach on the treadmill. Consideration to the placement of supports was given during the design of this treadmill, so that supports did not obstruct the view of high-speed video cameras.

A cockroach on the treadmill is unable to escape regardless of the orientation of the inclined section, due to Plexiglas on the sides of the treadmill, and Plexiglas barriers to the front and rear.

Almost all of the components in the treadmill were machined out of aluminum and Plexiglas, which reduced cost and weight.

A DC hobby motor provides power to the belt in the treadmill, and a variable voltage power supply allows belt speed changes to be made.

Ideally, when a cockroach is placed in a treadmill test, the treadmill speed would be adjusted to keep the animal in the same location while the high-speed video cameras record the action. Unfortunately, the cockroaches walked at an erratic speed in the transition area, making it impossible to keep the animal in the same place. The belt was therefore kept stationary during the actual tests. The camera view was focused on the transition area, and the cockroach was filmed running through the frame. 
Circumesophageal Connective Lesioned (CoCL) animals are created by a surgical lesion that disconnects part of the brain from the rest of the nervous system. CoCL animals walk in a continuous tripod gait and walk fine on level surfaces. They even continue to walk when they have run up against a wall. It has been suggested that this surgical lesion shows that the brain centers that promote walking are in the subesophageal ganglion, whereas centers that inhibit walking are in the supraesophageal ganglion [37].

Tests were performed with both intact animals and CoCL animals on the treadmill by researchers in the Insect Movement Behavior Lab. The intact animals were able to maintain their posture, and as a result, were more successful at walking up inclines. The CoCL animals were not able to maintain their posture, which prevented their front tarsal pads from being able to properly engage the substrate on inclines, and had more difficulty walking up inclines [30, 31, 34]. 


\section{Leg Design}

\subsection{Key Features of Cockroach Foot Trajectories}

Cockroach foot trajectories are desirable to imitate because they enable a cockroach to run and surmount small obstacles without active trajectory changes. Each cycle of a leg consists of two phases - the swing phase, and the stance phase. During stance, the foot is on the ground, and the leg is able to help support the weight of the body and propel the body forward. During swing, the foot is off the ground, and the leg is swung forward. In this phase, the leg cannot help to support the weight of the animal and cannot provide propulsive forces. At heel strike, the leg makes the transition from swing to stance, and at toe off, the leg makes the transition back to swing.
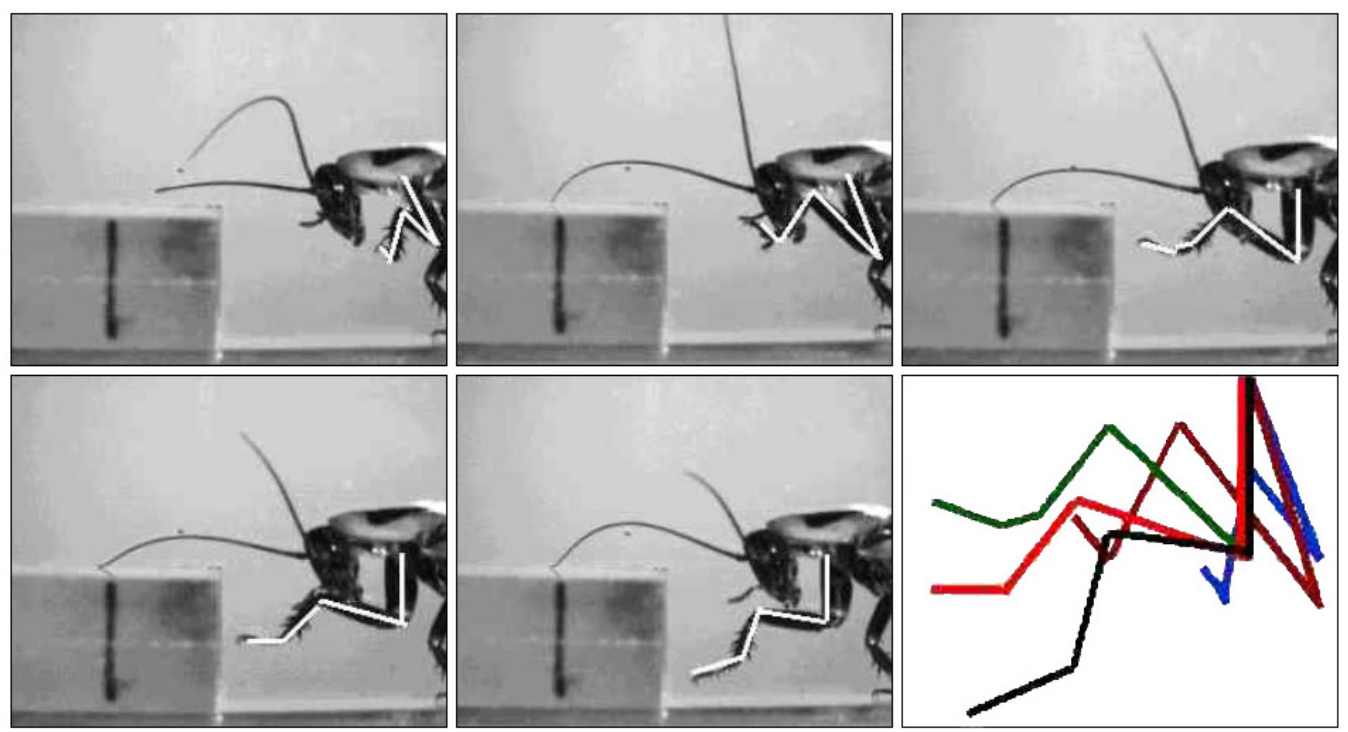

Figure 3-1. Cockroach Front Leg Raised High During Swing (Courtesy of Ritzmann Lab)

Figure 3-1 shows frames from high-speed video from a Blaberus discoidalis cockroach during normal walking. Cockroaches normally walk by lifting their front legs high during swing, which allows them to run over obstacles $60 \%$ of their height without actively changing foot trajectories [44]. While the cockroach would not have been able 
to climb over the $11 \mathrm{~mm}$ obstacle shown in the figure without actively changing its gait, it would have been able to climb a 5.5mm obstacle without doing so [44].

Typically during walking, front legs are used to decelerate the body in the horizontal direction, while middle legs are used to both accelerate and decelerate, and rear legs are used for forward acceleration [12].

The ground reaction forces on the legs toward the end of stance cause a torque to be created about the animal's center of mass. This torque causes the animal's head to be pitched downward. The decelerating forces created by the front and middle legs are used to reverse the downward pitching motion of the head [43].

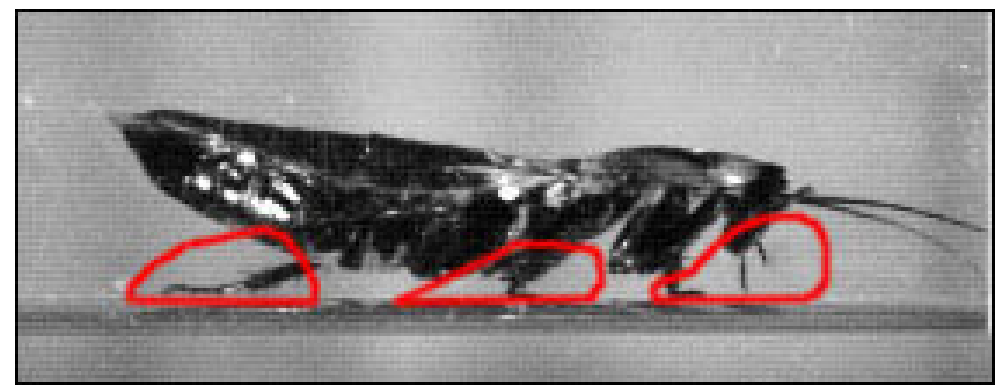

Figure 3-2. Cockroach Footpaths During Walking[7]

A Blaberus discoidalis cockroach is shown walking on a treadmill in Figure 3-2 with foot trajectories overlaid. The stride length of each of the legs is approximately $25 \%$ of the length of the cockroach.

The cockroach normally walks in an alternating tripod gait, in which the front and rear leg on one side moves in phase with the middle leg on the other side of the animal [47]. The remaining three legs form the second tripod. This gait provides a stable base of support for the animal, and is a desirable behavior for the robot. 


\subsection{Previous Approaches in Robotics}

Abstracted cockroach locomotion principles have been implemented in biologically abstracted mechanisms before, such as in RHex [38] and the Whegs ${ }^{\mathrm{TM}}$ series of robots [3].

The Whegs ${ }^{\mathrm{TM}}$ series of robots seek to combine the advantages of wheels and legs. Wheels have allowed vehicles to move very quickly (see Figure 3-3), which is obviously a desirable characteristic in a robot. Wheels also allow a vehicle to be centrally powered, meaning that the vehicle needs only to have a single drive motor to power multiple wheels. As previously mentioned, using a single drive motor allows for a higher powerto-weight ratio, which is also desirable in a robot. Unfortunately, wheels are very poor for traversing terrain that has obstacles or is discontinuous, making them unsuitable for use on a robot that is expected to operate on that kind of terrain.

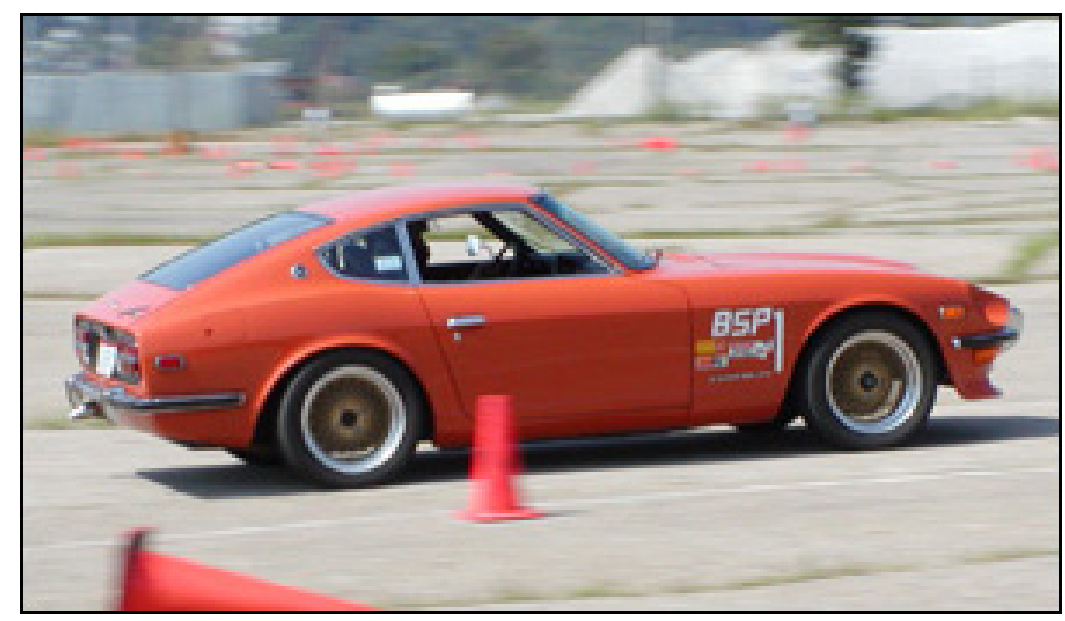

Figure 3-3. A Wheeled Vehicle Moving at a High Rate of Speed

Legs have given animals and robots far better obstacle climbing ability than wheels of the same size. Furthermore, legs give both animals and robots the ability to walk across discontinuous surfaces. For this reason, there are a multitude of legged robots under development at various robotics labs across the world. 
Unfortunately, the use of legs generally requires many more actuators and a much more complex control scheme than wheels. The increased complexity in actuators and control systems allow legged animals and robots to have legs that are specialized for different tasks. For instance, front legs can be optimized for reaching or other dexterous behaviors that are not required of the middle and rear legs. Legs can also perform complex movements that wheels can't, such as searching for a foothold. In addition, legs can switch between performing different tasks, whereas wheels are fixed in a rotary motion.

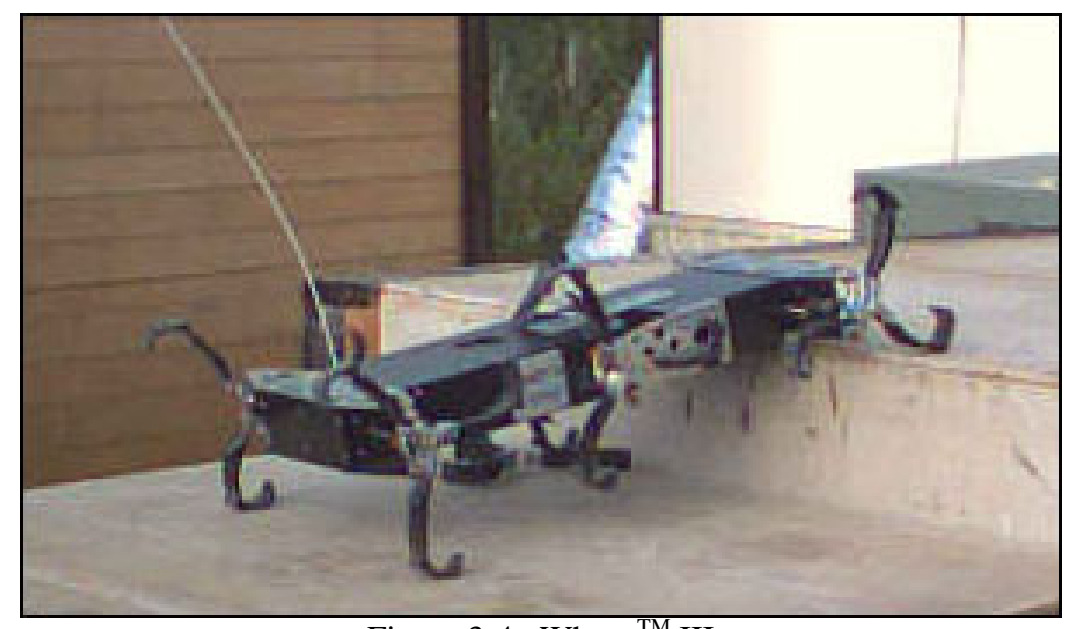

Figure 3-4. Whegs ${ }^{\mathrm{TM}}$ III

Whegs ${ }^{\mathrm{TM}}$ vehicles (see Figure 3-4) are able to combine the advantages of wheels and legs. Instead of using wheels, they use a hub with three spoke-like appendages to make contact with the ground. This gives the vehicle superior obstacle climbing ability over wheels of the same diameter, without the complexity in actuation and control that legs would require. It also allows the vehicle to be centrally powered, allowing it to have a high power-to-weight ratio and to move as quickly as it would be able to with wheels.

The simplicity in actuation and control of Whegs ${ }^{\mathrm{TM}}$ has the same disadvantages as it does with wheels. Complex movements aren't possible with Whegs ${ }^{\mathrm{TM}}$ because the 
spoke-like appendages are fixed in a rotary motion. In addition, it is very difficult to have Whegs ${ }^{\mathrm{TM}}$ specialized for different tasks on the same robot, because they generally have to be of similar diameter.

\subsection{4-Bar Mechanisms}

The ideal legs for a robot would have the advantages of both Whegs ${ }^{\mathrm{TM}}$ and legs. A vehicle that could be centrally powered, while at the same time having legs that can be specialized for different tasks has the potential to be very useful in a number of applications across a wide variety of terrains.

One way that these goals can be made possible is through the use of 4-bar mechanisms. 4-bar mechanisms are linkage mechanisms that allow the transformation between different types of motion. For instance, the linear motion of a piston in an automobile engine can be transformed into the rotary motion of a driveshaft through a 4bar mechanism. These mechanisms have been used in many applications such as automobile windshield wipers and pantographs, the latter of which have been in use for hundreds of years. 4-bar mechanisms were also used as legs on MechaRoach I, and cockroach-like mobility was observed as a result [7].

Like MechaRoach I, MechaRoach II uses 4-bar mechanisms that transform the rotary motion from driveshafts into cockroach-inspired foot trajectories.

There are several different types of 4-bar mechanisms, such as double-rocker, doublecrank, and slider-crank. The legs of MechaRoach II are crank-rocker mechanisms. 4-bar crank-rocker mechanisms consist of a ground link (the chassis of the robot), a crank (driven by the driveshaft), the rocker link, and the coupler (which is analogous to the tibia 
of a leg). These links are labeled on a picture of a front leg in Figure 3-5. During the analysis of these mechanisms, the terms tibia and coupler will be used interchangeably, because they both refer to the same link of the mechanism.

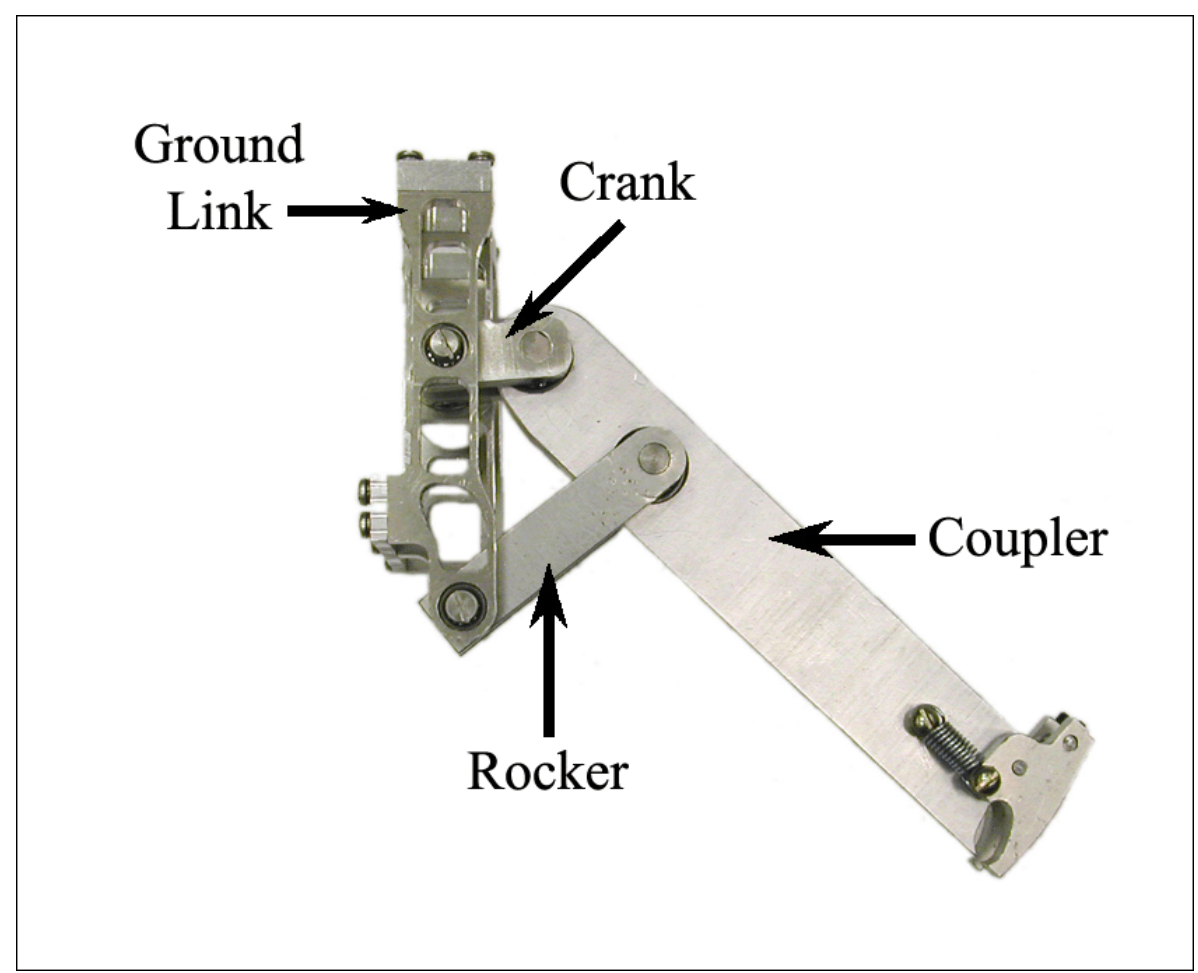

Figure 3-5. A Front 4-Bar Mechanism Leg

4-bar mechanisms can produce three-dimensional movement. However, they are generally designed to be planar because the common techniques used to design them apply only to planar mechanisms. The leg mechanisms on MechaRoach II are planar, and they only attempt to recreate features of cockroach footpaths as viewed in the sagittal plane.

Lateral movement of the cockroach's body during running adds to the animal's stability [19]. However, the robot is not able to compensate for lateral movements of its body with movements of its legs. It is therefore undesirable for feet of the robot to move laterally during stance. When the robot is climbing, if a foot gains a foothold, this foothold may be lost if the foot moves laterally. It is for this reason that the 4-bar leg 
mechanisms are oriented vertically in addition to being planar. This orientation prevents lateral movement during swing and stance. The planes the leg mechanisms move in are parallel to the sagittal plane of the robot.

The usage of 4-bar mechanisms as legs enables a combination of many of the advantages of Whegs ${ }^{\mathrm{TM}}$ and legs. Different legs of the same robot could be specialized for different tasks, just as legs are. The different legs could have cockroach-inspired foot trajectories in the sagittal plane, which would be impossible with Whegs ${ }^{\mathrm{TM}}$. The mechanisms allow the robot to be centrally powered, which can allow a vehicle to move very quickly and have a high power-to-weight ratio. Finally, the leg mechanisms allow other mechanisms (such as claws, suction cups, magnets, or other end-effectors) to be mounted to the end of them, which would be difficult with Whegs ${ }^{\mathrm{TM}}$.

It would have been possible to use the leg mechanisms from MechaRoach I on MechaRoach II. Unfortunately, the mechanisms used on MechaRoach I have poor mechanical advantage characteristics. In addition, the vertical displacement of the chassis during walking is too large to be suitable for a climbing robot. Therefore, new leg mechanisms were required to be designed.

\subsection{Mechanism Design Goals}

In order for a 4-bar mechanism to properly function as a leg, it must simultaneously balance several conflicting design goals. Many tradeoffs had to be made during the design process to ensure that as many of these goals could be satisfied as possible.

Mechanical advantage is a ratio of the output force to the input force. During swing, the mechanical advantage could be much lower than during stance because the leg should 
be unloaded in that phase. The mechanical advantage of the mechanism during stance must be large enough so that during climbing, the robot has enough torque to propel the robot forward. Redesigning the leg could be the answer to an insufficient mechanical advantage. Alternatively, a larger gear reduction could be placed between the drive motor and the legs. Unfortunately, a larger gear reduction would reduce the overall speed of the robot. The usage of a more powerful motor could also be used to solve an insufficient mechanical advantage problem. However, during most of the design phase, the drive motor was already on hand. The legs were designed with that motor in mind, because the motor was easily the single most expensive component in the robot. Therefore, when leg mechanisms were shown to have a mechanical advantage that was too low, the legs were redesigned.

Cockroach-like foot trajectories must be created when the mechanism is viewed in the sagittal plane. The resulting footpath must exhibit the desired features learned from cockroach research, such as the previously described high reaching of the front legs during swing. Ideally, the cockroach's stride length should also be duplicated proportionately.

During stance, the horizontal foot velocity should be as constant as possible for the purposes of leg coordination. At any given time, many legs are on the ground at the same time, and they all have to work together to propel the robot forward. The horizontal foot velocity during stance should be similar for all of the legs on the same robot for the same reason.

Also during stance, the vertical foot displacement should be minimized. Any vertical foot displacement during stance will result in vertical movement of the chassis. 
Movement of the chassis away from the substrate needs to be minimized on a climbing robot, to minimize the required attachment forces.

Each of the legs must reach the same distance below the body during stance. If one leg does not reach as low during stance as the other two legs in its respective tripod, the load will not be divided appropriately among those legs. The shorter leg will be supporting less of the load, while the other legs will be supporting more.

During swing, the foot must remain above any level that it would be in during stance, in order to ensure ground clearance while the foot is swung forward. Otherwise, the foot will make contact with the ground during swing and push the robot in the wrong direction. The ground clearance during swing should be high enough to give the robot some obstacle climbing ability.

The 4-bar mechanism must mount to the chassis at two locations. One location is where the driveshaft exits the chassis and powers the crank of the mechanism. The other location is where the mechanism's rocker connects to the chassis through a passive pin joint. These mounting locations must connect to the chassis at locations that are possible to construct. An example of an unacceptable mounting location would be one that is below the ground.

The crank and the rocker of the mechanism cannot overlap at any point in the leg's trajectory when viewed in the sagittal plane. Because of the way that the legs must be constructed to support the large expected loads, the components of the crank and rocker links must be located in the same plane. Therefore, any overlap between those two links will cause a collision. 
The mechanism must trace one complete stance-swing cycle for one revolution of the crank. With the crank rotating at a constant speed, the mechanism should spend the majority of its time in stance, and a minimal amount of time in swing. This will ensure overlap between the two tripods during an alternating tripod gait.

The intent of the design was to construct a claw at the endpoint of each leg. This would allow the robot to climb mesh or coarse substrates. The mechanism design is required to allow these claws to be easily engaged and disengaged. Extreme changes in tibia orientation would prevent the claw from being engaged and disengaged easily. Extreme changes in tibia orientation will also force the claw to rotate by large amounts, which will prevent the claw from maintaining attachment to any foothold it has found.

Finally, the leg mechanisms must keep the chassis of the robot close to the substrate. Keeping the body closer minimizes the forces necessary to keep the robot attached to a vertical surface. It is desirable and may be even necessary to keep the chassis close to the substrate due to the gripping limitations of the robot.

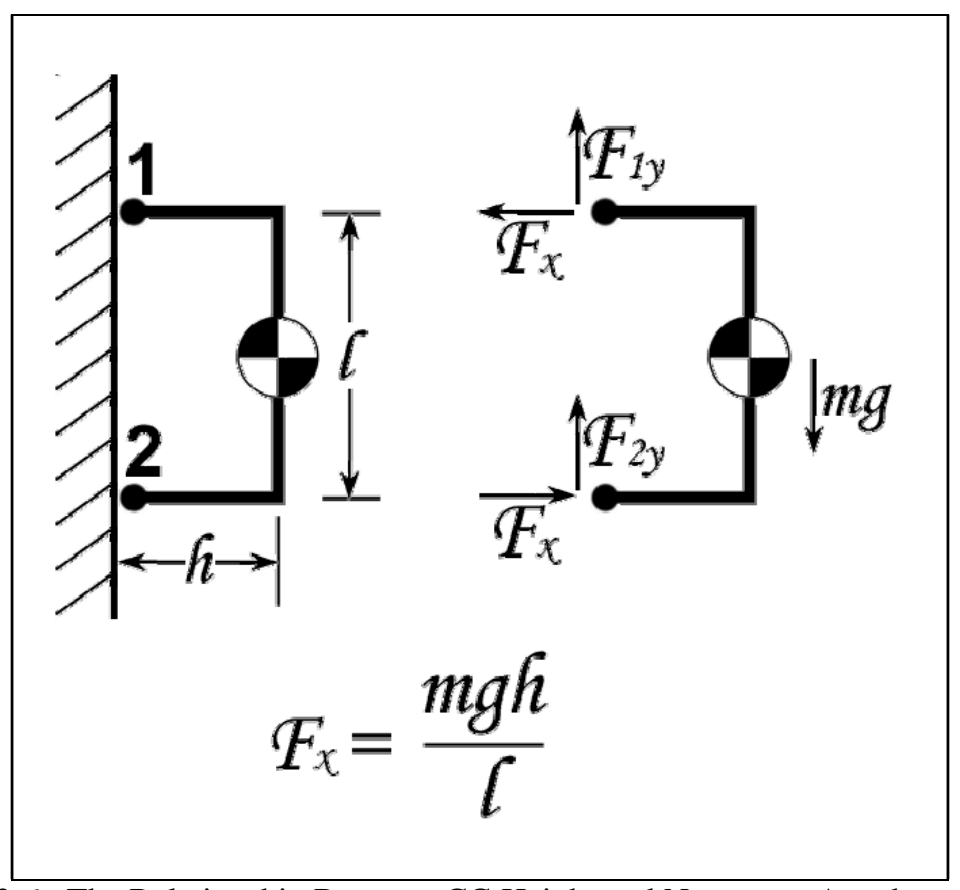

Figure 3-6. The Relationship Between CG Height and Necessary Attachment Force 
Figure 3-6 is a diagram of a hypothetical robot that is attached to a vertical substrate at two locations. The right side of the figure is a free body diagram. The center of gravity of the robot is located distance $h$ from the substrate, and midway between the two attachment points, which are distance $l$ apart. $F_{X}$ is the attachment force required at the upper attachment point, and can be shown to be equal to $\mathrm{mgh} / \mathrm{l}$. This is the force that must be generated by the end effector at the upper attachment point for the robot to remain attached to the substrate. If the robot is unable to generate that force, it will fall. The necessary attachment force is proportional to $h$, which explains why it is necessary to minimize $h$ in order to minimize the necessary attachment force.

\subsection{The Mechanism Design Method}

The design of the leg mechanisms had five distinct phases that were used iteratively. The phases were graphical synthesis, analysis using the Working Model software package, Lego prototyping, analysis using the Mechanism Analyzer program, and evaluation of the finished leg.

\subsubsection{Graphical Synthesis}

There are many graphical and analytical methods for generating 4-bar mechanisms. Some examples are Three-Position Motion Synthesis, Three-Position Path Generation, and Path Generation With Prescribed Timing [39].

Three-Position Graphical Synthesis was chosen because of its ease of use. Several mechanisms could be generated very quickly, meaning that large amounts of time did not 
have to be devoted to this stage of the process. However, it is a pencil and paper method, so patience was required with a ruler and a protractor.

When using this method, there are a number of free choices. Free choices are variables that can be chosen arbitrarily, and which everything else is calculated from. A mechanism that has poor characteristics can be redesigned by changing one or more of the free choices. The free choices include the location of the two pivot points on the tibia (where passive pin joint connections are made with the crank and rocker) and the output locations or orientations of the coupler. When designing the middle leg, two of the output positions and orientations might be the position and decelerating orientation of the tibia at heel strike, and the position and accelerating orientation at toe off (see Figure 37). The lengths of the various links and position of joints have various effects on the output of the mechanism. Most of these effects are not intuitive.

Because this is a pencil and paper method, visualizing how the mechanism will move is very difficult. Many mechanisms that appeared on paper to have desirable characteristics turned out to be obviously poor leg mechanisms when modeled in later stages of the design process. 


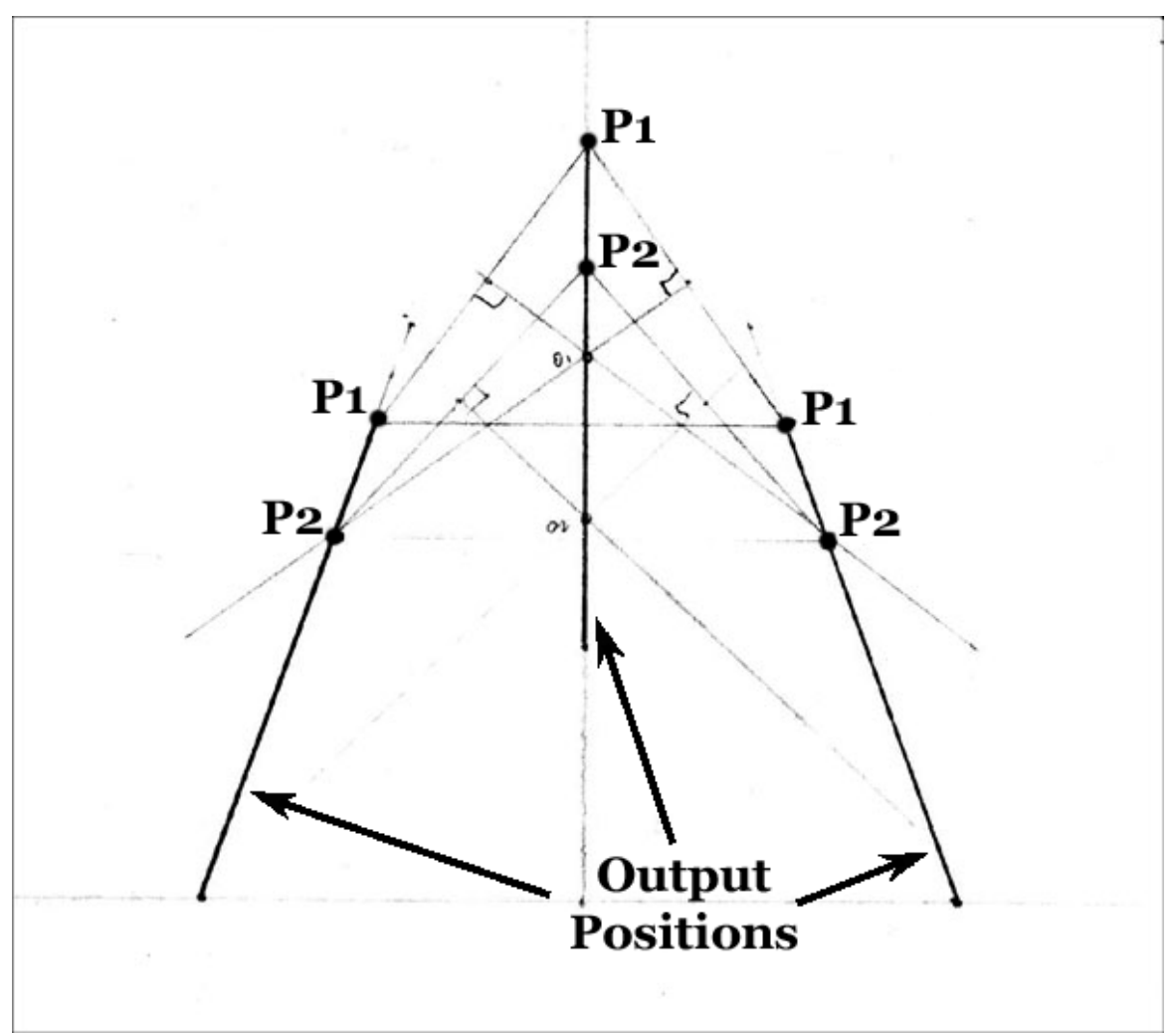

Figure 3-7. Three-Position Graphical Synthesis

First, the three output positions and orientations for the tibia were chosen. Next, the two pivot locations $\left(\mathrm{P}_{1}\right.$ and $\left.\mathrm{P}_{2}\right)$ on the tibia were chosen. Lines were drawn between the various $\mathrm{P}_{1} \mathrm{~s}$ on the three output positions. Then lines were drawn between the various $\mathrm{P}_{2} \mathrm{~S}$ on the output positions.

Next, perpendicular bisectors were drawn through the lines drawn in the previous step. The intersection of the perpendicular bisectors defined the ground pivot locations. The ground pivots are the locations where the crank and the rocker make their connections to the chassis of the robot.

Finally, the exact positions of these various points were measured so they could be used in subsequent phases of the design process. 
Designing a candidate mechanism using this method only takes a few minutes, and was used to generate mechanisms close to what could be used on the robot. These mechanisms were then adjusted in the other design phases.

\subsubsection{Working Model 2D}

The Working Model 2D software package was produced by Knowledge Revolution. It allows mechanisms to be very quickly modeled. It has a drag-and-drop interface that allows the user to model solid objects, add joints (revolute, prismatic, etc), add motors, and view the mechanism in motion. Figure 3-21 is a screenshot from this software package.

The software was chosen for use in the mechanism design process because of availability, and because its capabilities and limitations were already known. One limitation is that the software can only model objects in two dimensions. However, since the leg mechanisms were planar, this limitation did not prevent the software from being useful.

As stated previously, all of the 4-bar mechanisms used for the legs were to function in planes parallel to the sagittal plane of the robot. All of the work performed with this software was constrained to those planes.

Once a candidate mechanism had been created using graphical synthesis, the mechanism could be very quickly duplicated in Working Model. The mechanism could then be viewed in motion, which the paper-and-pencil graphical synthesis method obviously can’t provide. 
Once viewed in motion, most candidate mechanisms could easily be eliminated. Some mechanisms generated extreme changes in orientation of the tibia, or had foot velocity characteristics so undesirable that they could be identified without numerical analysis. Many mechanisms physically could not operate with a continuously rotating crank (in the colloquial, they would "jam"). Some of the mechanisms could immediately be eliminated due to overlap between the crank and rocker links - As previously explained, overlap between those two links will cause mechanical collisions, and is therefore completely unacceptable under any condition.

At this stage, many candidate mechanisms looked as though they had the desired characteristics and could actually be used on the robot. In later stages, most of those mechanisms would be found to be poor leg mechanisms. When a mechanism with favorable characteristics was found, the software could be used to make small changes to the geometry of the mechanism. The resulting motion could then immediately be viewed. Being able to quickly make changes and immediately view the resulting footpath was an enormous advantage over graphical synthesis. Changes often only took seconds to make, so several changes could be made, and the results could be compared.

Unfortunately, numerical analysis of the mechanisms wasn't possible or was very difficult with Working Model. It was therefore just used to visualize the motion of the various candidate mechanisms to eliminate ones that were obviously undesirable, and to adjust ones that looked close to being usable. However, it was insufficient for full analysis of any of the mechanisms. 


\subsubsection{Lego Prototyping}

After the initial mechanisms for the legs of the robots were designed using graphical synthesis and Working Model, they were constructed from Legos borrowed from the Autonomous Robotics Class in the Electrical Engineering and Computer Science department at Case Western Reserve University.

Physical models provide an intuitive understanding of the properties of physical objects that software cannot provide. For instance, a hand crank was used to actuate the Lego model of each leg, which made obvious how much physical effort was required to power the leg. Required effort is just one example of a characteristic that was not possible to learn from a software model, even if required torque numbers had been available. 


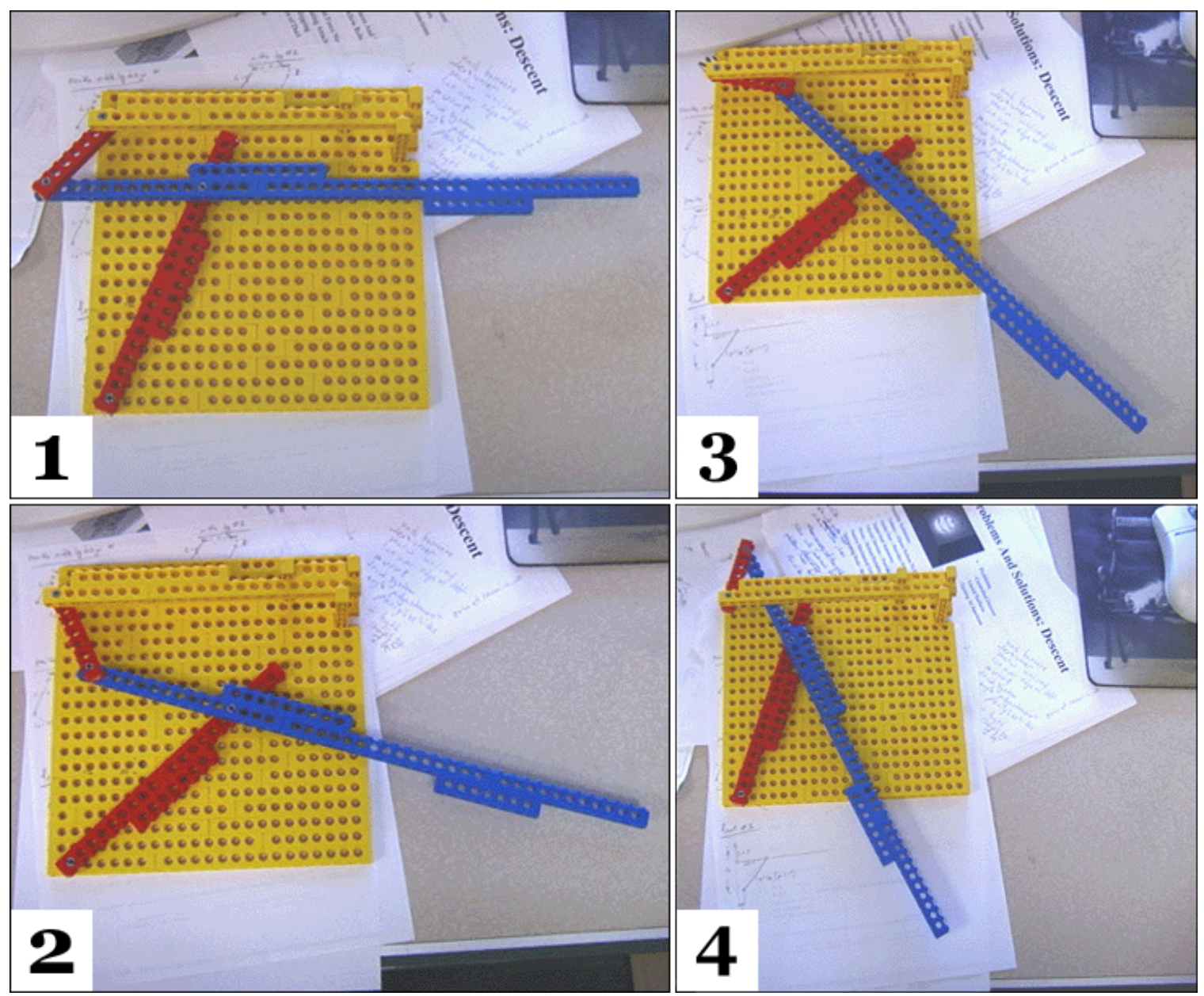

Figure 3-8. Lego Prototype of Front Leg

Figure 3-8 shows a Lego prototype of a front leg candidate mechanism in four different positions, with the front of the robot being toward the right. The crank (the shorter, red link) is rotating counter-clockwise. The blue link is the tibia. The longer, red link is the rocker. All of the joints are pin joints.

In position 1, the front leg is raised high in swing. Again, raising high during swing allows the leg to reach over obstacles without active trajectory changes. In position 2, the foot is being swept downward. In position 3, the tibia has finished sweeping downward, and the leg begins to draw it straight backward. In position 4, the leg continues to draw the foot backward, and prepares to transition back into swing. 
In addition to allowing for an intuitive feel of the mechanisms, the usage of Legos had many other advantages. Legos allowed prototypes to be built in minutes, without consuming any material. Creating prototypes by machining material is more timeconsuming. The prototype legs could have been constructed with aluminum or plastic, but large amounts of material would have been consumed in the process had large numbers of prototypes been built.

Once several legs had already been constructed for evaluation on the actual robot, prototyping with Legos was stopped. Many of the leg redesigns were very similar to legs that had already been built, and therefore Lego models of them were not necessary.

\subsubsection{The Mechanism Analyzer Program}

Although the techniques used in the previous phases of the design process were informative and invaluable, none of them provided any numerical analysis. For instance, the mechanical advantage of each of the legs in any position could not easily be determined by any of the processes described in the previous sections. After the first legs were constructed for evaluation on the actual robot, it became obvious that numerical analysis would be helpful.

The program was written in $\mathrm{C}$ and compiled in Metrowerks Codewarrior version 9. It gradually developed throughout the design process, and the final version of the code can be found in Appendix A.

The inputs to the program were the geometry of the linkage. The geometry included the locations of the ground pivots and the lengths of all of the links in the mechanism. The program simulated the crank being driven at a constant $1 \mathrm{rad} / \mathrm{sec}$, and outputted data 
points every time the crank rotated 1 degree (360 total). The leg mechanisms complete one swing-stance cycle for every 360-degree rotation of the crank. Instantaneous outputs included the position of all of the joints, the position of the foot, the horizontal and vertical velocity of the foot, and the mechanical advantage of the mechanism.

Each mechanism functioned in a vertical plane, parallel to the sagittal plane of the robot. All of the analysis done by the program was with respect to that plane, and the coordinate system was two-dimensional.

The reference frame was attached to the chassis of the robot, so for the purposes of this analysis, the chassis was fixed and motionless. Therefore, the locations of the linkage ground pivots were also fixed. The crank was connected to the chassis through the driveshaft at one of the ground pivots called $\mathrm{O}_{1}$ (see Figure 3-9), which was also the origin of the coordinate frame. The other end of the crank connected to the tibia at a passive pin joint called $\mathrm{P}_{1}$. The crank was being driven, so $\mathrm{P}_{1}$ 's instantaneous position and velocity were known. $\mathrm{P}_{2}$ was the location of the joint where the tibia and rocker were connected. The other end of the rocker was connected to the chassis through a passive pin joint at the other ground pivot, called $\mathrm{O}_{2}$. 

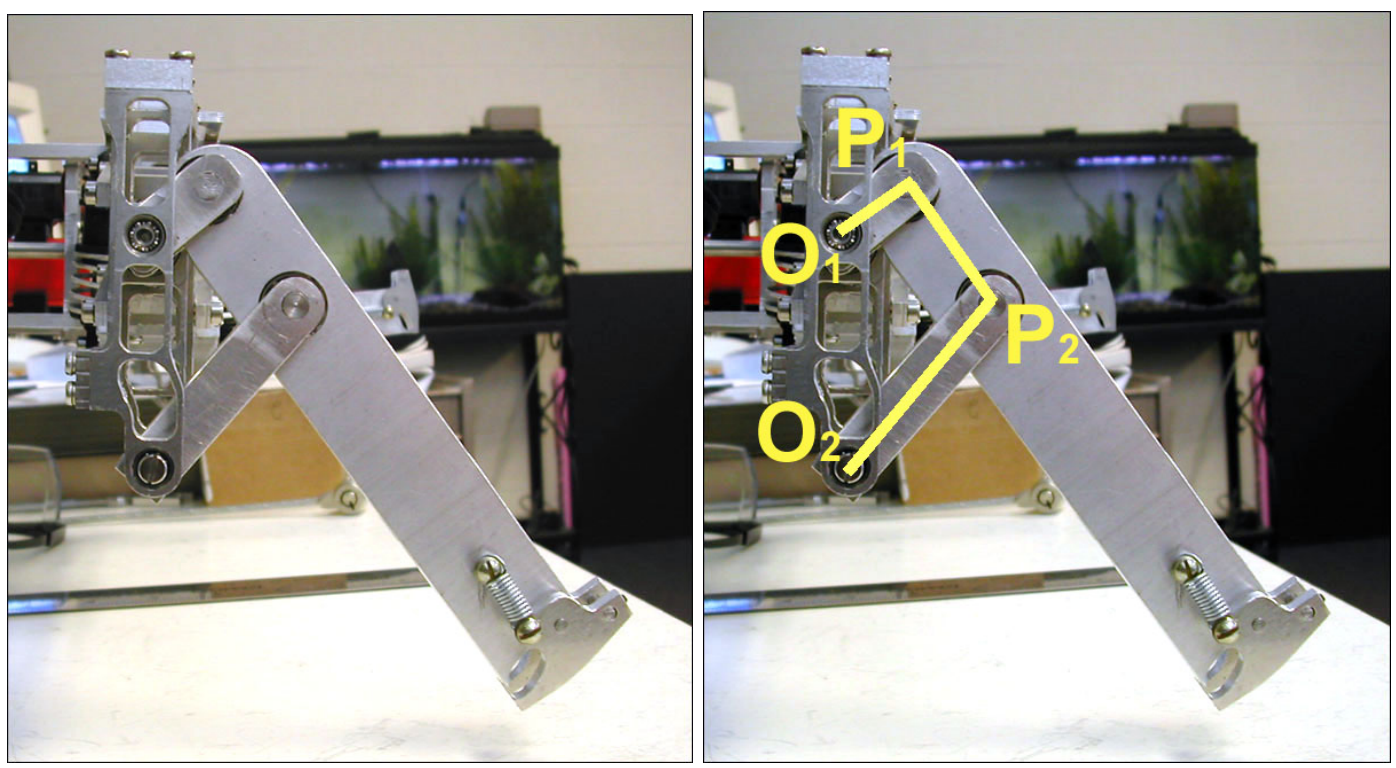

Figure 3-9. Diagramed Front Leg Mechanism

Once the crank's position was specified, the only unknown joint position was that of $\mathrm{P}_{2}$. $\mathrm{P}_{1}$ and $\mathrm{P}_{2}$ were on the same rigid body (the tibia), and therefore at a fixed distance away from each other. Since the instantaneous location of $\mathrm{P}_{1}$ was known, the set of all possible locations where $\mathrm{P}_{2}$ could be defined a circle with the center located at $\mathrm{P}_{1}$. Similarly, $\mathrm{P}_{2}$ and $\mathrm{O}_{2}$ were located on the same rigid body (the rocker) so they must be located at a fixed distance away from each other. Since the position of $\mathrm{O}_{2}$ was fixed, the set of all possible locations for $\mathrm{P}_{2}$ defined a circle with the center located at $\mathrm{O}_{2}$. The instantaneous position of $\mathrm{P}_{2}$ could be found at the intersection of those two circles (see Figure 3-10).

The intersection of two circles generally occurs at two different points, so the solution had to be assumed. The correct solution could be assumed to either be the intersection closest to the front of the robot (as in the case of Figure 3-10), or the one closest to the top of the robot. If the wrong solution was chosen, the foot velocity direction and magnitude would be completely different than expected, making it easy to determine whether the assumption was correct or not. 


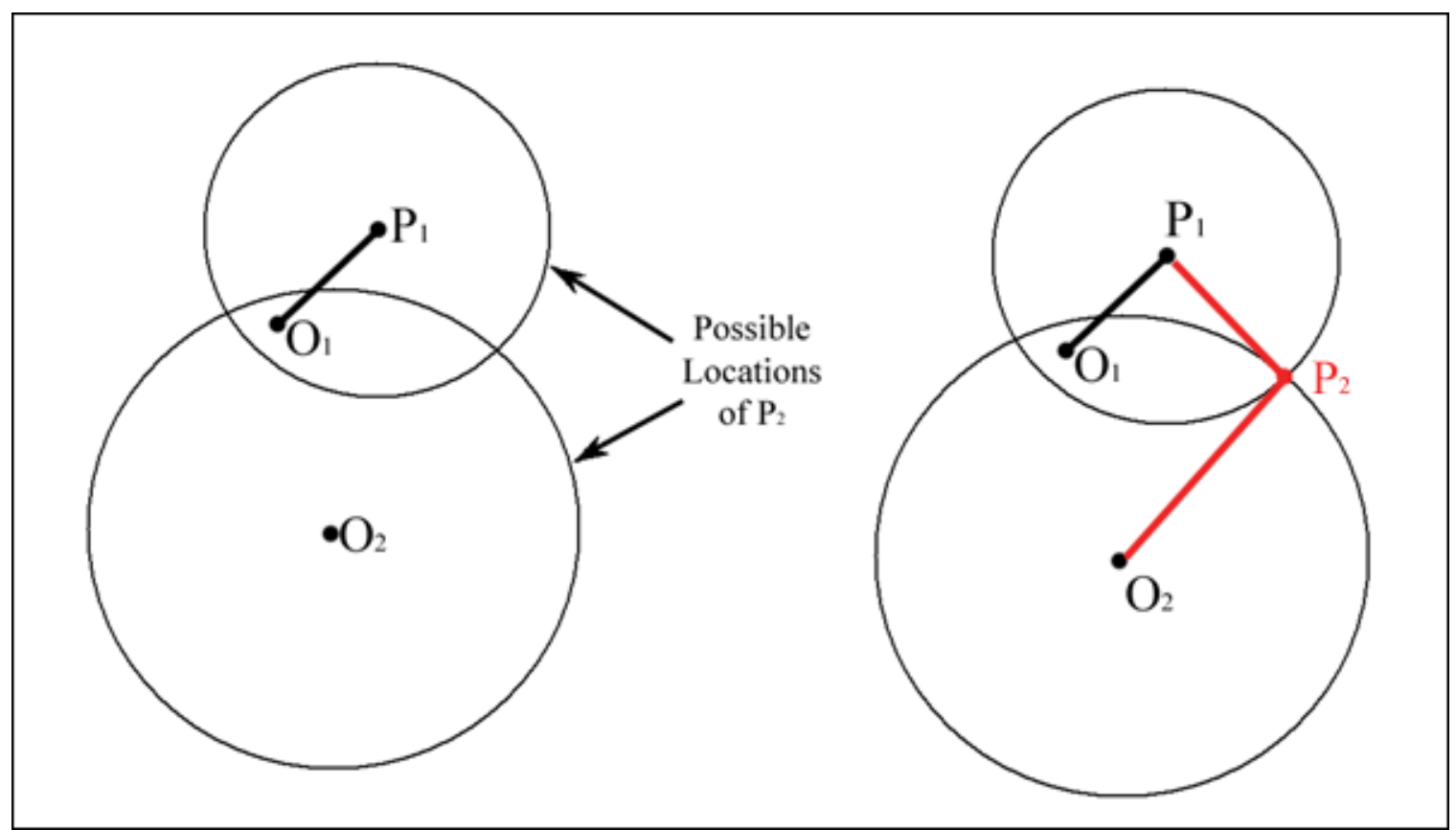

Figure 3-10. Finding the location of $\mathrm{P}_{2}$

Once the locations of all of the joints were found, vectors defining the crank ( $\left.\mathrm{r}_{1}\right)$, the coupler $\left(\mathrm{r}_{2}\right)$ and the rocker $\left(\mathrm{r}_{3}\right)$ could be created.

Next, the instant center of the tibia with respect to the ground link (the chassis of the robot) was located. An instant center is the location where all of the points on a rigid body could be considered to be rotating around at a particular instant in time. A rigid body in pure translation would have an instant center located at an infinite radius away.

For instance, consider the simplest case, which is described in Figure 3-11. A disk is rotating about an axle through its center with velocity $\omega$. Any two arbitrary points A and $\mathrm{B}$ on the rigid body must be rotating about the center, and therefore the center is its instant center (IC). 


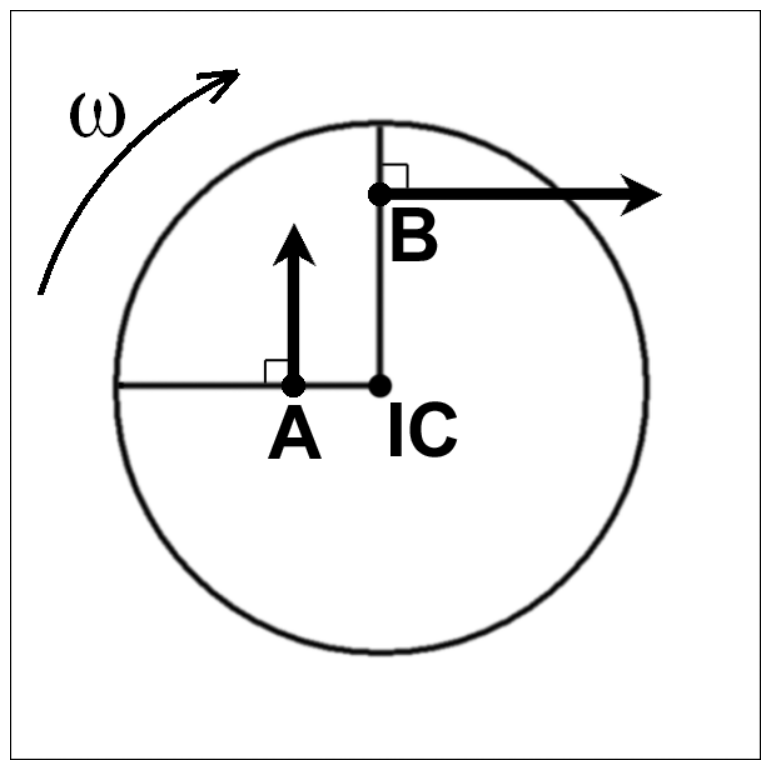

Figure 3-11. Finding Instant Centers

Now consider the case in which it is unknown about which point the rigid body is rotating. If the velocity of points A and B in Figure 3-11 are known (represented by arrows), it is very simple to determine the body's instant center. Perpendicular lines to the velocity vectors could be drawn through points $\mathrm{A}$ and $\mathrm{B}$, and the intersection of these perpendicular lines defines the location of the instant center.

This simple method for locating the instant center of a rigid body can be used for the tibia as well. The directions of the velocity vectors for two points on the tibia are known. Consider the front leg mechanism in Figure 3-9 again. Points $\mathrm{P}_{1}$ and $\mathrm{P}_{2}$ are located at fixed points on the tibia. $\mathrm{P}_{1}$ is also a fixed point on the crank, so it must be rotating about $\mathrm{O}_{1}$. Therefore, the velocity vector has to be perpendicular to the crank, and a line perpendicular to the velocity vector would lie along the crank.

Similarly, $\mathrm{P}_{2}$ is a fixed point on the rocker, and it must be rotating about $\mathrm{O}_{2}$. A line perpendicular to its velocity vector lies along the rocker. Therefore, the intersection of the lines that lie along the crank and the rocker must be the instant center of the tibia (see Figure 3-12). 


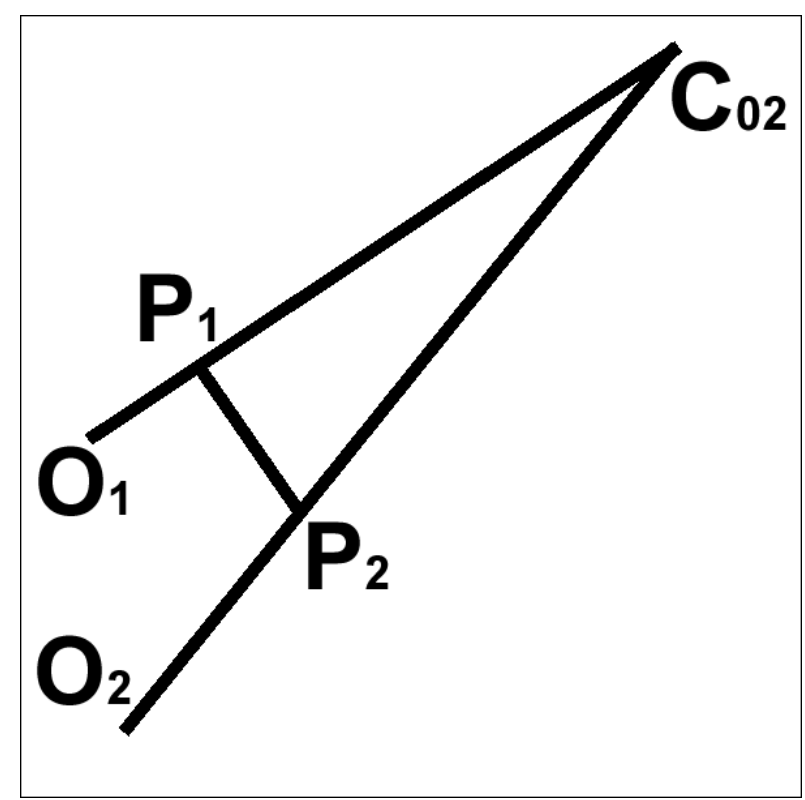

Figure 3-12. Finding the Tibia’s Instant Center

Locating the instant center for each position of the crank is relatively easy to calculate, and the program is able to export the instant center location vs. crank position.

It was initially thought that the location of the tibia's instant center $\left(\mathrm{C}_{02}\right)$ would give an indication of the mechanical advantage of the mechanism. To explain, the mechanism in Figure 3-9 was recreated as a line drawing in Figure 3-13. All of the points on the crank were rotating about $\mathrm{O}_{1}$. And at that instant, all of the points on the coupler were rotating about its instant center - so at that particular instant, it could be imagined that the crank and the coupler were like two gears in mesh at point $\mathrm{P}_{1}$. 


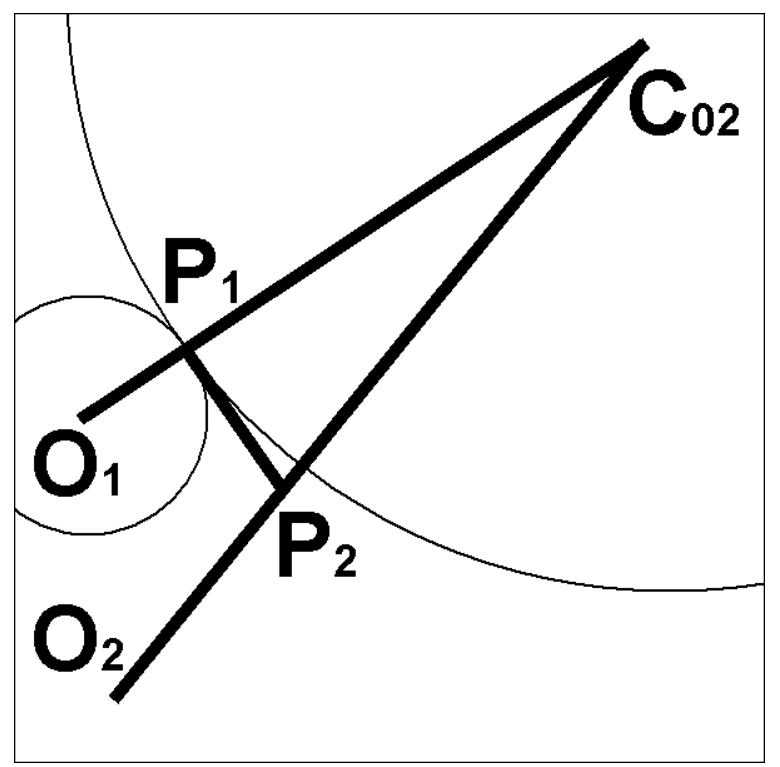

Figure 3-13. The Crank and Coupler are like Two Gears in Mesh

The mechanical advantage of two gears in mesh is very easy to determine, because it is simply a ratio between the radii of the two gears.

The distance between $\mathrm{P}_{1}$ and the instant center reached infinity at two locations during each cycle of the leg. These two locations corresponded to points at which the crank and rocker are parallel, and the tibia is purely translating.
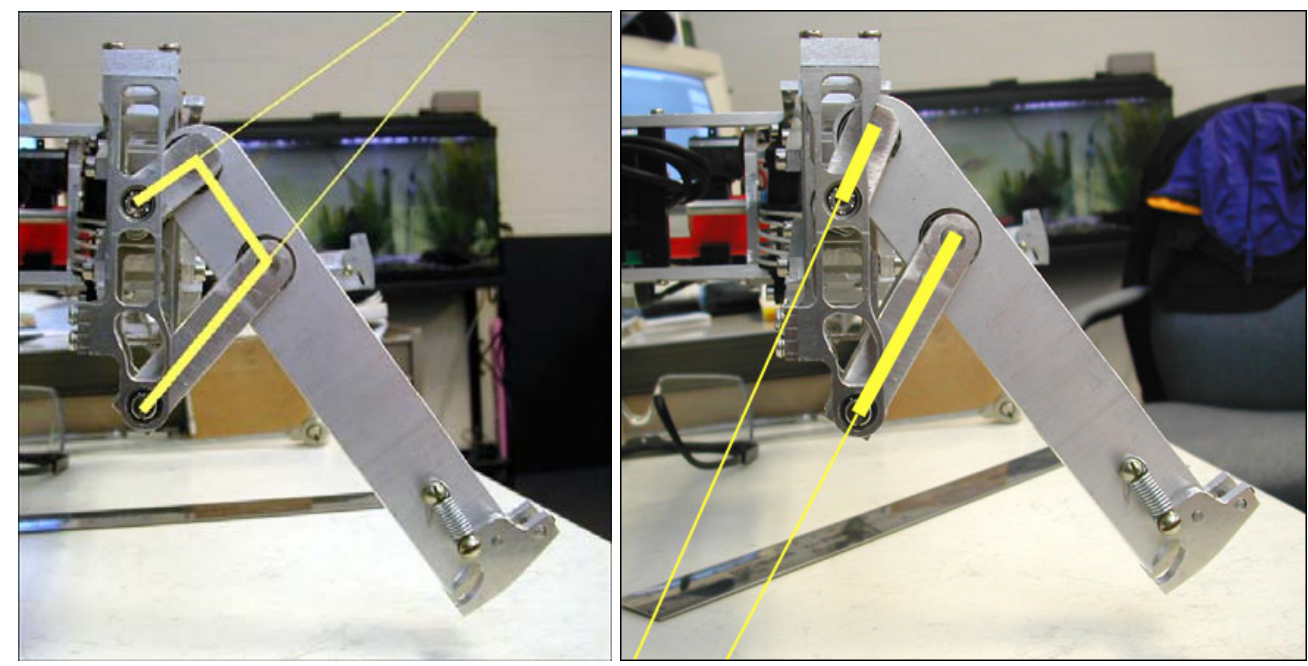

Figure 3-14. Instant Center Locations Move Discontinously

Figure 3-14 shows the mechanism immediately before and immediately after the instant center went to an infinite distance away. The crank was rotating counter- 
clockwise in the pictures. As the crank rotated, the instant center went to infinity out of the frame on the upper right and then it reappeared out of the frame at the lower left.

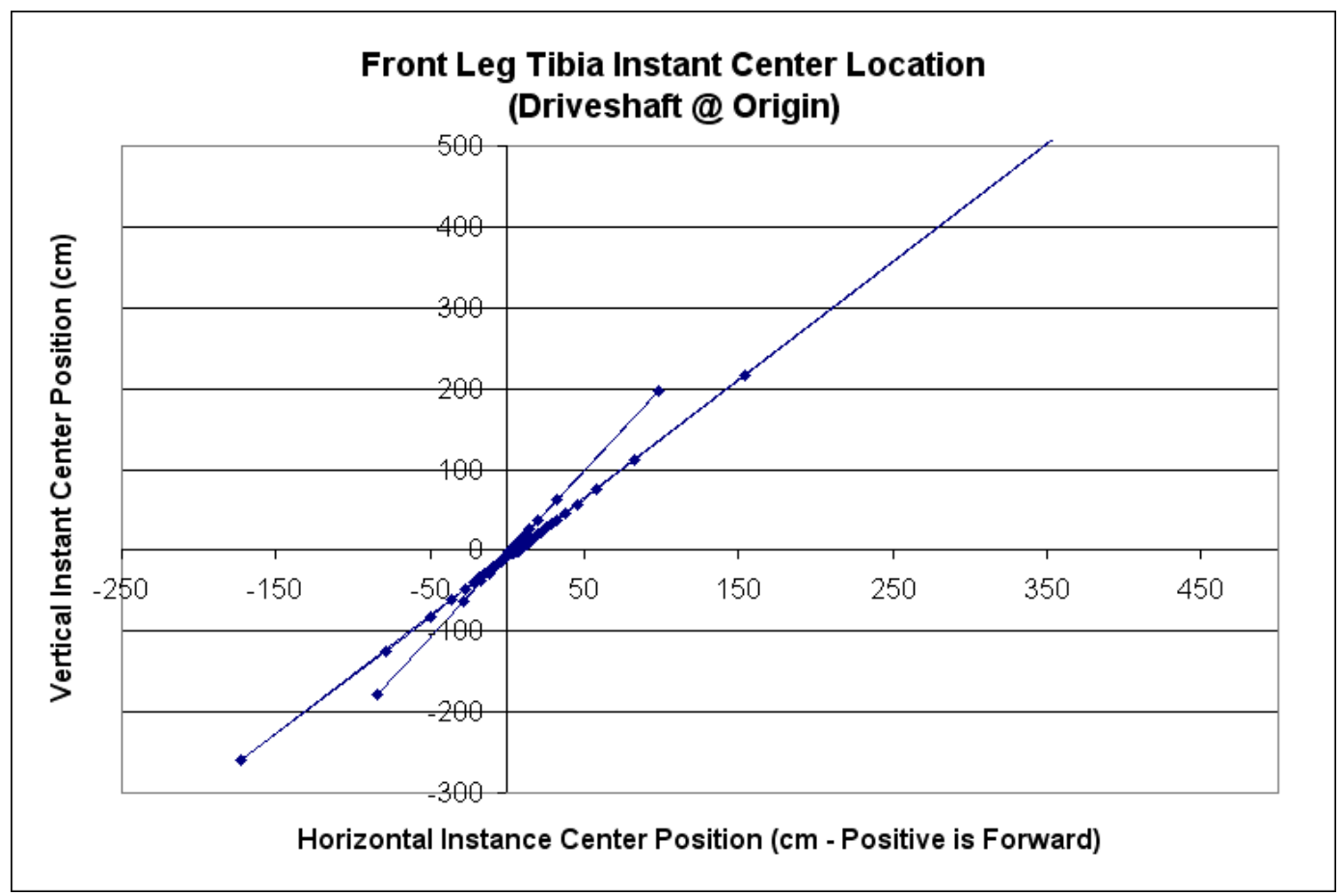

Figure 3-15. Front Leg Instant Center Positions

Figure 3-15 shows a plot of the position of the instant center at various points through a cycle of the mechanism. The origin of the coordinate frame was located at the driveshaft that is driving the mechanism, and the coordinate frame was located in a plane parallel to the sagittal plane of the robot. The program was carefully written in order to avoid doing calculations of the instant center's position exactly at the point where the tibia was purely translating. It is for this reason that the instant center moved very far from the origin, but never actually was plotted at an infinite distance away. 


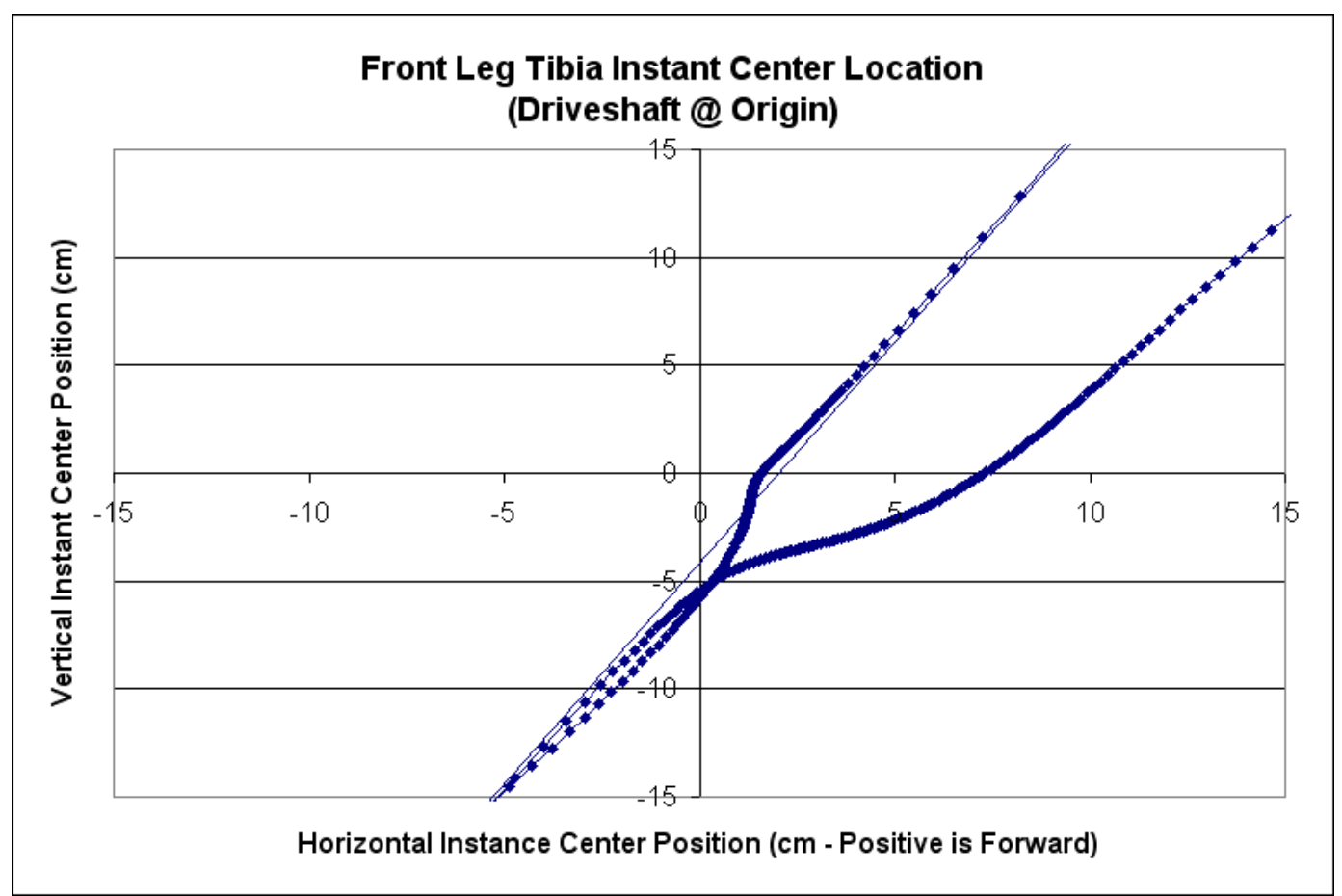

Figure 3-16. Zoomed View of Front Leg Instant Center Positions

Due to the large magnitudes in the distance of the instant center from the origin, it is difficult to see how the instant center moved when it was near the origin. Figure 3-16 shows a more focused view of the previous figure.

The instant centers primarily lay along a corridor that ran through the lower left quadrant and upper right quadrant of the plots. This corridor reflected the orientation of the rocker, which only rotated a small amount during each cycle of the mechanism. Because the rocker remained in virtually the same orientation throughout the cycle and because the instant center must lie along a line extending from the rocker, the instant center was confined to that corridor. 


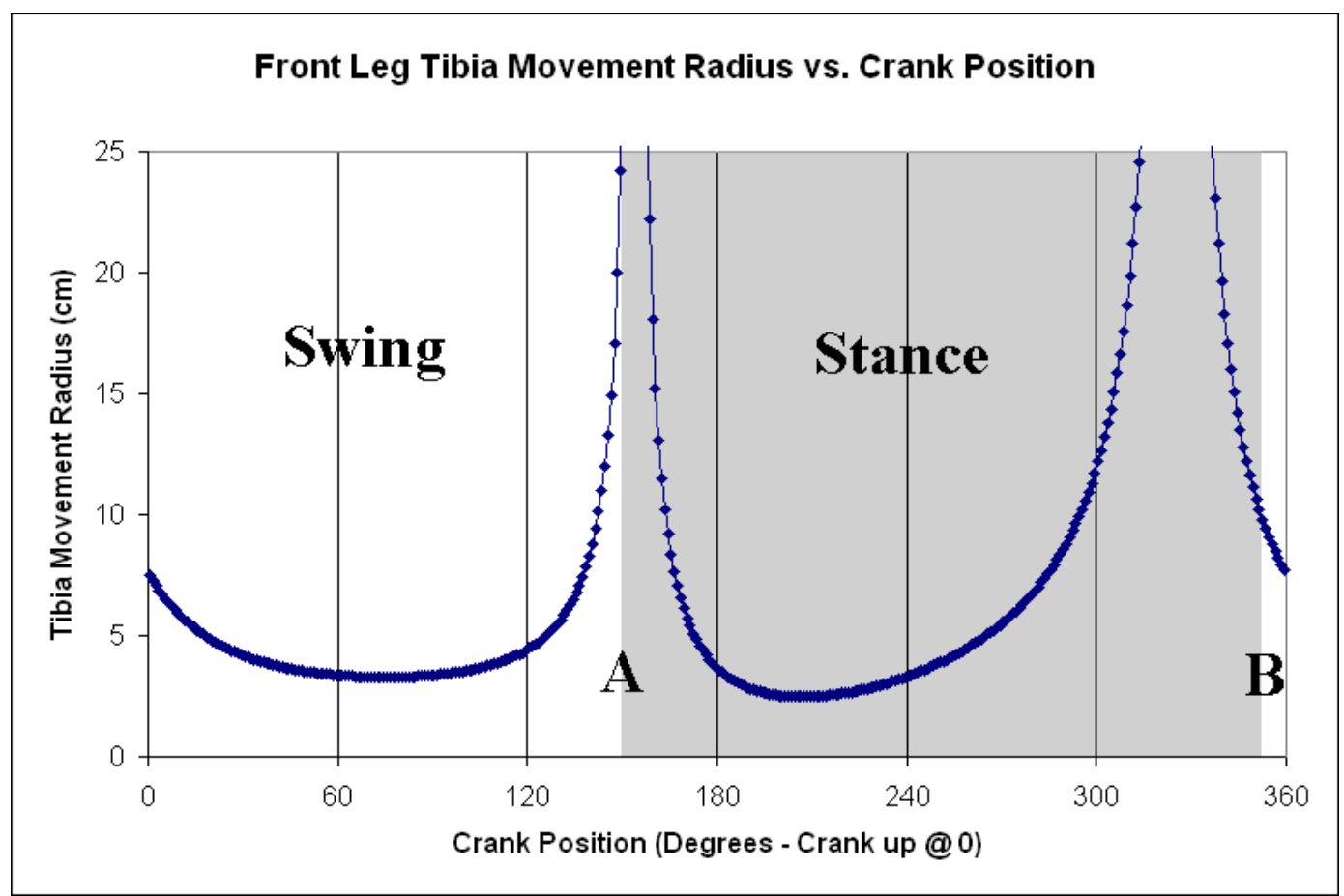

Figure 3-17. Tibia Movement Radius vs. Crank Position

Again, the entire point of calculating the position of the instant center was to calculate the mechanical advantage. In order to make that calculation, the distance from $\mathrm{P}_{1}$ to the instant center was exported from the program and is plotted in Figure 3-17. This distance is referred to as the movement radius, since it is the radius about which the tibia is rotating. This movement radius would be the numerator of the "gear ratio" calculation that is used to determine mechanical advantage.

Unfortunately, when the mechanical advantage was calculated for the first time using this method, it was determined that the instant center location was not going to be a good measure. 


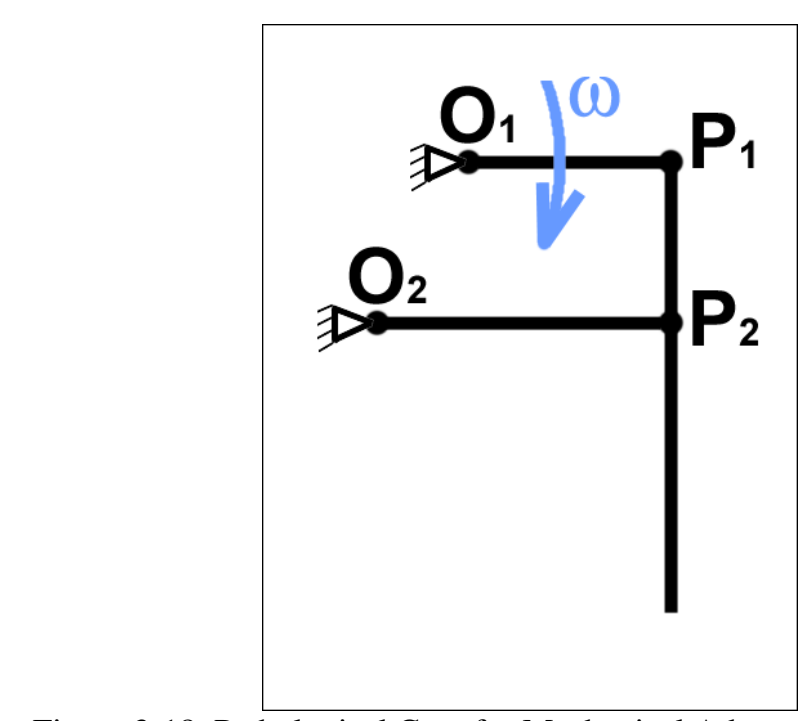

Figure 3-18. Pathological Case for Mechanical Advantage Calculations

To explain why the instant center location was a poor indicator of mechanical advantage, consider the pathological case illustrated in Figure 3-18. As in previous figures, $\mathrm{O}_{1}$ is the location of the driveshaft that is driving the crank. $\mathrm{O}_{2}$ is where the rocker connects to the chassis of the robot. $\mathrm{P}_{1}$ and $\mathrm{P}_{2}$ are joints where the crank and rocker connect to the tibia. For the purpose of simplicity, assume that all angles in the illustration are right angles. In this particular case, if the crank is rotating clockwise, the input force transferred from the crank to the tibia should be equal to the output force seen at the foot, and the mechanical advantage should be equal to one.

However, the crank and the rocker are parallel, and the instant center is therefore located at an infinite distance away. The resulting mechanical advantage calculated from the instant center location could not be equal to one. It was for this reason that the mechanical advantage was calculated using other means.

Recall that the program had already created the vectors defining the various links of the mechanism. Using those vectors and the specified crank velocity $\left(\omega_{1}\right)$, the angular 
velocity of the coupler link $\left(\omega_{2}\right)$ could be found using equation 3.19 of Wilson's Kinematics and Dynamics of Machinery, 2nd Ed [46]:

$$
\omega_{2}=-\omega_{1} \cdot\left(\frac{r_{1 y} \cdot r_{3 x}-r_{3 y} \cdot r_{1 x}}{r_{2 y} \cdot r_{3 x}-r_{3 y} \cdot r_{2 x}}\right)
$$

where $\omega_{1}$ is the angular velocity of the crank, and $r_{1}, r_{2}$, and $r_{3}$ are the vectors that define positions of the crank, coupler, and rocker, respectively. The $\mathrm{x}$ and $\mathrm{y}$ subscripts denote horizontal and vertical components of those vectors.

Once the coupler's angular velocity was found, the velocity components of the foot could be determined using equation 3.21 from the same source [46]:

$$
\begin{aligned}
& V_{f o o t, x}=\dot{P}_{1 x}+\omega_{2} \cdot r_{f o o t, y} \\
& V_{f o o t, y}=\dot{P}_{1 y}-\omega_{2} \cdot r_{f o o t, x}
\end{aligned}
$$

The velocity of the foot was a function of the velocity components of $\mathrm{P}_{1}$, the joint that connects the crank and the coupler. It was also dependent upon the angular velocity of the coupler $\left(\omega_{2}\right)$ and $\mathrm{r}_{\text {foot }}$, the vector that extended from $\mathrm{P}_{1}$ to the foot of the leg.

The velocity ratio between the output point (the foot) and the input point (the crank) was then calculated. This velocity ratio was equal to the mechanical advantage. Because both the input and output velocities were proportional to the angular velocity of the crank $\left(\omega_{1}\right)$, the mechanical advantage should remain the same, no matter the value of the crank velocity. 
These calculations made it possible to plot the mechanical advantage vs. the crank position. These plots can be seen in Section 3.7, where the leg mechanisms finally chosen for use on the robot were analyzed.

\subsubsection{Evaluation of Finished Legs}

Before any parts were machined, everything was modeled in Pro/ENGINEER, which is a software package made by the Parametric Technology Corporation. The software allowed 3D models to be created, complete with all of the features that the actual part would have, including holes for fasteners. Assemblies could be created in which individual parts were assembled the way they would be if the physical parts existed (see Figure 3-19). Software like Pro/ENGINEER is indispensable, because it allows the designer to check that parts are going to mate with other parts they way the designer expected. Unexpected interference with other parts is not something that can be determined otherwise.

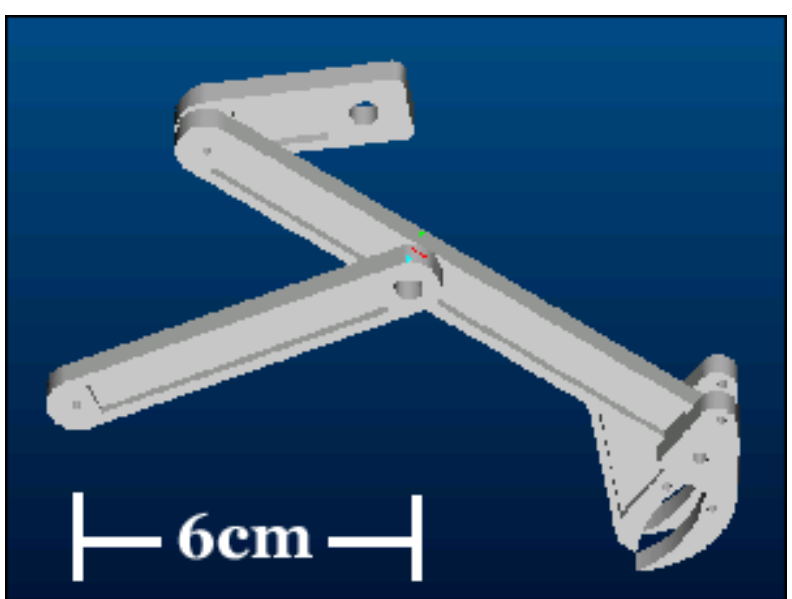

Figure 3-19. Parts and Assemblies Created in Pro/ENGINEER

Pro/ENGINEER was also able to export the part files in a format that could be understood by CNC machines. By the time the legs were actually constructed, a newer, larger CNC milling machine was available for use in the Biologically Inspired Robotics 
Lab than the one used to machine the impellers described in Chapter 7. Most of the parts on MechaRoach II were machined on a Hurco VM1 CNC machine. The ones that were not machined on the Hurco were manually machined on a vertical milling machine or lathe.

MechaRoach II could have been assembled from parts that were all manually machined. However, CNC machines can create parts with curves or other surfaces that are too complex to be machined manually. Therefore, had all of the parts been machined manually, they would have had to be designed differently, and the robot probably would have been heavier as a result.

Before the very first leg was actually built, a small, scaled prototype was constructed from aluminum (see Figure 3-20).

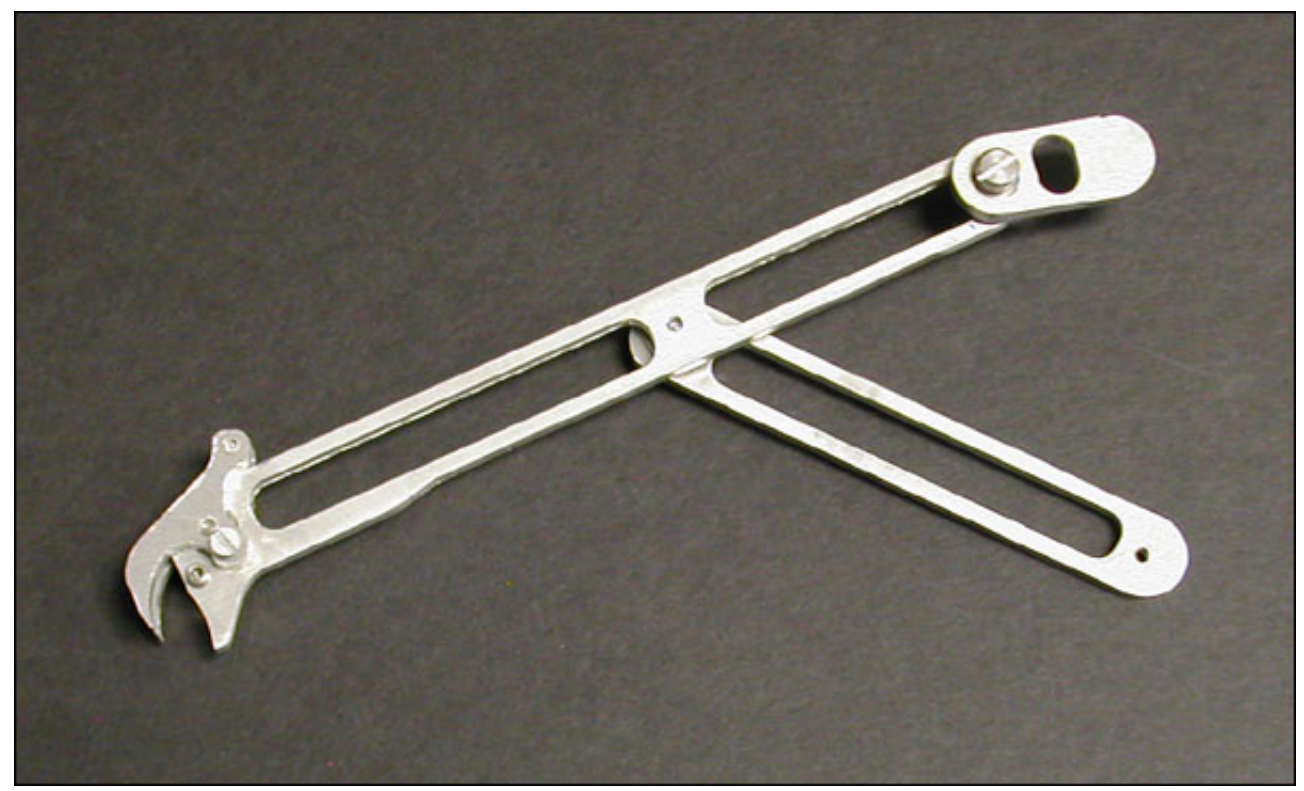

Figure 3-20. A Small, Scaled Prototype of the Front Leg

The prototype was built to test joints and foot designs. It was rudimentary, but construction techniques were improved and small revisions to the leg designs could be made. As a result of building this prototype, changes were made before large amounts of material were devoted to leg construction. 
Originally, the chassis of the robot and the legs were designed together. However, changes needed to be made to the leg designs. By the time new legs were machined for use on the robot, construction of the chassis had been completed, and the legs merely had to be bolted on.

As a first test of the leg mechanisms, MechaRoach II was operated on a level surface to determine whether the legs would power a smooth walking gait. The inter-leg coordination was adjusted to visually determine whether changes improved walking performance and whether changes brought the walking pattern closer to a tripod gait.

If a smooth walking gait had been created, small obstacles were placed in the path of MechaRoach II to determine if the legs were being raised high enough during swing to clear them.

Finally, if the other initial tests were successful, a transition between a horizontal surface and an inclined surface was attempted.

The results of these tests were mostly subjective. As previously stated, there were many tradeoffs to be made in the design process. And often, these tradeoffs had to be revisited to determine if the optimal mechanism design had been reached. The design process described in this section was applied iteratively to incrementally improve the performance of the legs until they exhibited the desired behavior.

For instance, if the new leg dragged its foot during swing, the mechanism design was adjusted in Working Model so that the leg would raise its foot higher. The adjusted mechanism was then reanalyzed with the Mechanism Analyzer program, and then the new version of the leg was built, and the process was repeated. Unfortunately, even the smallest changes often required most of the parts of the leg to be remachined. The 
process consumed large amounts of time and material despite all attempts to conserve them.

\subsection{Poor Mechanism Design Example}

\subsubsection{The Mechanism}

Figure 3-21 shows an example of a poor 4-bar mechanism to be used as a robot leg. It was investigated for use as a middle leg. The components of the mechanism are blue, the chassis is not shown, and the figure is a screenshot from the Working Model software package from Knowledge Revolution.

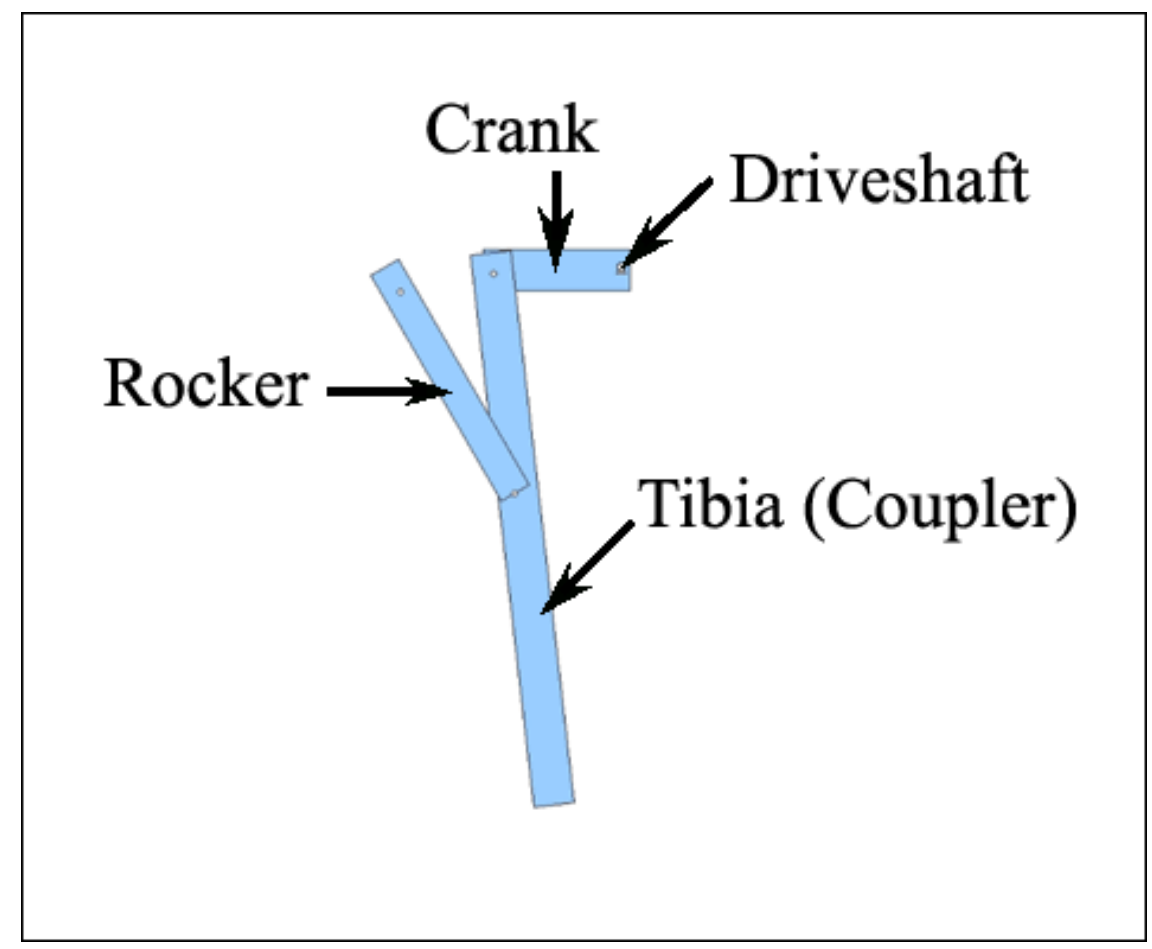

Figure 3-21. Poor 4-Bar Mechanism Leg Example

The elements of this example are the same as the elements of the 4-bar mechanisms that were used on the robot and are analyzed in later chapters. There is a crank, which is driven by a driveshaft at one end. The mechanisms are designed to be powered by a continuously rotating driveshaft that is spinning at a constant speed. At the other end, the 
crank makes a connection to the tibia through a passive pin joint. The rocker link, as previously explained, makes a connection on one end with the chassis and with the tibia at the other end. Both connections are passive pin joints. The free end of the tibia link is treated as the foot of the leg.

The data in Figures 3-22, 3-23, and 3-24 were exported by the program that is described in Section 3.5.4. The program takes the geometry of a 4-bar mechanism, assumes the crank of the mechanism to be rotating at $1 \mathrm{rad} / \mathrm{sec}$, and then exports the resulting footpath, mechanical advantage curves, and other dynamic data that can be used to analyze the mechanism. One data point was exported per degree of rotation of the crank (360 total). The mechanisms complete one swing-stance cycle for every 360degree rotation of the crank. The exported data was then plotted in Microsoft Excel.

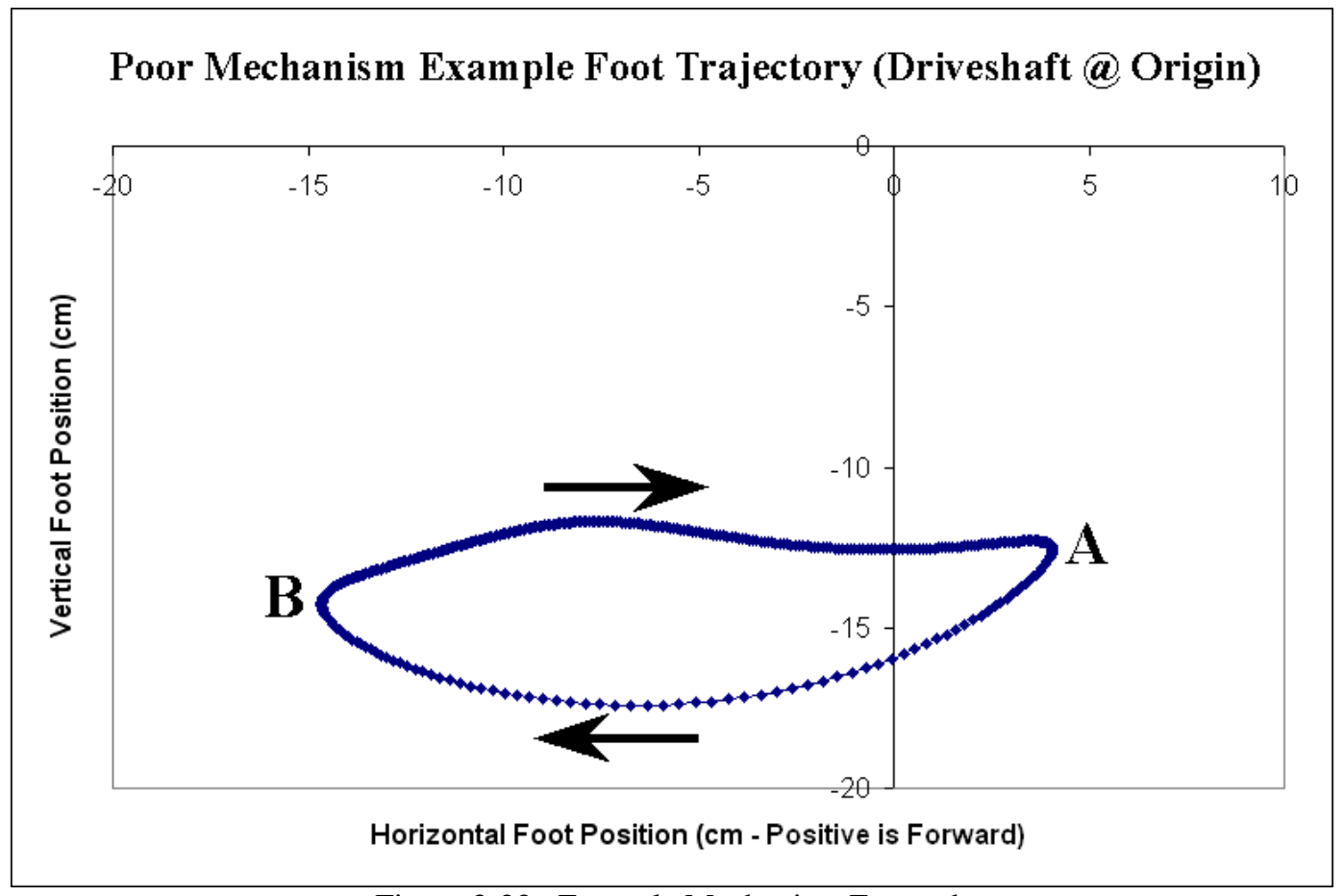

Figure 3-22. Example Mechanism Footpath

Figure 3-22 shows the footpath of the example mechanism shown in Figure 3-21. When the robot is moving forward, it is moving along the positive $\mathrm{x}$-axis. The driveshaft 
of the mechanism is located at the origin of the coordinate system. All of the driveshafts in the robot lie in the coronal plane.

The foot moves along the path in the clockwise direction. At Point A, the leg makes the transition from swing to stance, and at Point B, the leg makes the transition back to swing.

\subsubsection{Positive Characteristics}

As stated previously, the cockroach stride lengths are about $25 \%$ of the total body length. Given a robot length of $66 \mathrm{~cm}$, the stride produced by this mechanism is about the right length.

The vertical displacement during stance is also relatively small, which is a positive characteristic. Ideally, the trajectory of the foot during stance should be as horizontal as possible, but a small amount of deviation is acceptable.

Other good characteristics of this mechanism are the locations where heel strike and toe off occur. As previously stated, middle legs should begin stance by creating decelerating forces in the longitudinal direction, and then transition to creating accelerating forces by toe off. This suggests that heel strike should occur with the foot in front of the shoulder (or driveshaft), which is an orientation in which creating decelerating forces is easier. Toe off should occur with the foot behind the shoulder (or driveshaft), which is an orientation in which creating accelerating forces is easier.

Finally, the linkage mounting locations are in acceptable locations. The location where the rocker connects to the chassis is near the driveshaft, and construction should therefore be possible. 


\subsubsection{Negative Characteristics}

Unfortunately, the mechanism has several poor characteristics, which makes it unsuitable for use as a leg on MechaRoach II.

The data points in Figure 3-22 are separated by larger distances during stance, compared to the swing portion. The wider separation between data points in stance indicates that the foot velocity is higher during that phase. This conclusion is confirmed by the data in Figure 3-23, which shows magnitudes far higher during stance (grey area) than during swing (white area).

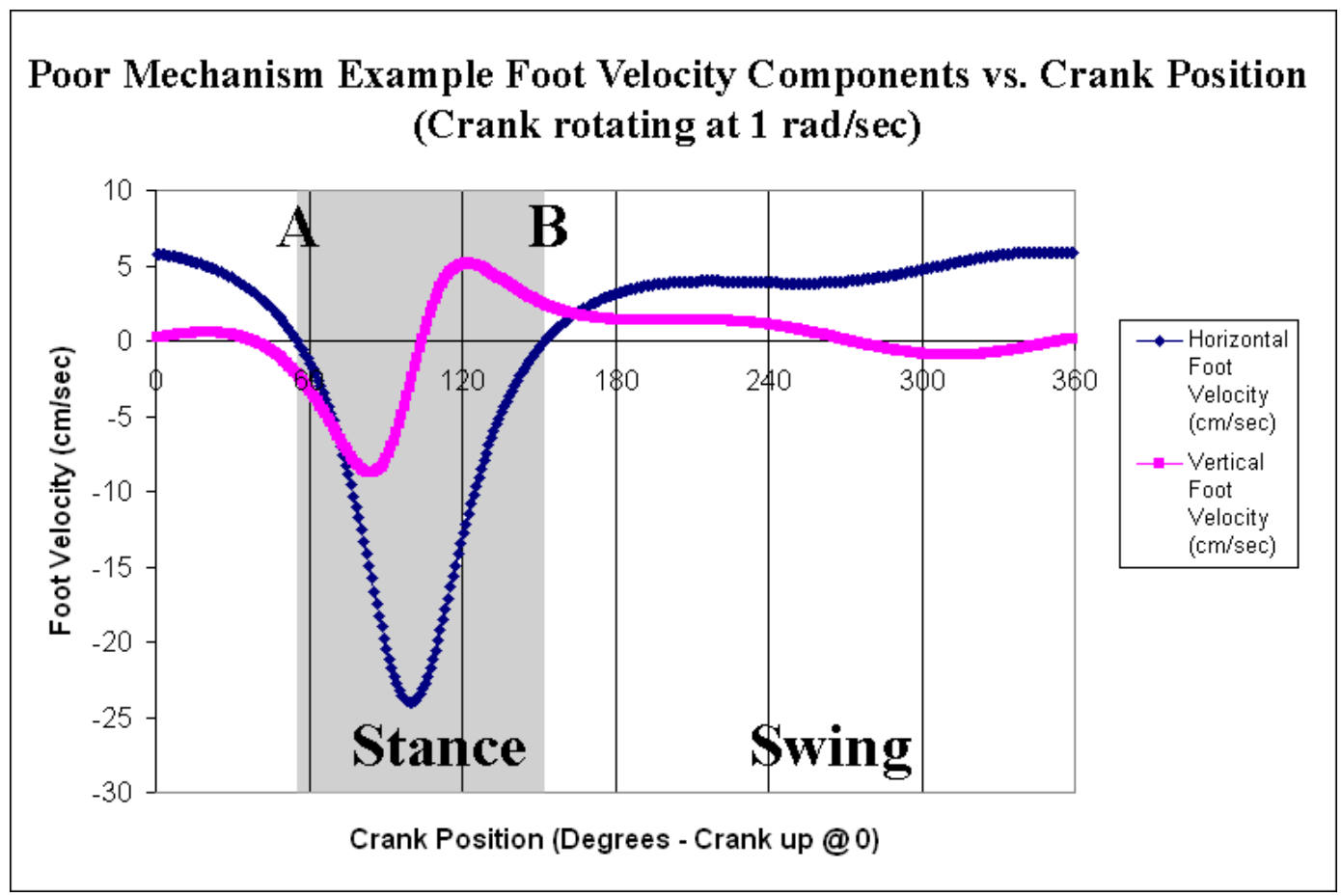

Figure 3-23. Example Mechanism Foot Velocity Components

The convention is the same between Figures 3-22 and 3-23. The leg transitions from swing to stance at Point A, and in the opposite direction at Point B. When the foot moves forward with respect to the chassis, it is in the positive-x direction. And when the foot is raised up, it is moving in the positive-y direction. 
The high velocity components during stance are undesirable for a number of reasons. Ideally, the leg would spend the majority of the time in stance supporting the robot, and a minimal amount of time in swing, when it cannot support the robot. However, this mechanism spends the majority of its time (about 75\%) in swing. If all of the legs spent that percentage of their time in swing and all of their cranks were rotating at a constant velocity, there would be no overlap between the supporting phases of the two tripods in an alternating tripod gait. As a result, there would be periods of time when all of the legs were being swung forward. The legs would make contact with the ground during swing, and the robot would be propelled backward, which is undesirable.

The high velocity components also result in poor mechanical advantage values during stance, when the leg is actually loaded (see Figure 3-24).

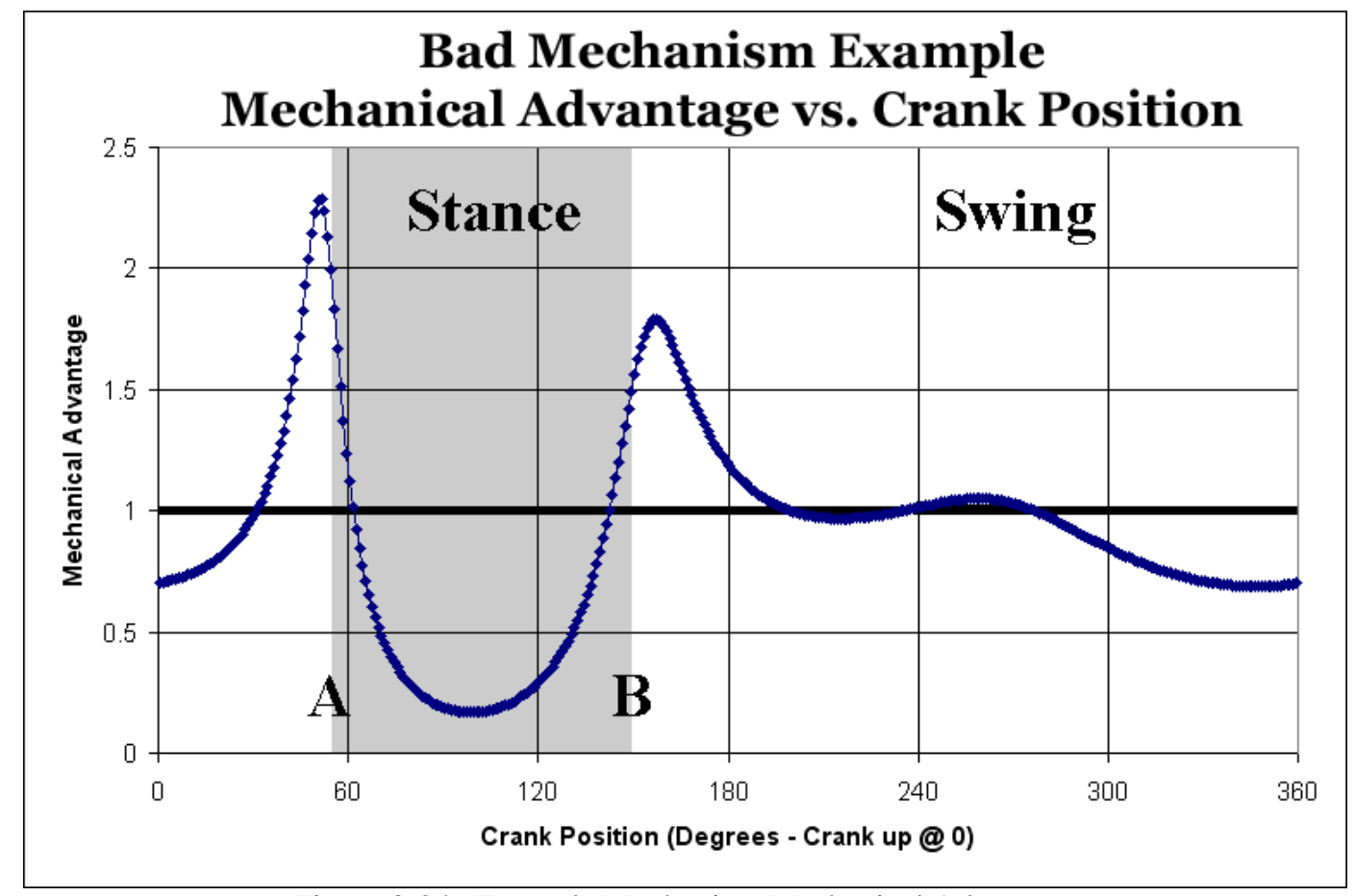

Figure 3-24. Example Mechanism Mechanical Advantage

A mechanical advantage of 1 would mean that the force output of the mechanism at the foot is equal to the input force by the driveshaft. Values below 1 mean that the output 
force is lower than the input force. Gear reductions in the drivetrain allow legs to produce sufficient thrust forces even with mechanical advantage values below 1 during stance. However, this mechanism only has a low mechanical advantage during stance. Ideally, the mechanical advantage would be higher during stance, and lower during swing, when the leg is not needed for support or propulsion.

High foot velocities during stance also result in large changes in horizontal velocity. During stance, for the purposes of leg coordination, the horizontal velocity should be as constant as possible. Unfortunately, this mechanism has nearly a constant horizontal velocity during swing, and the largest changes during stance. During swing, it is neither required nor desired to have a constant horizontal velocity.

The final undesirable characteristic of this mechanism is the leg length. In order to achieve the appropriate stride length, the tibia length had to be relatively long. As a result, in the middle of stance, the driveshaft of the robot will be $17 \mathrm{~cm}$ above the ground. This is about twice the desired height. As previously explained, the distance of the center of gravity of the robot to the substrate is directly proportional to the attachment force that would be required to keep the robot on a vertical surface. The mechanisms that were eventually chosen for use as legs on MechaRoach II all created a proportionately smaller stride lengths for this reason. Shorter tibias would allow the center of gravity to be kept closer to the substrate. However, shorter tibias would also result in a lower walking speed and smaller ground clearance, which negatively impact the robot's obstacle climbing ability. This was one of the many tradeoffs that had to be made during the design process. 


\subsection{Final Leg Designs}

The 4-bar mechanisms that were used as legs were implementations of abstracted cockroach footpaths. Although the major features of the cockroach footpaths were supposed to be recreated by the mechanisms, there were significant deviations.

The footpaths of the cockroach walking on a treadmill are shown in Figure 3-25. The reference frame for the figure is the ground, not the body of the animal.

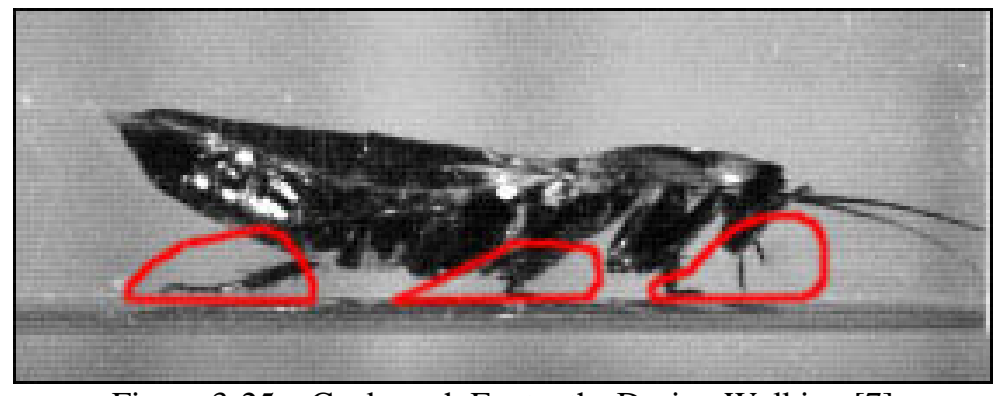

Figure 3-25. Cockroach Footpaths During Walking [7]

As previously stated, the front legs of the animal are raised high so that obstacles can be surmounted without active changes in trajectory [44]. The front legs produce decelerating forces, the middle legs produce decelerating forces at the beginning of stance and accelerating forces at the end, and the rear legs create accelerating loads in the longitudinal direction [12].

\subsubsection{Leg Size}

The Blaberus disoidalis' body is typically $4.41 \mathrm{~cm}$ long [21]. Since the robot's body is $66 \mathrm{~cm}$ long, the robot is about fifteen times larger than the cockroach.

From the body-coxa joint to the end of the tarsus, a cockroach's front leg is about $2.4 \mathrm{~cm}$, the middle leg is about $2.9 \mathrm{~cm}$, and the rear leg is about $3.7 \mathrm{~cm}$ [21]. Scaled up fifteen times, the front leg should be $36.6 \mathrm{~cm}$, the middle leg $44.9 \mathrm{~cm}$, and the rear leg $56.3 \mathrm{~cm}$. Unfortunately, due to mechanical advantage constraints, the legs on the robot 
were required to be proportionately smaller. The length of the front leg coupler was $15.5 \mathrm{~cm}$, the middle leg coupler was $17 \mathrm{~cm}$ long, and the rear coupler was $19 \mathrm{~cm}$ long. The resulting stride lengths are also proportionately shorter, as compared to the cockroach's.

\subsubsection{MechaRoach II’s Front Legs}

The front leg design that was finally chosen for the robot is shown in Figure 3-26. The right front leg is shown, and the left front leg can be seen in the background.

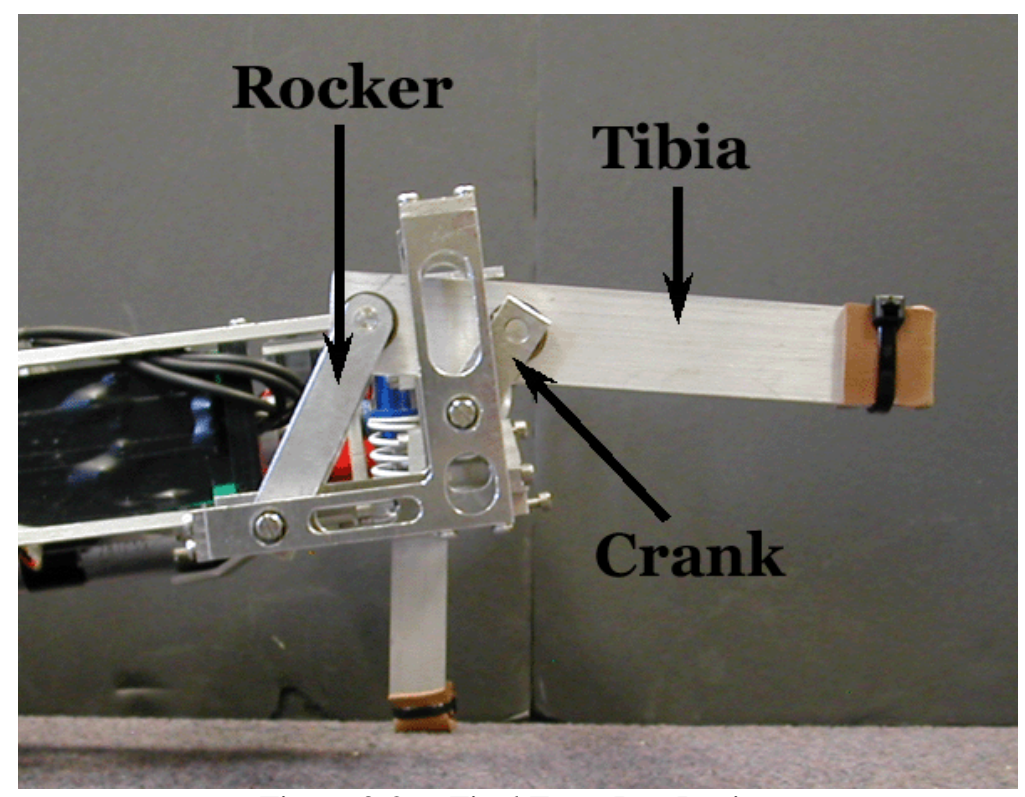

Figure 3-26. Final Front Leg Design

All of the following plots of foot trajectory, foot velocity, and mechanical advantage are derived from data exported by the Mechanism Analyzer program described in Section 3.5.4. 


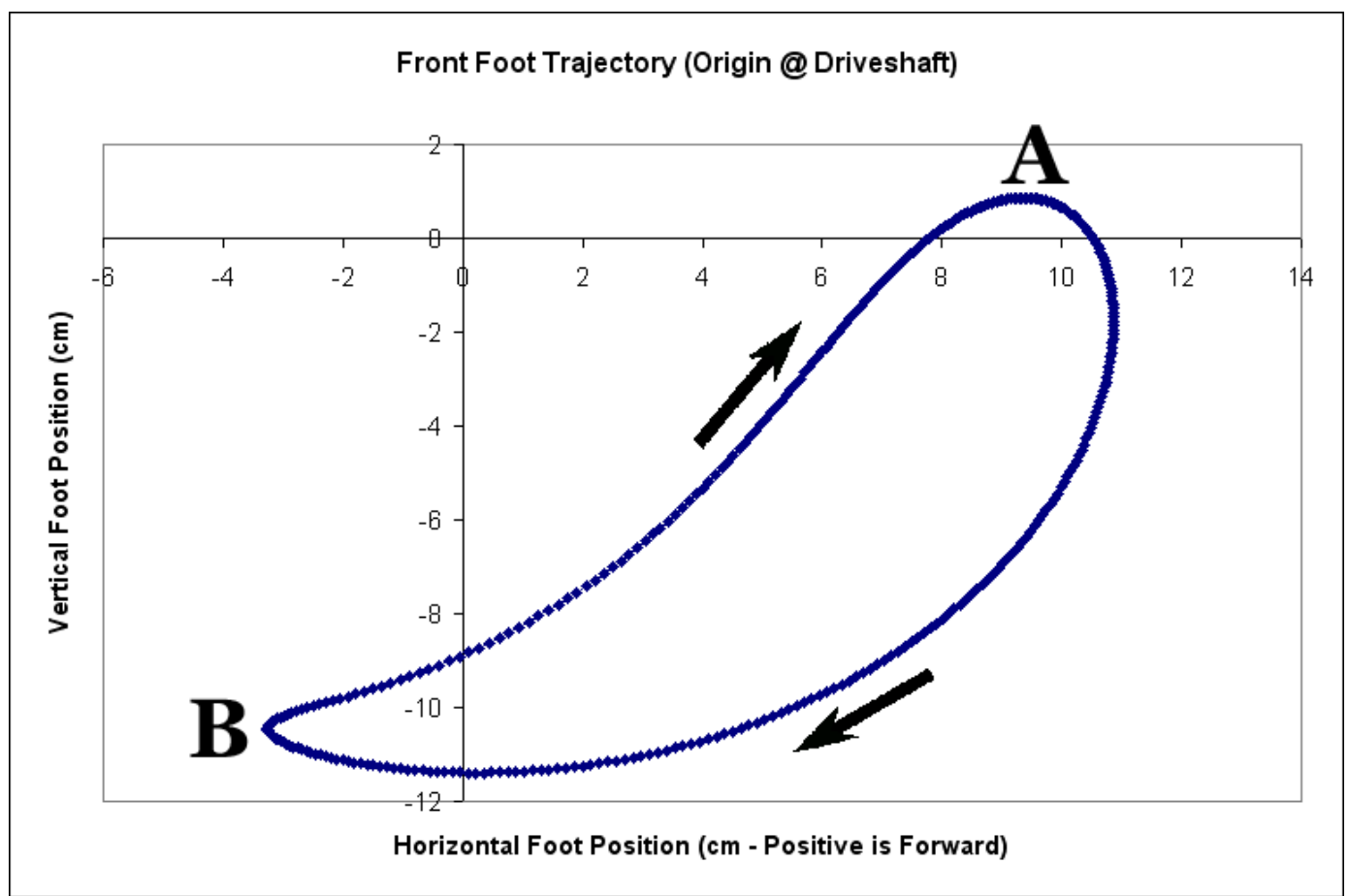

Figure 3-27. MechaRoach II’s Front Leg Footpath

The robot's front leg footpath is shown in Figure 3-27. The front of the robot lies along the positive-x axis, and the driveshaft that powers the mechanism lies at the origin of the coordinate system. All of the driveshafts in the robot lie in the coronal plane. The plane that the footpath lies in is parallel to the sagittal plane of the robot.

As shown in the figure, the front foot is raised above the level of the driveshaft during swing. The foot is then drawn downward, to allow obstacles to be surmounted. After the foot is swept downward, the foot is drawn back during the rest of the stance phase. The trajectory in Figure 3-27 looks very different than the cockroach front leg trajectory in 325. However, the cockroach was walking on a treadmill, and was viewed in the reference frame of the ground. In contrast, the reference frame for the robot's foot trajectory is fixed to the chassis. Therefore, the two figures are not depicting the same information and are not comparable. 
Point A denotes where the trajectory transitioned from swing into stance. Although heel strike would not occur at Point A during normal walking, it might if the leg encountered an obstacle. Therefore, Point A is placed at the earliest time in the trajectory where the front leg could start supporting the load of the robot, and it is defined as the location where the trajectory changes from having a positive vertical velocity to a negative one (see Figure 3-29).

Similarly, Point B denotes where the trajectory transitioned from stance into swing. It is defined as the location where the trajectory changes from having a negative horizontal velocity to a positive one.

The stride length is approximately $14 \mathrm{~cm}$, which was $21 \%$ of the body length. That proportion of stride length to body length is near the cockroach's. Unfortunately, heel strike did not occur during normal walking near point A. During normal walking, the stride length was expected to be about $8 \mathrm{~cm}$ (12\% of body length), which is roughly half the properly scaled-up cockroach stride length.

The foot reaches $11.38 \mathrm{~cm}$ below the driveshaft during stance.

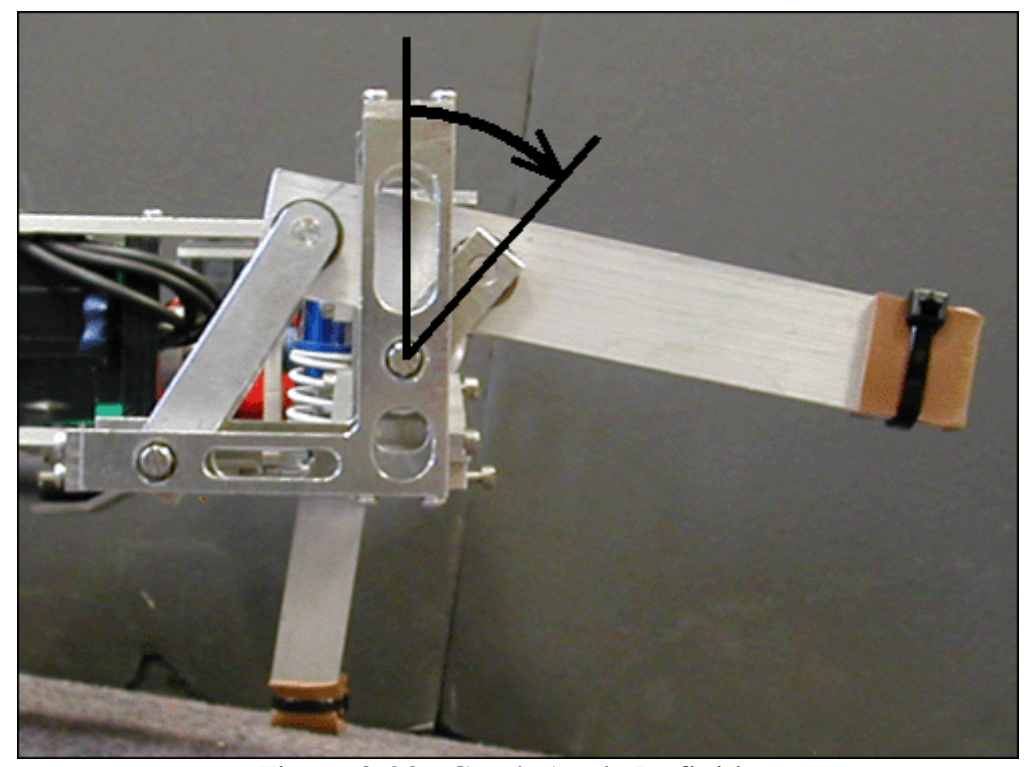

Figure 3-28. Crank Angle Definition 
The way the crank angle was defined is shown in Figure 3-28. The reference frame is oriented the same way as in the footpath plot in Figure 3-27, with the front of the robot lying toward the right. The crank rotates clockwise, and the angle is measured from when the crank was in the vertical position with respect to the chassis, pointing up.

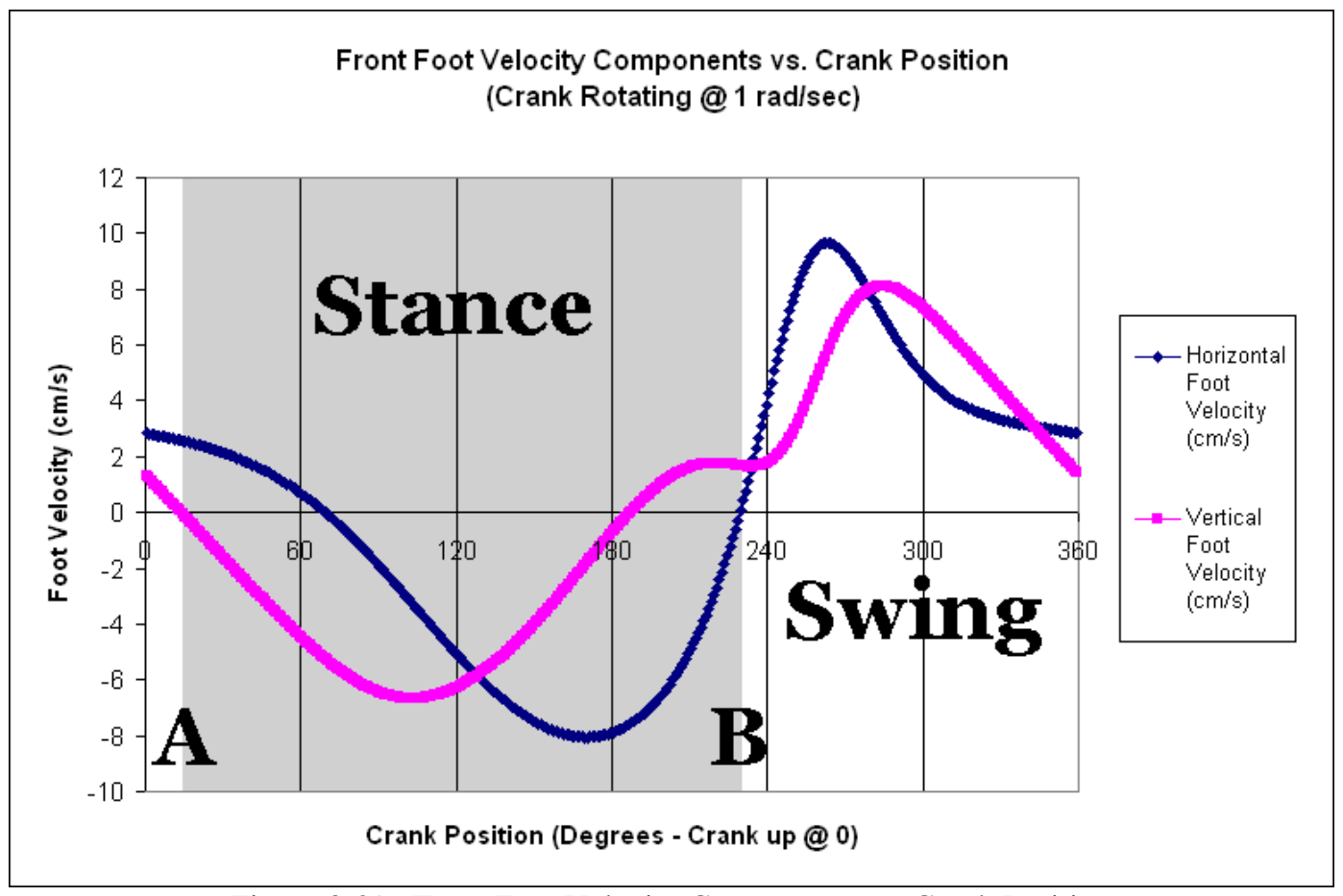

Figure 3-29. Front Foot Velocity Components vs. Crank Position

The components of the foot velocity of the front leg were plotted against the crank angle in Figure 3-29, assuming a constant crank velocity of $1 \mathrm{rad} / \mathrm{sec}$. $1 \mathrm{rad} / \mathrm{sec}$ was chosen because it was the expected crank velocity on the robot. Had a higher speed been chosen, the foot velocity plots would have the same shape, but would have proportionately larger magnitudes.

The area with the gray background defines the portion of the linkage’s cycle when the leg can be in stance. The rest of the area defines when the linkage is in swing. The leg completes its stance-swing cycle with every full rotation of the crank, and only $40.3 \%$ of the cycle is spent devoted to swing if the crank is rotating at a constant velocity. 
As expected from the footpath diagram, the foot velocity is generally downward and rearward during stance, and forward and upward during swing. The velocity magnitudes reach maximum values during swing, and have lower absolute maximums during stance.

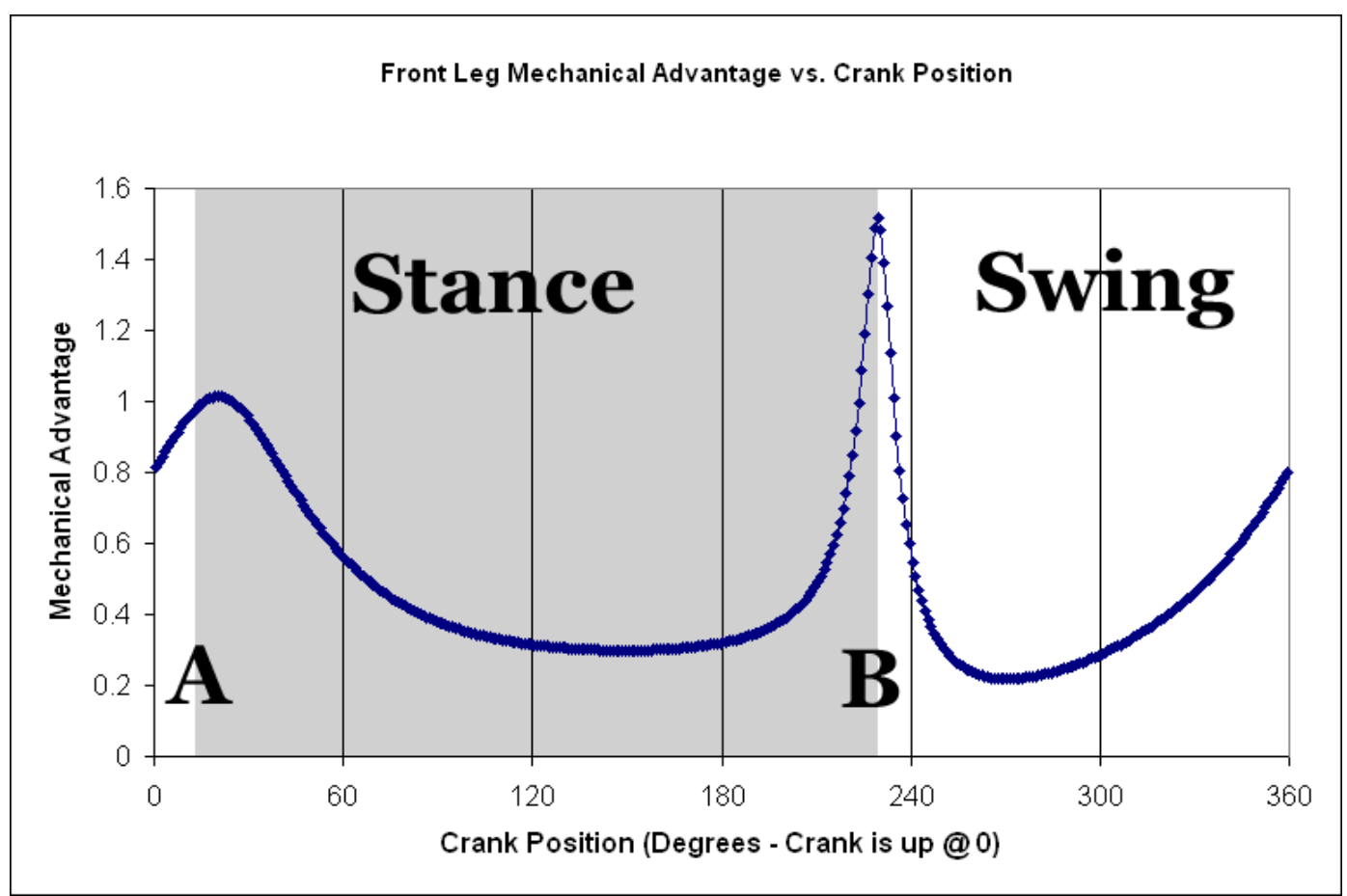

Figure 3-30. Front Leg Mechanical Advantage vs. Crank Position

The leg's mechanical advantage was plotted against crank position in Figure 3-30. Mechanical advantage is the ratio between the output force and input force. The mechanical advantage drops to 0.298 during stance, meaning that the output force at the foot is only $29.8 \%$ of the input force by the crank. The mechanical advantage drops down to 0.219 during swing, which is acceptable because the leg is not loaded during that phase.

The mechanical advantage reaches local maximums during the transition between stance and swing. During the transitions, the mechanism has to switch between moving the foot downward and rearward and moving the foot upward and forward. While the 
foot changed directions, the foot velocity is lower, causing the larger mechanical advantage values.

\subsubsection{MechaRoach II’s Middle Legs}

The middle leg design that was finally chosen for the robot is shown in Figure 3-31. The right middle leg is shown in the figure, with the left middle leg in the background.

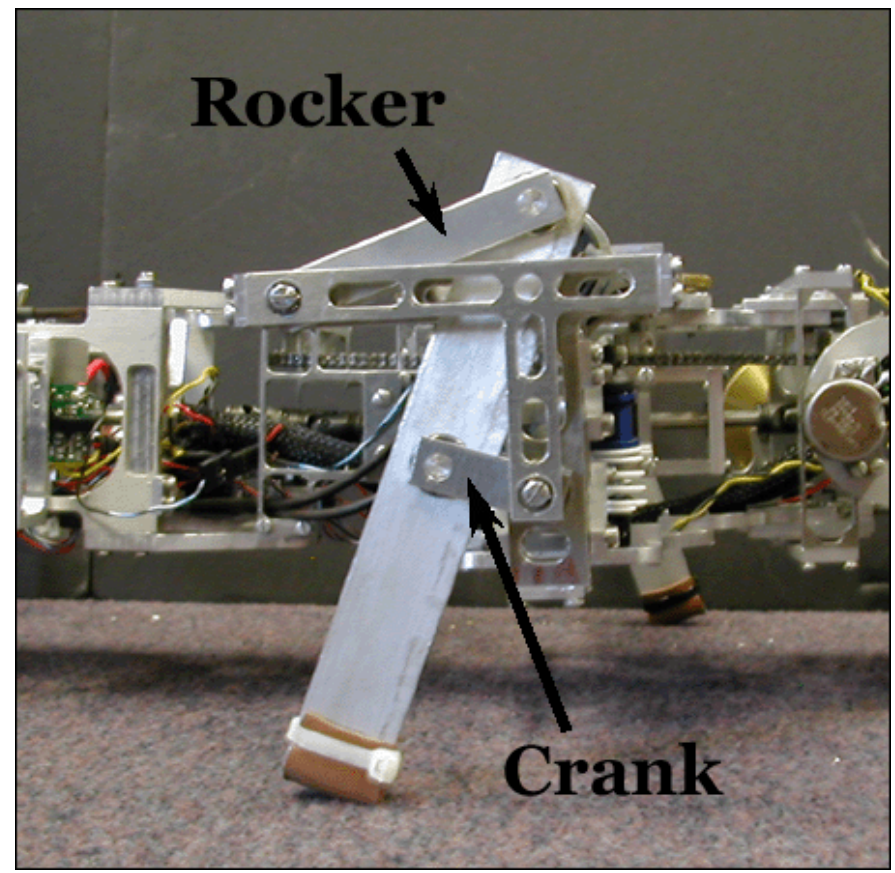

Figure 3-31. Final Middle Leg Design

The foot trajectory of the middle leg is shown in Figure 3-32. The convention is the same as with the front leg footpath. 


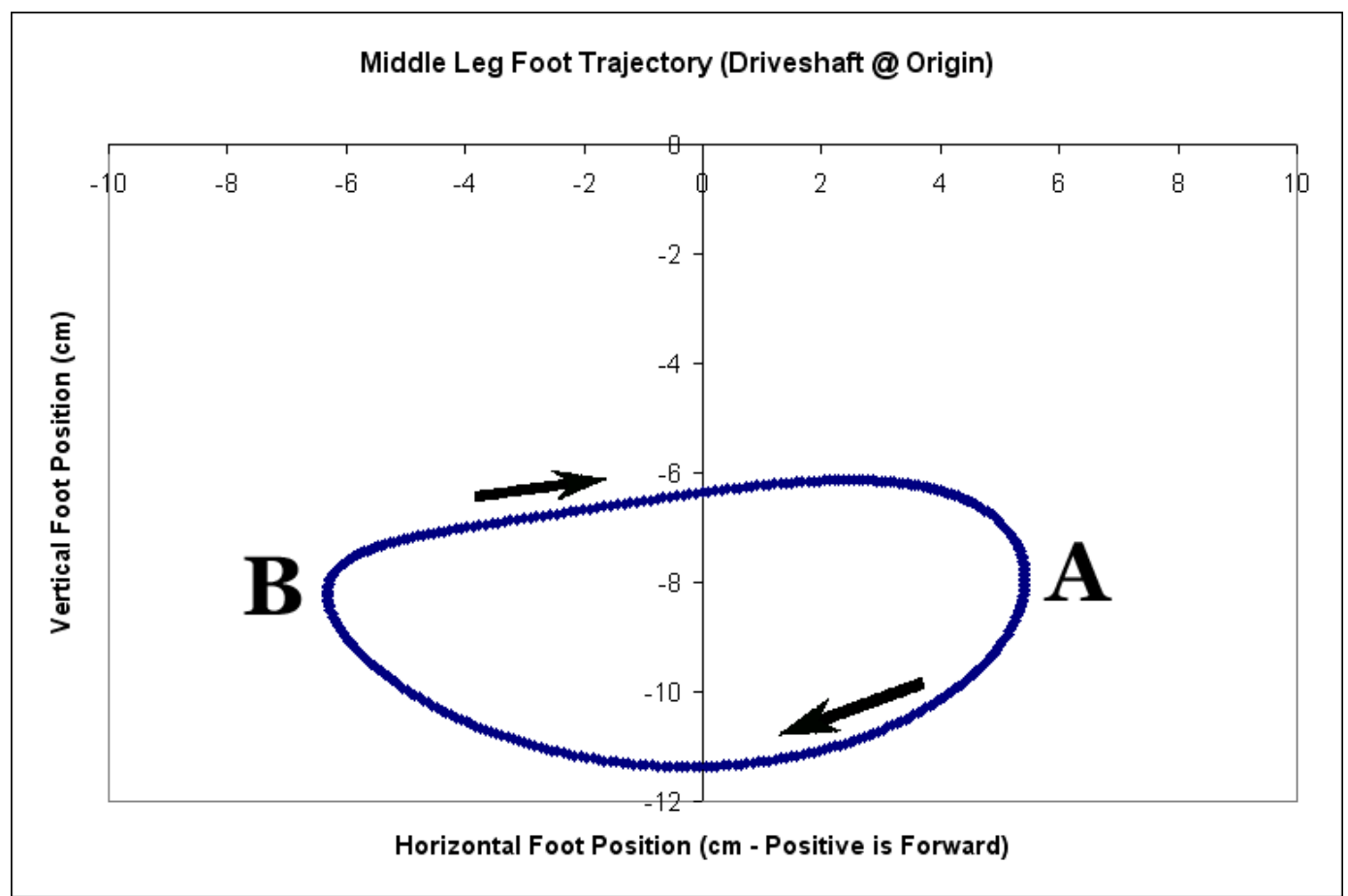

Figure 3-32. MechaRoach II’s Middle Leg Footpath

Again, the footpath of the robot's leg looks different than the trace shown of the cockroach’s middle foot in Figure 3-25, because it has a different reference frame.

Just as in the plots of the front leg, Point A denotes where the leg transitions from swing into stance, and Point B denotes the opposite transition. The transitions between swing and stance are defined as the locations where the horizontal velocity changes between positive and negative values (see Figure 3-33).

Heel strike occurs with the foot ahead of the driveshaft, which puts the leg in a decelerating orientation. The leg then rotates during stance into an accelerating orientation with the foot behind the driveshaft.

The stride length was predicted to be about $11 \mathrm{~cm}$ long (17\% of body length). This stride length is longer than the front leg's, but is still only about $66 \%$ of the properly scaled-up cockroach stride length. 
The foot reaches $11.35 \mathrm{~cm}$ below the driveshaft during stance, which is similar to the front leg.

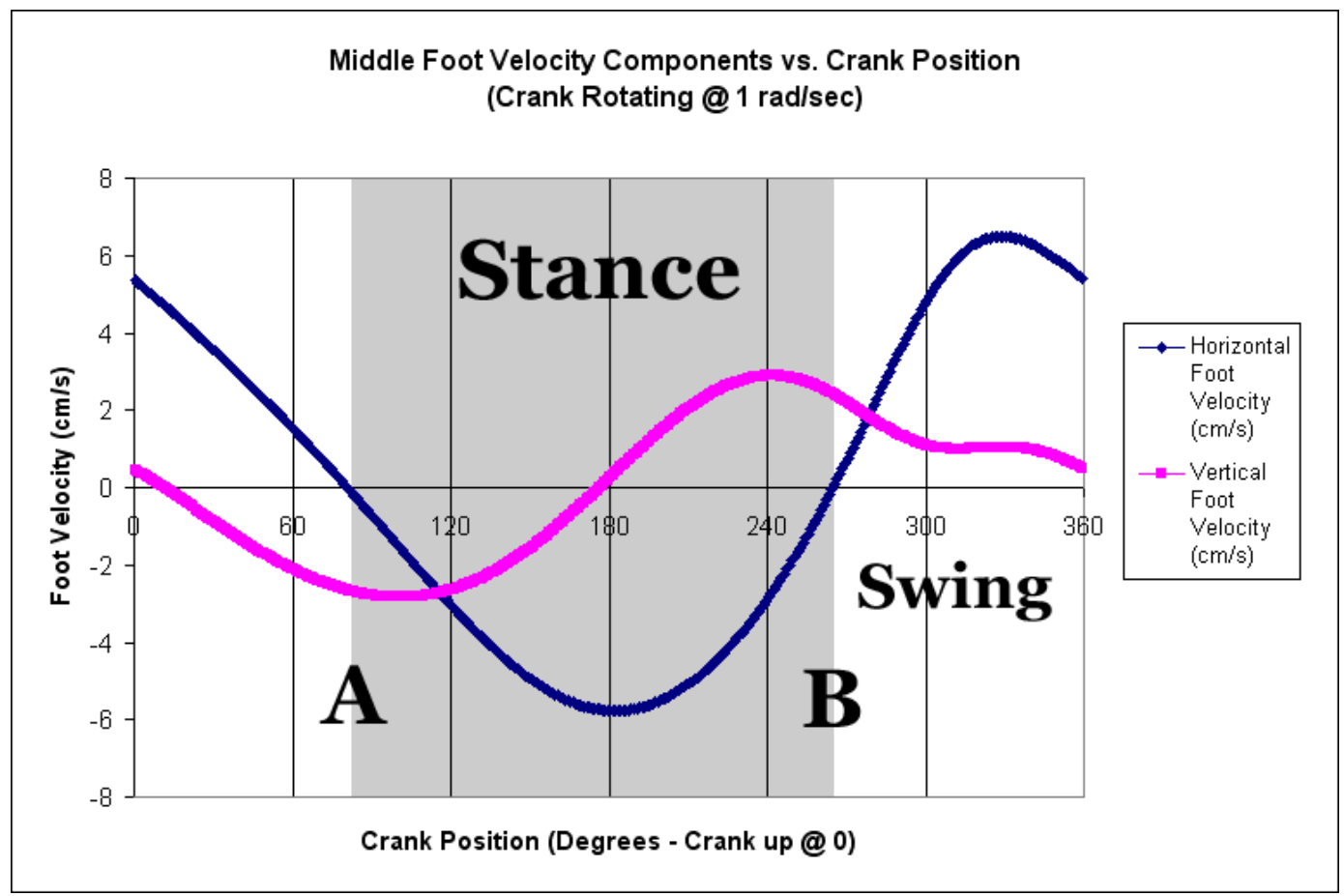

Figure 3-33. Middle Foot Velocity Components vs. Crank Position

The velocity components of the middle foot have been plotted against crank position in Figure 3-33, assuming a constant crank velocity of $1 \mathrm{rad} / \mathrm{sec}$. As with previous plots, the gray area shows where the leg is in stance, and the leg is in swing in the rest of the area. The plot shows one entire swing-stance cycle, and one swing-stance cycle occurs with every full rotation of the crank. $48.9 \%$ of the cycle was devoted to swing. 


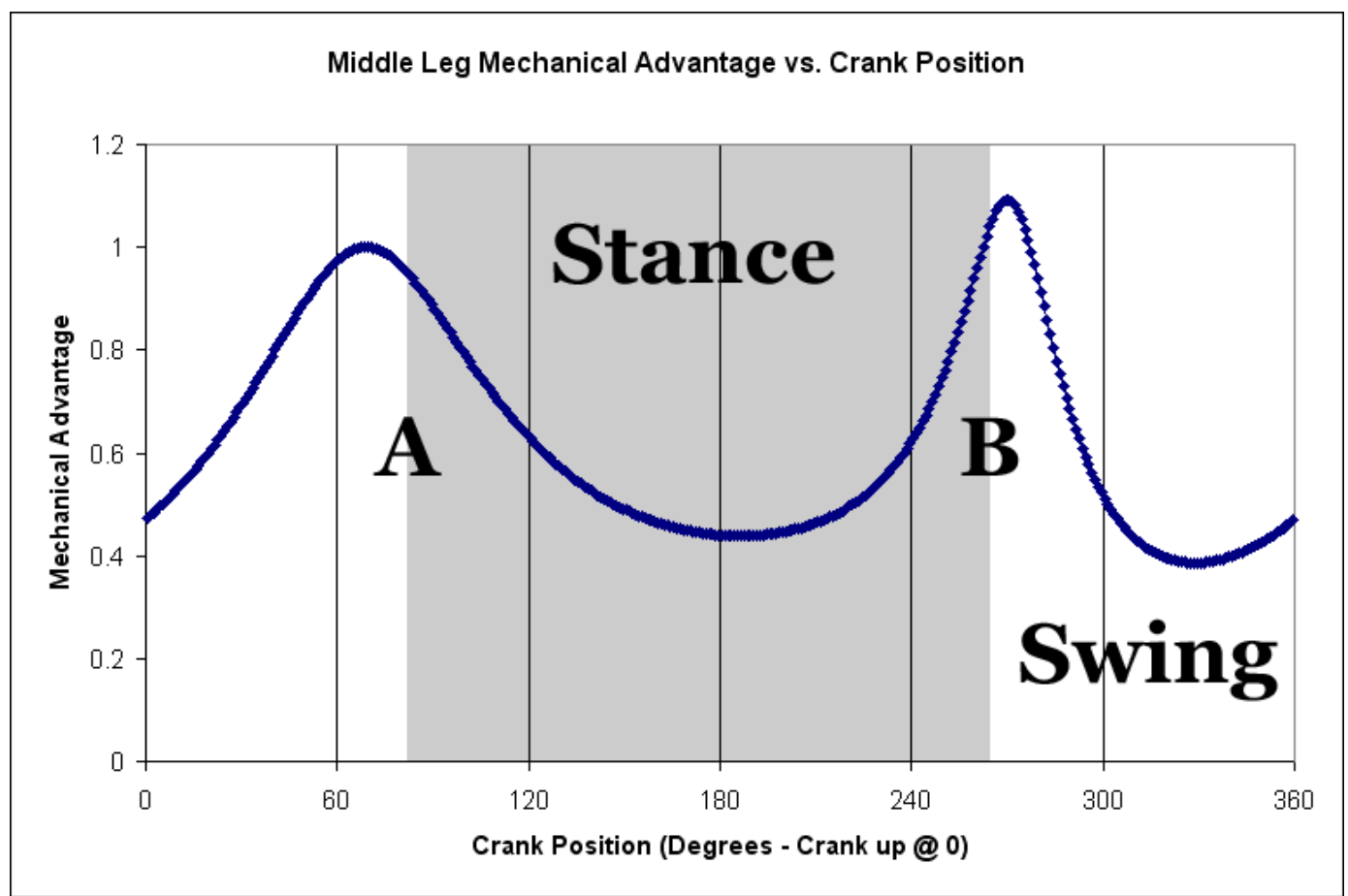

Figure 3-34. Middle Leg Mechanical Advantage vs. Crank Position

In Figure 3-34, the middle leg's mechanical advantage is plotted against the crank position. During stance, the mechanical advantage drops down to 0.439 , and drops even lower during swing (0.389). Similarly to the front leg, the mechanical advantage is higher during the transitions between swing and stance because as the foot changes direction, the overall velocity is lower.

\subsubsection{MechaRoach II's Rear Legs}

The rear leg design that was finally chosen for the robot is shown in Figure 3-35. The right rear leg is shown, with the left rear leg in the background. 


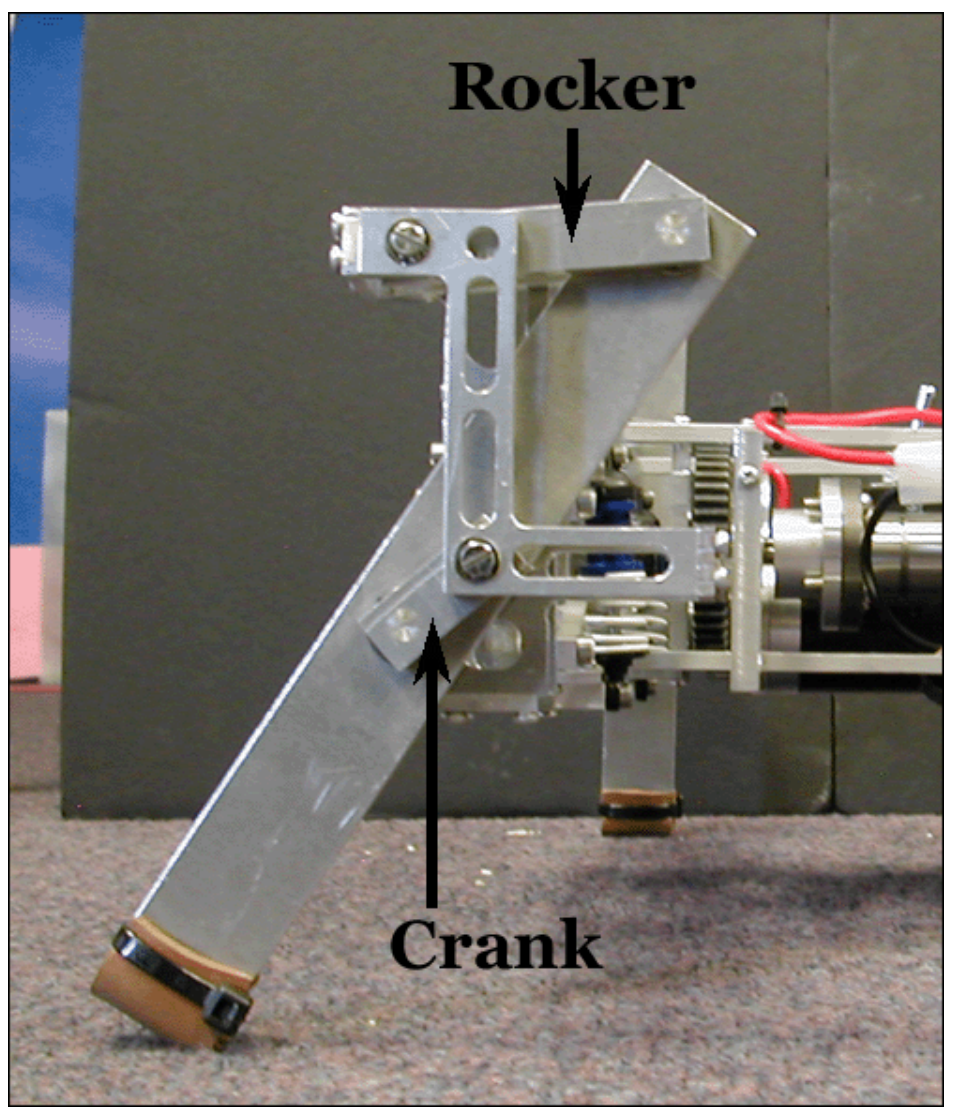

Figure 3-35. Final Rear Leg Design

The footpath of the rear leg of the robot is plotted in Figure 3-36.

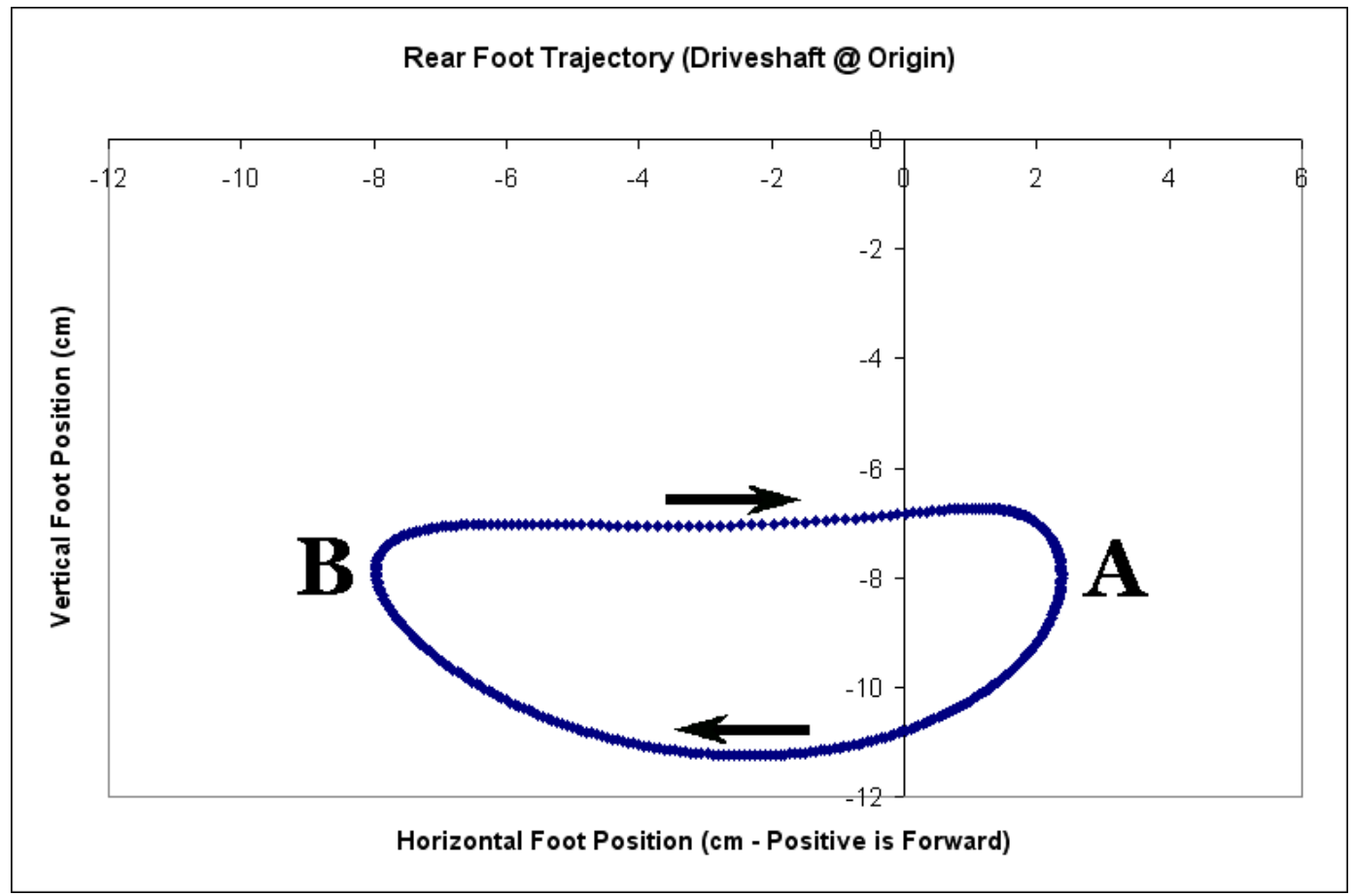

Figure 3-36. MechaRoach II’s Rear Leg Footpath 
The convention of the figure is the same as with previous figures, where the origin of the coordinate system is located at the driveshaft. All of the driveshafts in the robot lie in the coronal plane. The front of the robot is located along the positive-x axis, and the footpath lay in a plane that is parallel to the sagittal plane of the robot.

Point $\mathrm{A}$ is where the leg makes the transition from swing to stance, and Point B is the opposite transition. The transition points are defined as the locations where the horizontal foot velocity changes between positive and negative values (see Figure 3-37).

Heel strike occurs with the foot ahead of the driveshaft, which is a decelerating orientation. However, the majority of stance is spent in an accelerating orientation, so that the rear leg is positioned to provide thrust. Ideally, the rear leg would begin stance in an accelerating orientation, but tradeoffs were made in order to increase the proportion of the leg's cycle devoted to stance. A longer stance portion of the cycle means that the leg can spend more of its time supporting the weight of the robot, and less time in swing. Tradeoffs were also made to maintain a relatively high mechanical advantage during stance so that larger longitudinal forces could be generated with these legs.

The stride length was predicted to be about $10 \mathrm{~cm}$ (15\% of body length). The cockroach's stride length is about $25 \%$ of its body length, making the robot's about $60 \%$ of the properly scaled-up length.

The rear foot reaches a maximum displacement of $11.25 \mathrm{~cm}$ below the driveshaft during stance, which is very close to the displacements seen in the front and middle legs. 


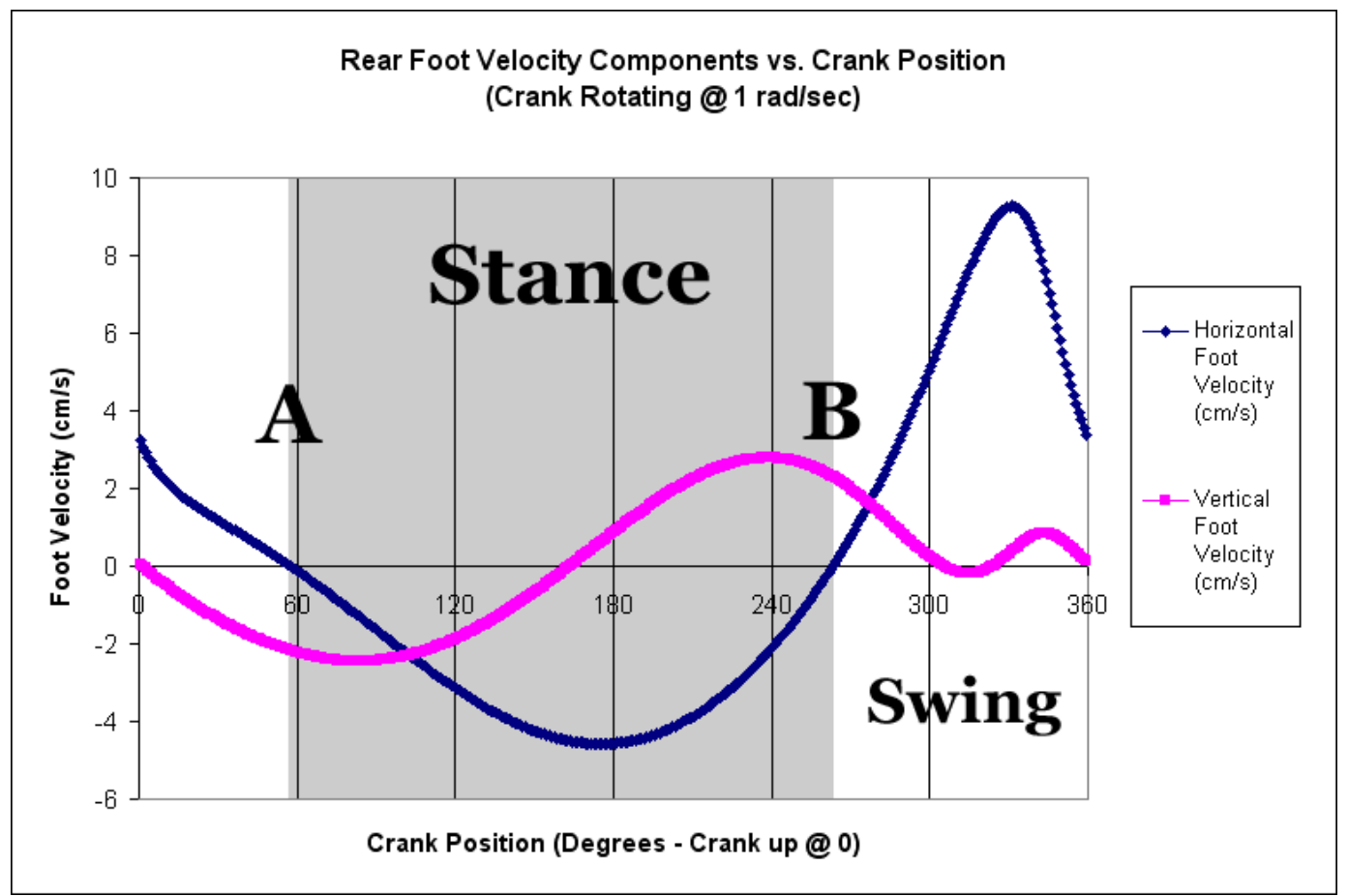

Figure 3-37. Rear Foot Velocity Components vs. Crank Position

The horizontal and vertical foot velocity components were plotted against crank position in Figure 3-37, assuming a constant crank velocity of $1 \mathrm{rad} / \mathrm{sec}$. The gray area of the figure denotes when the leg is in stance, and the rest of the area is where the leg is in swing. The leg makes one stance-swing cycle during one full rotation of the crank, and $43.1 \%$ of the cycle is devoted to swing. 


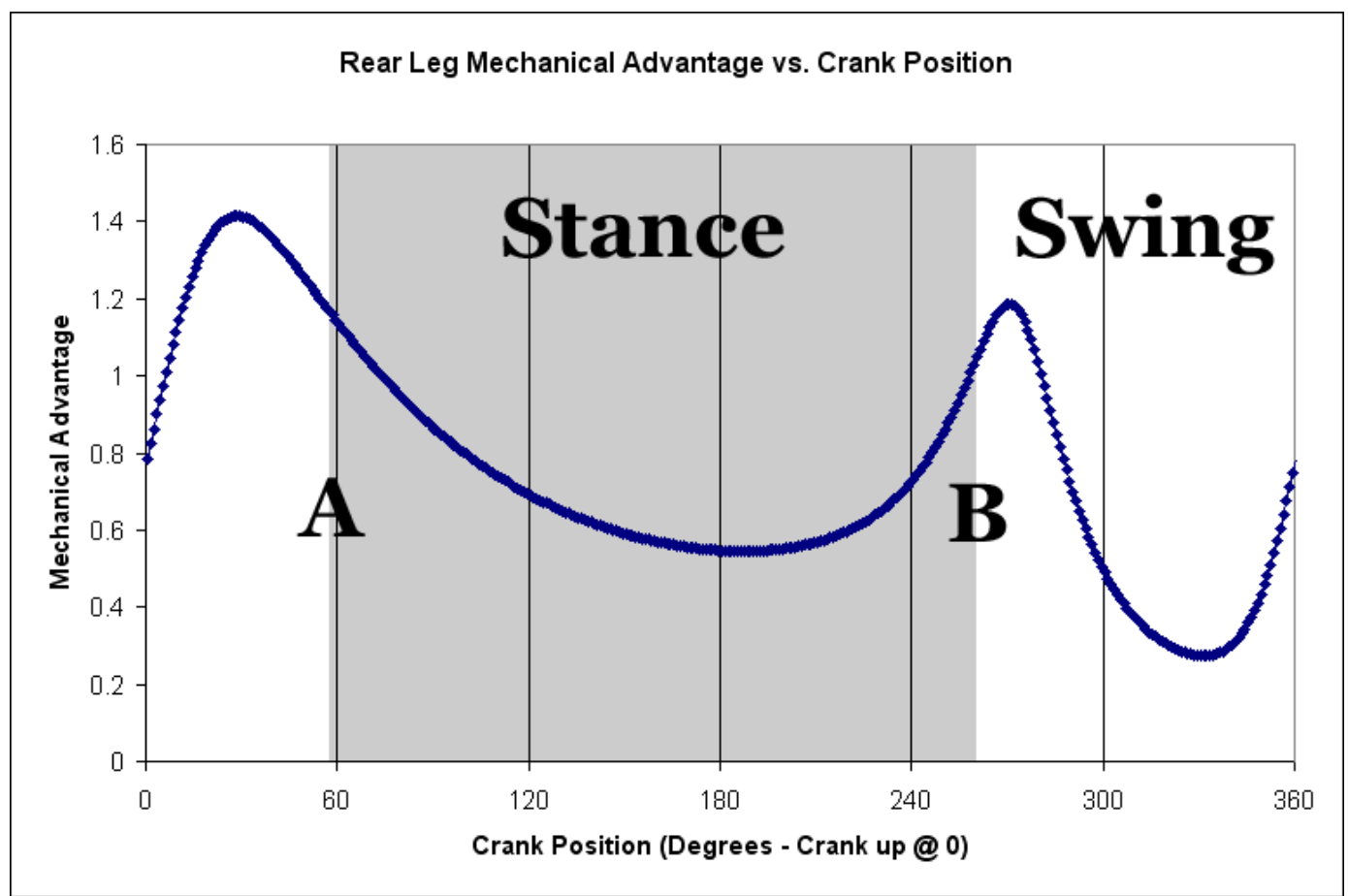

Figure 3-38. Rear Leg Mechanical Advantage vs. Crank Position

The mechanical advantage of the rear leg was plotted against crank position in Figure 3-38. The minimum mechanical advantage during stance is 0.545 , meaning that the output force at the foot is $54.5 \%$ or larger than the input force during that phase. This minimum value is almost $25 \%$ higher than the minimum mechanical advantage of the middle leg in stance, and over 80\% higher than the minimum seen in the front leg during stance.

The minimum mechanical advantage of the mechanism in swing is 0.274. The mechanical advantage reached lower values in swing than in stance due to the higher horizontal velocity of the foot in swing.

\subsubsection{Mechanism Construction}

The links of the mechanisms were machined out of aluminum. The tibia was machined from $0.635 \mathrm{~cm}$ thick aluminum plate. 
The crank and rocker links were each made out of two identical pieces that were machined from $0.476 \mathrm{~cm}$-thick aluminum plate. One part of the crank is mounted to the right side of the tibia, and the other identical part of the crank is mounted to the left side of the tibia (see Figure 3-39). By supporting the crank from both the right and left, bending loads created in the joints are reduced. The joints are weaker than the links themselves, and reduction of bending loads allows the joints to transmit more load without binding or becoming damaged. The rocker link of each leg was constructed in the same manner.

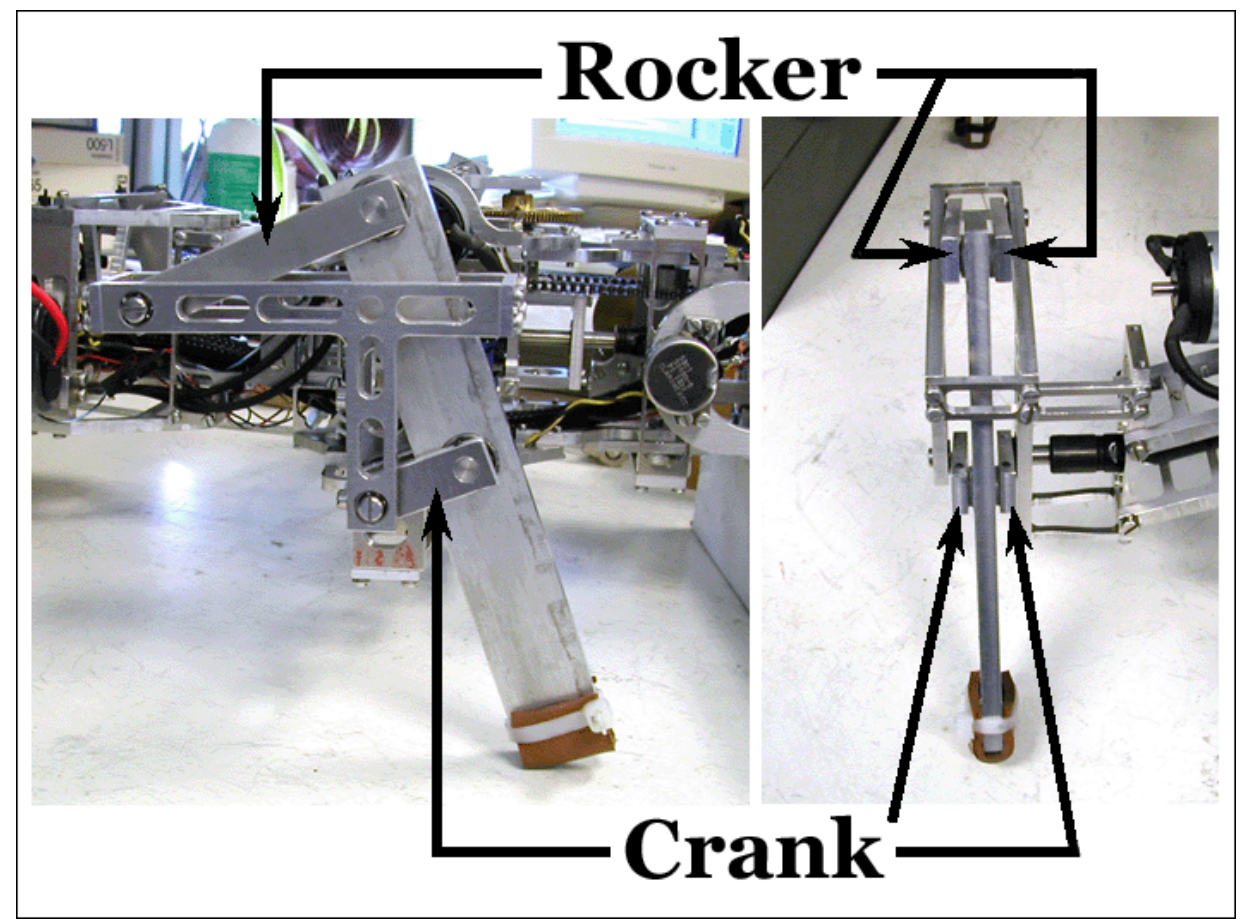

Figure 3-39. Crank and Rocker Each Consist of Two Identical Pieces

The joints themselves were constructed from precision ground $0.635 \mathrm{~cm}$-diameter steel shafts that are fixed to the crank or rocker links. These shafts pass through ball bearings that were press-fit into the tibias. This arrangement is easy to construct, inexpensive, and easy to disassemble for maintenance. It also restricts the joints to only one degree of freedom, and keeps the mechanism operating in a plane. 
The passive pin joints between the mechanism and the chassis of the robot are formed by $0.318 \mathrm{~cm}$-diameter shoulder screws passing through ball bearings that were press-fit into the chassis.

Delrin bushings placed between the links prevent the aluminum parts from coming into contact with each other as the mechanism moved. 


\section{Leg Compliance}

\subsection{The Need for Leg Compliance}

Cockroach legs have inherent passive stiffness and damping properties, which were shown in an experiment in which a large impulsive force too rapid to be counteracted by the nervous system was absorbed by the passive compliance of the legs [19]. These passive properties stabilize the cockroach and reduce the forces that are transmitted to the body, which are the exact reasons that passive leg compliance is desirable on the robot.

Additionally, previous robots have shown that a lack of passive stiffness and damping properties is undesirable. Shock and vibration loosens fasteners and causes mechanical failures.

Feet may also fail to gain proper traction without these properties. When the feet make contact with an obstacle, a stiff system may bounce off of the obstacle, instead of allowing the feet to hit the obstacle and remain in contact with it. This is precisely the behavior observed when MechaRoach I encounters an obstacle.

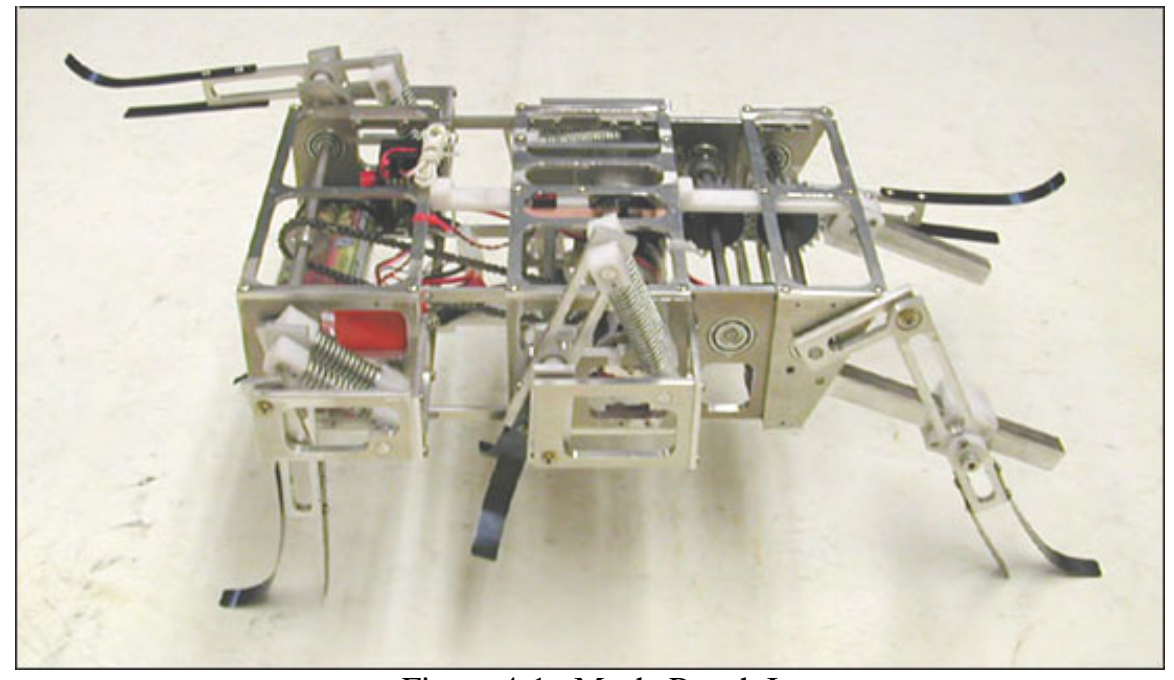

Figure 4-1. MechaRoach I

The tarsi of MechaRoach I (shown in Figure 4-1) were created from strips of spring steel. The resulting legs have a very high stiffness with almost no damping. When the 
legs of MechaRoach I encounter an obstacle, the feet often bounce off, preventing the feet from finding a foothold and often completely preventing the robot from surmounting the obstacle. The lack of damping in the legs often could be overcome by operating the robot on a carpeted surface, which provided damping of its own and reduced the stiffness of the interaction between the feet and the substrate. Lowering the passive stiffness and increasing the passive damping of the legs and feet could have allowed the robot to operate properly on a wider variety of surfaces.

The body of MechaRoach I also experiences large vertical displacements during walking. The robot walks in an alternating tripod gait, and as one tripod of support reaches the end of its stance phase, the robot drops down very quickly onto the other tripod of support. This drop onto the other tripod creates large forces that are delivered to the body of the robot, which causes frequent mechanical failures and the loosening of fasteners. A reduction in leg stiffness and an increase in passive damping would have acted like shock absorbers in a car, and could possibly have prevented a lot of the mechanical failures.

Whegs ${ }^{\mathrm{TM}}$ VP [41] could have also benefited from passive stiffness and damping properties. It is shown in Figure 4-2. 


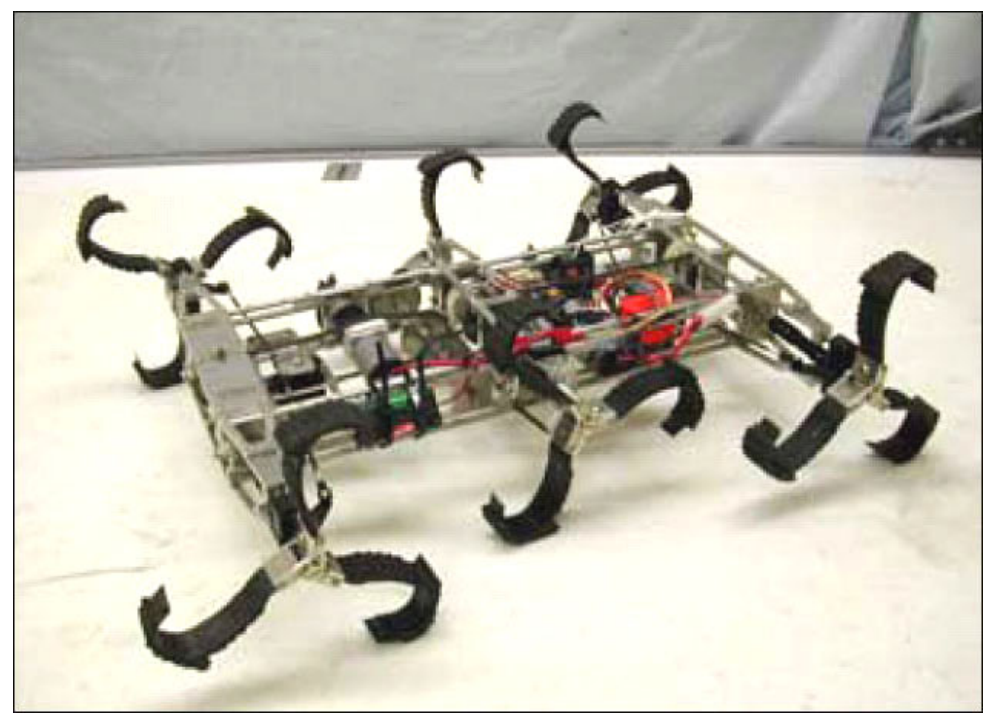

Figure 4-2. Whegs VP [41]

Like MechaRoach I, the feet on Whegs ${ }^{\mathrm{TM}}$ VP were made from strips of spring steel. The strips create a relatively high stiffness interaction between the feet and the ground, and provide virtually no damping. These strips are curved and coated in rubber, for the purpose of allowing the robot to gain traction on slippery surfaces.

Unfortunately, on high stiffness surfaces that are slippery, the lack of damping prevents the feet from gaining a proper foothold, even when the surface is flat and horizontal. For instance, when the robot is operated on linoleum, each foot hits the ground and then bounces back up into the air. The wheel-legs continue to rotate and then the next foot hits the ground and bounces back into the air, and the process repeats. The result is that all of the wheel-legs are rotating, but the chassis is unable to make forward progress. Like MechaRoach I, the addition of passive damping properties could have allowed the robot to operate on a wider variety of surfaces. 


\subsection{Leg Compliance on MechaRoach II}

Leg compliance on MechaRoach II is provided by shock absorbers placed in series between the chassis and its legs. The shock absorbers themselves are from a Team Associated Threaded Shock Kit for TC3 Touring Car. By using this product, all of the compliance properties are easily adjustable. The bump and rebound damping can be adjusted by filling the shock absorber with heavier or lighter oil. The oil used is Team Associated Silicone 50 Weight Shock Oil.

The stiffness can be adjusted by installing springs with a higher or lower spring rate. The springs that were used are Team Associated TC3 Touring Car Springs, which have a stiffness of $7005 \mathrm{~N} / \mathrm{m}$ (40 lbs/in).

A threaded collar allows the spring preload to be adjusted. Preload is necessary to prevent droop travel. Droop travel occurs when the weight of the chassis causes a steady state compression of the spring. If the left front leg is in stance, and the weight of the robot is compressing the spring of that leg, it will reduce the reaching height of the right front leg, which may prevent the robot from reaching over an obstacle.

Each shock absorber is very light $(14 \mathrm{~g})$, which is certainly lighter than what could have been manufactured with the equipment currently available in-house.

Figure 4-3 shows a diagramed view of the components that provide compliance to a leg. 


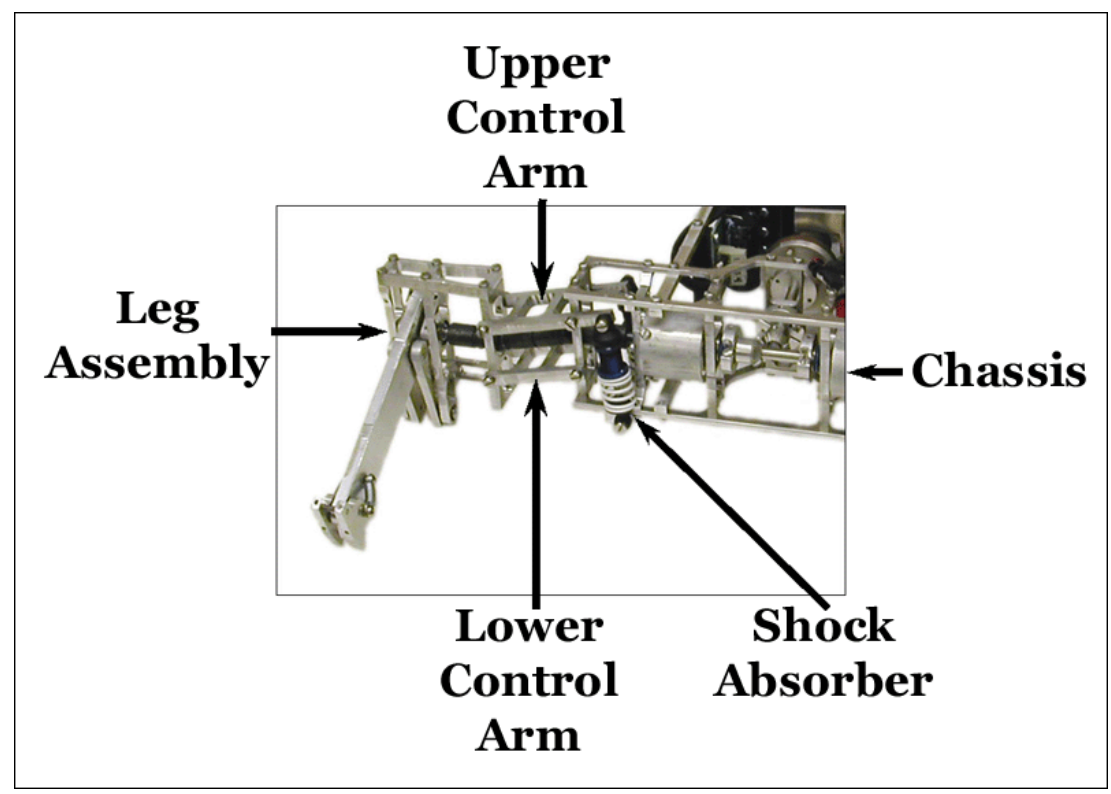

Figure 4-3. Front Leg Compliance Components

The leg assembly connects to the chassis through upper and lower control arms. The control arms are attached to the leg assembly and the chassis through passive pin joints. The upper control arm acts as a lever, with the leg assembly at one end, the shock absorber on the other end, and the pivot in between.
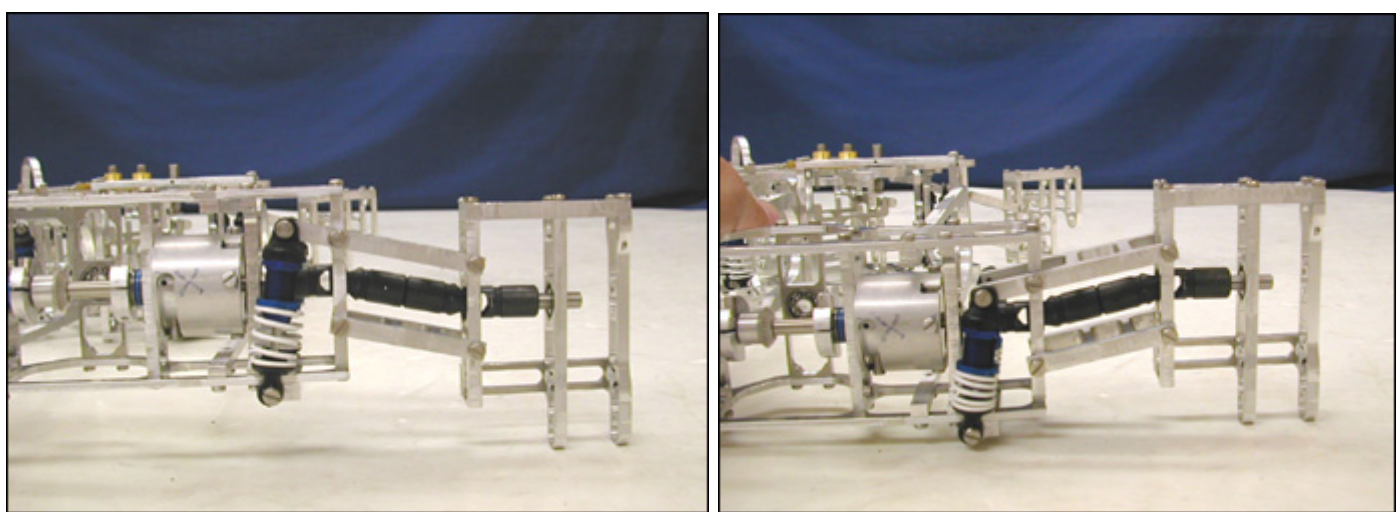

Figure 4-4. Leg Compliance in Unloaded (left) and Loaded (right) States

When the vertical load on the leg increases, the spring compresses, as seen in Figure 4-4. On the left part of the figure, there is no load being applied to the leg assembly. When a vertical load is applied to the leg assembly, as in the right part of the figure, the control arms are displaced, which compresses the spring. 
The pivot points of the upper and lower control arm are equally separated, and the control arms always remain parallel. The parallelogram configuration prevents camber changes as the suspension is compressed. The control arms were machined from $0.635 \mathrm{~cm}$ aluminum plate.

Double universal-joints allow the driveshafts to transfer torque to the leg mechanisms, regardless of suspension travel.

One of the positive features of the suspension design is that the compliance is not dependent upon leg orientation. The feet of the robot can make contact with an obstacle at any point in its trajectory, and compliance is available to ensure that the feet can gain traction. 


\section{Body Flexion}

One method that cockroachs employ to make the transition between horizontal and vertical surfaces is to flex their body downward between the first and second thoracic segments (see Figure 5-1). Body flexion keeps the cockroach's legs closer to the substrate, and prevents high-centering during transitions. When body flexion is prevented by a splint, the cockroach has to high-center and extend its front legs much further when making the transition from a vertical surface to a horizontal one [35].

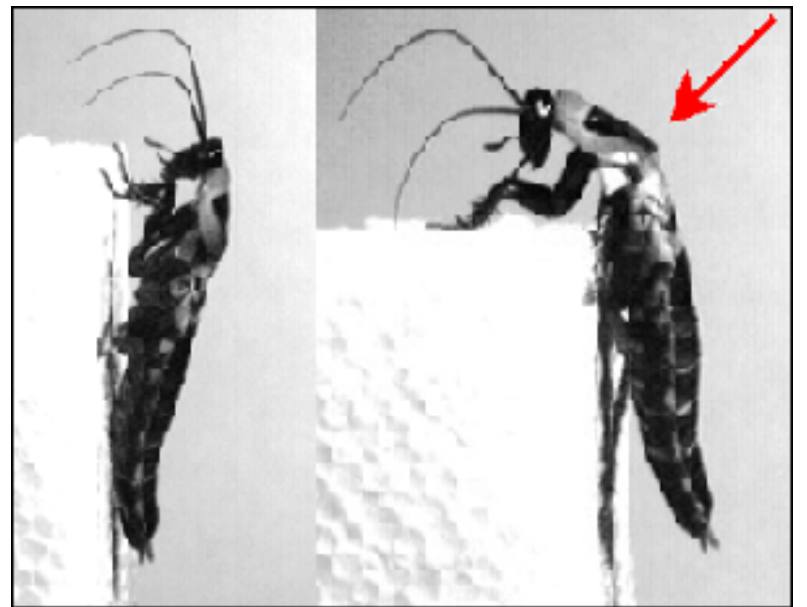

Figure 5-1. Cockroach Body Flexion Joint (Courtesy of Ritzmann Lab)

To perform a transition between a horizontal and a vertical surface, cockroaches do not bend their body flexion joint the other way, however. Instead, cockroaches rear their entire bodies upward using their middle legs [44]. 


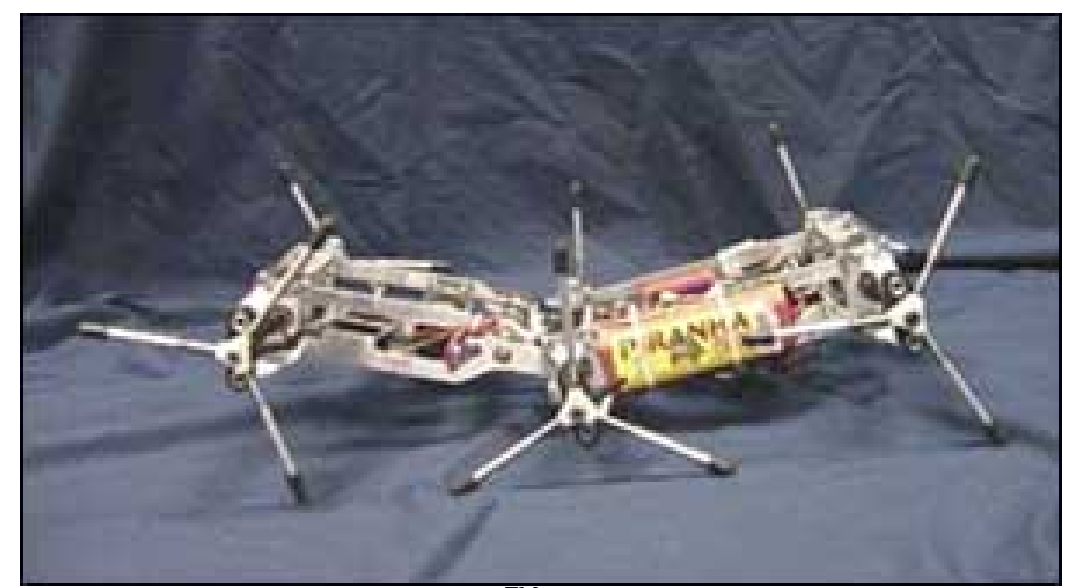

Figure 5-2. Whegs ${ }^{\mathrm{TM}}$ II Body Flexion Joint

Whegs ${ }^{\mathrm{TM}}$ robots have been developed with body flexion joints for the purpose of obstacle climbing. These robots have not been capable of extending their middle legs in order to rear their bodies upward. Instead, the body flexion joint is used to rotate the front of the robot both upward and downward. For example, the body flexion joint on Whegs ${ }^{\mathrm{TM}}$ II (see Figure 5-2) allowed the robot to climb obstacles that were 1.38 times as high as the spoke length [3].

MechaRoach II has a body flexion joint and uses it in the same way. In addition to increasing the robot's obstacle climbing ability, the usage of a body flexion joint was intended to help the robot make the transition between a horizontal and inclined surface.

The body flexion joint on MechaRoach II is powered by a 20W 32mm DC Maxon motor, and is shown in Figure 5-3. The motor is capable of $41.7 \mathrm{mNm}$ continuous torque output, and weighs $240 \mathrm{~g}$. The $6 \mathrm{~V}$ motor is actually supplied with $7.2 \mathrm{~V}$, and the torque is transferred through a Maxon GP32 gearhead that supplies a 3.7:1 gear reduction and weighs 118g. The motor is mounted longitudinally for weight distribution purposes. 


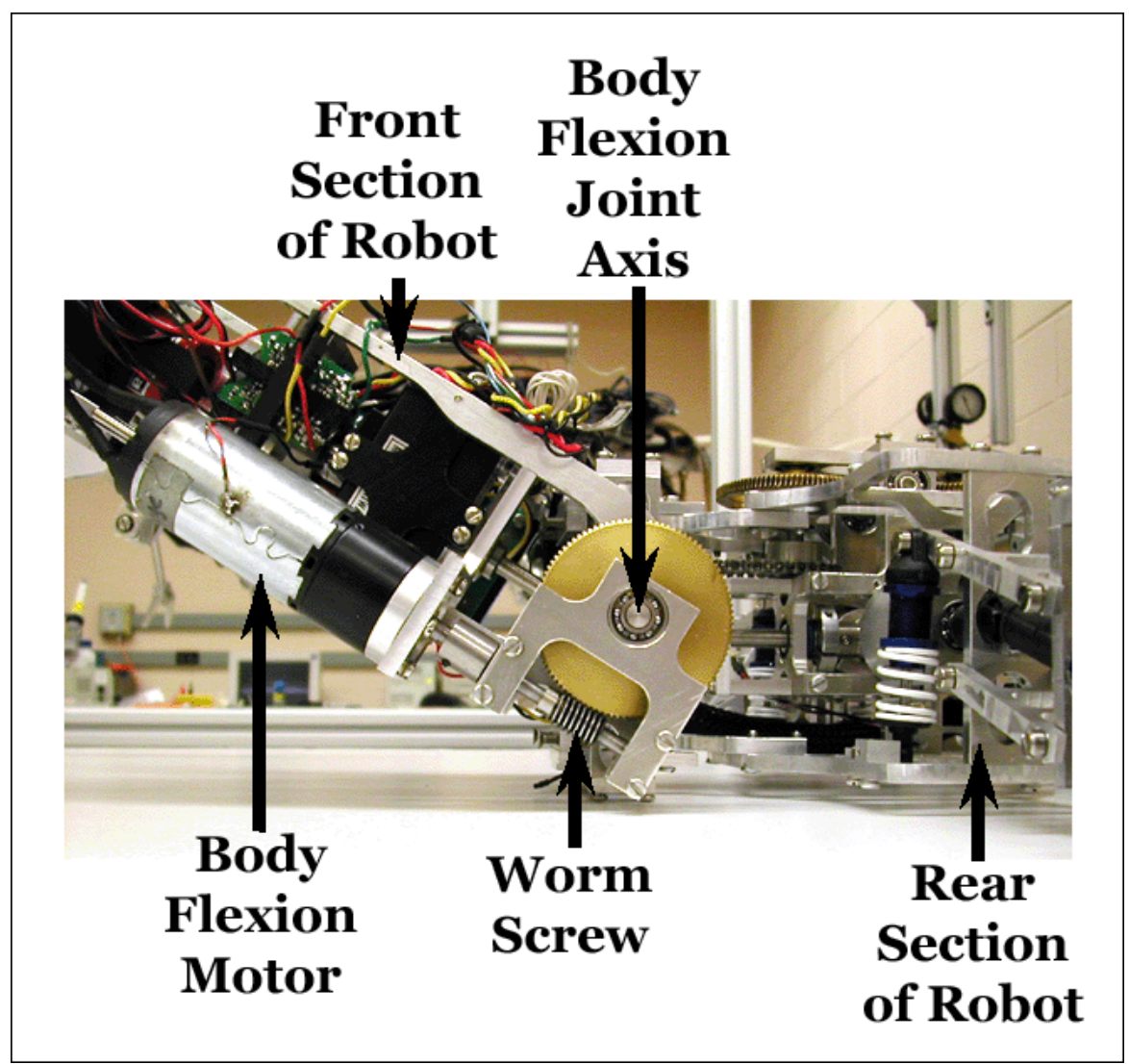

Figure 5-3. Side View of Robot and Body Flexion Joint

An additional 50:1 gear reduction is provided by a worm gear/worm screw combination. The worm gear is a 100 -tooth, 48 -pitch gear with a $5.29 \mathrm{~cm}$ pitch diameter. The worm screw has double thread starts and is 48 -pitch. A worm screw was used so that the steering system would be non-backdrivable. If the system could be back-driven, power would have to constantly be supplied to the motor in order for it to maintain its position. With the worm screw, once the system has reached the desired position, very little power is required to keep it there.

The motor is converted into a servo with the combination of a 5kOhm rotary potentiometer and a Lynxmotion SHB-01 speed controller. Initial operation at 7.2V indicated that the speed controller was overheating. Small aluminum fins were mounted to the speed controller to help dissipate heat. 
The nominal position of the body joint placed the front section of the robot aligned with the rear section in the sagittal plane (see bottom of Figure 5-4). The body flexion joint was capable of rotating the front section of the robot $+/-45$ degrees from the nominal position.
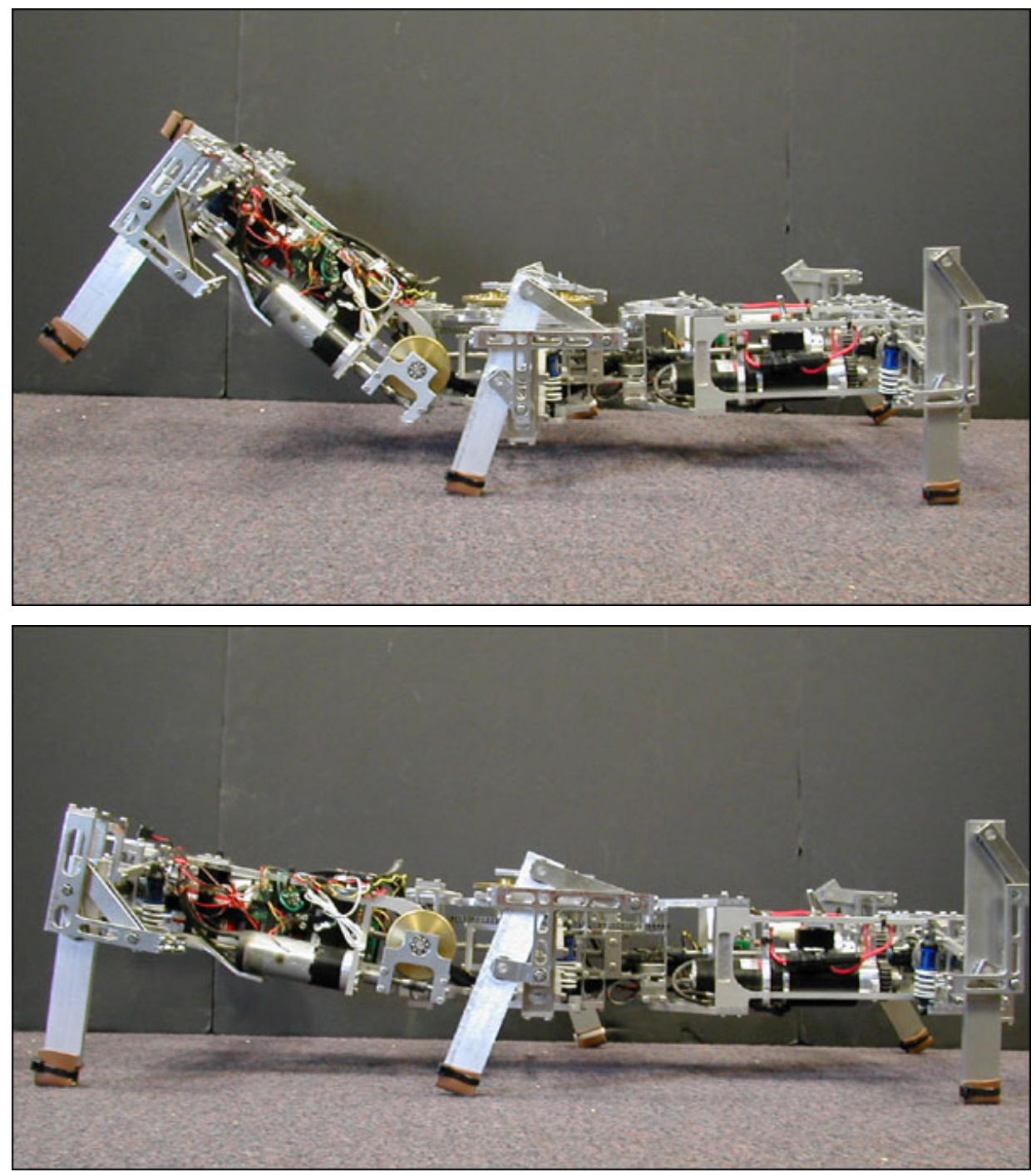

Figure 5-4. Body Joint Flexed and Unflexed

The front section of the robot weighs about 22N. The motor, through two gear reductions, should have been able to create a maximum of $7.7 \mathrm{Nm}$ of continuous torque, neglecting geartrain losses. As far as weight distribution, the worst-case scenario for the body joint would have been if all of the weight of the front section were at the opposite 
end of the body section than the body joint axis. This worst-case scenario would have placed the weight at $0.22 \mathrm{~m}$ away from the joint axis. The torque required to raise the front section of the robot would then be $4.8 \mathrm{Nm}$. Even in the worst-case scenario, there is enough power to lift the front of the robot. The center of gravity of the front section of the robot was actually located $0.16 \mathrm{~m}$ away from the body flexion joint axis, leaving plenty of torque to actuate the joint. 


\section{Gait Adapation}

Cockroaches normally walk in an alternating tripod gait, where the front and rear legs on one side of the animal move in phase with the middle leg on the other side [47]. Cockroaches bring their front, middle, and rear leg pairs into phase when climbing large obstacles, which increases their climbing ability and stability [44]. Increased climbing ability and stability are the exact reasons that gait adaptation is desirable on the robot.

The contralateral legs on MechaRoach II start out 180 degrees out of phase for the alternating tripod gait. The contralateral leg pairs are allowed to come into phase while climbing by the usage of passive torsionally compliant devices.

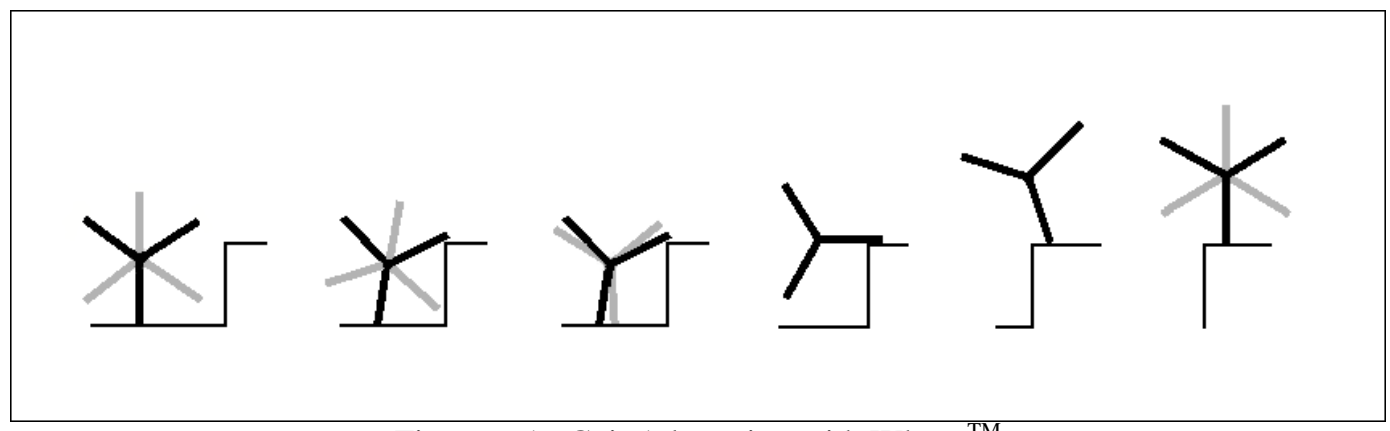

Figure 6-1. Gait Adaptation with Whegs ${ }^{\mathrm{TM}}$

It is easiest to see the effect of passive torsionally compliant devices on a Whegs ${ }^{\mathrm{TM}}$ robot. In Figure 6-1, the right leg is in black, while the left leg is in gray. In order to create an alternating tripod gait, the contralateral legs of a Whegs ${ }^{\mathrm{TM}}$ robot start 60 degrees out of phase, as they are on the left side of the figure. When one of the legs makes contact with an obstacle, the passive torsionally compliant device allows that leg to stop rotating, while the other leg continues to rotate. When both legs have made contact with the obstacle, they work together to lift the robot up and over the obstacle. Once the robot has reached the top of the obstacle, the legs return to being 60 degrees out of phase. 


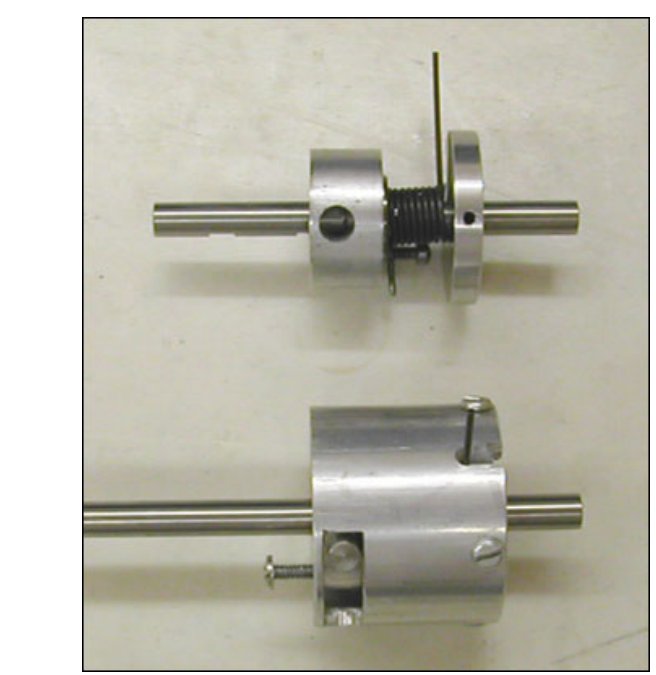

Figure 6-2. Passive Torsionally Compliant Device

Figure 6-2 shows the passive device that allows for gait adaptation in MechaRoach II. There are two views. A complete view of the device is shown at the bottom, and another view of the device without its outer housing is shown at the top. These devices are very simple and consist of only a handful of parts. The parts are labeled in an exploded view of the device in Figure 6-3.

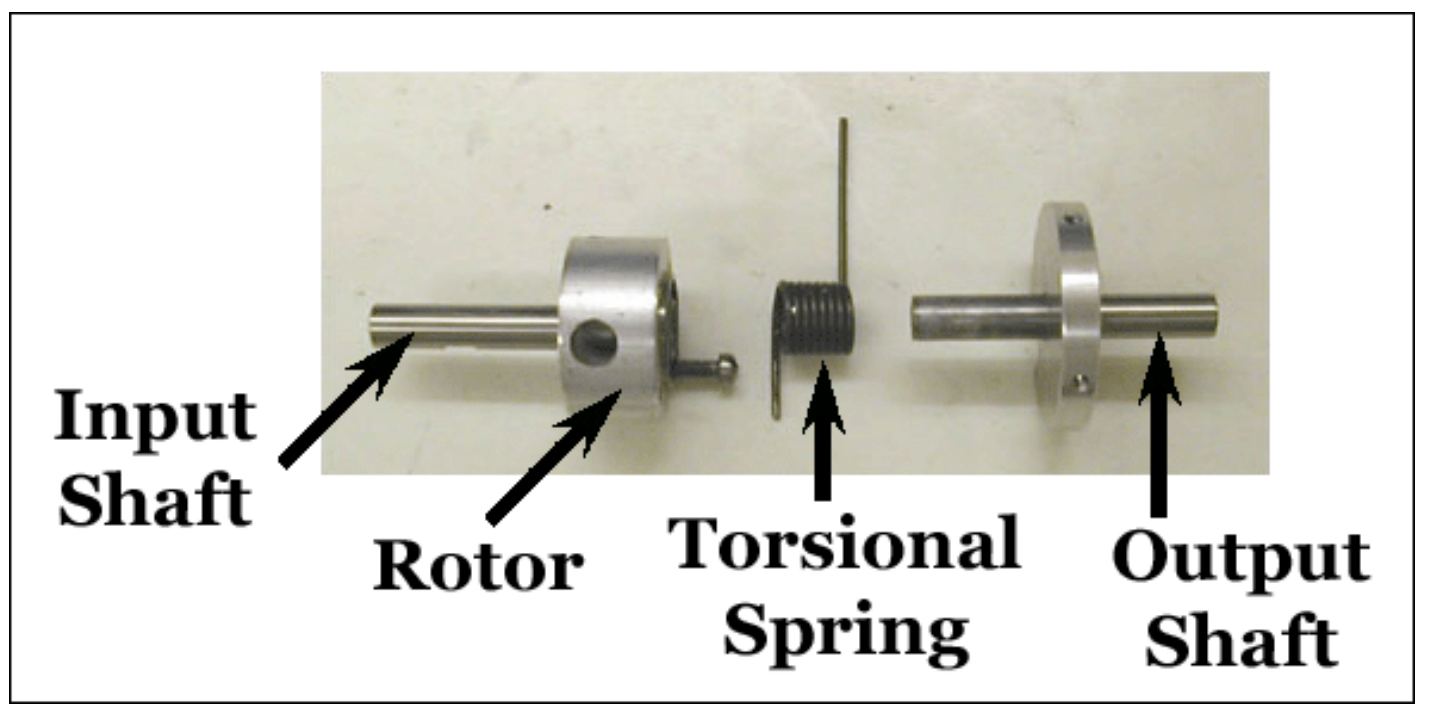

Figure 6-3. Exploded View of Torsionally Compliant Device

Each leg of the robot is connected to the drive motor through a passive torsionally compliant device. The device consists of five major components. Torque from the motor comes in from the input shaft, which was made from a precision ground steel shaft, 
$0.635 \mathrm{~cm}$ in diameter. The torque is then transferred from the input shaft to the rotor. The rotor was machined from $2.54 \mathrm{~cm}$-diameter, solid, round, aluminum stock. Each rotor also has a ball bearing that supports the end of the output shaft, and allows the two shafts to spin freely and independently of each other. The rotor transfers torque to a torsional spring, which provides the restoring force that keeps the legs in an alternating tripod gait. The springs are listed as having a stiffness of $11.9 \mathrm{~N} /$ degree. The left legs of the robot use right-hand wound springs, while the right legs of the robot use left-hand wound torsional springs. The torsional spring transfers the torque to the outer housing of the device. The outer housing was machined from $3.81 \mathrm{~cm}$-diameter cylindrical tubing, with a $0.159 \mathrm{~cm}$ wall thickness. Finally, there is a pin that has been press-fit into each rotor. The pin rides in a slot in the outer housing, the limits of which limit the relative rotation allowed between the input and output shafts.

The devices are very simple to machine and assemble. Each device's total weight is 89g, with much of the weight in the steel input and output shafts. The inclusion of the bearing in the rotor allows the device to operate smoothly.

The contralateral legs on MechaRoach II are not 60 degrees out of phase like they would be on a Whegs ${ }^{\mathrm{TM}}$ robot. Instead, they are 180 degrees out of phase. Therefore, each torsionally compliant device has to allow the output shaft to rotate up to 180 degrees relative to the input shaft.

The torsionally compliant devices were among the first of the components designed and machined for MechaRoach II. Before the rest of the robot was completed, the front legs of MechaRoach I were modified to use these devices to make sure they would work as intended with 4-bar mechanism legs. MechaRoach I originally did not employ 
methods for gait adaptation. Only the front legs of MechaRoach I were modified to use these devices, because extensive redesign of the chassis would have been required in order to install them on the middle and rear legs.

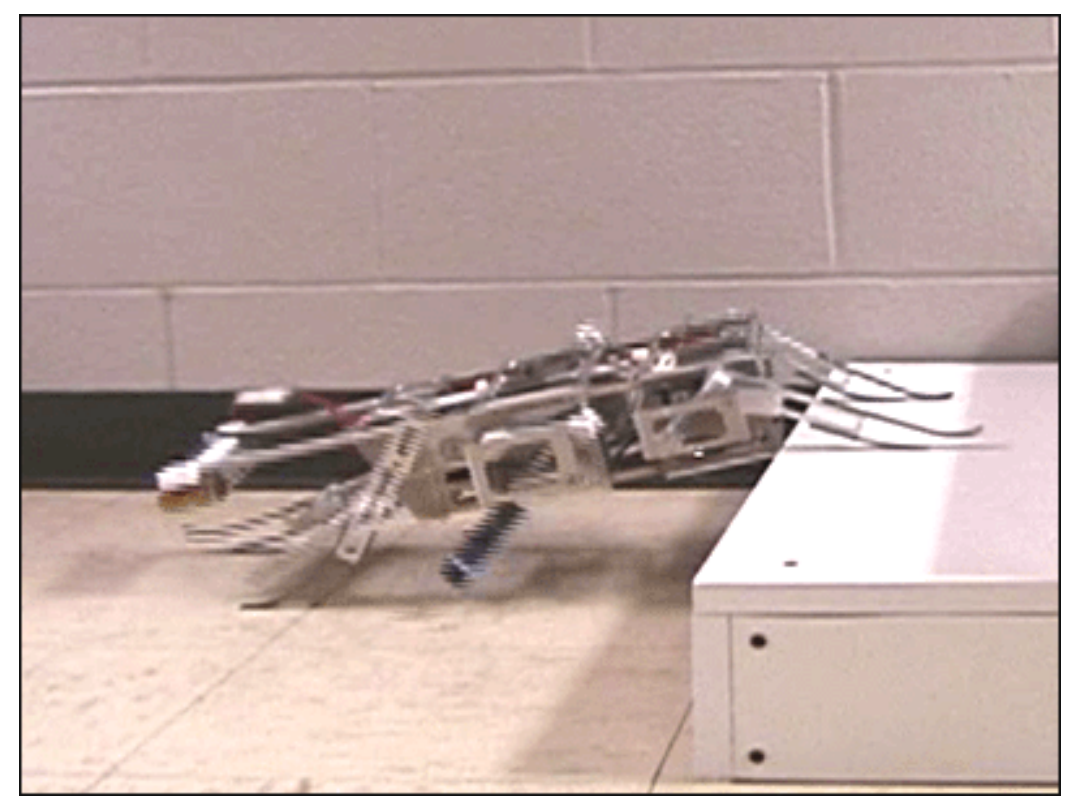

Insert Figure 6-4: MechaRoach I Gait Adaptation

Video was taken of MechaRoach I climbing on an obstacle, and the video was viewed at a reduced speed to verify the behavior was as expected. The video showed the front legs starting 180 degrees out of phase. The legs were then brought into phase to work together to lift the robot onto the top of an obstacle (see Figure 6-4). The devices then operated as expected and returned the legs to an alternating tripod gait afterward. Due to the success of the devices on MechaRoach I, the development of MechaRoach II continued. 


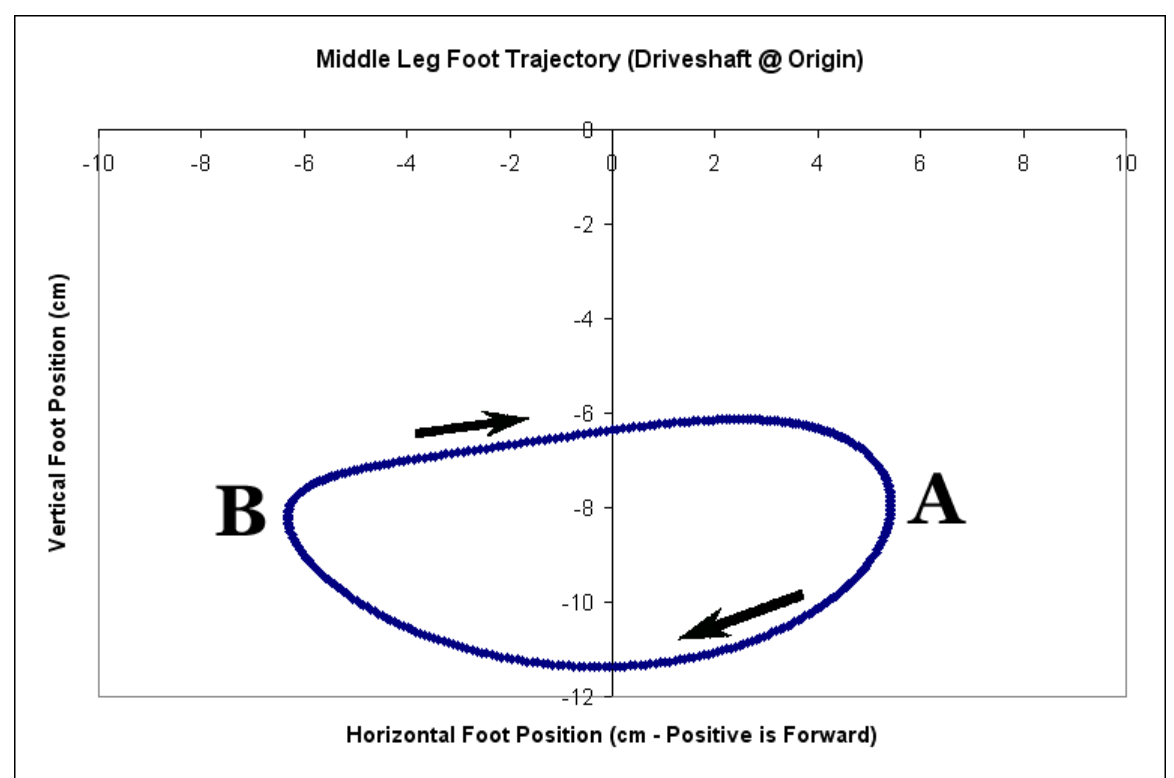

Figure 6-5. Middle Leg Footpath Trajectory

The torsionally compliant mechanisms have another major effect on locomotion that is not directly involved in gait adaptation. Figure 6-5 shows the footpath of the middle leg. At point A, heel strike occurs and the leg is pushing the foot downward. In order to continue with the rest of the stance phase, the foot must move downward before moving rearward. However, since the leg already is in contact with the ground, it must lift the body of the robot upward before continuing with its trajectory. The weight of the robot therefore opposes the direction the leg is trying to move the foot in. The result is that the spring in the torsionally compliant device is wound up at heel strike. The spring winds up until enough torque is transferred through it to allow the body of the robot to be lifted up and for the leg to continue the rest of its stance trajectory.

When the trajectory of the foot reaches point $\mathrm{B}$ in the figure, the foot lifts up off the ground, and the weight of the robot is transferred to other legs. Because there is no longer a force resisting the leg's movement, the spring in the torsionally complaint device rapidly unwinds. 
As a result, each leg is able to spend a higher percentage of each cycle in stance than they would have been able to without the torsionally compliant devices. Each leg also spends less time in swing, when they are not able to support the robot.

The analysis of the foot trajectories in Section 3.7 had assumed a constant crank velocity. If the drive motor was driven at a constant speed and torsional compliance had not been installed on the robot, the crank of each leg mechanism would turn at a constant velocity. However, the torsionally compliant devices cause the cranks to have a varying speed, even with the drive motor running at a constant speed. During each cycle, the cranks are brought to a stop at heel strike. The cranks also rapidly accelerate after toe off as the springs are released. 


\section{Vacuum Adhesion}

\subsection{Vortex Regenerated Air Movement}

In order for the robot to climb a sharply inclined or vertical surface, the robot would have to create an attachment or adhesive force to keep it in contact with the substrate. One method proposed to create this attachment force was to create a low-pressure area underneath the robot. The legs then would only have to provide propulsive forces.

The VRAM (Vortex Regenerated Air Movement) Mobile Robot Platform [17] uses an impeller spun at high angular velocity under a fixed housing. The vanes on the impeller cause the air underneath to spin, and the angular velocity causes the air to move away from the axis of rotation, creating a low-pressure area. Only the higher-pressure ambient air creates a centripetal force.

Balancing this centripetal force with the momentum moving the air away from the axis of rotation allows a theoretical calculation for the adhesion force that would be generated by the impeller (see Figure 7-1). In the figure, $r$ is the radial distance away from the axis of the impeller that an infinitesimal volume is located, $\mathrm{v}$ is that volume's instantaneous linear velocity, and $\mathrm{P}$ is the air pressure at the location. 


$$
\frac{\partial P}{\partial r}=\frac{\rho v^{2}}{r}=\rho \Omega^{2} r
$$

Integrating the above,

$$
P=\rho \Omega^{2} r^{2}+C
$$

Pressure at boundary $\quad P(r=R)=P_{A T M}$

$$
\text { Therefore } P_{A T M}=\rho \Omega^{2} R^{2}+C
$$

Subtracting (1) from (2),

$$
\begin{gathered}
P-P_{A T M}=-\left[\rho \Omega^{2}\left(R^{2}-r^{2}\right)\right] \\
F=\int\left(P_{A T M}-P\right) d A=\int\left(P_{A T M}-P\right) 2 \pi r d r
\end{gathered}
$$

Inserting (3),

$$
F=\int 2 \pi \rho \Omega^{2}\left(R^{2}-r^{2}\right) r d r
$$

Integrating from 0 to $\mathrm{R}$,

$$
F=2 \pi \rho \Omega^{2}\left[\frac{R^{2} r^{2}}{2}-\frac{r^{4}}{4}\right]_{0}^{R}=\frac{\pi \rho \Omega^{2} R^{4}}{2}
$$

Figure 7-1. Theoretical Calculation of Adhesion Force [32]

So, the adhesion force is given by

$$
\mathrm{F}=\pi \cdot \rho \cdot \Omega^{2} \cdot \mathrm{R}^{4} / 2
$$

where

$$
\begin{aligned}
& \rho=\text { air density } \\
& \Omega=\text { angular velocity } \\
& \mathrm{R}=\text { impeller radius }
\end{aligned}
$$

Multiple impellers could have been used on the robot to generate the necessary adhesion forces. For instance, one or more impellers could have been located on the 
front section of the robot, while one or more could have also been located on the rear section.

\subsection{Tested Impellers}

13 impellers were machined out of LDPE (Low Density Polyethylene) to evaluate the possibility of using this technology on MechaRoach II. Because of the complex shapes of the vanes on the impellers, impellers were CNC (Computer Numerical Control) machined.

The impellers were machined before any of the work on MechaRoach II was performed, and the Hurco VM1 that was used to machine the robot was not available yet. At the time that the impellers were machined, a Roland PNC-3100 CNC milling machine was the largest CNC available in-house, and a $10.16 \mathrm{~cm}$ diameter impeller was the largest that could be machined with it. Usage of a larger impeller would have been beneficial, as it has already been predicted in Figure 7-1 that the suction force that can be generated increases with (radius) ${ }^{4}$.

The impellers consist of vanes and a $0.076 \mathrm{~cm}$ thick backplane. Some impellers also have housing attached to them and housing-like rings (see Figure 7-2). Each impeller and all of its parts were entirely machined out of a single piece of LDPE. 


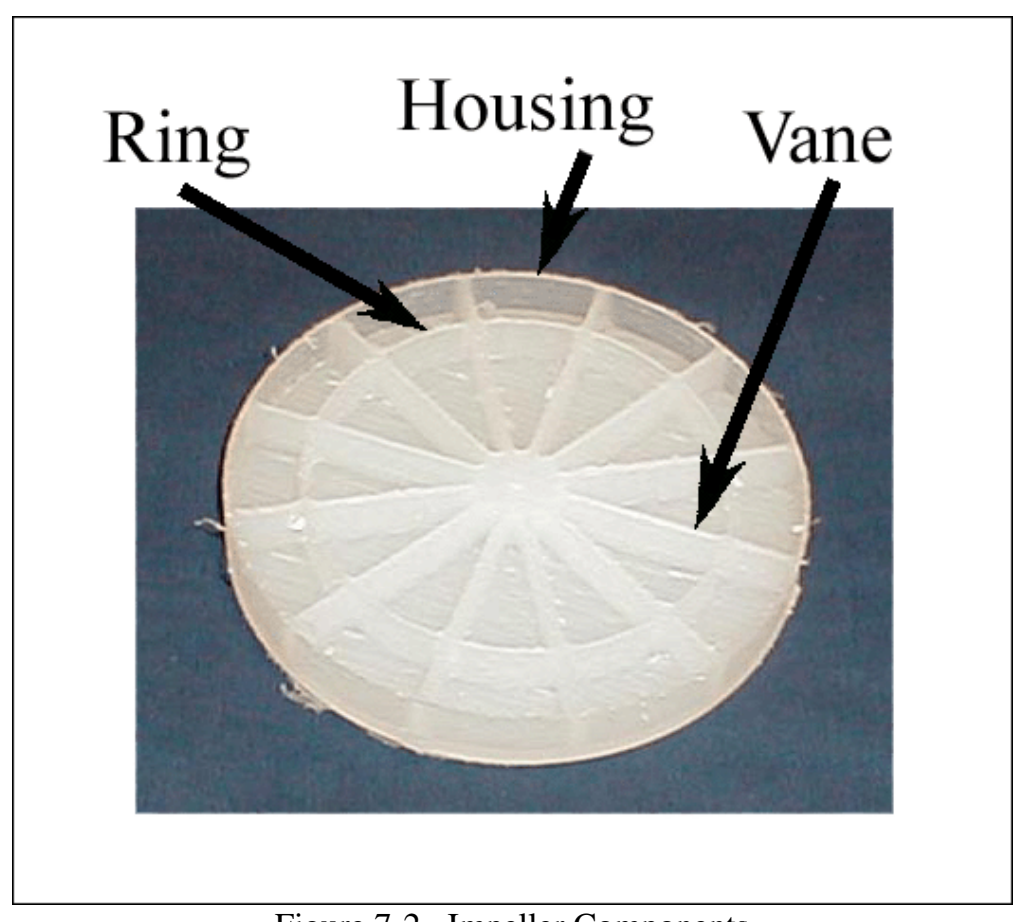

Figure 7-2. Impeller Components

The 13 impellers that were created included 1 impeller that was spun under a fixed housing, 10 in which the housing was integrated into the impeller, and 2 that had no housing whatsoever. The impellers were created in order to determine which features on the impeller were the most important ones. The primary difference between the various impellers, other than housing, is the number, shape, and length of their vanes. The presence of housing and the shape and number of vanes could affect how efficiently the impellers move the air underneath them, which is why so many rotors were created for testing. 

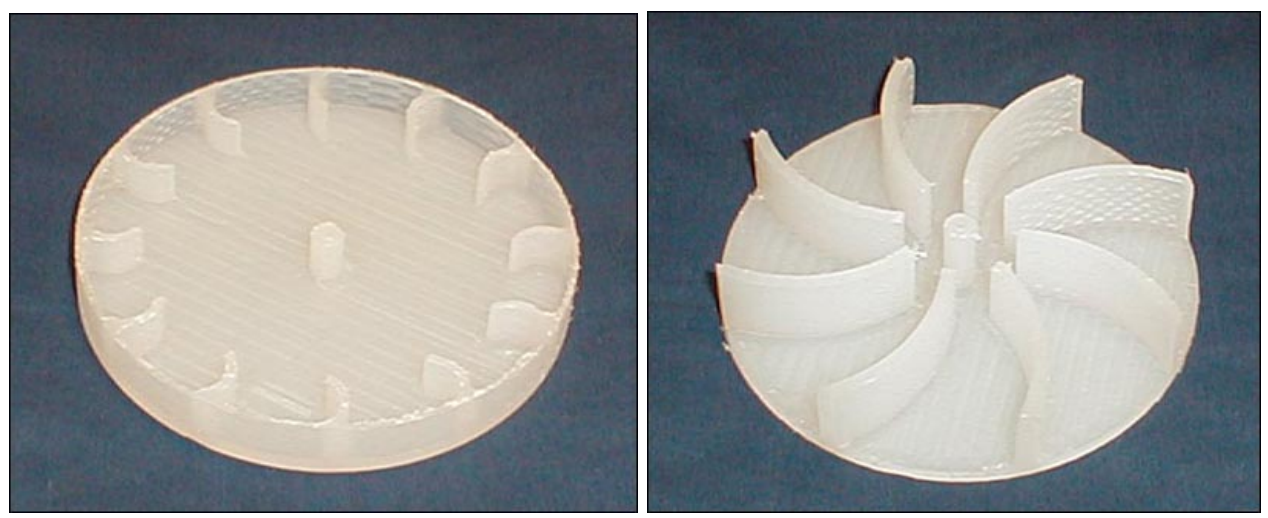

Figure 7-3. Impeller \#1 (left) and Impeller \#2 (right)

Impeller \#1 is shown in Figure 7-3. Its total thickness measures $1.27 \mathrm{~cm}$, has a diameter of $10.16 \mathrm{~cm}$, twelve short curved vanes, and the housing is integrated into the impeller.

Impeller \#2 has a thickness of $1.9 \mathrm{~cm}$, a diameter of $10.16 \mathrm{~cm}$, eight curved vanes that reach almost to the axis of rotation, and no housing.
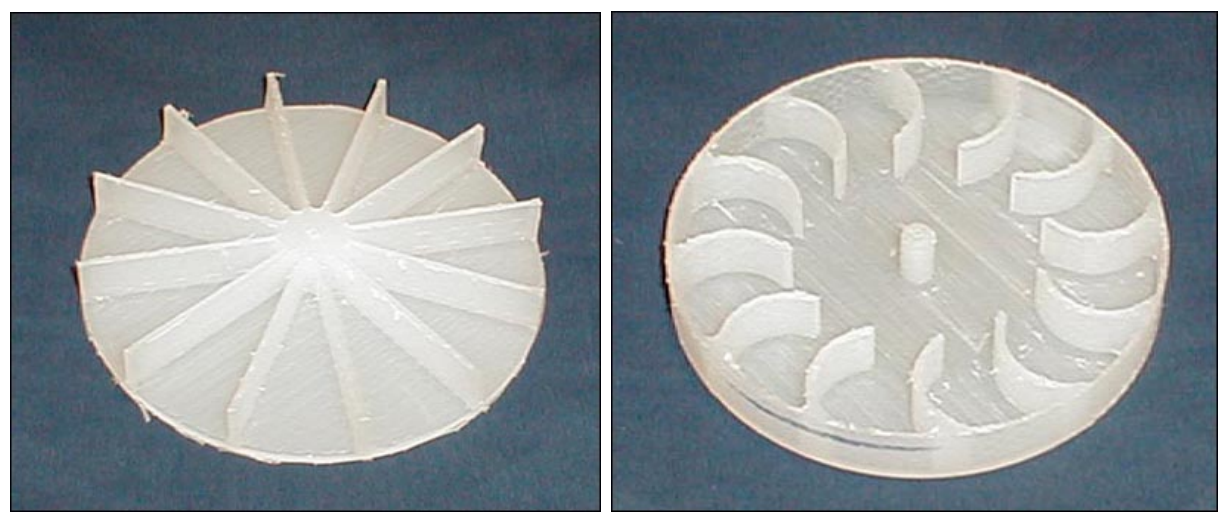

Figure 7-4. Impeller \#3 (left) and Impeller \#4 (right).

Both impellers \#3 and \#4 have a thickness of $1.27 \mathrm{~cm}$, and a diameter of $10.16 \mathrm{~cm}$. Impeller \#3 has twelve long, straight blades that reach all of the way to the axis of rotation, and no housing. Impeller \#4 has 12 shorter, curved vanes and a housing that is fixed to the impeller. 

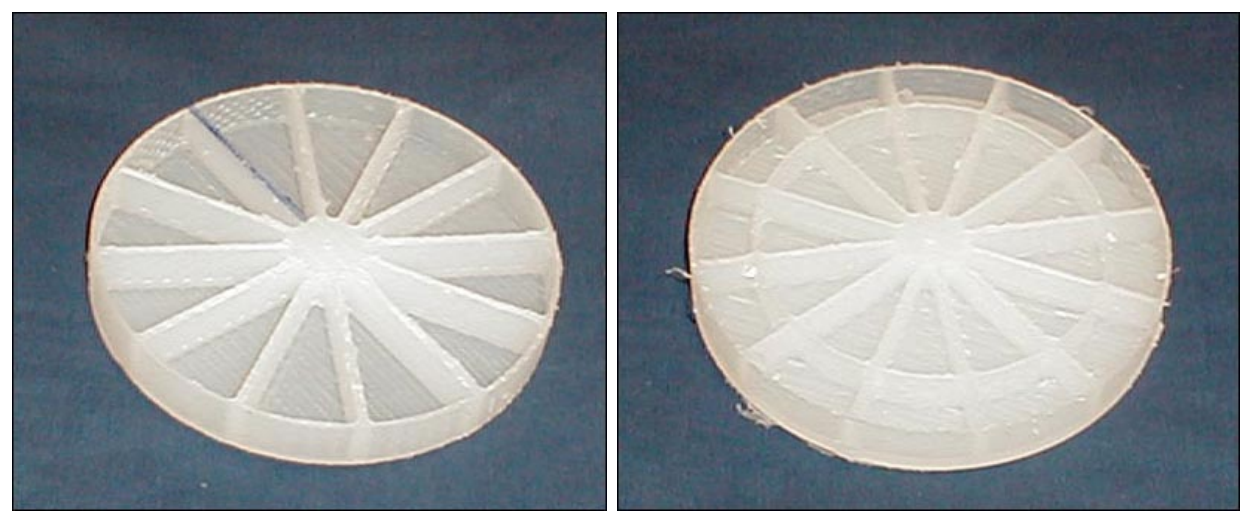

Figure 7-5. Impeller \#5 (left) and Impeller \#6 (right).

Impellers \#5 and \#6 both have a thickness of $1.27 \mathrm{~cm}$ and a diameter of $10.16 \mathrm{~cm}$, along with 12 long, straight blades and housings fixed to the impellers. Impeller \#6 also has another housing-like ring $1.9 \mathrm{~cm}$ inward of the actual housing.
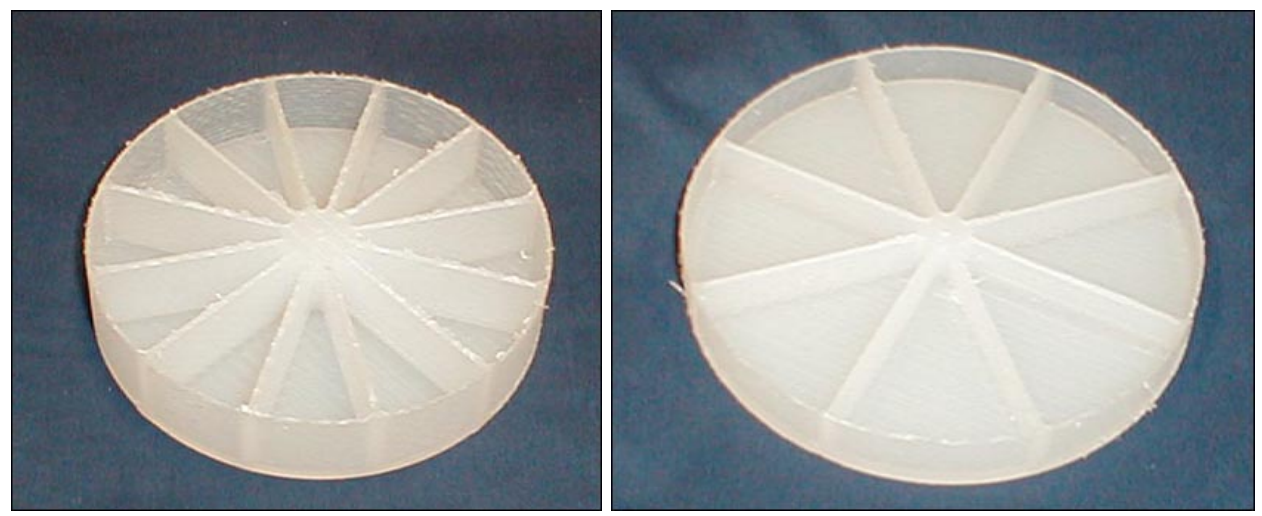

Figure 7-6. Impeller \#7 (left) and Impeller \#8 (right).

Impeller \#7 is $1.9 \mathrm{~cm}$ thick, $10.16 \mathrm{~cm}$ in diameter, and has 12 long, straight blades. It is very similar in design to impeller \#5, but thicker. Impeller $\# 8$ is $1.27 \mathrm{~cm}$ thick, 10.16cm in diameter, and has eight long, straight vanes. Both \#7 and \#8 have housings fixed on the impellers. 

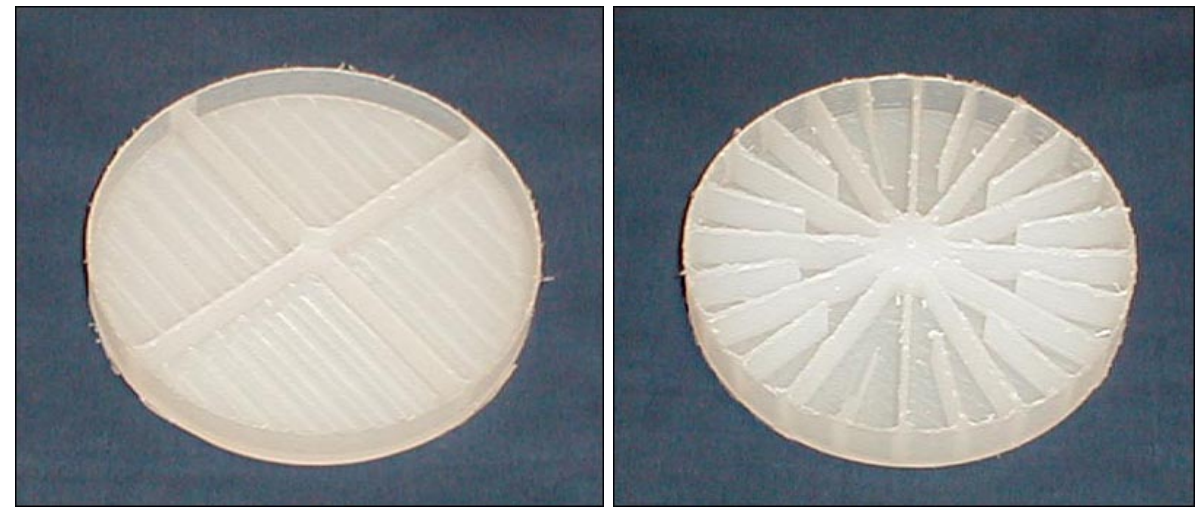

Figure 7-7. Impeller \#9 (left) and Impeller \#10 (right)

Impellers \#9 and \#10 are $1.27 \mathrm{~cm}$ thick, $10.16 \mathrm{~cm}$ in diameter, and have housings attached to them. Impeller \#9 features four long, straight vanes, while impeller \#10 has twelve long, straight blades, interspersed with twelve $1.9 \mathrm{~cm}$ straight blades located at the outer edge.

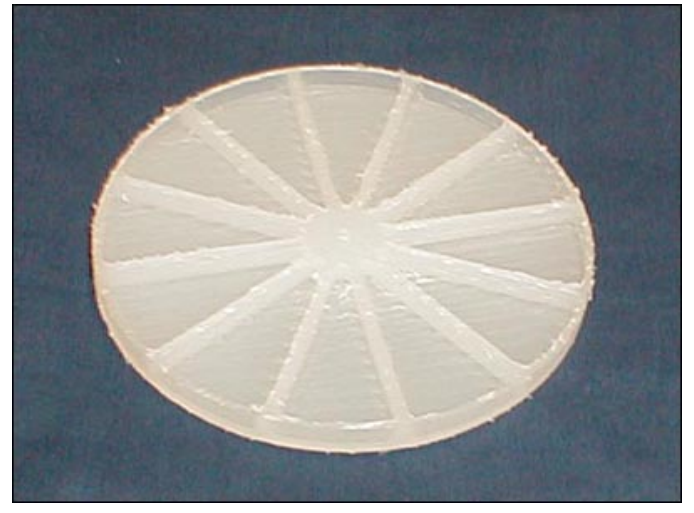

Figure 7-8. Impeller \#11.

Impeller \#11 is $0.64 \mathrm{~cm}$ thick, $10.16 \mathrm{~cm}$ in diameter, and has twelve long, straight vanes. The impeller's housing is integrated into its design. 


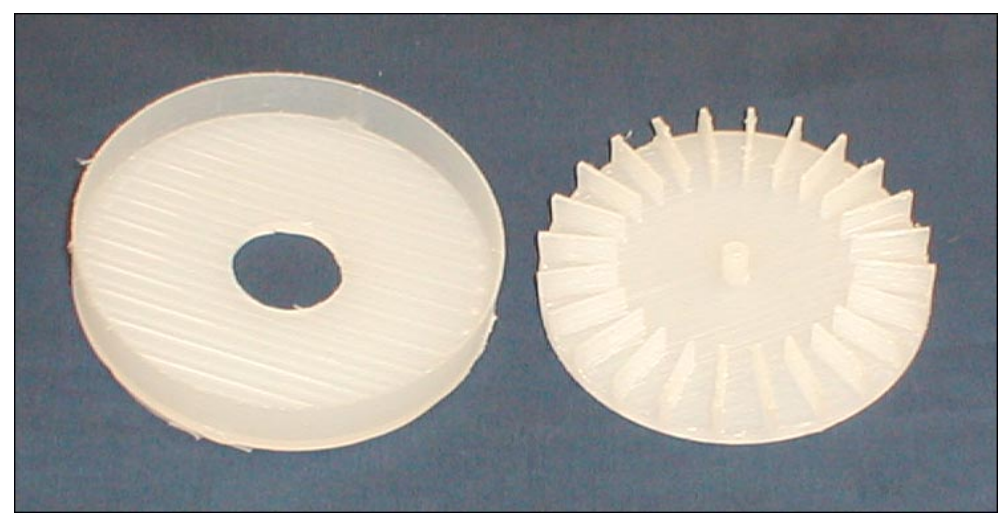

Figure 7-9. Impeller \#12.

Impeller \#12 is the only impeller tested that has a fixed housing. The housing is kept stationary while the impeller is spun underneath it. The impeller is $1.27 \mathrm{~cm}$ thick, $9.65 \mathrm{~cm}$ in diameter, and has twenty-four $1.9 \mathrm{~cm}$ straight vanes located at its outer edge. The impeller is slightly smaller in diameter than the previous 11 , because it has to fit and spin freely underneath the housing. The housing is $10.16 \mathrm{~cm}$ in diameter, which, again, was the largest that could be CNC machined in-house.

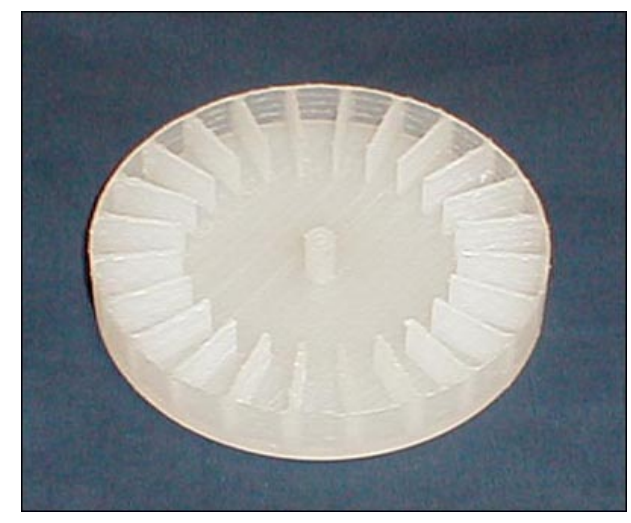

Figure 7-10. Impeller \#13.

Impeller \#13 is $1.27 \mathrm{~cm}$ thick and $9.65 \mathrm{~cm}$ in diameter. It also has twenty-four $1.9 \mathrm{~cm}$ straight vanes located at its outer edge, and a housing attached to the impeller.

The physical descriptions of the impellers are included in Table 7-1. 


\begin{tabular}{|l|l|l|l|l|l|l|l|l|}
\hline Impeller & Thickness & Diameter & \# of Vanes & Vane Length & Vane Type & Housing & Rings & Weight \\
\hline $\mathbf{1}$ & $1.27 \mathrm{~cm}$ & $10.16 \mathrm{~cm}$ & 12 & $1.27 \mathrm{~cm}$ & curved & on impeller & none & $29 \mathrm{~g}$ \\
\hline $\mathbf{2}$ & $1.9 \mathrm{~cm}$ & $10.16 \mathrm{~cm}$ & 8 & $4.45 \mathrm{~cm}$ & curved & none & none & $39 \mathrm{~g}$ \\
\hline $\mathbf{3}$ & $1.27 \mathrm{~cm}$ & $10.16 \mathrm{~cm}$ & 12 & $5.08 \mathrm{~cm}$ & straight & none & none & $25 \mathrm{~g}$ \\
\hline $\mathbf{4}$ & $1.27 \mathrm{~cm}$ & $10.16 \mathrm{~cm}$ & 12 & $1.9 \mathrm{~cm}$ & curved & on impeller & none & $22 \mathrm{~g}$ \\
\hline $\mathbf{5}$ & $1.27 \mathrm{~cm}$ & $10.16 \mathrm{~cm}$ & 12 & $5.08 \mathrm{~cm}$ & straight & on impeller & none & $32 \mathrm{~g}$ \\
\hline $\mathbf{6}$ & $1.27 \mathrm{~cm}$ & $10.16 \mathrm{~cm}$ & 12 & $5.08 \mathrm{~cm}$ & straight & on impeller & 1 & $37 \mathrm{~g}$ \\
\hline $\mathbf{7}$ & $1.9 \mathrm{~cm}$ & $10.16 \mathrm{~cm}$ & 12 & $5.08 \mathrm{~cm}$ & straight & on impeller & none & $38 \mathrm{~g}$ \\
\hline $\mathbf{8}$ & $1.27 \mathrm{~cm}$ & $10.16 \mathrm{~cm}$ & 8 & $5.08 \mathrm{~cm}$ & straight & on impeller & none & $33 \mathrm{~g}$ \\
\hline $\mathbf{9}$ & $1.27 \mathrm{~cm}$ & $10.16 \mathrm{~cm}$ & 4 & $5.08 \mathrm{~cm}$ & straight & on impeller & none & $31 \mathrm{~g}$ \\
\hline $\mathbf{1 0}$ & $1.27 \mathrm{~cm}$ & $10.16 \mathrm{~cm}$ & 24 & $5.08 \mathrm{~cm} / 1.9 \mathrm{~cm}$ & straight & on impeller & none & $33 \mathrm{~g}$ \\
\hline $\mathbf{1 1}$ & $0.64 \mathrm{~cm}$ & $10.16 \mathrm{~cm}$ & 12 & $5.08 \mathrm{~cm}$ & straight & on impeller & none & $23 \mathrm{~g}$ \\
\hline & & & & & & & & $29 \mathrm{~g}$ (rotor) \\
\hline $\mathbf{1 2}$ & $1.27 \mathrm{~cm}$ & $9.65 \mathrm{~cm}$ & 24 & $1.9 \mathrm{~cm}$ & straight & fixed & none & $28 \mathrm{~g}$ (housing) \\
\hline $\mathbf{1 3}$ & $1.27 \mathrm{~cm}$ & $9.65 \mathrm{~cm}$ & 24 & $1.9 \mathrm{~cm}$ & straight & on impeller & none & $32 \mathrm{~g}$ \\
\hline
\end{tabular}

Table 7-1. Physical Descriptions of the Thirteen Tested Impellers

\subsection{Lightweight Impeller Test Rig}

Initially, in order to quickly determine whether the impellers would be able to generate sufficient adhesion forces to a substrate, they were tested qualitatively using a lightweight test rig.

The rig consists of a DC hobby motor that is mounted in a Delrin frame. The Delrin frame has three legs protruding from it. The legs were made from music wire, and their purpose is to hold the hobby motor and frame at a fixed distance away from the substrate. The weight of the rig is $65 \mathrm{~g}$, including the DC hobby motor but not including the impeller. 


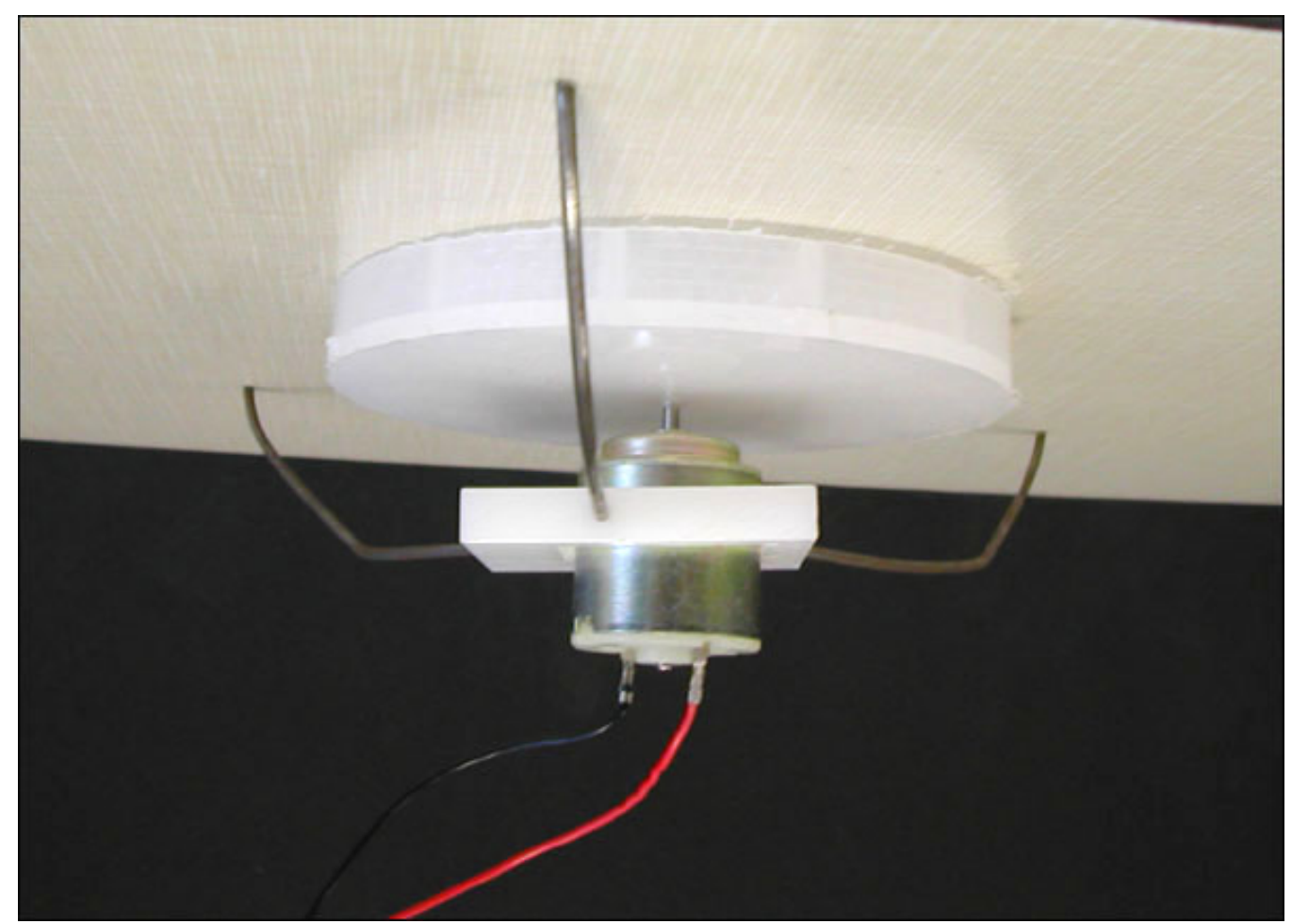

Figure 7-11. Lightweight Impeller Test Rig

The impeller was mounted on the output shaft of the DC hobby motor. The legs were then adjusted to hold the impeller at various distances away from the substrate. The motor was supplied with 3V from a Tektronix PS280 DC Power Supply. The test rig was placed upside down on the underside of a table as shown in Figure 7-11, such that the adhesion force being generated would try to support the entire weight of the test rig.

The spacing between the impellers and the substrate was adjusted, and the impellers were observed to generate maximum forces when the spacing was minimized. Only a few impellers (impellers \# 1,3,4, and 5) were able to generate enough force to hold the rig up against gravity. 


\subsection{Instrumented Impeller Test Rig}

\subsubsection{Test Rig Construction}

Although the qualitative tests with the lightweight test rig were able to quickly determine which impeller designs were unable to generate the necessary adhesion forces, not much more information could be gleaned from its use. For instance, although there were 4 impellers that had the ability to generate sufficient adhesion forces to hold the rig onto the underside of a table, it was impossible to objectively determine the relative performance between those 4. An instrumented test rig was constructed in order to measure the actual adhesion forces being generated.

The test rig consists of a wooden base, to which a lever and a Plexiglas plate are mounted (see Figure 7-12). The lever pivots about a horizontal axis, with the pivot point located in the middle of the lever, like a seesaw. The impeller is mounted onto the output shaft of the DC hobby motor, and the motor is mounted in a Plexiglas frame. That frame is hung from one end of the lever, such that it can swing freely. A $467 \mathrm{~g}$ counterbalance weight located on the other end of the lever is then adjusted so that it cancels out the weight of the Plexiglas frame and impeller, and so that the lever comes to rest in a horizontal orientation. 


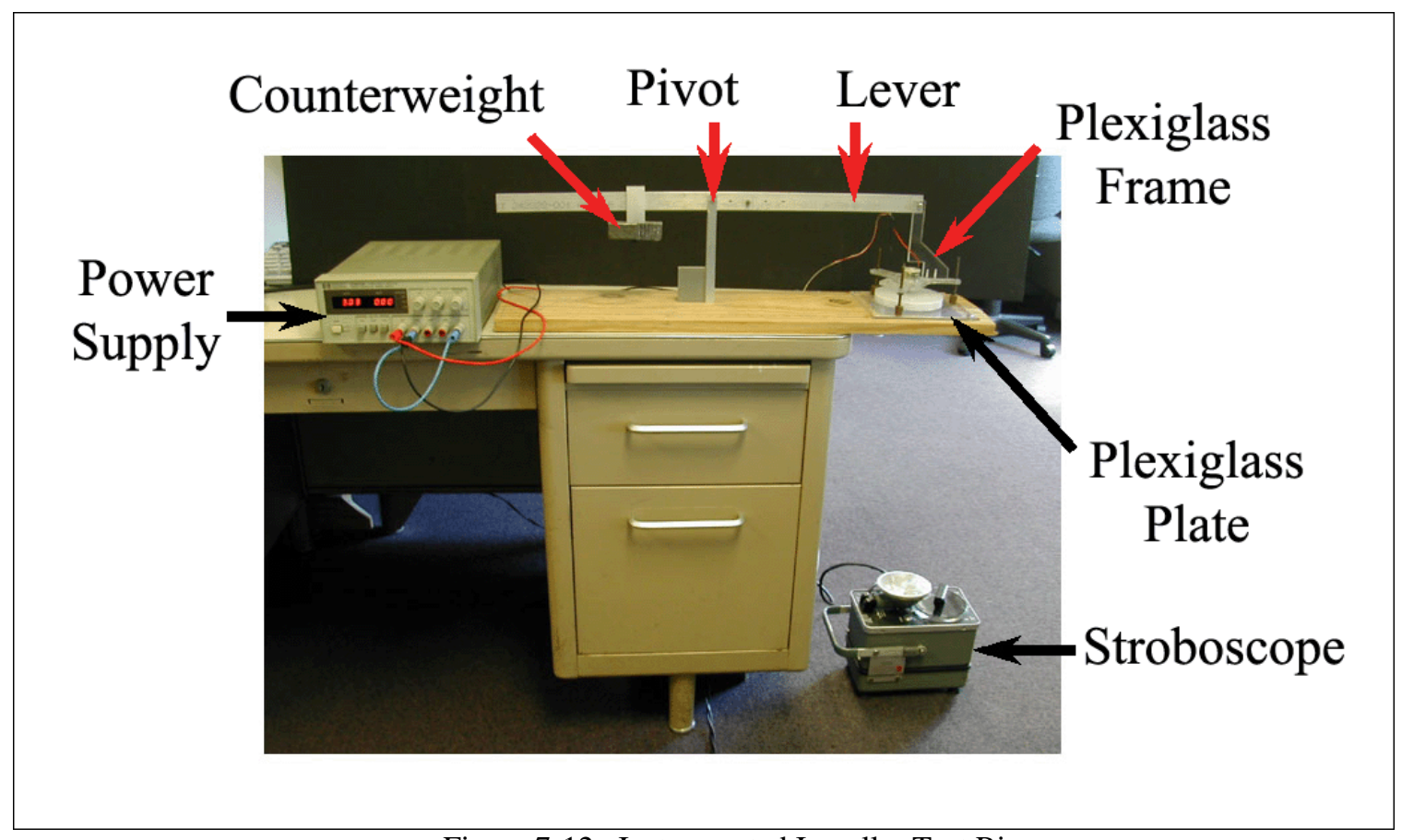

Figure 7-12. Instrumented Impeller Test Rig

The Plexiglas frame holding the hobby motor has three feet, whose purpose is to hold the impeller at a fixed, adjustable distance away from the substrate. The feet consist of threaded rod with rubber end caps to act as feet. The Plexiglas is threaded such that the length of the feet can be quickly and easily adjusted by turning the threaded rod. Jam nuts were used in order to prevent the threaded rod from turning during a test.

The Plexiglas frame was designed such that the axis of the hobby motor passes through the attachment point between the frame and the test rig lever. It was also designed such that its mass is balanced. As a result, when the frame is hung from the test rig's lever, the axis of the motor comes to rest in a vertical orientation. The total weight of the Plexiglas frame and hobby motor is $139 \mathrm{~g}$.

A Plexiglas plate is also mounted to the test rig at a location that coincides with a similar shaped hole in its wooden base. This is mounted in a horizontal orientation, directly below where impeller is hung. The Plexiglas plate has two purposes. It provides 
a completely flat, smooth surface for the impeller to act against, and it also allows the impeller to be viewed from above and beneath. The ability to view the impeller from underneath allows the angular velocity of the impeller to be measured using a Stroboscope (see Figure 7-13).

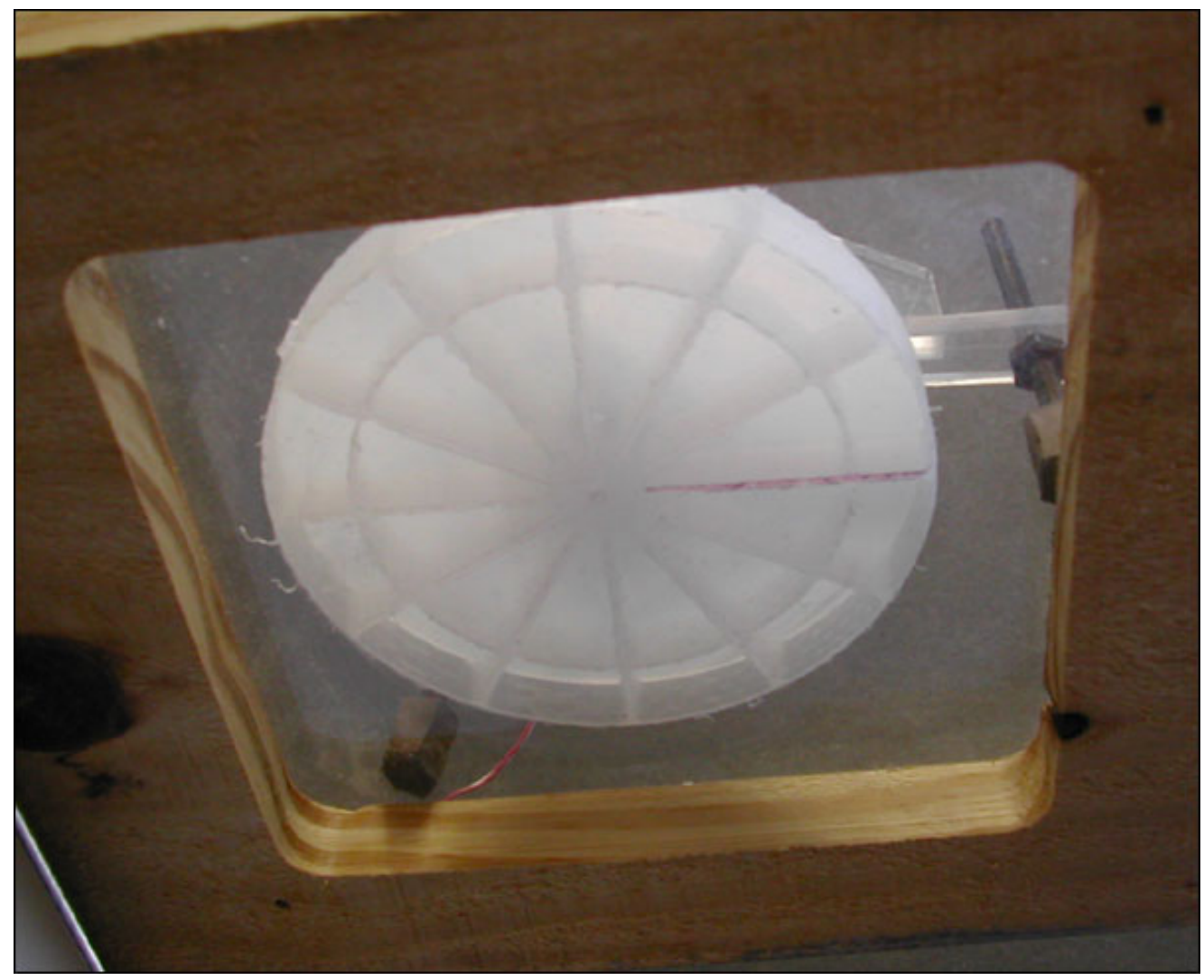

Figure 7-13. Plexiglas Plate from Underneath

Finally, a fish scale is attached to the test rig's lever. The adhesion force generated by the impeller pulls the lever in one direction. By pulling in the other direction with the fish scale, the adhesion force generated can be measured. 


\subsubsection{Test Procedure}

The instrumented test rig was clamped to a table to prevent it from moving during tests. The end of the test rig with the Plexiglas plate overhung the edge of the table, allowing the impeller to be viewed from above and beneath.

The feet of the Plexiglas frame were adjusted so that the impeller was located $1 \mathrm{~mm}$ away from the Plexiglas plate. The position of the counterbalance was adjusted to cancel out the weight of the Plexiglas frame and impeller, such that the entire assembly came to rest with the feet of the frame just touching the Plexiglas plate.

Power was provided to the hobby motor by a Tektronix PS280 DC Power Supply, and the voltage supplied to the motor was adjusted to change the motor speed. Measurements were taken when the impellers were spinning at 2000, 3000, 4000, 5000, and 6000 RPM, when possible.

A straight, radial line was drawn on a vane of each of the impellers, and the rotational speed was verified with a stroboscope (General Radio Company STROBOTAC Type 1531-A). A stroboscope emits pulses of light at specified intervals. The stroboscope was placed on the floor pointed upward, so that the angular speed could be determined without moving the impeller from the test configuration.

For instance, if the desired speed of the impeller were 3000RPM, the stroboscope would be adjusted to pulse 3000 times per minute. When the impeller reached the desired speed, it would make one revolution per pulse from the stroboscope. If there were no

other light source in the room, the impeller would then appear to be motionless under the light of the stroboscope. Once the impeller reached the desired speed, the stroboscope was set to pulse at twice the frequency, to verify that the impeller was not spinning at 
twice the desired speed. If the impeller were spinning at twice the desired speed, the impeller would again appear to be motionless. If the impeller were moving at the correct speed, the line drawn on the impeller would appear in two different places, because the impeller would only be able to make one-half rotation in between pulses from the stroboscope.

After the impeller speed was confirmed to be the desired speed, the voltage that the power supply was supplying was recorded.

The fish scale was then slowly pulled upward, which pulled the lever in the opposite direction of the adhesion force. The force that was required to pull the impeller away from the substrate was recorded for each of the specified speeds. The test was repeated a second time immediately afterward to verify the recorded numbers.

The impellers that had straight vanes were only spun clockwise (as viewed from below in the instrumented test rig), since they could be expected to generate the same adhesion force in either direction. The impellers with curved vanes were tested when spinning both clockwise and counter-clockwise.

Unfortunately, data was only taken on impellers \#1, 3, 4, and 5, because the DC hobby motor that was being used malfunctioned before the rest of the tests could be completed.

However, the data that had already been taken was sufficient to determine that the impellers being tested would not be able to generate enough force to hold up a robot. Since impellers \#1, 3, 4, and 5 had the highest adhesion performance with the lightweight test rig, the other impellers could not be expected to generate higher adhesion forces in the instrumented test rig, and therefore, data for the other 9 rotors was not necessary. 


\begin{tabular}{|c|c|c|c|c|c|}
\hline Impeller & 2000RPM & 3000RPM & 4000RPM & 5000RPM & 6000RPM \\
\hline $1(\mathrm{CW})$ & $0.2 \mathrm{~N}(1.8 \mathrm{~V})$ & $0.5 \mathrm{~N}(2.5 \mathrm{~V})$ & $0.9 \mathrm{~N}(3.5 \mathrm{~V})$ & $1.3 \mathrm{~N}(4.5 \mathrm{~V})$ & $1.9 \mathrm{~N}(6.1 \mathrm{~V})$ \\
\hline $1(\mathrm{CCW})$ & $0.2 \mathrm{~N}(1.8 \mathrm{~V})$ & $0.6 \mathrm{~N}(2.5 \mathrm{~V})$ & $0.8 \mathrm{~N}(3.6 \mathrm{~V})$ & $1.3 \mathrm{~N}(4.7 \mathrm{~V})$ & $1.9 \mathrm{~N}(6.0 \mathrm{~V})$ \\
\hline $3(\mathrm{CW})$ & $0.3 \mathrm{~N}(1.9 \mathrm{~V})$ & $0.6 \mathrm{~N}(3.1 \mathrm{~V})$ & $1.0 \mathrm{~N}(4.7 \mathrm{~V})$ & $1.6 \mathrm{~N}(6.7 \mathrm{~V})$ & \\
\hline 4(CW) & $0.3 \mathrm{~N}(1.7 \mathrm{~V})$ & $0.6 \mathrm{~N}(2.7 \mathrm{~V})$ & $1.0 \mathrm{~N}(4.5 \mathrm{~V})$ & $1.5 \mathrm{~N}(6.1 \mathrm{~V})$ & \\
\hline $4(\mathrm{CCW})$ & $0.3 \mathrm{~N}(1.9 \mathrm{~V})$ & $0.6 \mathrm{~N}(2.9 \mathrm{~V})$ & $1.0 \mathrm{~N}(4.3 \mathrm{~V})$ & & \\
\hline $5(\mathrm{CW})$ & $0.3 \mathrm{~N}(1.7 \mathrm{~V})$ & $0.7 \mathrm{~N}(2.7 \mathrm{~V})$ & $1.1 \mathrm{~N}(4.1 \mathrm{~V})$ & $1.6 \mathrm{~N}(5.4 \mathrm{~V})$ & \\
\hline
\end{tabular}

Table 7-2. Generated Adhesion Forces by Tested Impellers

The data that were recorded are shown in Table 7-2. The data included the adhesion force that each impeller was able to generate at the specified speeds (also plotted in Figure 7-14), as well as the voltage required to spin them at those speeds (also plotted in Figure 7-15). The force measurements were accurate to $+/-0.1 \mathrm{~N}$, while the voltage measurements were accurate to $+/-0.1 \mathrm{~V}$.

As expected, as the angular velocity of the impellers was increased, they generated higher adhesion forces. The slope of the force vs. velocity curve increases with velocity, which was expected from the theoretical calculations done in Figure 7-1.

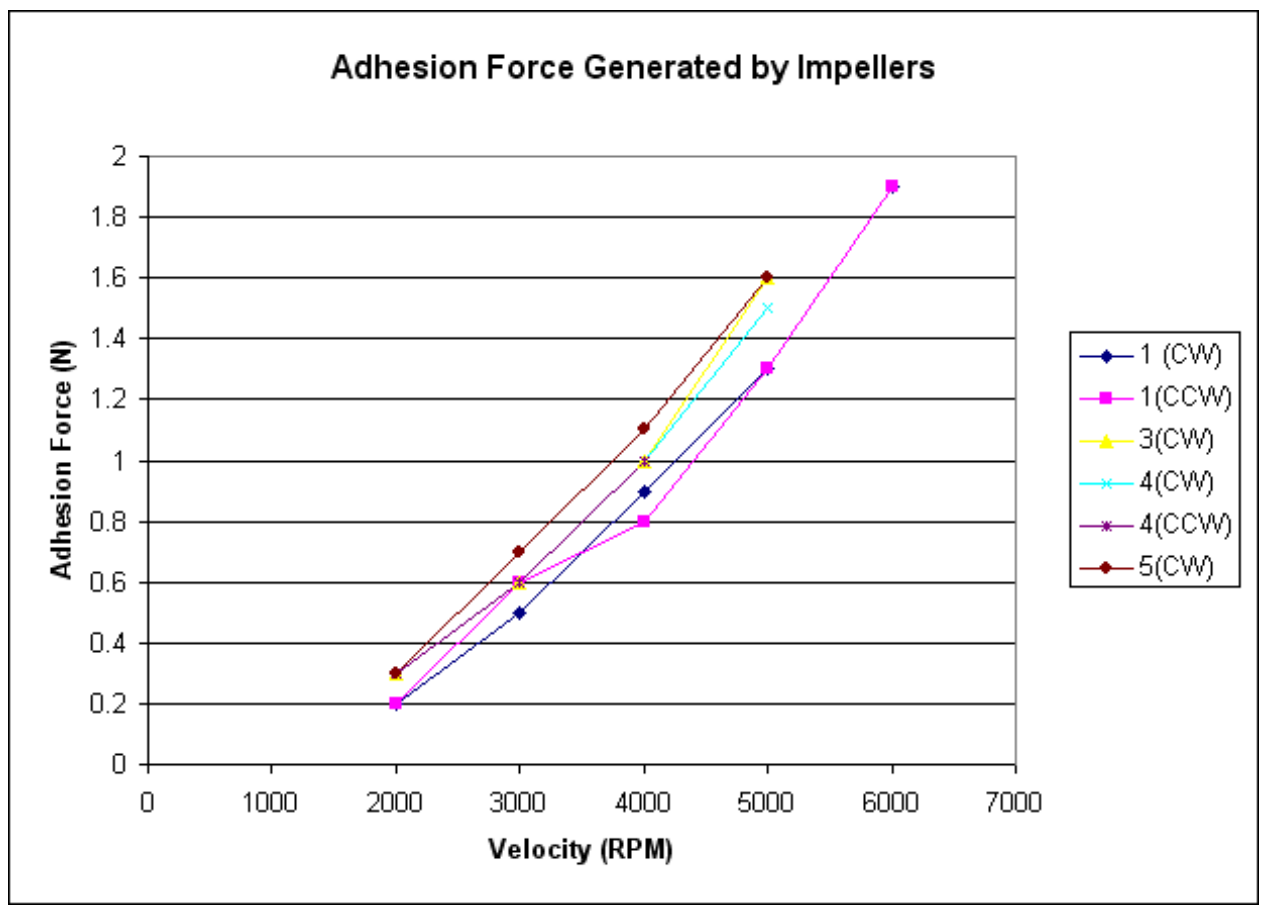

Figure 7-14. Adhesion Force Generated by Impellers 
Unfortunately, the measured adhesion forces were several times smaller than predicted by the theoretical calculations done in Figure 7-1. For instance, at 4000RPM, the angular velocity is $418.9 \mathrm{rad} / \mathrm{sec}$. A $10.16 \mathrm{~cm}$ impeller would have a radius of $0.0508 \mathrm{~m}$. The density of air is roughly $1.25 \mathrm{~kg} / \mathrm{m}^{3}$. From Figure $7-1$, the adhesion force generated by an impeller is

$$
\mathrm{F}=\pi \cdot \rho \cdot \Omega^{2} \cdot \mathrm{R}^{4} / 2
$$

Therefore, the impellers were predicted to create an adhesion force of $2.29 \mathrm{~N}$. This is much larger than the forces the impellers generated.

In order to generate sufficient forces necessary to be useful on a robot, the impellers would have had to be spun at a much higher angular velocity than that which the hobby motor was able to operate.

Impellers \#1 and \#4 were not symmetric, and as such, they were tested while rotating in both directions. However, there was very little difference in the adhesion force generated when those impellers were spun clockwise as opposed to counter-clockwise.

As expected, the measured adhesion forces were very similar to the total weight of the impeller and lightweight test rig, but not much more. It appeared unlikely that the impellers would be able to support a power-autonomous test rig onto the underside of a table. 


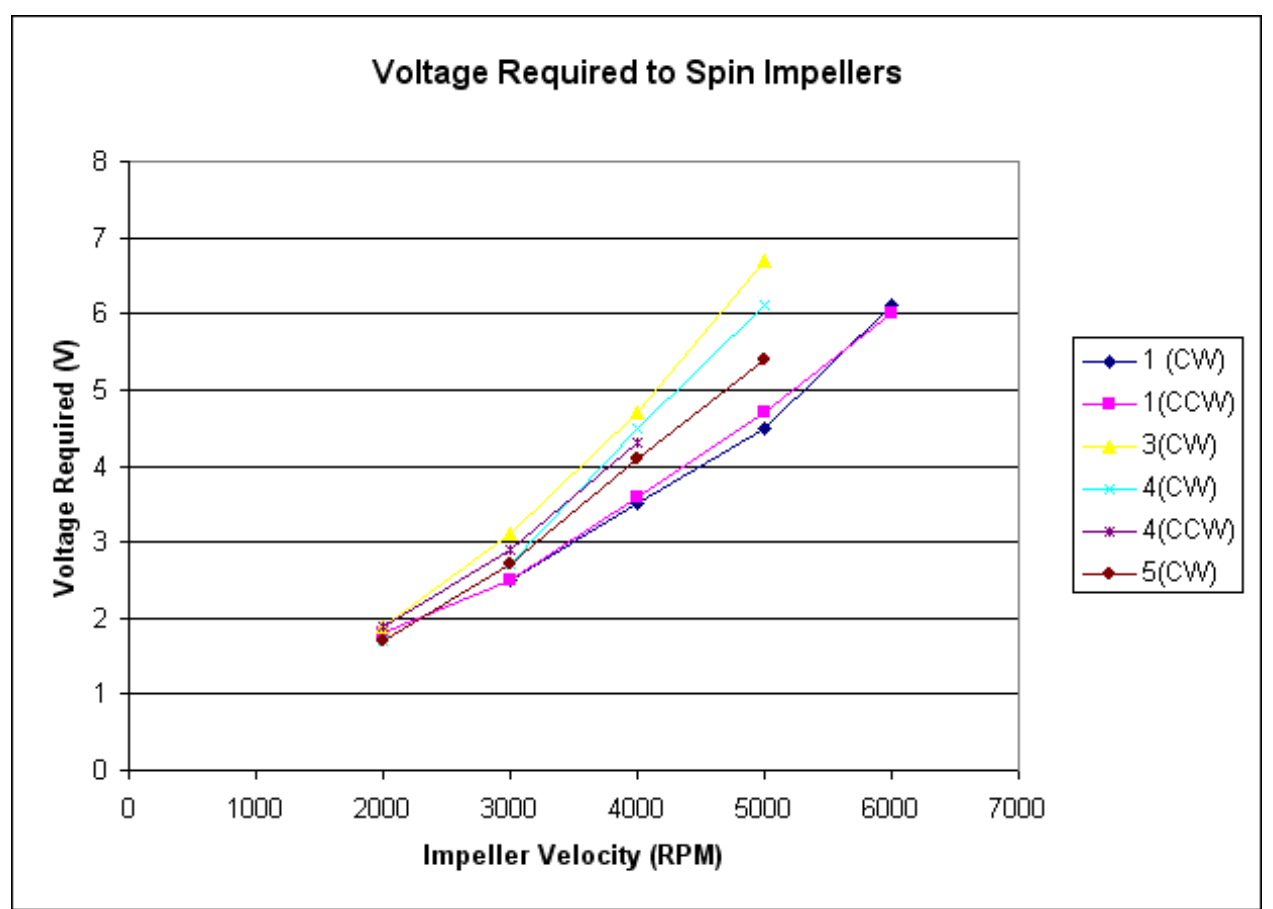

Figure 7-15. Voltage Required to Spin Impellers

Figure 7-15 shows the voltage required to get the hobby motor to spin the impellers at the specified speeds. The voltage increased with velocity similar to the adhesion forces. The increases in voltage were large, which was why no tests were attempted above 6000RPM. In order to generate necessary forces, a much higher performance motor would have been required.

Testing of impellers was halted because of their in ability to generate adhesion forces much larger than their total weight. Effort shifted to investigating a mechanical attachment method, which is described in Chapter 8. 


\section{Foot Morphology}

Four abstracted cockroach locomotion mechanisms were implemented in MechaRoach II. They included usage of features of leg morphology, leg compliance, body flexion, and gait adaptation. Foot morphology was originally intended to be the fifth abstracted cockroach locomotion mechanism. Cockroaches have several ways to grip substrates, including an actuated claw and spines on their tarsi [11]. These features can be seen in Figure 8-1.

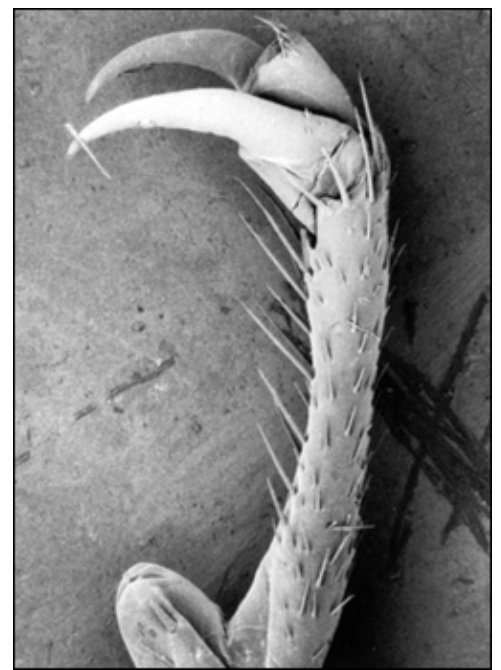

Figure 8-1. Cockroach Tarsus (Courtesy of Sasha Zill)

Cockroaches also use pads on their tarsi that allow them to grip smooth surfaces [18].

Actuated claws were designed to be mounted on the end of each 4-bar mechanism leg. The claws were spring-loaded to be normally closed, and were intended to be opened using tendons. The claw in a partially opened position can be seen in Figure 8-2. 


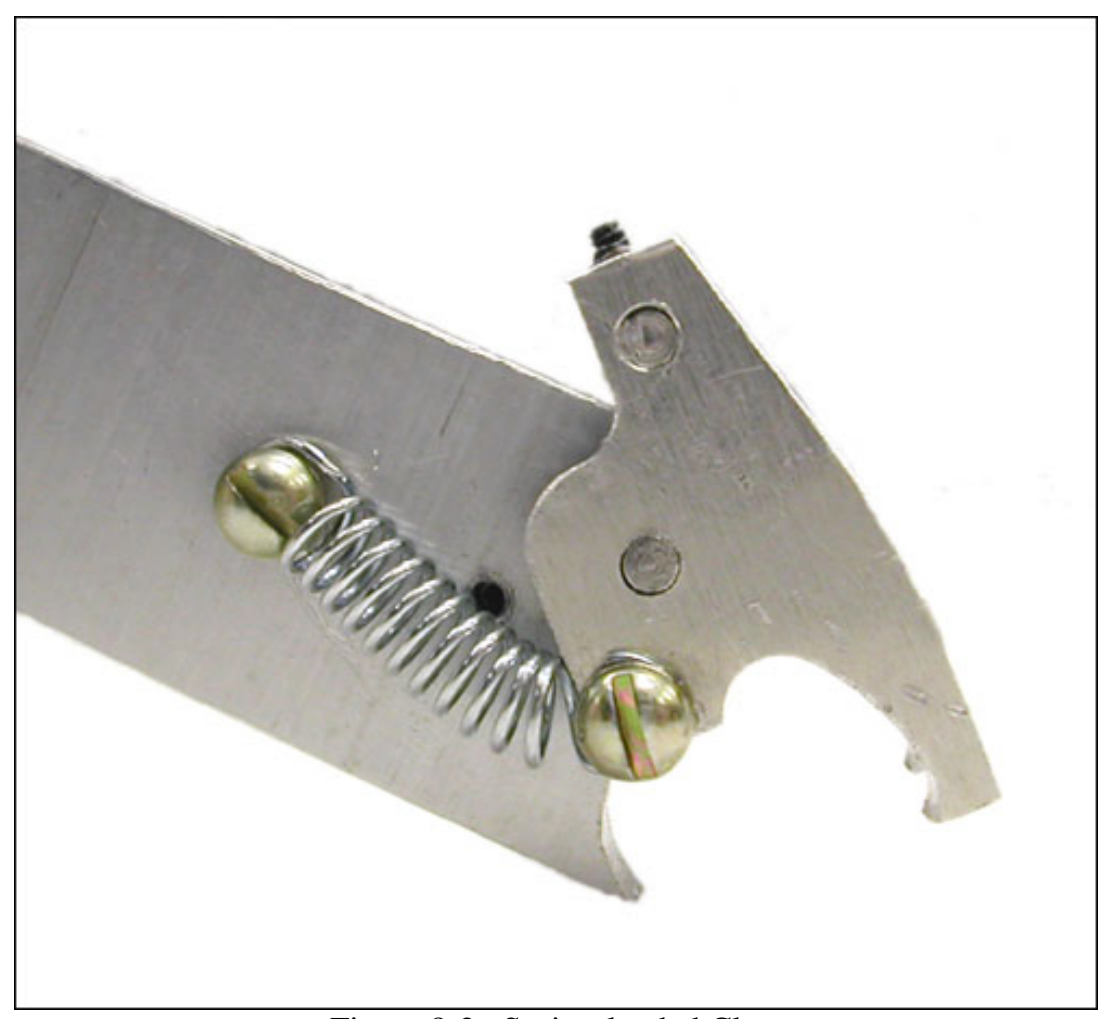

Figure 8-2. Spring-loaded Claw

The claw was designed to allow the robot to grasp and climb mesh or coarse substrates.

Among the challenges of designing a claw for use on a robot using 4-bar mechanisms as legs was designing the claws such that they could be smoothly engaged with the substrate at the beginning of stance, and disengaged at the beginning of swing. The opening of the claw had to be oriented in the correct direction, and care had to be taken to ensure that the claw was opened wide enough.

The claws were never fully implemented on MechaRoach II. During testing of leg designs, it had been determined that the leg mechanisms were not going to be able to allow the claws to be properly engaged and disengaged. The problem prevented the robot from making the transition between horizontal and sharply inclined surfaces, and is discussed in detail in Section 10.6. 


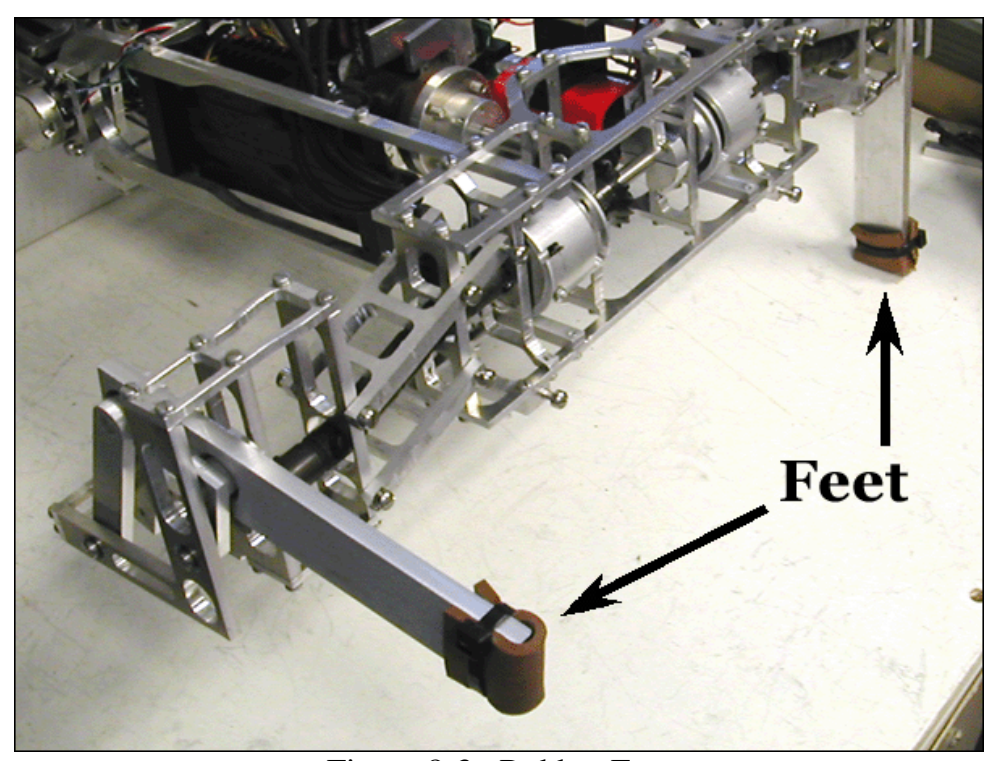

Figure 8-3. Rubber Feet

Instead of claws, rubber pads were wrapped around the end of the tibias to act as feet and give the robot traction (see Figure 8-3). Additional traction could have been created by increasing the contact area of the feet, and coating that surface with rubber. This modification was not performed. Since it had already been determined that regardless of foot design, the robot was going to be unable to make the transition between horizontal and sharply inclined surfaces, the potential gain from improved foot designs was minimal. 


\section{Robot Construction}

\subsection{Chassis}

The chassis was designed to be composed of a network of thin aluminum supports (see Figure 9-1). This composition allows the chassis to be relatively light. The chassis weighs $1.5 \mathrm{~kg}$, while the total weight of the robot is $7 \mathrm{~kg}$.
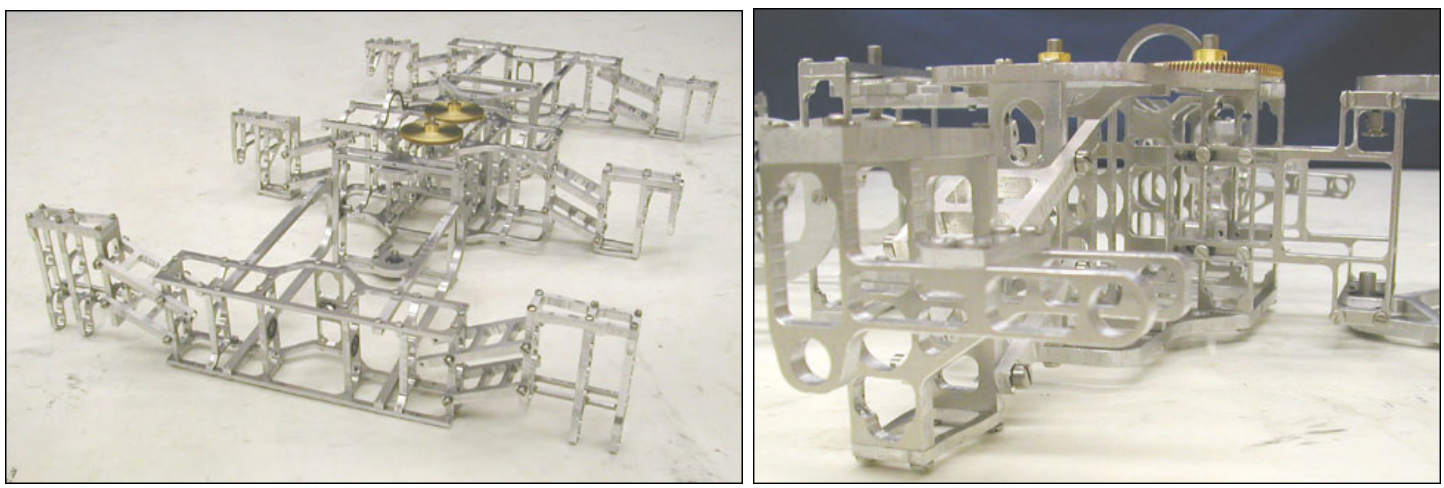

Figure 9-1. Bare Chassis of MechaRoach II

The parts that were assembled to form the chassis were machined from aluminum plate that were either $0.318 \mathrm{~cm}, 0.476 \mathrm{~cm}$, or $0.635 \mathrm{~cm}$ thick. The parts were first designed in Pro/ENGINEER, and then machined using a Hurco VM1 CNC milling machine. Figure 9-1 also shows two brass worm gears near the center of the robot that were part of the steering system, and were purchased from W.M. Berg, Inc.

The robot was designed to be easily serviced. This included dividing the chassis into sub-sections that could be easily detached from the rest of the chassis. Designing the chassis in this manner allowed broken or damaged parts to be replaced easily. It also allowed parts to be replaced quickly and easily if design changes were required.

Unfortunately, this design requires the use of over 400 fasteners to be used in the construction of the robot. These fasteners include cap screws, setscrews, and shoulder screws. All but 16 of those fasteners have English \#4-40 threads. The 16 that are not \#440 have English \#6-32 threads. The majority have the same threads so that only one 
screwdriver and one Allen wrench would be required to disassemble any part of the robot. Redundant fasteners are located in areas where one \#4-40 screw is not be able to support the load. No fasteners broke in service.

Unfortunately, the \#4-40 screws are too small to handle the amount of torque required to prevent them from working themselves loose during operation of the robot. As a result, the entire robot has to be checked regularly for loose fasteners. Almost all of the fasteners are equipped with lock rings, which reduces the rate at which the screws loosen themselves, but did not entirely prevent that from occurring. Leg compliance also helped reduce vibration transmitted to the chassis, which helps to prevent screws from loosening.

Larger fasteners that could handle more torque during installation would have fixed this problem. The sixteen \#6-32 screws installed on the robot never worked themselves loose.

\subsection{Steering}

Steering is accomplished in MechaRoach II through two body joints that are actuated by one motor (see Figure 9-2). The body joints enable the front and rear sections of the robot to pivot side to side. The front and rear sections can rotate 45 degrees to the left or right of center. Power is provided by a single 20-Watt 32mm DC Maxon motor that is capable of providing $41.7 \mathrm{mNm}$ of continuous torque output. The motor was designed to be provided with a 6-volt power source, but is provided with 7.2 volts in the robot. The motor weighs $240 \mathrm{~g}$, and is identical to the one that powers the body flexion joint. It is transversely mounted. 


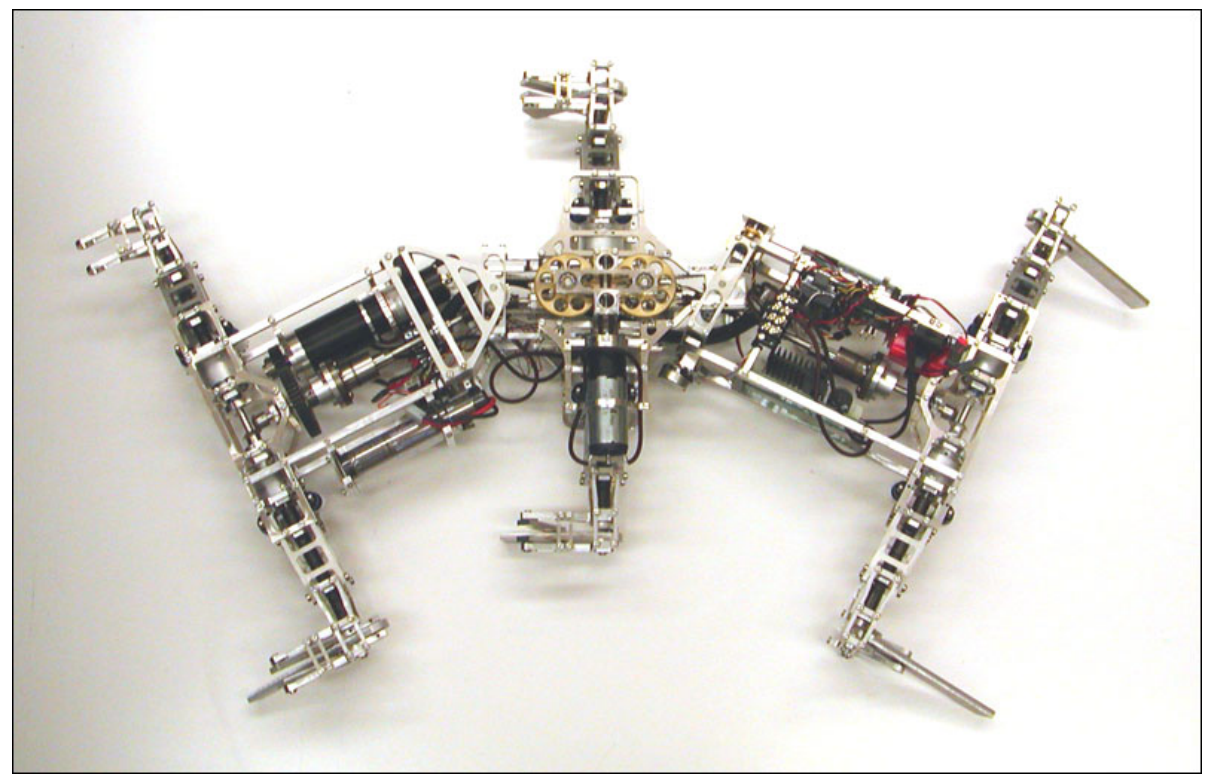

Figure 9-2. Body Steering Joints

A Maxon GP32 gearhead was mated to the motor, which provides a 3.7:1 gear reduction. It has a mass of $118 \mathrm{~g}$, and again is identical to the one powering the body flexion joint. An additional 50:1 gear reduction is provided by a worm gear/worm screw combination. A 100-tooth, 48-pitch worm gear with a $5.29 \mathrm{~cm}$ pitch diameter is connected to the front section of the robot. Another identical worm gear is connected to the rear section of the robot. Torque applied to the worm gear rotates the corresponding section of the robot left or right (see Figure 9-3). 


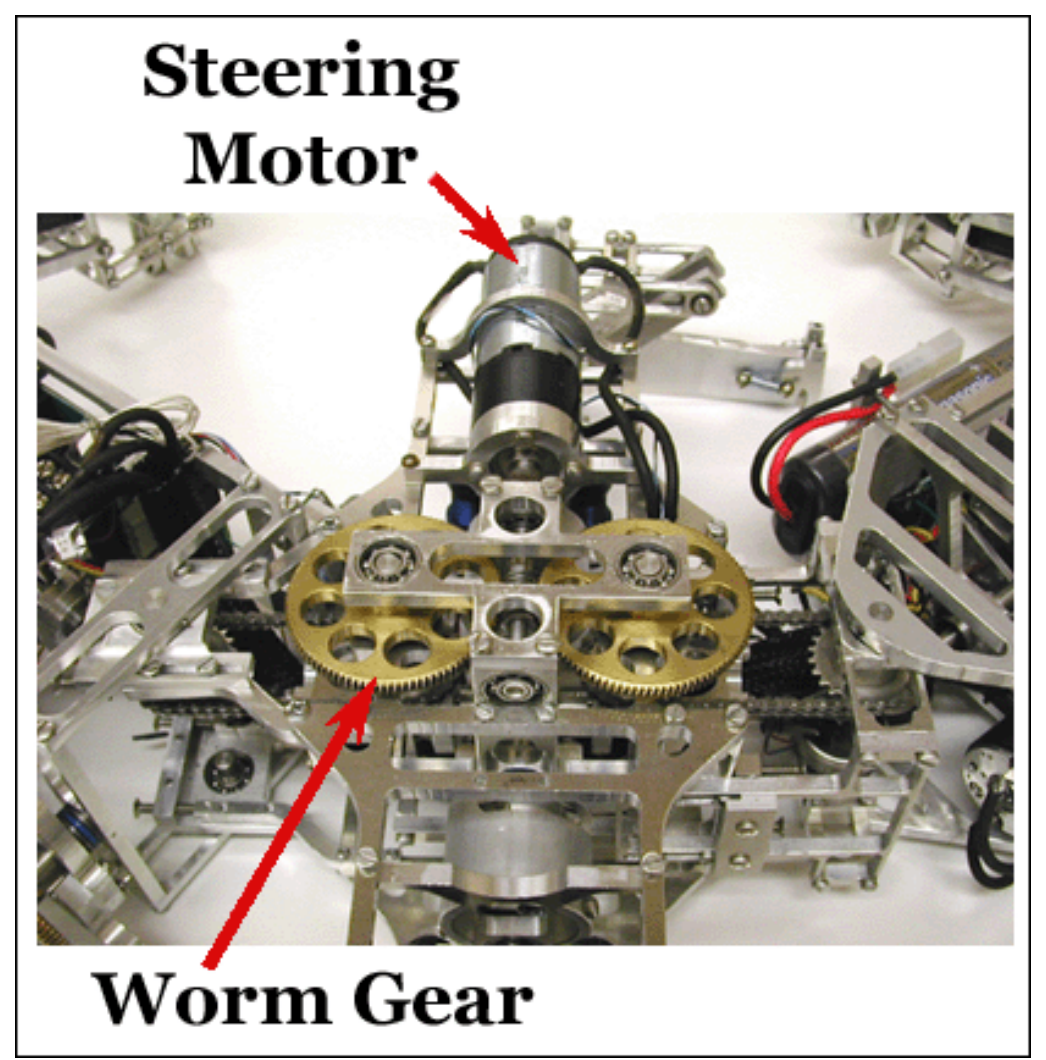

Figure 9-3. Transverse-mounted Steering Motor

Mounted onto the output shaft of the gearhead is a worm screw that meshed with the worm gears. A worm screw was used so that the steering system would be nonbackdrivable. If the system could be back-driven, power would have to constantly be supplied to the motor in order for it to maintain its position. With the worm screw, once the system has reached the desired position, very little power is required to keep it there.

Like the body flexion joint, the steering motor is turned into a servo with the use of a potentiometer and a Lynxmotion SHB-01 Speed Controller. Aluminum heat sinks had to be added to the speed controller to help dissipate heat.

The worm gears are coupled to the actual body steering joints through 0.1475in-pitch stainless steel miniature chain and 24-tooth chain sprockets.

The front body steering joint axis intersects the body flexion joint axis. The intersection was created so that the set of double universal joints that allow torque to be 
applied to the driveshafts through the body flexion joint would also allow torque to be transferred through the steering joint.

One alternative to body steering joints would have been automobile-like steering. Automobile-like steering would have involved tie-rods and steering joints located at each of the front and rear legs. There are two advantages to body steering. If automobile-like steering were used, the steered legs would have to be placed far from the body so that they would not interfere with the frame when turned. This would have the disadvantage of widening the robot. Another advantage of body steering was that if only the front and rear legs could reach footholds, the steering system could be used to change the position of the middle legs. This would allow the middle legs additional opportunities to gain a foothold while climbing. Automobile-like steering would not provide this capability.

The robot has a turning radius of $60 \mathrm{~cm}$ or $91 \%$ of body length on a horizontal surface.

Leg coordination is important to the steering system. When the legs do not operate in a tripod gait, turning is not possible. When not in a tripod gait, some legs are dragged across the ground in swing, which creates ground reaction forces that oppose turning.

\subsection{Powertrain}

MechaRoach II is propelled by a 90W 35mm DC Maxon motor that is capable of providing $77.7 \mathrm{mNm}$ of continuous torque. The motor weighs $340 \mathrm{~g}$, and was designed for a $15 \mathrm{~V}$ DC supply, but is provided with the same 7.2-volt supply that powers the steering and body flexion motors. The motor is mounted longitudinally in the rear section of the robot for weight distribution purposes. 
A Maxon GP42 gearhead was mated to the drive motor. The gearhead provides a 66:1 gear reduction and weighs $460 \mathrm{~g}$. An additional 2:1 gear reduction is provided by the spur gears that couple the gearhead to the rest of the robot's drivetrain. A 24-tooth, 20pitch pinion gear was mounted on the output shaft of the gearhead. This pinion gear meshes with a 48-tooth, 20-pitch spur gear that was mounted on a driveshaft that is connected to all of the legs. The resulting configuration could theoretically have supplied a maximum of $10.26 \mathrm{Nm}$ of continuous torque, if the associated geartrain losses were neglected. The drive motor is driven using a Devantech MD03 Motor Driver.

The cranks of the leg mechanisms are $2.54 \mathrm{~cm}$ long. If the motor and geartrain provide 10.26 Nm of torque, the endpoints of the cranks provide a $404 \mathrm{~N}$ force. The robot's front legs reach the lowest mechanical advantage value (0.298) of any of the legs during stance. Even with that mechanical advantage, the output force is still over $120 \mathrm{~N}$. The robot's total weight is $7 \mathrm{~kg}$, so only $68.6 \mathrm{~N}$ are required to propel it forward if it is walking straight up a vertical surface. These calculations neglect drivetrain losses, but show the torque provided by the drive motor is adequate. The mechanical advantage of the middle and rear legs are higher.

Torque is distributed to the legs through driveshafts and bevel gears. Universal joints are used to transfer torque through body joints. Usage of chains to transfer torque between different sections of the robot was considered. However, drive-chains would have resulted in leg coordination changes when the body joint was flexed. 


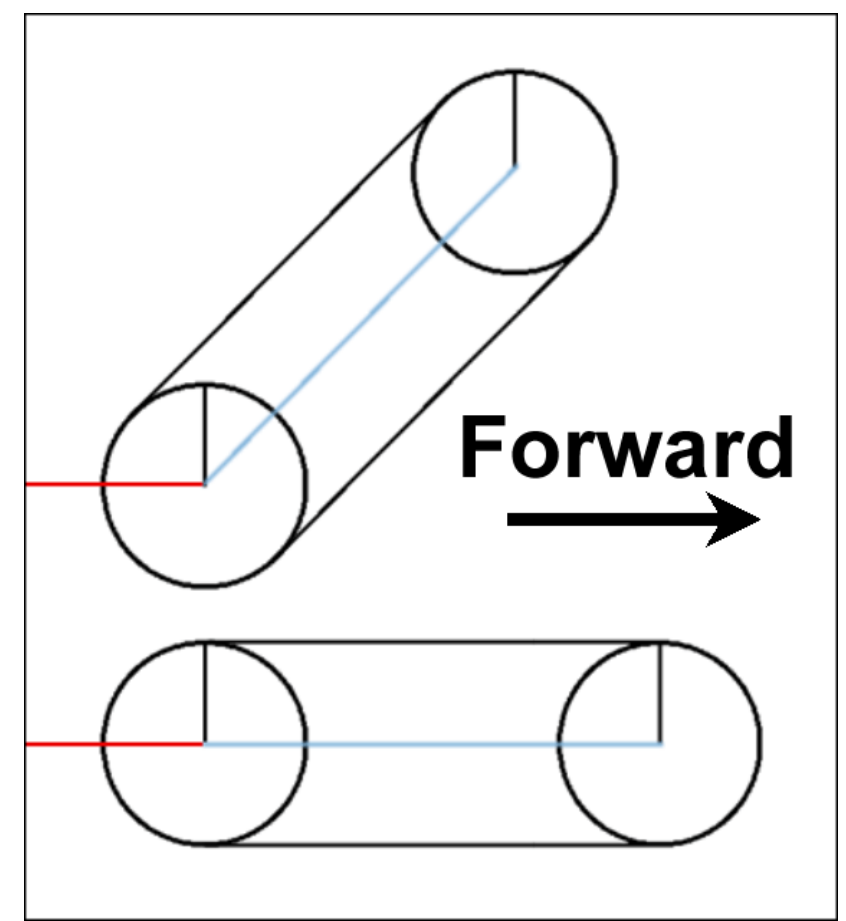

Figure 9-4. Drivechains Cause Changes in Leg Coordination

The problem with using drive-chains is illustrated in Figure 9-4. The red line represents the rear section of the robot. The blue line represents the front section of the robot, which is rotated upward or downward by the body flexion joint. When the front and rear sections are aligned with each other, as they are in the bottom of the figure, the front and middle driveshafts are in phase. When the body flexion joint is used to rotate the front of the robot upward, the drive-chain causes the front driveshaft to rotate relative to that section of the robot. The relative rotation of the front driveshaft is what causes changes to leg coordination.

Changes to the leg coordination would pull the robot out of an alternating tripod gait. Without a proper tripod of support, the robot would not be able to walk properly, and it was thought that this would prevent the robot from making transitions between horizontal and inclined surfaces. 
During the leg design process, the coordination of the legs was deliberately changed to determine if the robot could walk without the legs moving in a tripod gait. When the coordination was altered, the robot could not walk properly. In some circumstances, the robot could make forward progress, but dragged the feet in swing across the floor. In addition to preventing obstacle climbing, this also prevented the robot from being able to turn.

The usage of driveshafts ensured that the leg coordination would not change when the body flexion joint was activated, and the robot would always walk in an alternating tripod gait.

The usage of driveshafts also made the body steering joint system possible. If drivechains had been used, body joints that rotate the front and rear segments of the robot side to side could not have been implemented.

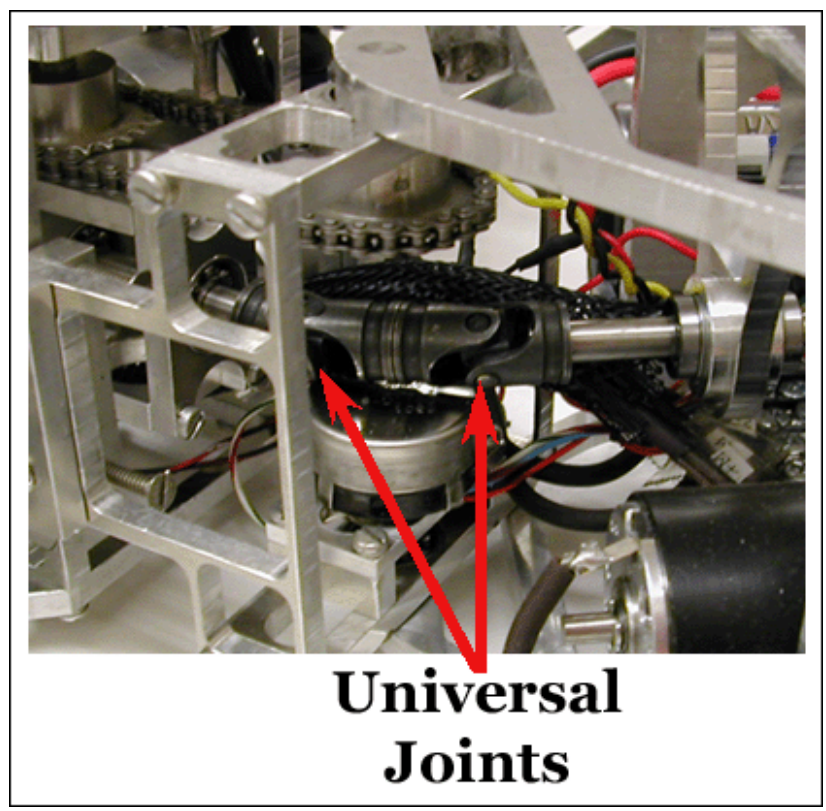

Figure 9-5. Double Universal Joints

Driveshafts require the use of universal joints to transfer torque between different sections of the robot that were separated by body joints (see Figure 9-5). Double 
universal joints are used to increase the relative rotation that can occur between the different sections of the robot. Unfortunately, double universal joints also cause longitudinal movement of driveshafts with the activation of body joints. This effect is illustrated in Figure 9-6.

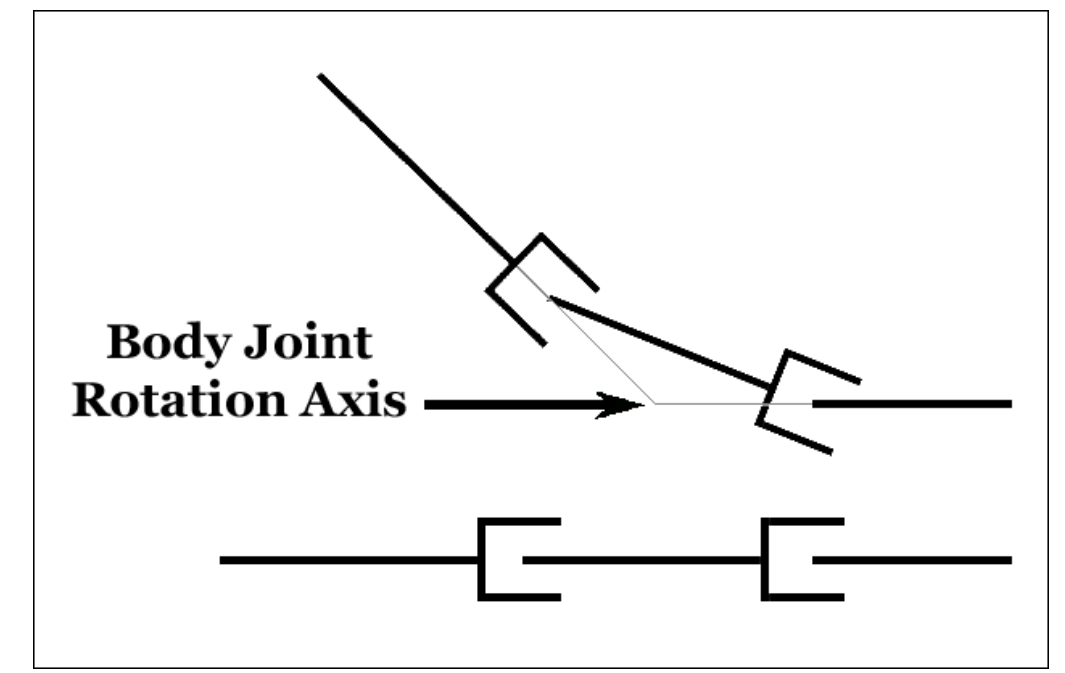

Figure 9-6. Double Universal Joints Cause Driveshaft Longitudinal Movement

In the lower part of Figure 9-6 is a drawing of the universal joints when the front and middle sections of the robot are aligned. Torque is delivered to one end of the double universal joints. It is transferred through one universal joint into an intermediate shaft before being transferred through the other universal joint.

When a body joint is flexed, the double universal joints move to the position shown in the upper part of the figure. Since the shaft between the two universal joints does not go through the axis of rotation, longitudinal movement of the driveshafts relative to the robot must occur.

The driveshafts in the front and rear sections of the robot transfer torque through ballsplines. Figure 9-7 shows the placement of the rear ball-spline in the robot. Ball-splines provide a telescoping joint to a shaft. Torque can still be transferred, but small longitudinal movements of the driveshafts are allowed to occur. 


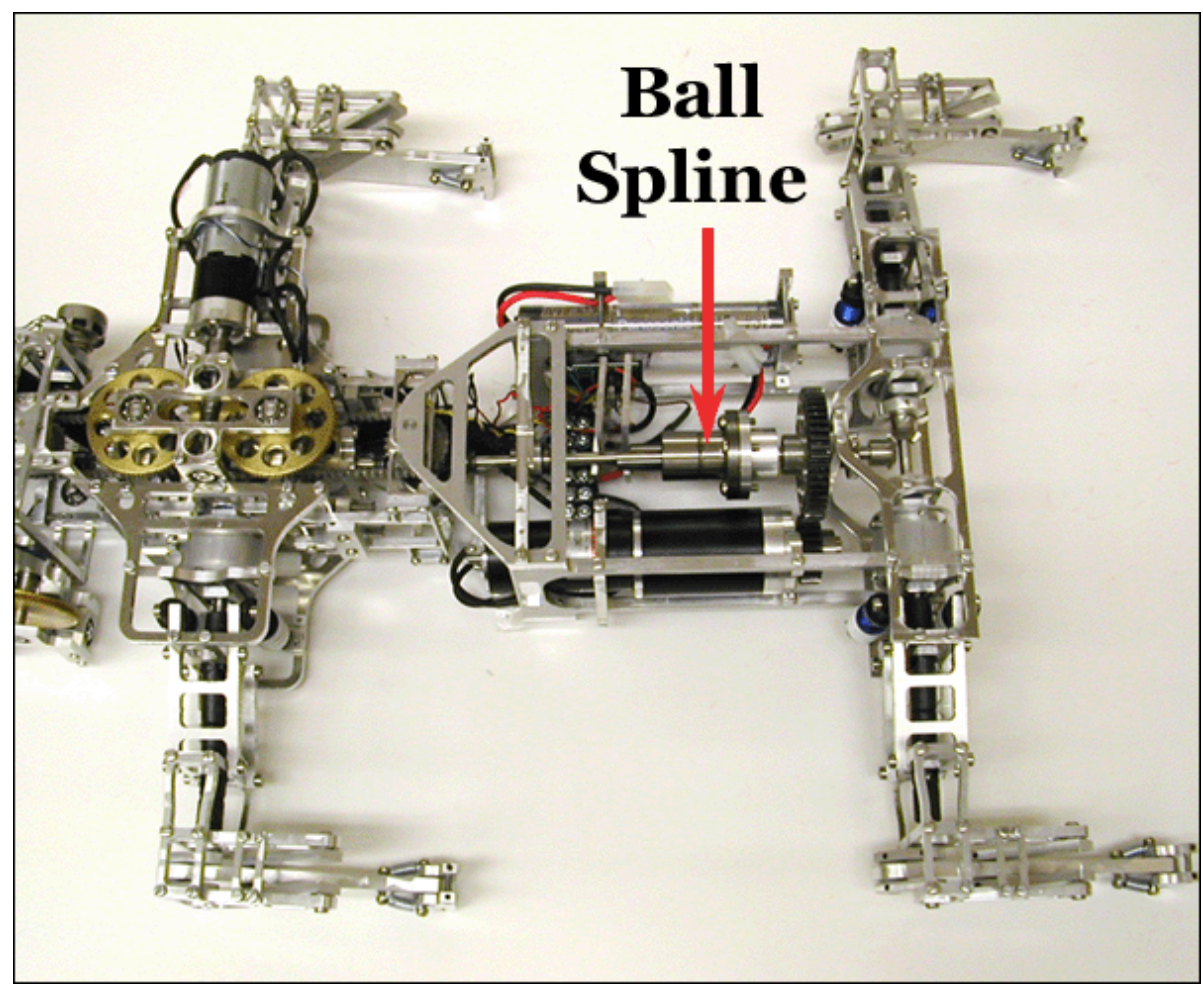

Figure 9-7. Ball-spline in Rear Section of Robot

Each ball-spline consists of two major components. One part is a flanged bearing. The other is a 13mm splined shaft. Small amounts of axial movement are made possible by the flanged bearing and splined shaft combination, but torque can be transferred between them. The splined shaft is mounted to a $0.635 \mathrm{~cm}$ diameter ground shaft, which is supported by a linear bearing.

The ball-splines are mounted longitudinally, while the driveshafts powering the legs are mounted transversely. 24-tooth, 16-pitch bevel gears were used to couple these driveshafts. Transverse driveshafts are prevented from moving axially with thrust bearings and custom-made shaft collars.

\subsection{Weight}

The typical Blaberus discoidalis is about $4.4 \mathrm{~cm}$ long and $2.25 \mathrm{~g}$ in mass [21]. At $66 \mathrm{~cm}$, MechaRoach was about fifteen times longer. Because the mass should increase 
with the cube of the length, a cockroach scaled up fifteen times should weigh about 7.59 kg. Since the robot weighed about $7 \mathrm{~kg}$, it is roughly proportional in weight to the cockroach.

During the design phase, special attention was paid to balancing the weight distribution. For instance, care was taken to distribute mass on the left and right sides such that the center of mass was kept on the midline of the robot. If the mass were distributed unevenly, legs on one side of the robot would be supporting more weight than ones on the other side. In addition, an unequal lateral distribution of mass might cause the robot to rotate in the coronal plane while climbing. To verify that the mass was evenly distributed left to right, the robot was suspended from a point along the midline of the chassis, to observe whether it leaned to one side or the other, and it did not.

The mass was also carefully distributed in the front and rear sections of the robot. If too much mass were located in the front of the robot, the center of gravity would lie in front of the middle legs. If the body flexion joint were used to rotate the front of the robot upward, the robot would be unable to remain standing on the middle and rear legs on a horizontal surface if the center of gravity were in front of the middle legs. The center of gravity ended up being located just behind the middle legs.

Mass distribution was balanced by placing components carefully (see Figure 9-8). The drive motor and body flexion joint motor were mounted longitudinally in the chassis, which also helped to distribute the mass more evenly. Electronics were distributed fairly evenly between the front and the rear of the robot, and the main battery pack was located in the rear of the robot, mounted longitudinally. 


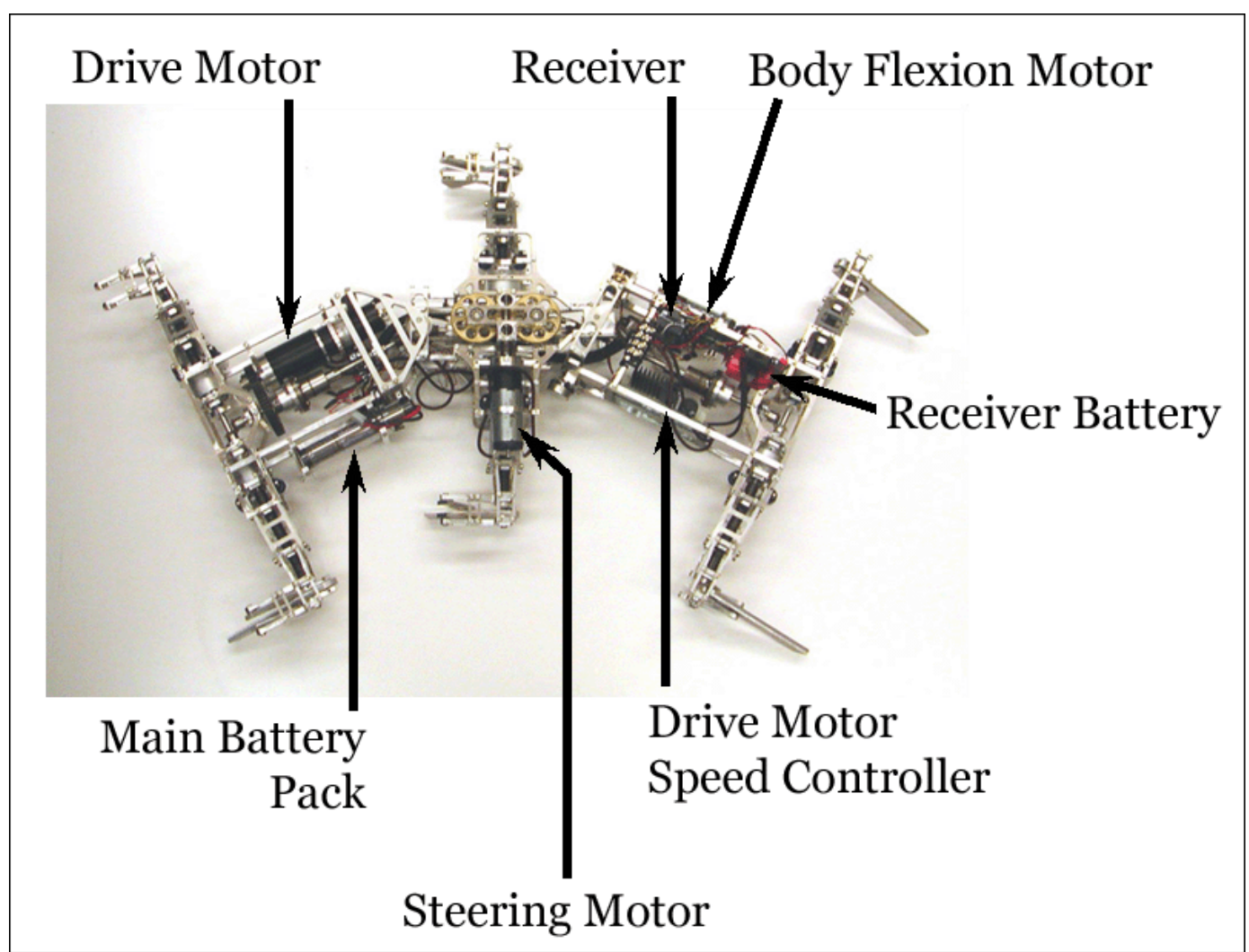

Figure 9-8. Distribution of Mass about the Robot

Unfortunately, even though the center of gravity ended up being located in the desired location, the mass was concentrated in the center section of the robot. This is undesirable, because the middle legs are required to support more of the robot's weight than the front and rear legs. The components that caused this concentration of mass included the steering motor, and several steel components such as bevel gears. The mass could not be redistributed without a major redesign of the chassis, so redistribution was never completed. The components causing the problem also could not be easily reduced in weight. 


\section{Results and Discussion}

10.1. Walking Speed

The robot is able to walk on a horizontal surface at a rate of about 1/18 body length/sec $(3.6 \mathrm{~cm} / \mathrm{sec})$. This rate was determined by measuring the amount of time the robot required to travel one body length with a running start. The robot required about 18 seconds to travel $66 \mathrm{~cm}$, and the motor was being driven at its top speed during the test. With a different gear reduction, the robot would have been able to walk faster and still maintain stable locomotion. A faster walking speed could also have been achieved by supplying the drive motor with its nominal voltage.

Walking speed was not an important consideration in the design of the robot. It was more important to create a robot that would have enough power to climb inclined surfaces. Another important goal was to design the powertrain such that the robot could be operated at a range of speeds. Poor mechanical advantage characteristics and improper gearing prevented MechaRoach I from being operated at a range of speeds. MechaRoach I could only move in a stable gait if it were driven at its top speed.

While the top speed of MechaRoach II is around 1/18 body lengths/sec, the robot is typically operated at speeds much slower. Operation at lower speeds allows the operator to stop the robot in time if a mechanical failure is imminent. Lower speeds also allow for more careful observation of the robot's behavior.

Because the robot was almost always operated at a speed below its maximum, it can be concluded that the top speed was adequate for the purposes of testing strategies for transitioning between horizontal and inclined surfaces. Higher speeds would be 
beneficial if the robot were intended for applications where it would be limited to walking on horizontal surfaces.

\subsection{Horizontal Foot Forces}

Earlier, it was explained that the front legs of the cockroach create decelerating forces, the rear legs create accelerating forces, and the middle legs create both during walking and running. Forces generated during the interaction of the feet of the robot with the ground were never directly measured, because the appropriate equipment was not available. However, tests were conducted in which the robot was operated on surfaces where little to no traction could be found. The results of these tests can be used to infer the direction of the horizontal foot forces.

Immediately after heel strike, the front legs slid forward on the slippery surface. Then, towards the end of stance, the front legs slid backward. This suggests that the front legs of the robot began stance creating decelerating forces, and finished stance creating accelerating forces in the horizontal direction. Accelerating and decelerating forces were created, despite the front foot always being in front of the shoulder during stance, which put those legs in a decelerating orientation.

The middle and rear legs, in contrast, did not slide forward after heel strike, but always slid backwards towards the end of stance on a slippery surface. The direction of sliding suggests that the middle and rear legs always create accelerating forces in the horizontal direction.

The discrepancy between the directions of the horizontal foot forces may have to do with the robot's velocity. At the robot's maximum speed (1/18 body lengths/sec), the 
robot moved a lot slower than a cockroach, relative to its size. Blaberus discoidalis cockroaches have been recorded running at speeds of up to 10 body lengths/sec [43]. It is possible that if the robot had been moving at a higher rate of speed, the direction of the horizontal foot forces would have been different.

When faced with a slippery surface, the feet of Whegs $^{\mathrm{TM}}$ robots always slide backwards, which implies that all of the feet are creating accelerating forces in the horizontal direction. By only creating accelerating forces, those robots are able to climb well even when many of their legs are not able to touch the ground.

In contrast, the front legs of MechaRoach II begin stance by creating decelerating forces. As a result, the robot had a much harder time climbing when the middle legs could not touch the ground and the robot depended on the front legs to pull the robot forward.

\subsection{Torsional Compliance}

The passive torsionally compliant devices operated as expected. Movement was smooth between the input and output shafts, and the design was simple and light. The devices properly allow 180 degrees of relative rotation between the input and output shafts.

The passive torsionally compliant devices are activated even during walking on a horizontal surface. After heel strike, the legs dwell while the springs in the devices are wound up. The leg then proceeds to move the foot in the rearward direction until toe off. After toe off, the spring in the torsionally compliant device unwinds, and rapidly moves the leg back towards the heel strike position. These devices allow the output shaft to 
rotate 180 degrees independently of the input shaft. Because the middle legs support more weight than the front and rear legs during horizontal locomotion, they almost completely wind and unwind the torsionally compliant devices during each swing-stance cycle. The full 180-degree rotation that is allowed between the input and output shafts occurs in the front and rear legs only during climbing.

Activation of the torsionally compliant devices during walking extends the amount of time each leg spends in stance, and reduces the amount of time that they spent in swing. Extending the length of stance causes more overlap to occur between the two tripods of support in an alternating tripod gait. More overlap between the periods when both tripods are in stance means that there is less time when only three legs are supporting the weight of the robot. Having more legs on the ground adds to the stability of the robot during standing and walking. The torsionally compliant devices do not affect the overall speed of the robot during horizontal locomotion.

A running start was required when measuring the robot's speed because the robot takes a few steps before reaching a repeating gait. In the initial steps, the passive torsionally compliant devices do not get wound up as much as they do when the gait reaches steady state. During these initial steps, the robot's forward speed is slower.

In order to quantify the effect that the torsional compliance devices had on walking, various tests were performed. In one test, pins were inserted to lock the outer housing and the rotor of the torsionally compliant devices together. This modification effectively eliminated torsional compliance, and the robot was no longer capable of gait adaptation. Adjustments were made to the timing of each leg's stance-swing cycle such that the robot would walk in an alternating tripod gait. During this test, the robot was made to walk 
across a horizontal surface. The robot was able to make forward progress, but much more body roll was seen as the load was transferred from one tripod of support to the other. In addition, the robot could barely lift the middle legs off the ground in swing.

When the body flexion joint was used to raise the front of the robot up, the middle legs were no longer able to get off the ground during swing, and the robot could not make any forward progress. The middle legs were 180 degrees out of phase, and while one was pushing backward in stance, the other leg was pushing forward because it was supposed to be in swing. The middle legs were supporting the majority of the weight of the robot, which caused those legs to generate the most traction. As a result, the robot rotated back and forth in the coronal plane, while the rear legs were dragged side to side.

The passive torsionally compliant devices allow each leg to extend its stance phase while shortening its swing phase. Changing the ratio between the time spent in stance and swing allow each leg to support the weight of the robot for a higher percentage of the time. This stabilizes the robot because the weight is distributed between more legs at a time. When the torsional compliance is removed, the weight is distributed only among the three legs of the tripod in stance, causing more body roll to occur. Analysis of video of the robot walking was used to determine how much the torsionally compliant devices changed the ratio between the time spent in stance and swing.

Video was analyzed with the robot walking at a constant speed in three different configurations. In the first configuration, torsional compliance was locked out, and all six legs were allowed to touch the ground. In the second configuration, the torsionally compliant devices were allowed to operate normally, and all six legs were able to touch the ground. In the third test, torsional compliance was allowed and the body flexion joint 
was used to lift the front of the robot up, preventing the front legs from touching the ground. The tests in which only four legs could touch the ground were performed to determine if the different loading conditions affected the percentage of the time that each leg spent in stance.

After the robot reached a steady-state gait in the video, one step was analyzed frame by frame. The number of frames that each leg spent in stance were counted, and compared to the number of total frames that each leg took to complete one swing-stance cycle. The video camera recorded 30 frames/second.

The data observed from video were compared to theoretical data taken from Section 3.7. The data came from the Mechanism Analyzer program described in Section 3.5.4, which predicted the amount of time that each leg would spend in swing and stance if the robot did not have torsionally compliant mechanisms.

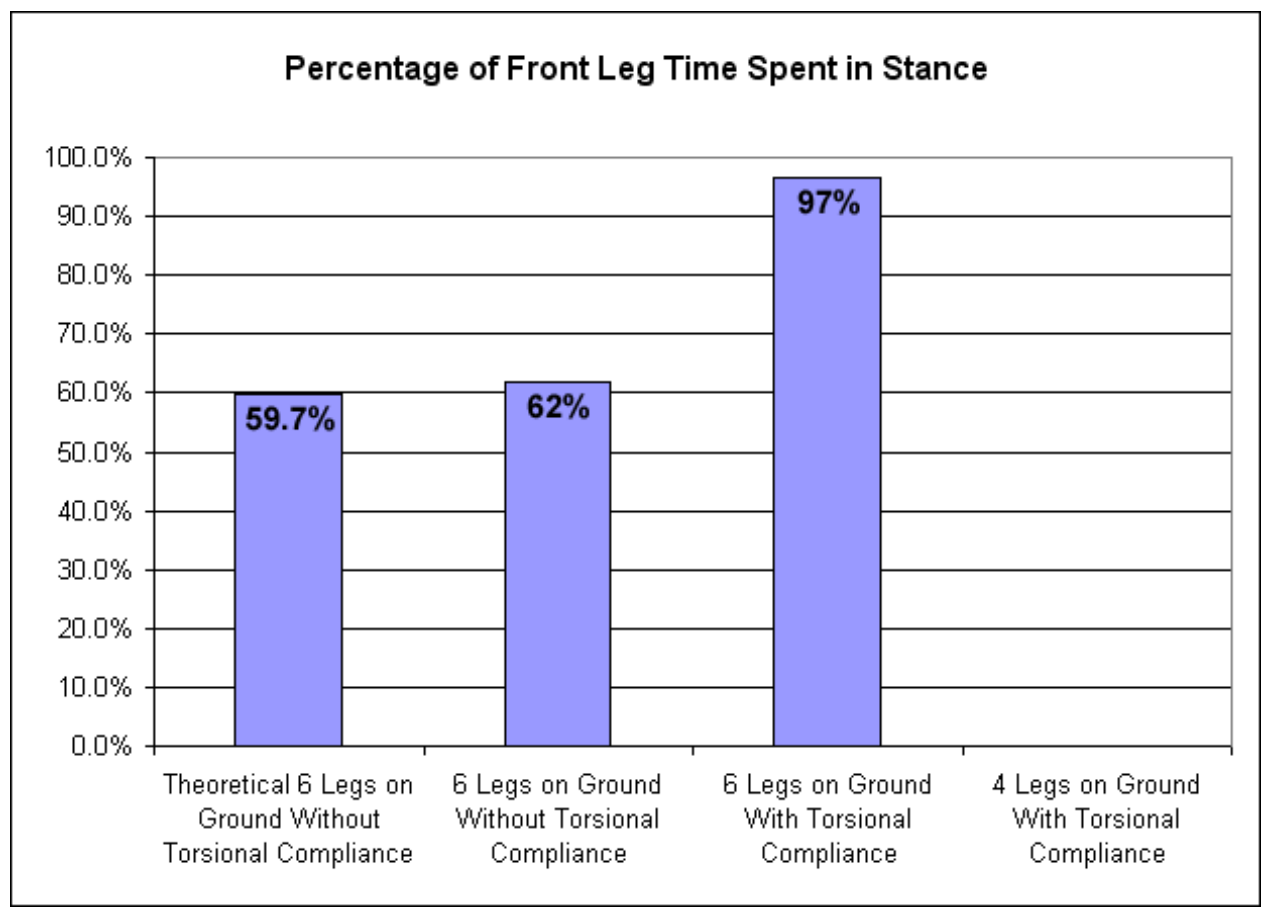

Figure 10-1. Percentage of Front Leg Time Spent in Stance 
Figure 10-1 shows the percentage of the time that a front leg spent in stance for the three tests, plus the theoretical prediction from the Mechanism Analyzer program. Earlier it had been explained that the transition between swing and stance for the front legs had been defined as the point where the vertical velocity of the front foot changed from being positive to negative, because the front leg might strike an obstacle while the foot was being swept downward and the front leg was able to support the robot if that occurred.

Using this definition of stance, the first column of the figure shows that use of the program predicted the front leg would spend $59.7 \%$ of each cycle in stance if torsionally compliant devices were not used. When the robot was tested without torsionally compliant devices, the front leg spent $62 \%$ of each cycle in stance. When torsional compliance was allowed, the front leg spent $97 \%$ of its time in stance. The torsionally compliant devices were able to dramatically prolong the stance phase of the front legs.

The final column of the figure is empty, because the front legs were lifted off the ground by the body flexion joint in that test. 


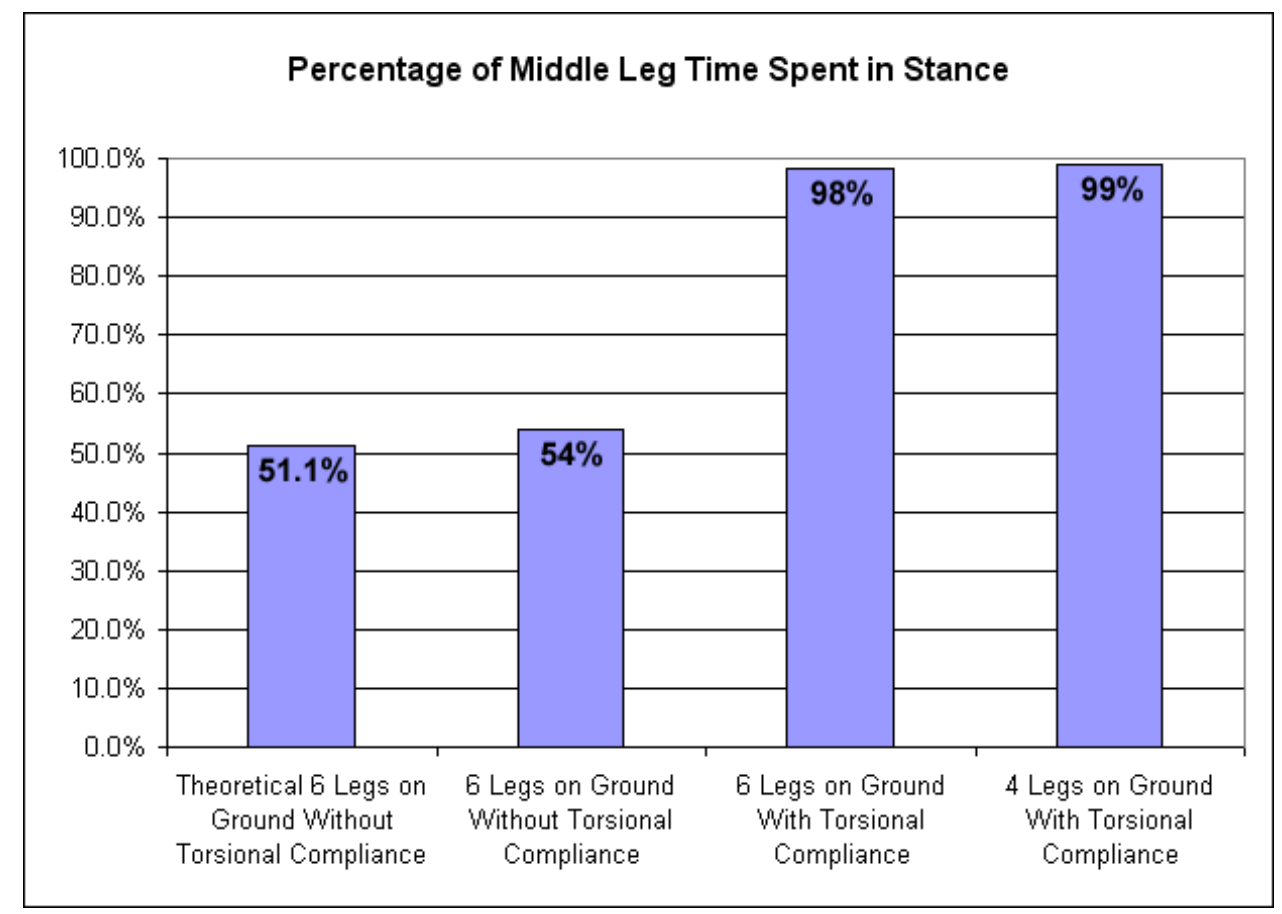

Figure 10-2. Percentage of Middle Leg Time Spent in Stance

Figure 10-2 shows the percentage of the time a middle leg spent in stance for the three tests, where stance was defined as any time during which the foot was touching the ground. The figure also shows the prediction from the Mechanism Analyzer program in the first column.

Usage of the program predicted the middle leg would spend 51.1\% of each cycle in stance if torsional compliance was not available. When the torsionally compliant devices on the robot were locked and the robot walked on 6 legs, the middle legs spent $54 \%$ of their time in stance. When torsional compliance was available, the percentage of time spent in stance increased to 98\%. Finally, with the body joint lifting the front section of the robot off the ground, the middle legs spent $99 \%$ of their time in stance. In that test, the middle legs were heavily loaded, because they were supporting the weight of the front section of the robot, in addition to the middle section. As a result, 100 video frames of 
that cycle were spent with the middle leg in stance, and only one frame showed the middle leg in swing.

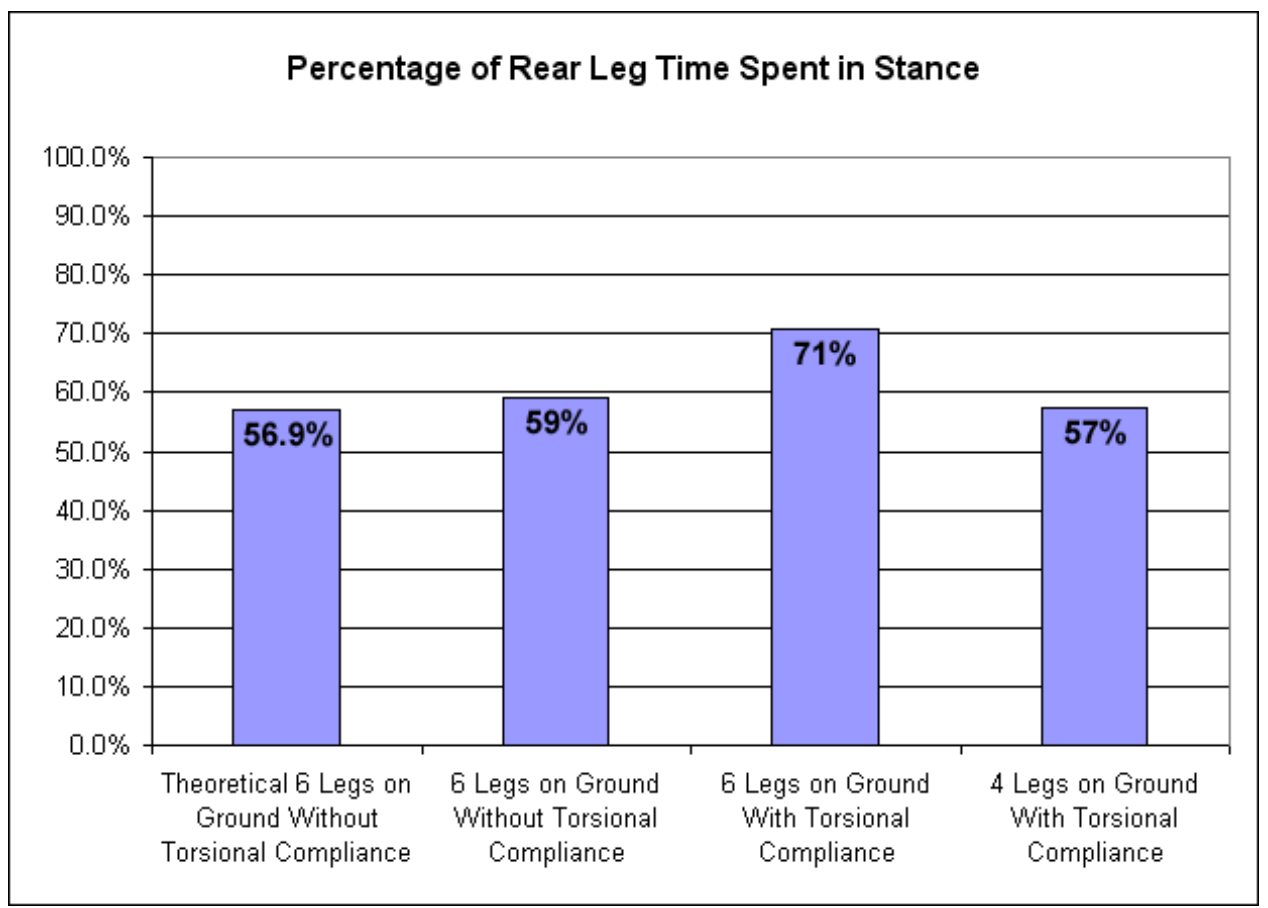

Figure 10-3. Percentage of Rear Leg Time Spent in Stance

Figure 10-3 shows the percentage of the time a rear leg spent in stance in each of the three tests, where stance was defined as any time during which the foot was touching the ground. The figure also shows the prediction of the Mechanism Analyzer program in the first column.

The program prediced the rear leg would be in stance $56.9 \%$ of each cycle if no torsional compliance was available. When the robot was tested without torsional compliance, the rear leg spent 59\% of each cycle in stance. When the passive torsionally compliant devices were allowed to function properly and the robot walked on 6 legs, the rear leg spent $71 \%$ of each cycle in stance. The torsionally compliant devices again allowed the rear legs to spend a higher percentage of their time in stance, by winding up at the beginning of stance and quickly unwinding during swing. Stance in the rear legs 
was not, however, extended as much as it was with the middle legs. The middle legs were supporting a higher percentage of the weight. As a result, the middle legs wound the springs in their torsionally compliant devices more than the rear legs did.

Finally, when the body flexion joint was used to raise the front legs off the ground, the rear legs spent $57 \%$ of each cycle in stance. Stance was shorter than during the test with 6 legs on the ground, because the front legs hanging in the air acted like a lever and reduced the amount of weight on the rear legs. At the same time, the load on the middle legs increased.

The robot could walk with its front legs off of the ground with the use of torsionally compliant devices because the amount of time each leg spent in stance was increased. This divides the load between more legs and keeps the robot stable.

When the torsionally compliant devices are disabled, preventing relative rotation to occur between the input and output shafts of each device, the robot is unable to walk with its front legs off of the ground. In this scenario, the robot is forced to walk in a diagonal gait, with each middle leg moving in phase with the rear leg on the other side of the robot. The middle legs cannot be lifted off the ground, because the majority of the weight is over those legs. Each middle leg pushes backward when it is in stance. And when each middle leg is supposed to be in swing, it remains on the ground, pushing forward. As a result, no forward progress can be made, and the robot twists back and forth in the coronal plane. 


\subsection{Leg Coordination and Turning}

The activation of the torsionally compliant devices during walking changes the leg coordination. The term "air-walking” is used to describe a robot that is suspended such that the legs are no longer touching the ground. An inaccurate assumption was made during the initial tests of MechaRoach II that if the robot air-walked in an alternating tripod gait, it would continue to do so when placed on the ground. Because the torsionally compliant devices on the middle legs are wound more than the front and rear legs during walking, the middle legs get out of phase with the other legs when the robot is placed on the ground. In order to achieve a tripod gait while walking on the ground, the initial leg coordination was changed. When air-walking, the middle and rear legs on the same side of the robot were placed nearly in phase. By analyzing the crank position during air-walking, it was determined that the left middle leg was 10 degrees ahead of the phase of the left rear leg. When placed on the ground, the left middle and rear legs move 180 degrees out of phase due to the torsionally compliant devices.

During the initial tests in which MechaRoach II was setup so that it would air-walk in a tripod gait, the middle and rear legs on the same side of the robot would end up almost being in phase while walking on the ground. This caused the middle legs to support an even larger proportion of the weight, and the rear legs to be dragged across the ground. Dragging the rear legs across the ground was not optimal, and it prevented the robot from being able to turn. When the body steering joints were rotated, the front and rear legs attempted to rotate the robot, but the robot continued to progress in whatever direction the middle legs were pointed in. 
When tests were performed with torsional compliance locked out, the leg coordination was returned to the state in which the robot would air-walk in a tripod gait. Figure 10-4 shows once cycle of the gait pattern for MechaRoach II with the passive torsionally compliant devices locked out. The data for the figure were obtained by studying video of the robot walking in a straight line on a horizontal surface. The camera used to obtain the video recorded at 30 frames/sec.

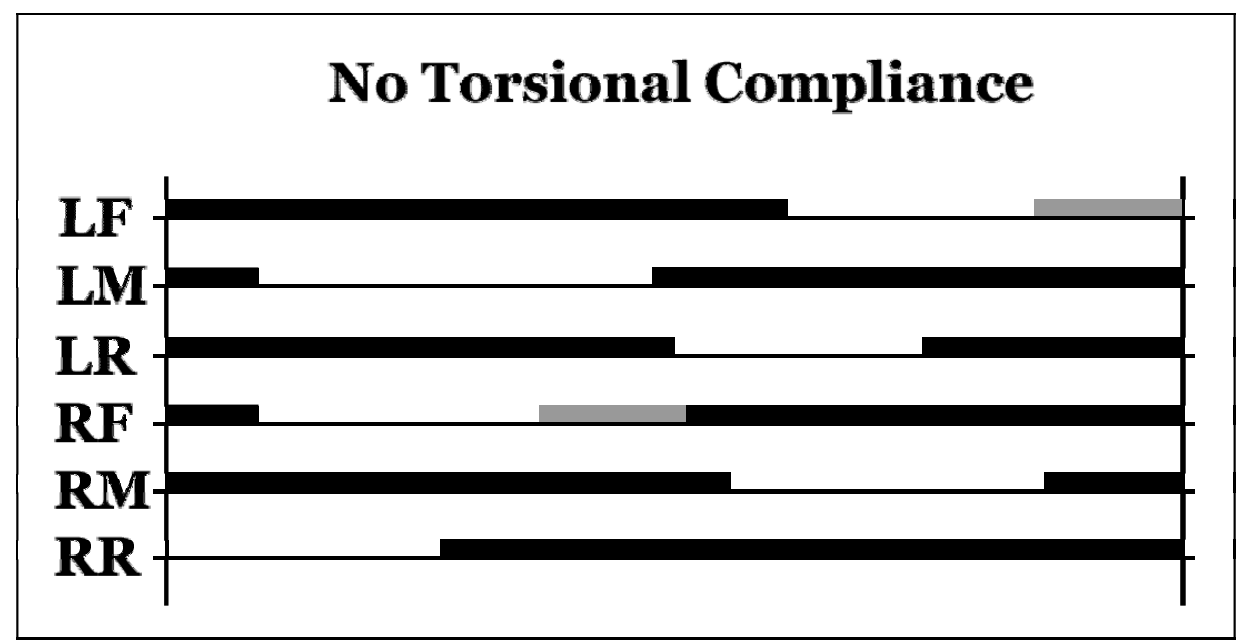

Figure 10-4. Gait Diagram for MechaRoach II with No Torsional compliance

The figure shows when the Left Front (LF), Left Middle (LM), Left Rear (LR), Right Front (RF), Right Middle (RM), and Right Rear (RR) legs are on the ground and when they are in the air during one complete walking cycle. The thick, black lines indicate when the leg was touching the ground. The thick, gray lines indicate when the front legs were in the air sweeping downward and could be in stance if an obstacle were in the way.

The legs were coordinated to walk in an alternating tripod gait. In a tripod gait, the front and rear legs on one side move in phase with the middle leg on the other side. The figure shows periods of time when only one tripod of support is on the ground and the other tripod is in swing. It also shows periods of overlap between the two tripods of support. 
Figure 10-5 shows one cycle of the gait pattern for MechaRoach II with torsional compliance.

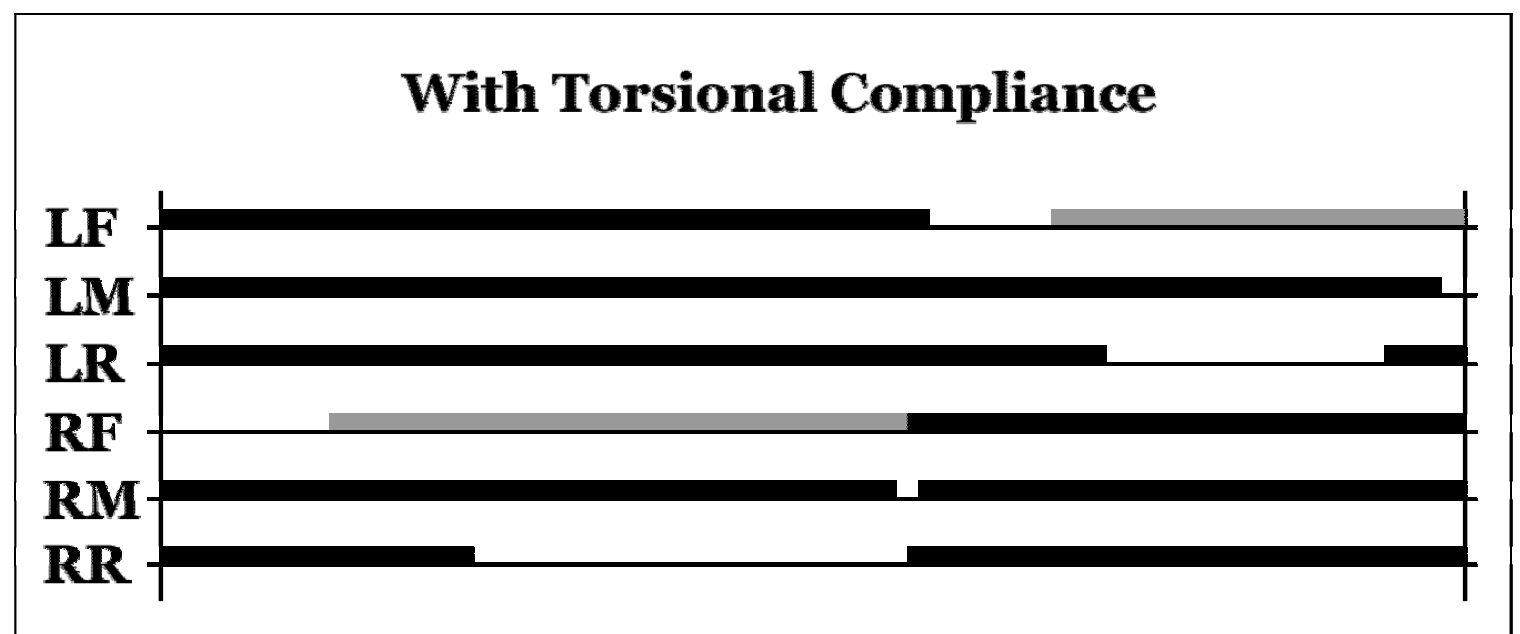

Figure 10-5. Gait Diagram for MechaRoach II with Torsional Compliance

Again, the thick black lines show when the corresponding legs are touching the ground. The thick, gray lines show when the front legs are sweeping downward and could be in stance if an obstacle were in the way.

When torsional compliance is allowed, there are almost no periods when more than two legs are off the ground at the same time. Having more legs on the ground at the same time stabilizes the robot by dividing the load between more legs.

The amount of time that the front legs spend in the air while sweeping downward is much longer, compared to when torsional compliance has been locked out. When the robot has no torsional compliance, front leg heel strike occurs much earlier in the trajectory, shortening the amount of time that the leg spends sweeping downward. Heel strike occurs earlier in the trajectory because of the increased amount of body roll that the chassis experiences. 
When the legs are coordinated properly, the robot is able to turn properly. MechaRoach II has a $60 \mathrm{~cm}$ turning radius on a horizontal surface. The turning radius is less than the $66 \mathrm{~cm}$ length of the robot.

If the claws had been fully implemented and had provided attachment forces while climbing steeply inclined surfaces, it was expected that leg coordination would be even more important for stability. If the legs were not operating in the proper gait, the robot would not have been supported at all times by at least three legs. Attachment forces generated by only two legs may not have been enough to support the robot on a steeply inclined surface, and torques may have been generated around the center of mass, causing unintended rotation of the robot in the coronal plane.

The use of driveshafts to transfer torque between the different sections of the robot allows the leg coordination to remain the same even when body joints are rotated. A tripod gait is maintained while turning, and when the body flexion joint has been activated. Leg coordination could not have been maintained if drivechains were used to transfer power between different sections of the robot, as explained in Section 9.3. The driveshafts require heavy components such as ball-splines and double universal joints to be used. In addition, the size of the ball-splines and double universal joints forces the front and rear legs to be farther from the middle legs than if drivechains had been used. Nevertheless, the results suggest that the use of driveshafts was the correct design choice. 


\subsection{Power Consumption}

MechaRoach II is power-autonomous. A single 4.8-volt, 600mAh rechargeable battery provides power for the radio-control receiver. A single 7.2-volt, 3000mAh NiCd rechargeable battery pack weighing 360g provides power for the motors.

During walking, approximately 3 amps are drawn from the main battery pack. A multimeter reading the current draw in series with one of the battery terminals showed that the current fluctuated between 2.5 and 3.5 amps, with the majority of the time spent in the middle of the range. The current draw was the same while air-walking. During climbing, the current draw was higher, and a maximum of just over 8 amps was recorded. A 10 A normal-blow fuse was installed in series with the main battery pack during construction, and the fuse was never blown during the operation of the robot.

When walking on a horizontal surface, MechaRoach II is able to operate for about 43 minutes before depleting the main battery pack. The time to depletion was measured while the robot was performing walking and turning tests. The robot was operated for a few minutes at a time, separated by short breaks to check for loose fasteners.

The battery pack that supplies the receiver with power lasts considerably longer, but tests were never performed to determine the operation time before depletion.

\subsection{Obstacle Climbing}

The obstacle climbing performance of MechaRoach II is limited by the reaching height of the middle and rear legs during swing. The robot is able to walk over a step up to $3.8 \mathrm{~cm}$ in height. When faced with a $5 \mathrm{~cm}$ step, the front and middle legs are able to step over it, but the rear legs are unable to. The body flexion joint can be used to extend 
the reaching height of the front legs, which helps them reach over steps. The body flexion joint can also be rotated downward in order to increase the reaching height of the middle legs. However, the body joint has no effect on the reaching height of the rear legs. A slightly higher performance may have resulted from redesigning the rear legs so that they have a higher reaching height during swing. However, during normal walking, the lowest part of the chassis lies about $4 \mathrm{~cm}$ above the ground. Therefore, even a redesign of the rear legs would probably not allow the robot to get over steps much higher than $3.8 \mathrm{~cm}$ due to insufficient ground clearance. This was of not much concern because the robot was specifically designed to have a center of gravity as close to the substrate as possible in order to aid in climbing inclined surfaces, and obstacle climbing performance was a secondary goal.

If the center of mass was not required to be as close to the substrate as possible, the legs could have been designed to be slightly longer, giving them higher reach during swing.

The distance in between leg pairs also negatively affects obstacle climbing. Had the front and rear legs been closer to the middle legs, the robot may have been able to climb higher steps. The leg pairs were required to be as far away as they were by the presence of the ball-splines and double universal joints. These components required a lot of space and could not be shortened. The use of drivechains would have allowed the leg pairs to be closer together, because the ball-splines and universal joints would not have been needed. 
The legs of the robot did not have any compliance in the longitudinal direction during swing. When a leg came into contact with an obstacle during swing, the entire robot was either pushed backwards, or the torque from the motor caused a drivechain failure.

MechaRoach I experiences the same problem, but it went unnoticed. The feet of MechaRoach I were made from strips of spring steel, which generate little traction. When a leg made contact with an obstacle during swing, the entire robot could easily be pushed backward due to the lack of traction.

Whegs ${ }^{\mathrm{TM}}$ robots also do not have compliance in the longitudinal direction during swing. The problem is less noticeable because their spokes move in a rotary manner, which raises the feet much higher in swing. Raising feet higher reduces the chance that a collision will occur during swing.

During normal walking, the driveshafts of MechaRoach II are located about $7 \mathrm{~cm}$ above the substrate. Therefore, an equivalently sized wheel or wheel-leg would have a $14 \mathrm{~cm}$ diameter. The robot could only climb up steps up to $3.8 \mathrm{~cm}$ in height, which is $27.1 \%$ of the diameter of the equivalently sized wheel or wheel-leg. The obstacle climbing performance of MechaRoach II is far less than that seen in Whegs ${ }^{\mathrm{TM}}$ robots, which can climb over obstacles up to $75 \%$ of their diameter, even without a body joint [3].

The obstacle climbing performance of MechaRoach II is probably less than that of the equivalently sized wheel. Wheeled vehicles certainly exist that can climb up steps higher than $27.1 \%$ of their diameter. However, due to insufficient ground clearance of the chassis, MechaRoach II would not likely be able to climb over higher obstacles if it had the equivalently sized wheels mounted to it. 


\subsection{Transitioning Between Horizontal and Inclined Surfaces}

MechaRoach II is able to make the transition between a horizontal surface and a surface that is inclined up to 20 degrees. With ramps inclined steeper than 20 degrees, the middle legs are unable to get into an efficient orientation, and the lack of traction prevents the robot from making forward progress.

Because Whegs ${ }^{\mathrm{TM}}$ move in a rotary manner, they are in the same orientation to the ground, no matter how the chassis is oriented in the sagittal plane. Therefore, when making the transition between a horizontal and inclined surface, they cannot be placed in an inefficient orientation with respect to the substrate.

MechaRoach II was tested on ramps that were covered with various surfaces. One ramp was covered in carpet, and another was coated in polyethylene liner that is typically used to line tool cabinet drawers. Both the carpet and the polyethylene liner seemed to allow the robot to receive the most traction.

Previous Whegs ${ }^{\mathrm{TM}}$ robots have used a coating of Devcon Flexane on their feet to give them traction. The resulting coating is rubber-like. The friction created between the rubber on the feet of MechaRoach II and the ramps it was operated on should have been at least as good as the interaction between the feet of the Whegs ${ }^{\mathrm{TM}}$ robots and standard floor surfaces. The feet of MechaRoach II could have been modified to provide a larger surface area and coated with Devcon Flexane. Performing this modification would have increased the traction that could be generated. However, other shortcomings of the robot would have prevented increased traction from significantly improving overall 
performance. For instance, ramps that are inclined more increase the chances that a swinging leg will collide with it during a transition to that surface.

A ramp was created with cleats to help the robot walk up steeper inclines. The cleats were $1.27 \mathrm{~cm}$ square in cross-section, and spaced $5 \mathrm{~cm}$ apart. These cleats were fastened to a ramp, and the ramp was inclined by various amounts. Unfortunately, the legs' lack of compliance in the longitudinal direction during swing prevented the robot from operating well on the cleated ramp. As with other kinds of obstacles, when the leg made contact with a cleat during swing, either the entire robot was pushed backward or the torque from the motor caused a drivetrain failure.

Transitions between horizontal and inclined surfaces were attempted without torsionally compliant devices. With the torsionally compliant devices locked out, the robot was able to negotiate the transition between a horizontal surface, and a surface inclined up to 20 degrees. The torsionally compliant devices, therefore, did not permit the robot to transition from horizontal surfaces to more sharply inclined surfaces.

\subsection{Body Flexion}

Tests were conducted in which transitions between horizontal and inclined surfaces were attempted without use of the body flexion joint. The body flexion joint was not back-drivable, and therefore would not move if not commanded to. The joint was left in a position where the front of the robot would be aligned with the rear section.

MechaRoach II was able to transition from a horizontal surface to a ramp inclined 10 degrees without using the body flexion joint. When the ramp was inclined farther, the middle legs were lifted off the substrate in the transition area. Figure 10-6 shows the 
robot attempting to transition from walking on a horizontal surface to an inclined one, without using the body flexion joint. The middle legs are lifted off of the ground in the transition area, leaving the robot to rely only on the front and rear legs for propulsion.

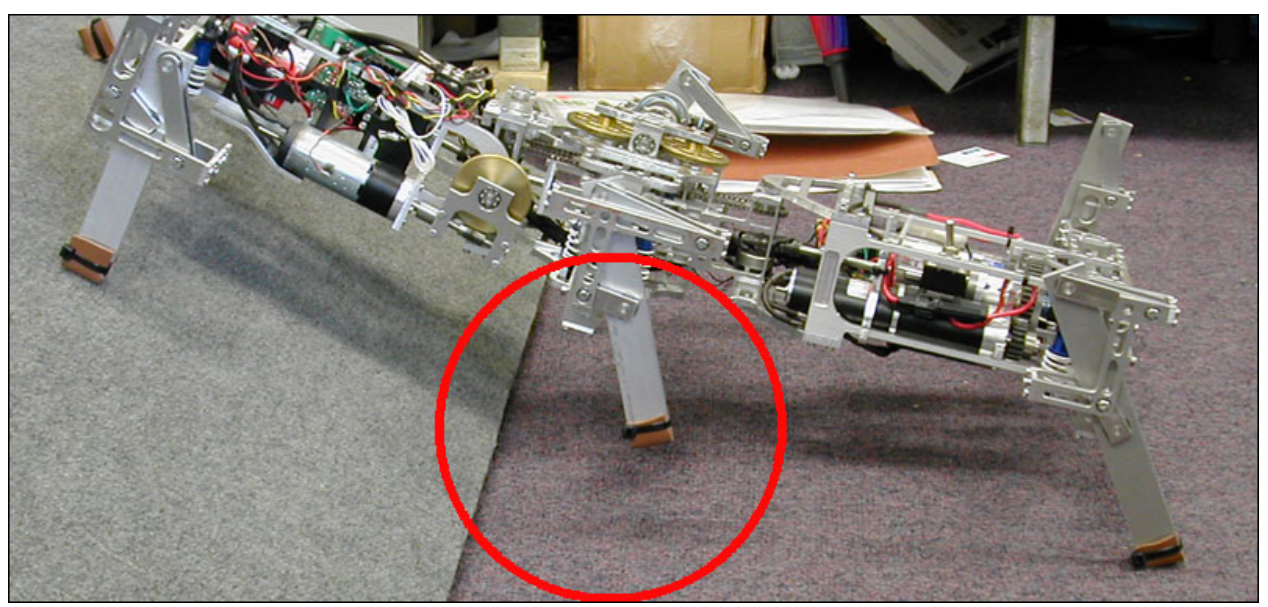

Figure 10-6. Transitioning without Use of Body Flexion Joint

Because the front legs begin stance by creating decelerating forces in the horizontal direction, they are unable to pull the robot forward in an effective manner. The body flexion joint allows the middle legs to continue to engage the substrate, and makes possible the transition to a steeper ramp. Figure 10-7 shows the robot attempting the same transition while using the body flexion joint. The use of the body flexion joint allows the middle legs to remain in contact with the substrate through the transition area.

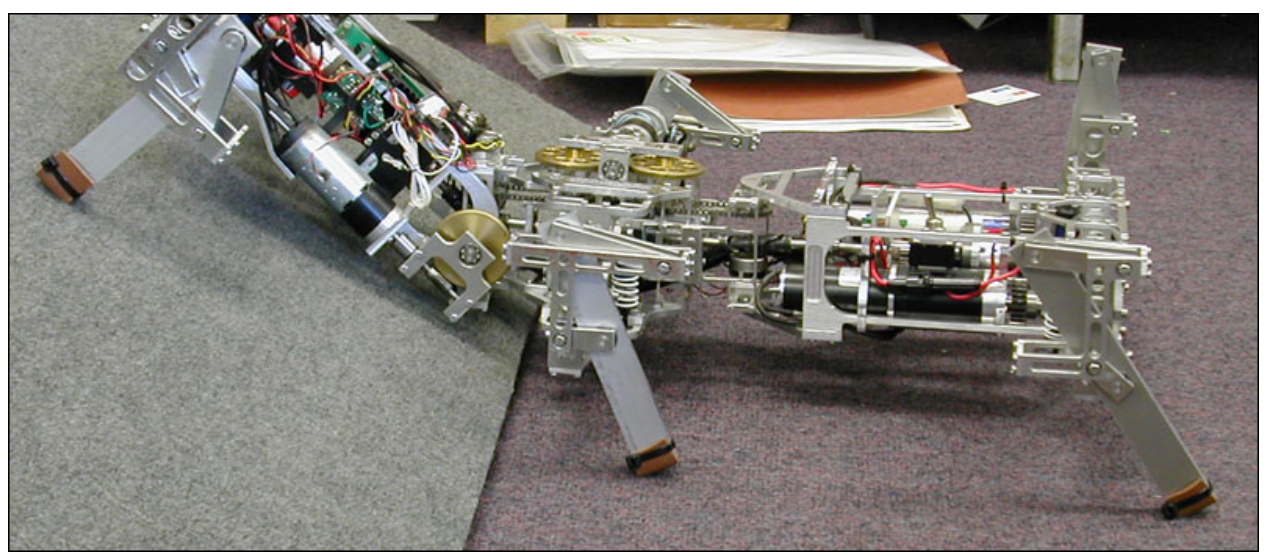

Figure 10-7. Transitioning with Use of Body Flexion Joint 


\section{Conclusions}

MechaRoach II is a six-legged robot that utilized four different abstracted cockroach locomotion mechanisms, including usage of features of leg morphology, leg compliance, body flexion, and gait adaptation. The primary purpose of the robot was to test strategies for transitioning between horizontal and inclined surfaces.

The robot is capable of walking at 1/18 body lengths/second. A 7.2-volt main battery pack supplies power to the three motors located on-board. The robot is able to operate for 43 minutes before depletion of the main battery pack. The time to depletion is virtually the same as that of Whegs II, when its drive motor is powered by a single 7.2volt battery pack [2]. Whegs II uses a similar drive motor to the one used in MechaRoach II, but its steering and body joints are powered by a separate battery pack.

Various techniques including graphical synthesis, numerical analysis and prototyping were used to design 4-bar mechanisms to be used as legs. The 4-bar mechanisms had a multitude of design constraints to satisfy, including reproducing features of cockroach footpaths that allow them to climb over relatively large obstacles without active changes in foot trajectory. These mechanisms allow the robot to climb over obstacles up to $3.8 \mathrm{~cm}$ high, but end up in inefficient positions when faced with transitioning onto steep inclines. The robot's obstacle climbing ability was a secondary goal, and was compromised by mechanical advantage considerations, insufficient ground clearance, and the reaching height of the rear leg during swing.

Passive torsionally compliant devices were used for gait adaptation. They allow leg pairs to be brought into phase to work together when climbing. These devices are also 
used during normal walking to extend the stance phase of each leg cycle, and to reduce the amount of time spent in swing.

The torsionally compliant devices allow 180 degrees of relative rotation between an input and an output shaft. Because the middle legs are loaded heavier than the front and rear legs, they routinely wind the springs of their torsionally compliant devices more than the ones in the front and rear legs. When the legs transition to swing, their springs are rapidly unwound. When the body flexion joint is used to raise the front legs of the robot off the ground, the release of the springs in the middle leg torsionally compliant devices occurs in one frame of video. The video camera recorded 30 frames/second. The springs in all of the torsionally compliant devices on MechaRoach II have the same spring rate. Springs with higher rates could have been used in the middle leg torsionally compliant devices, while springs with lower rates could have been used in the front and rear leg torsionally compliant devices. Using different spring rates could have resulted in all of the devices being wound the same amount while walking on a horizontal surface.

When the springs are released, loud clicking noises are heard from the robot. The energy being released when the springs are unwound causes shock to be transmitted to the rest of the chassis and drivetrain. It is entirely possible this shock contributes to the problem of fasteners working themselves loose. The majority of fasteners that would regularly loosen themselves are located in the middle section of the robot. The addition of torsional damping to the torsionally compliant devices may have helped this problem. A torsional damper would have slowed the rate at which the energy was being released from the torsion springs. At the same time, the addition of a damper would increase the weight and complexity of the torsionally compliant devices. 
Though the robot was designed for testing strategies for transitioning between horizontal and inclined surfaces, the robot's ability to transition is hampered by a number of problems.

The largest problem is the lack of longitudinal compliance in the legs during swing. As a result, if the leg collides with an obstacle during swing, the torque from the drive motor either causes the leg to push the entire robot backward or causes a mechanical failure in the drivetrain. The same problem was present in MechaRoach I and the Whegs ${ }^{\mathrm{TM}}$ series of robots, but went unnoticed or only rarely caused serious problems.

There are several ways the problem could be resolved. Compliance in the longitudinal direction could be added to each leg. Each leg could be connected to the chassis through a hinge joint that pivots along a vertical axis. This hinge would be kept in a nominal position by a very stiff spring. The spring would only be displaced if a large load were imposed on the leg in the proper direction. The purpose of the spring would be to allow just enough compliance to prevent damage to occur to the drivetrain. If the leg collided with an obstacle during swing, the foot would attempt to push the entire robot backward, causing the leg to displace the spring. Displacement of the spring would occur until the leg transitioned into stance, at which time, the foot would stop pushing the robot backward. The spring would then return to its nominal position as the foot is drawn backward during stance. The operator would then have the opportunity to attempt to reposition the leg by using the body joints before the leg collides with the obstacle again.

Another solution to the problem would be to give each leg the equivalent of the elevator reflex seen in locusts by Pearson [29]. When locust legs collide with an obstacle during swing, the reflex causes the leg to reverse direction, then raises the leg higher and 
proceeds to swing the leg forward again. An abstraction of the concept could be added to the robot by adding another degree of freedom to each leg, allowing the entire leg assembly to be raised. When an operator sees a leg collide with an obstacle in swing, the drivetrain could be put into reverse for a short period of time, the leg could then be raised higher, and then the drivetrain could be driven forward again. The added degree of freedom may allow higher obstacles to be surmounted. It also might help in transitioning to steep ramps.

Another solution to the problem might be developing a link in the 4-bar leg mechanism that can change its length. Ritzmann first suggested the idea as a way to dynamically change a leg's walking trajectory into a climbing one [36]. If the leg made contact with an obstacle during swing, the reconfigurable link would be put into action, allowing the swinging leg to be swung higher.

In actuality, designing a mechanism with this capability would be very difficult. The design of a 4-bar mechanism with 4 static links that satisfied all of the constraints was very time consuming. The leg mechanisms were the most time-consuming components to design in the entire robot. Adding another degree of complexity to the mechanisms would make the design process that much more difficult and time-consuming.

One of the primary difficulties with designing a 4-bar mechanism to be used as a leg is adjusting the ratio between time spent in stance and time spent in swing. The middle and rear legs of the robot barely spend more than $50 \%$ of their cycles in stance. It was only because of the inclusion of torsionally compliant devices that a significant overlap between the two tripods of support was able to occur. Leg mechanisms that attempt to 
create cockroach-like footpaths should probably not be implemented without torsional compliance, for this reason.

The constraints that prevent stance from being a higher proportion of each leg's cycle are mechanical advantage and the location of the ground pivots. Had the ground pivot locations been allowed to be below the ground, it would have been much easier to design a mechanism that dwells in stance and moves the leg much faster in swing, due to the peculiarities of 4-bar mechanisms.

The number of parameters that define a 4-bar mechanism might lead one to believe that a mechanism's performance could be maximized through the use of a genetic algorithm. The 4 link lengths and the 2 ground pivot locations were the primary geometric parameters that were adjusted during the redesign phases. Because the ground pivot locations were defined in a 2-dimensional space, each of those comprises 2 parameters. There are, therefore, 8 variables that could be adjusted which affect the output trajectory.

Unfortunately, the constraints that the leg mechanisms must satisfy would be very difficult to express in a fitness function that a genetic algorithm could use. While a proper fitness function could surely be written given enough time, the effort required would leave the use of a genetic algorithm outside the scope of this project.

Another major factor that hindered MechaRoach II's ability to climb is the front legs creating decelerating forces in the horizontal direction during stance. The front legs of the robot create decelerating forces because they were modeled after front legs of a cockroach, which create decelerating forces while walking. During walking and running, decelerating forces from the front legs may be a positive characteristic, but it is a 
hindrance during climbing. These forces prevented the robot from being able to make forward progress when just the front and rear legs could touch the ground. One possible solution would be to replace the front legs with mechanisms similar to the middle and rear legs. The front legs would have a reduced reaching height, but use of the body flexion joint reduces the need for front leg reaching height during swing. This solution could have been implemented, but probably would have made the probability of the leg colliding with an obstacle during swing higher, since it is the front legs that most often had this problem.

During a transition from a horizontal surface to a sharply inclined one, the middle legs frequently find themselves in inefficient orientations with respect to the ground. These orientations prevent the middle legs from propelling the robot forward, even though they are making contact with the ground. One possible solution would be to provide the robot with a total of two body flexion joints. One joint would allow the front section of the robot to be rotated upward and downward independently of the middle and rear sections. The other body flexion joint would allow the rear section to be rotated upward or downward independently of the middle and front sections. Proper coordination of the two body flexion joints could allow the middle legs to always assume an efficient orientation with respect to the ground during a transition from a horizontal surface to a sharply inclined one.

Other than for the purposes of vibration reduction, the leg compliance did not have any other effects on the performance of the robot. The leg compliance would have had a much larger effect on the performance of MechaRoach I or Whegs VP, which move at a much higher rate. Because those robots move much faster, the loads transmitted through 
the legs into the bodies of the robots are higher. MechaRoach II probably would have seen more benefits from leg compliance at much higher speeds.

Driveshafts were used to transfer torque between the different sections of the robot. The use of driveshafts required relatively large and heavy ball-splines and double universal joints to be used. Because of their size, these devices forced the front and rear legs to be farther away from the middle legs than optimal, which compromised obstacleclimbing performance. The driveshafts did, however, allow the leg coordination to remain the same even when body joints were rotated. The ability of the robot to maintain an alternating tripod gait even when turning or with the body joint rotated up or down was important for stability and for the ability to turn.

The abstracted cockroach locomotion mechanisms implemented on MechaRoach II have shown potential benefits for walking robots. However, the performance of the leg mechanisms has been shown to be too limited to be practical for a climbing robot. A single degree of freedom for each leg was not enough to allow MechaRoach II to negotiate a transition from a horizontal surface to a sharply inclined one. Additional body joints could be provided for the robot, or all of the legs could be designed to have additional degrees of freedom. Unfortunately, with additional degrees of freedom, the control problem then begins to approach that which is seen on robots modeled directly after cockroaches and other animals. Embedding part of the locomotion controller into the mechanics of the robot has a limited value if the control problem is going to be just as difficult.

Even with these limitations, the 4-bar mechanisms used for legs have potential. MechaRoach I has already shown that recreating cockroach-like footpaths on a robot 
results in cockroach-like mobility [7]. In addition, the 4-bar mechanisms allow the front, middle and rear legs to be optimized for different tasks. Leg specialization has been difficult to achieve with wheel-legs, because all of the legs are fixed in a rotary motion, and generally have to be the same diameter. 


\title{
Appendix A. The Mechanism Analyzer Program
}

\author{
\#include <stdio.h> \\ \#include <stalib.h> \\ \#include <math.h> \\ \#define PI 3.1415926535897932384626433832795028841972
}

//This program was written for the analysis of a theoretical //4-bar leg mechanism

//Link 1 (L1) refers to the crank of the mechanism. Link 2 (L2) //refers to the coupler (tibia) of the mechanism. Link 3 (L3) //refers to the rocker of the mechanism. O1 refers to the first //ground link, where the crank connects to the chassis. 02 //refers to the second ground link, where the rocker connects to //the chassis through a passive pin joint. P1 refers to the //passive joint between the crank and the coupler. P2 refers to //the passive joint between the rocker and coupler.

//All values are in English units, unless otherwise specified.

\section{void main (void)}

\{

//L1 is the length of the crank, L2 is the length of the //coupler (tibia) link (distance from P1 to the foot), L2U //is the distance from P1 to P2 along the coupler, and L3 //is the length of the rocker. All in inches.

double L1, L2, L2U, L3;

//Coordinate system is located at the driveshaft axis (O1), //with the positive-x direction being forward, and the //positive-y direction being upward. O2[2] stores the $\mathrm{x}$ //and y coordinates of the rocker mount point (where it //connects to the chassis)

double 02 [2];

//P102 is the distance between points P1 and 02 . P1 is where //the crank connects to the coupler, and 02 is where the //rocker connects to the chassis of the robot.

double P1O2;

//P1 is the location where the crank connects to the coupler //link. P2 is the location where the couple connects to //the rocker link. Each array stores the $\mathrm{x}$ and $\mathrm{y}$ position //of these points.

double P1 [2], P2 [2];

$/ / a, h$, and $Q$ are temp variables that are needed in order to //determine the location of P2. To determine exactly what //they are, refer to diagram Al. Q is a location, which is //why it is an array. $a$ and $h$ are lengths.

double $a, h, Q[2]$;

//temp is a variable that is used when calculating the sqrt of //a quantity. temp stores the sum of the squares, so that //the sqrt can be taken in the next step

double temp;

//alpha is the position on the crank (input). The position is //measured relative to when the crank is pointed straight //up from the driveshaft. In degrees. The positive-x //direction is forward. If that is to the right, then the //crank has to rotate *clockwise*.

double alpha, revalpha;

//alpharad stores alpha in radians double alpharad;

//LIslope and L3slope store the slopes of the corresponding //links. The slopes are needed to calculate the location //of the coupler's instant center. b3 is the y-intercept 
//of a line that is oriented along the rocker link.

double L1slope, L3slope, b3;

//IC02 is the instant center of link 2 (coupler) with respect //to the ground (link 0)

double IC02 [2];

//L2radius is the distance between ICO2 and $\mathrm{P} 1$, which is the //radius that the coupler instantaneously appears to //revolve about

double L2radius;

//w1 is the absolute angular velocity of link 1 (which is a //constant for the purposes of this analysis). w2 is the //angular velocity of link 2, with respect to $\mathrm{P} 1$.

double w1, w2;

//Pldot contains the absolute $\mathrm{x}$ and $\mathrm{y}$ velocities of the point //P1

double Pldot [2] ;

//r1, r2 and r3 are the vectors that define link 1 (crank), //link 2 (tibia) and link 3 (rocker), respectively. rfoot //is the vector that starts at P1 and extends to the foot //of the leg. The vector is used to determine the //instantaneous velocity of the foot.

double r1 [2], r2 [2], r3 [2], rfoot [2];

//Vfoot contains the components of the absolute velocity of the //foot, in $\mathrm{x}-\mathrm{y}$ coordinates

double Vfoot [3];

//Pfoot contains the coordinates of the absolute foot position, //in inches

double Pfoot [2]

//MA is the instantaneous Mechanical Advantage

double MA;

int counter.

//Initialize the file for output, and then open for writing

FILE *ofp;

ofp = fopen ("outfile", "w");

fprintflofp, "space alpha P1 [0] P1[1] P1O2 a h Q[0] Q[1] P2 [0]

$\mathrm{P} 2$ [1] r2 [0] r2 [1] rfoot [0] rfoot [1] Pfoot[0] Pfoot [1] Vfoot [0]

Vfoot [1] Vfoot [2] $M A \backslash \mathrm{n}^{\prime \prime)}$;

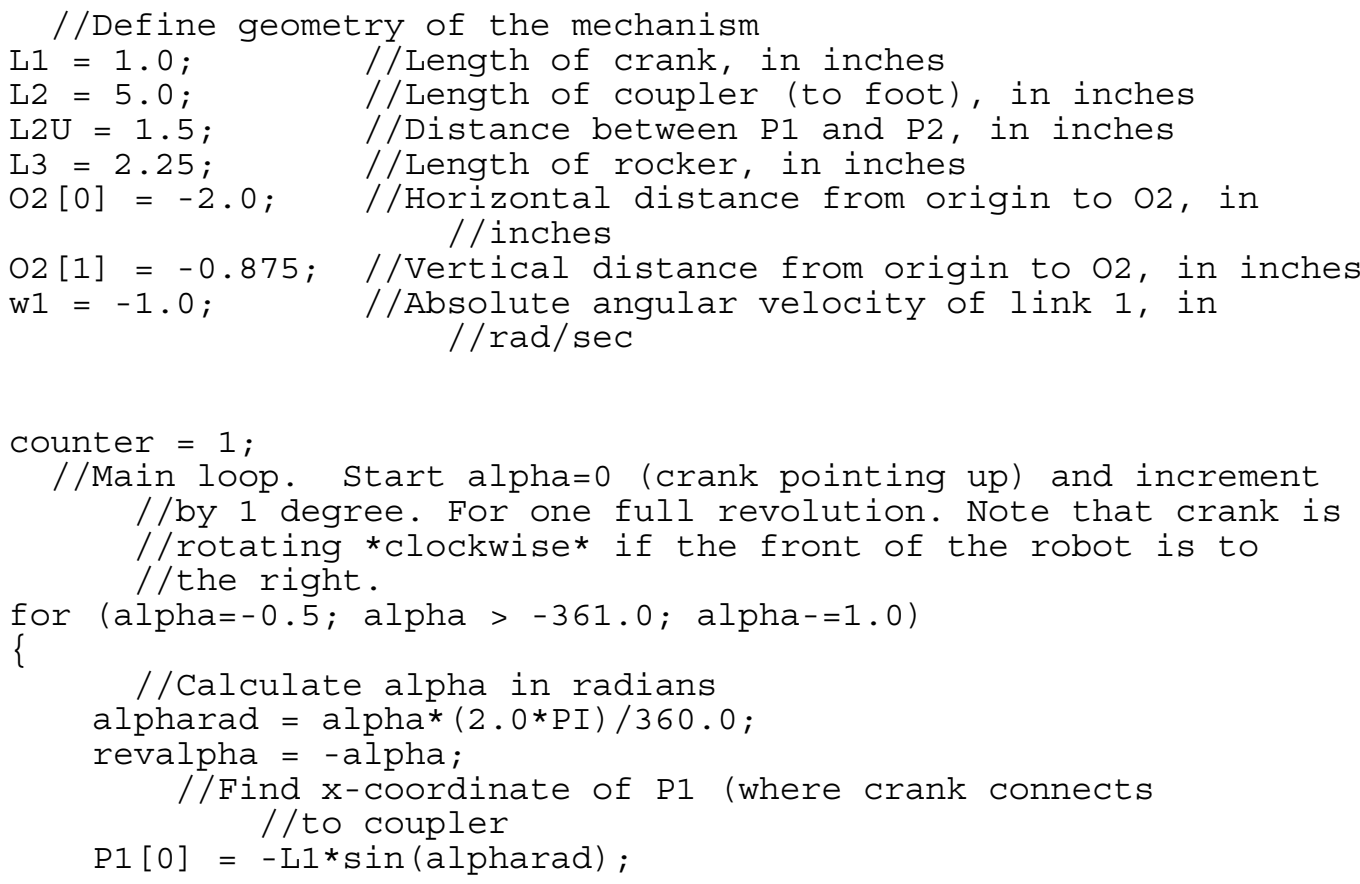




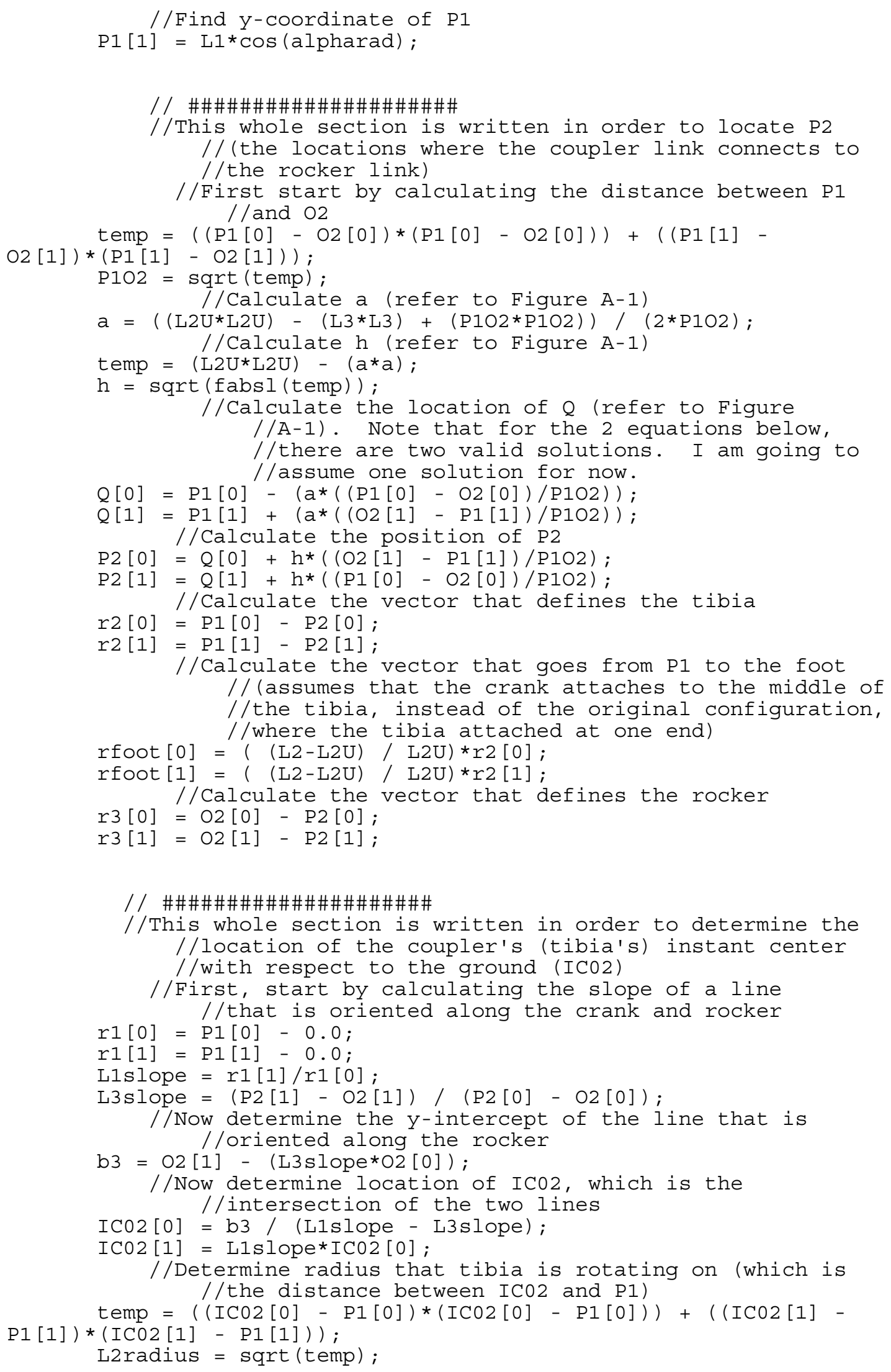




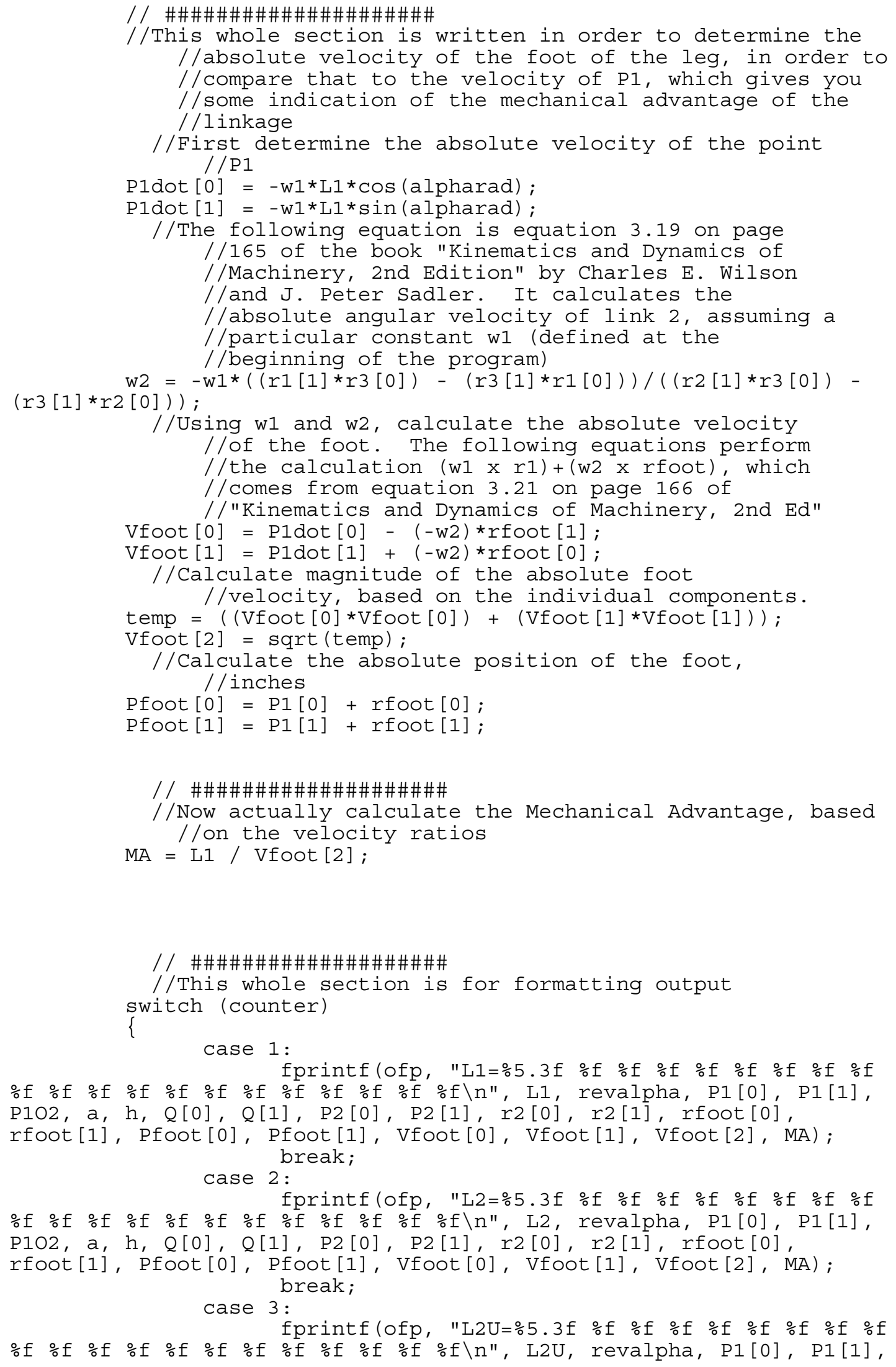




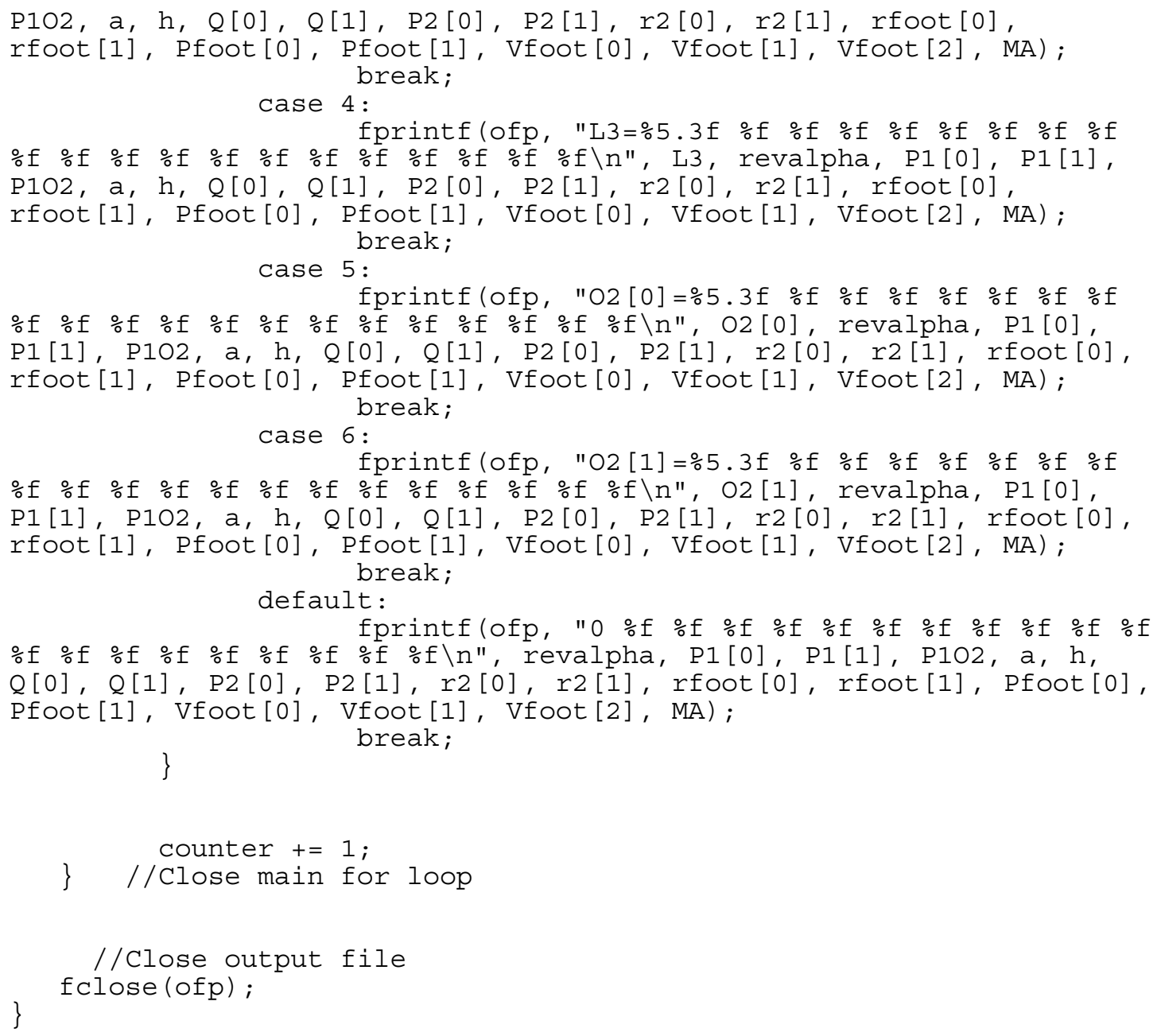




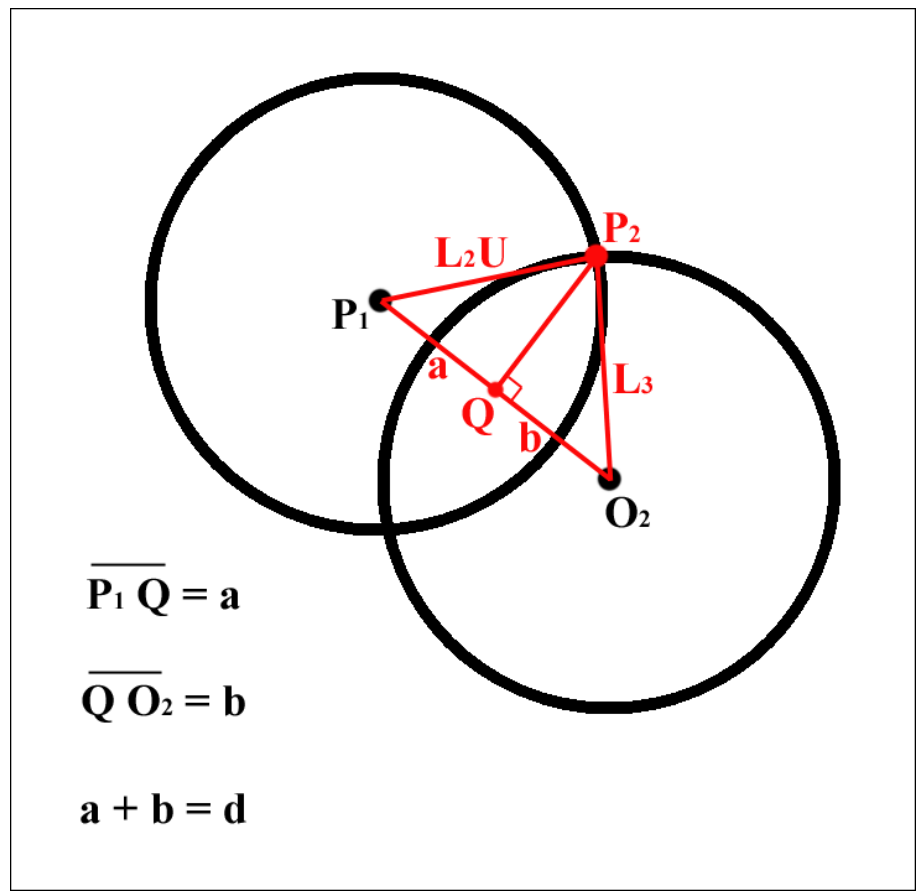

Figure A-1. Finding the Intersection of Two Circles 


\section{Bibliography}

1. Abderrahim, M, C. Balaguer, A. Gimenez, J.M. Pastor, and V.M. Padron (1999). ROMA: A Climbing Robot for Inspection Operations. Proceedings of the IEEE International Conference on Robotics and Automation, Volume 3, 10-15 May 1999.

2. Allen, T.J. (2004). Abstracted Biological Principles and Reduced Actuation Applied to the Design of a Walking Vehicle. Case Western Reserve University. M.S. Thesis.

3. Allen, T.J., R.D. Quinn, R.J. Bachmann, and R.E. Ritzmann (2003). Abstracted Biological Principles Applied with Reduced Actuation Improve Mobility of Legged Vehicles. IEEE International Conference On Intelligent Robots and Systems, Las Vegas, Nevada.

4. Autumn, K., W.-P. Chang, R. Fearing, T. Hsieh, T. Kenny, L. Liang, W. Zesch, and R.J. Full (2000). Adhesive Force of a Single Gecko Foot-Hair. Nature, 405, 681-685.

5. Autumn, K., M. Sitti, Y.A. Liang, A.M. Peattie, W.R. Hansen, S. Sponberg, T. Kenny, R. Fearing, J.N. Israelachvili, and R.J. Full (2002). Evidence For van der Waals Adhesion In Gecko Setae. Proceedings of the National Academy of Sciences, 99(19), 12252-12256.

6. Binnard, M (1995). Design of a Small Pneumatic Walking Robot. MIT. M.S. thesis.

7. Boggess, M.J., R.T. Schroer, R.D. Quinn, and R.E. Ritzmann (2004). Mechanized Cockroach Footpaths Enable Cockroach-like Mobility. Proceedings of IEEE 
International Conference on Robotics and Automation, New Orleans, LA, 26 April-1 May.

8. Collins, S.H., and Ruina, A. (2005) "A Bipedal Walking Robot with Efficient and Human-Like Gait," to appear in Proceedings of the IEEE International Conference on Robotics and Automation (ICRA '05), Barcelona.

9. Collins, S.H., Martijn Wisse and Andy Ruina (2001). A Three Dimensional Passive-Dynamic Walking Robot With 2 Legs and Knees. International Journal of Robotics Research, Vol 20, No 7, pp 607-615, July 2001.

10. Daltorio, K.A., A.D. Horchler, and R.D. Quinn (2005). A Small Wall-Walking Robot With Compliant, Adhesive Feet. In Press (IROS 2005).

11. Frazier, S. F., G.S. Larsen, D. Neff, L. Quimby, M. Carney, R.A. DiCaprio, and S.N. Zill (1999). Elasticity and movements of the cockroach tarsus in walking. Journal of Comparative Physiology, 185:157-172.

12. Full, R.J., R. Blickhan and L.H. Ting (1991). Leg Design in Hexapedal Runners. Journal of Experimental Biology, 158:369-390.

13. Galvez, J.A, P.G. de Santos, and F. Pfeiffer (2001). Intrinsic Tactile Sensing for the Optimization of Force Distribution in a Pipe Crawling Robot. IEEE/ASME Transactions on Mechatronics, Vol. 6, No. 1.

14. Guo, L., K. Rogers, and R. Kirkham (1994). A Climbing Robot With Continuous Motion. Proceedings of the IEEE International Conference on Robotics and Automation, 8-13 May, 1994. 
15. Hirose, S., and K. Kawabe (1998). Ceiling Walk of Quadruped Wall Climbing Robot. Proceedings of the First International Conference on Walking and Climbing Robots, Brussels.

16. Hirose, S, A. Nagakubo, and R. Toyama (1991). Machine That Can Walk and Climb on Floors, Walls and Ceilings. Proceedings of the IEEE International Conference on Robots in Advanced Robotics, 19-22 June 1991.

17. Janet, J., D. Reinfeld, and B. Wiedeman (2004). Vortex Regenerative Air Movement: Attraction and Attachment on Vertical and Inverted Surfaces: A Simple Method for Static and Mobile Robots for Climbing on Walls and Ceilings, U.S. Army Soldier and Biological Chemical Command, Soldier Systems Center, Natick, Massachusetts. NATICK/TR-04/013L, May 2004. DTIC\# ABV299170.

18. Jiao, Y., S. Gorb and M. Scherge (2000). Adhesion Measured on the Attachment Pads of Tettigonia viridissima (orthoptera, insecta). Journal of Experimental Biology, Vol 203: 1887-1895.

19. Jindrich, D.L and R.J. Full (2002). Dynamic Stabilization of Rapid Hexapedal Locomotion. Journal of Experimental Biology, Vol 205, 2803-2823.

20. Kim, S., A.T. Asbeck, M.R. Cutkosky, and W.R. Provancher (2005). SpinybotII: Climbing Hard Walls with Compliant Microspines. Proceedings of the IEEE International Conference on Advanced Robotics. July 18-20, 2005. Seattle, Washington.

21. Kram, R., B. Wong, and R.J. Full (1997). Three-Dimensional Kinematics and Limb Kinetic Energy of Running Cockroaches. Journal of Experimental Biology 200, 1919-1929. 
22. Lal Tummala, R., R. Mukherjee, N. Xi, D. Aslam, H. Dulimarta, J. Xiao, M. Minor, and G. Dang (2002). Climbing The Walls. IEEE Robotics and Automation Magazine, Vol 9, Issue 4, pp 10-19, December 2002.

23. Luk, B.L., D.S. Cooke, A.A. Collie, N.D. Hewer, and S. Chen (2001). Intelligent Legged Climbing Service Robot for Remote Inspection And Maintenance in Hazardous Environments. Proceedings of the IEEE Conference on Mechatrinics and Machine Vision in Practice, Hong Kong.

24. Luk, B.L., A.A. Collie, V. Piefort, and G.S. Virk (1996). Robug III: A Teleoperated Climbing and Walking Robot. UKACC International Conference on Control, Volume 1, Number 427.

25. Martin-Alvarez, A., W. de Peuter, J. Hillebrand, P. Putz, A. Mattheyssen, and J.F. de Weerd (1996). Walking Robots for Planetary Exploration Missions. Second World Automation Congress (WAC '96). May 27-30, 1996. Montpellier, France.

26. McGeer, T. (1990). Passive Dynamic Walking. International Journal of Robotics Research. 9:62-82.

27. Miyake, T, and H. Ishihara (2003). Mechanisms and Basic Properties of Window Cleaning Robot. Proceedings of the 2003 IEEE International Conference on Advanced Intelligent Mechatronics, Kobe, Japan, July 20-24, 2003.

28. Nishi, A (1991). A Wall Climbing Robot Using Propulsive Force of Propeller. Proceedings of the IEEE International Conference on Advanced Robotics, 19-22 June, 1991. 
29. Pearson, K.G. and R. Franklin (1984). Characteristics of Leg Movements and Patterns of Coordination in Locusts Walking on Rough Terrain. International Journal of Robotics Research 3(2), 101-112.

30. Pollack, A.J., R.E. Ritzmann, R.D. Quinn, and T.E. Wei (2002). Role of Higher Centers of the Insect CNS in Climbing and Incline Running. Society For Neurosciences, 2002 Annual Meeting, Orlando, FL.

31. Pollack, A.J., R.E. Ritzmann, A.L. Ridgel, and J. Archinal (2003). Incline Climbing Behaviors in Cockroach Require Intact Connections From Brain. Society For Neurosciences, 2003 Annual Meeting, New Orleans, LA.

32. Prahl, J.M. (2002). Personal Communication.

33. Quinn, R.D., G.M. Nelson, R.E. Ritzmann, R.J. Bachmann, D.A. Kingsley, J.T. Offi and T.J. Allen (2003). Parallel Strategies For Implementing Biological Principles Into Mobile Robots. International Journal of Robotics Research, Vol. 22 (3) pp. 169-186.

34. Ritzmann, R.E., A.J. Pollack, A.L. Ridgel, J. Archinal, and R.D. Quinn (2005). Descending Control of Body Attitude in the Cockroach Blaberus discoidalis And Its Role in Incline Climbing. Journal of Comparative Physiology A: Sensory, Neural, and Behavioral Physiology, Volume 191, Number 3, pp. 253-264.

35. Rizmann, R.E., R.D. Quinn, and M.S. Fischer (2004). Convergent Evolution and Locomotion Through Complex Terrain by Insects. To appear in Arthropod Structure and Development, Special Issue on Arthropod Locomotion and Biorobotics.

36. Ritzmann, R.E. (2005). Personal Communication. 
37. Roeder, K.D. (1948). Organization of the Ascending Giant Fibre System in the Cockroach, Periplaneta americana. J. Exp. Zool. 108: 243-261.

38. Saranli, U., M. Buehler, and D. Koditschek (2001). RHex: A Simple and Highly Mobile Hexapod Robot. International Journal of Robotics Research, 20(7): 616631.

39. Sandoor, G.N. and Arthur G. Erdman. Advanced Mechanism Design: Analysis and Synthesis, Vol. 2. Prentice Hall, Inc: Englewood Cliffs, NJ. 1984.

40. Saunders, Fenella (2000). Robo-Gecko - Gecko Robots. Discover, Sept 2000.

41. Schroer, R.T., Boggess, M.J., Bachmann, R.J., Quinn, R.D., and Ritzmann, R.E. (2004) "Comparing Cockroach and Whegs Robot Body Motions," IEEE Conference on Robotics and Automation (ICRA '04), New Orleans.

42. Sitti, M and R.S. Fearing (2003). Synthetic Gecko Foot-Hair Micro/NanoStructures for Future Wall-Climbing Robots. Proceedings of the 2003 IEEE International Conference on Robotics and Automation, Taipei, Taiwan, September 14-19.

43. Ting, L.H., R. Blickhan, and R.J. Full (1994). Dynamic and Static Stability in Hexapedal Runners. Journal of Experimental Biology, Vol. 197 : 251-269.

44. Watson, J.T., R.E. Ritzmann, S.N. Zill, A.J. Pollack (2002). Control of Obstacle Climbing in the Cockroach, Blaberus discoidalis: I. Kinematics. Journal of Comparative Physiology, Vol. 188: 39-53.

45. White, T.S., N. Hewer, B.L. Luk, and J. Hazel (1998). The Design and Operational Performance of a Climbing Robot Used for Weld Inspection in 
Hazardous Environments. Proceedings of the IEEE International Conference on Control Applications, Trieste, Italy.

46. Wilson, C.E. and J.P. Sadler. Kinematics and Dynamics of Machinery, 2nd Ed. New York: Harper Collins, 1993.

47. Wilson, D. M. (1966). Insect walking. A. Rev. Entom. 11: 103-123.

48. Yano, T., T. Suwa, M. Murakami, and T. Yamamoto (1997). Development of a Semi Self-Contained Wall Climbing Robot with Scanning Type Suction Cups. Proceedings of the IEEE International Conference on Intelligent Robots and Systems, Grenoble, France, 7-11 September, 1997. 